\title{
Amine-boranes as dual-purpose reagents for direct amidation of carboxylic acids
}

\author{
P. Veeraraghavan Ramachandran, * Henry J. Hamann, and Shivani Choudhary \\ $\dagger$ Herbert C. Brown Center for Borane Research, Department of Chemistry, Purdue University, \\ West Lafayette, Indiana 47907, United States \\ E-mail: chandran@purdue.edu
}

\section{Contents:}

General information S2

Preparation of ammonia-borane. $\mathrm{S} 2$

Preparation of amine-boranes via salt metathesis

Preparation of amine-boranes via sodium bicarbonate

Preparation of aniline borane via Lewis-base exchange $\mathrm{S} 4$

Optimization of amide synthesis.

Preparation of amides S5-S6

Procedures for mechanistic studies S6-S8

Crystallographic information for 4 aa. S9

Characterization of ammonia-borane and amine-boranes S10-S14

Characterization of amides S15-S26

Characterization of mechanistic intermediates S26-S27

References S28

NMR spectra of ammonia-borane and amine-boranes S29-S58

NMR spectra of amide products S59-S100

NMR spectra for mechanistic studies S100-S104 


\section{Experimental Section}

\section{General Information:}

Unless otherwise noted, all manipulations were carried out under open air conditions. ${ }^{11} \mathrm{~B},{ }^{19} \mathrm{~F},{ }^{13} \mathrm{C}$, and ${ }^{1} \mathrm{H}$ NMR spectra were recorded at room temperature, on a Varian INOVA $300 \mathrm{MHz}$ NMR spectrophotometer. Chemical shifts ( $\delta$ values) are reported in parts per million relative to $\mathrm{BF}_{3}$. $\mathrm{Et}_{2} \mathrm{O}$ for ${ }^{11} \mathrm{~B}$ NMR. Data are reported as: $\delta$ value, multiplicity $(\mathrm{s}=$ singlet, $\mathrm{d}=$ doublet, $\mathrm{t}=$ triplet, $\mathrm{q}=$ quartet, $\mathrm{p}=$ pentet, $\mathrm{h}=$ hextet, $\mathrm{m}=$ multiplet, $\mathrm{br}=$ broad) and integration. All solvents for routine isolation of products were reagent-grade. Sodium borohydride (powder, purity $>99 \%$ by hydride estimation ${ }^{1}$ ) was purchased in bulk from Dow Chemical Co. (Rohm and Haas). Ammonium sulfate (ACS reagent $>99.0 \%$, Fisher Chemical), was powdered prior to use. Tetrahydrofuran (THF, ACS reagent $>99.0 \%$ containing $0.004 \%$ water and $0.025 \%$ BHT) was purchased from Sigma-Aldrich. All amines and carboxylic acids were purchased from commercial sources and used without further purification.

\section{Preparation of ammonia-borane ${ }^{2}$ (procedure i-a):}

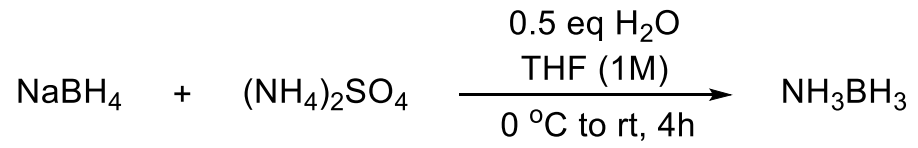

Scheme S1. Reaction equation for the preparation of ammonia-borane.

Sodium borohydride (18.91 g, $500 \mathrm{mmol}, 1$ eq.) and powdered ammonia sulfate $(66.07 \mathrm{~g}, 500$ mmol, 1 eq.) were transferred to a dry $2 \mathrm{~L}$ round bottom flask containing a large magnetic stir bar. The flask was then cooled in an ice-water bath followed by the addition of $495 \mathrm{~mL}$ of reagentgrade tetrahydrofuran. With vigorous stirring $4.5 \mathrm{~mL}$ of water was then added dropwise over a period of 5 minutes to limit frothing. Once all water is added the flask is moved to room temperature and stirred vigorously. The reaction is monitored using ${ }^{11} \mathrm{~B}$ NMR until completion $(\sim 4 \mathrm{~h})$ as judged by the absence of sodium borohydride peaks in the ${ }^{11} \mathrm{~B}$ NMR. (Prior to running the ${ }^{11} \mathrm{~B}$ NMR experiments, a drop of dimethyl sulfoxide is added to the aliquot to solubilize any sodium borohydride present.) Once complete the reaction mixture is filtered through celite and the filter cake thoroughly rinsed with tetrahydrofuran. The combined filtrates are condensed via rotary evaporation following by drying in vасио for $12 \mathrm{~h}$.

\section{Preparation of amine boranes via salt metathesis ${ }^{3}$ (procedure $\left.\mathrm{i}-\mathrm{b}\right)$ :}

$$
\begin{aligned}
& \mathrm{NaBH}_{4}+\underset{\mathrm{R}^{\prime \prime}}{\stackrel{\mathrm{NH}^{\prime}}{\oplus} \mathrm{H}_{2} \mathrm{X}^{\ominus}} \stackrel{\mathrm{THF}(1 \mathrm{M})}{\mathrm{rt}, 1-24 \mathrm{~h}} \underset{\mathrm{R}^{\prime \prime}}{\stackrel{\mathrm{RN}}{\mathrm{R}} \rightarrow \mathrm{BH}_{3}} \\
& X=\mathrm{Cl}, \mathrm{HSO}_{4}
\end{aligned}
$$

Scheme S2. Reaction equation for the preparation of amine-boranes from ammonium salts. 
Sodium borohydride $(0.76 \mathrm{~g}, 20 \mathrm{mmol})$ and the appropriate ammonium salt $(20 \mathrm{mmol})$ were transferred to a $100 \mathrm{~mL}$ dry round bottom flask, charged with a magnetic stir-bar. This was followed by addition of reagent-grade tetrahydrofuran $(20.0 \mathrm{~mL})$ at $\mathrm{rt}$. Reaction progress was monitored by 11B NMR spectroscopy (Note: A drop of anhydrous DMSO is added to the reaction aliquot before running the 11B NMR experiment to solubilized $\mathrm{NaBH}_{4}$ ). Upon completion of the reaction (1-24 h, as determined by 11B NMR), the reaction contents were filtered through sodium sulfate and celite and the solid residue washed with THF. Removal of the solvent in vacuo from the filtrate yielded the corresponding amine-borane. No further purification was necessary in the examples (1b-d, $\mathbf{p}, \mathbf{r})$ presented here.

\section{Preparation of amine boranes via sodium bicarbonate ${ }^{4}$ (procedure ii):}

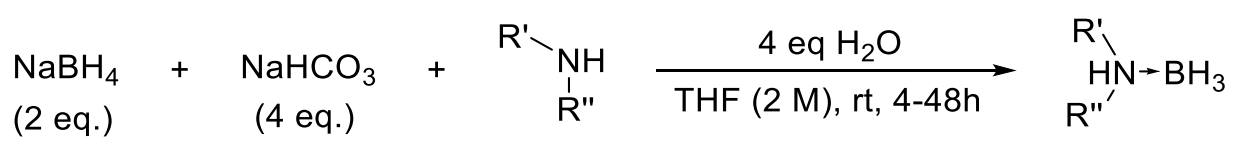

Scheme S3. Reaction equation for the preparation of amine-boranes from free amines.

Sodium borohydride (1.51 g, 2 eq., $40 \mathrm{mmol}$ ) and powdered sodium bicarbonate $(6.72 \mathrm{~g}, 4$ eq., 80 $\mathrm{mmol}$ ) were transferred to a $100 \mathrm{~mL}$ dry round bottom flask, charged with a magnetic stir-bar. The corresponding amine (1 eq., $20 \mathrm{mmol}$ ) was charged into the reaction flask followed by addition of reagent-grade tetrahydrofuran $(20 \mathrm{~mL})$ at $\mathrm{rt}$. Under vigorous stirring, water $(0.36 \mathrm{~mL}, 4$ eq., 80 mmol) was added drop-wise to prevent excessive frothing. Reaction progress was monitored by ${ }^{11} \mathrm{~B}$ NMR spectroscopy (Note: A drop of anhydrous DMSO is added to the reaction aliquot before running the ${ }^{11} \mathrm{~B} \mathrm{NMR}$ experiment to solubilize $\mathrm{NaBH}_{4}$ ). Upon completion of the reaction (4-48 $\mathrm{h}$, as determined by $\left.{ }^{11} \mathrm{~B} \mathrm{NMR}\right)$, the reaction contents were filtered through sodium sulfate and celite and the solid residue washed with THF. Removal of the solvent in vacuo from the filtrate yielded the corresponding amine-borane (1e-o, $\mathbf{q}, \mathbf{s})$. Residual solvent was removed by placing under highvacuum for $\sim 12 \mathrm{~h}$. In the case of valinol-borane (1s) additional work-up, detailed below, was necessary to break-up the partial formation of an intermediate amine-borane borate.

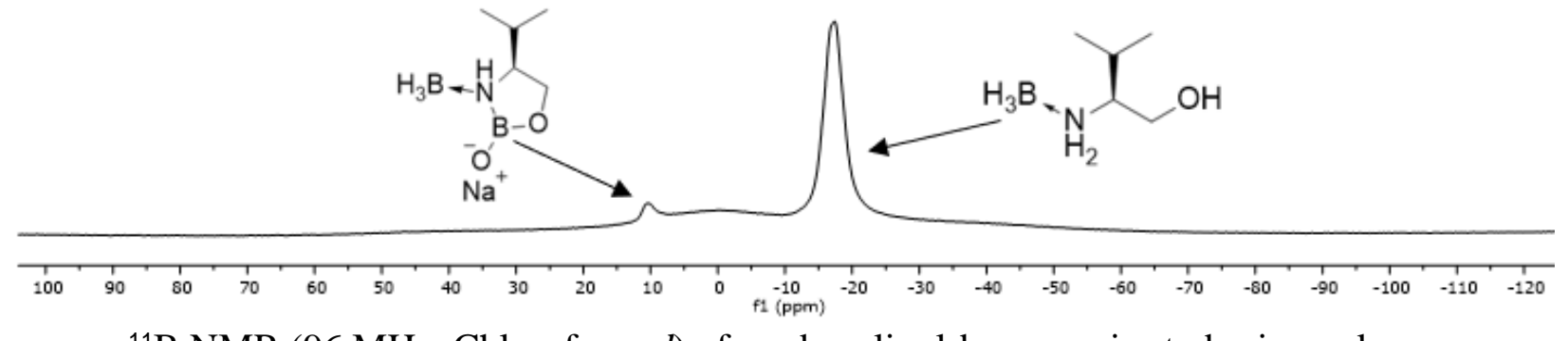

${ }^{11} \mathrm{~B}$ NMR (96 MHz, Chloroform- $d$ ) of crude valinol-borane prior to basic work-up.

Valinol-borane work-up: The crude amine-borane material obtained after celite filtration was placed in a $100 \mathrm{~mL}$ round bottom flask and dissolved was diethyl ether $(20 \mathrm{~mL})$. The solution was brought to $0{ }^{\circ} \mathrm{C}$. Then a dilute $\mathrm{NaOH}$ solution $(20 \mathrm{~mL}, 0.05 \mathrm{M})$ was added slowly to the amineborane solution. The mixture was moved to rt and stirred vigorously for 3 hours. After completion 
the volatiles were removed via rotary evaporation, and the remaining aqueous fraction was extracted with chloroform ( $3 \times 50 \mathrm{~mL}$ ). The organic extracts were dried over $\mathrm{MgSO}_{4}$, filtered through cotton and condensed via rotary evaporation. Residual solvent was removed by placing under high-vacuum for $\sim 12 \mathrm{~h}$.

\section{Preparation of aniline borane via Lewis-base $\operatorname{exchange}^{5}$ (procedure iii):}

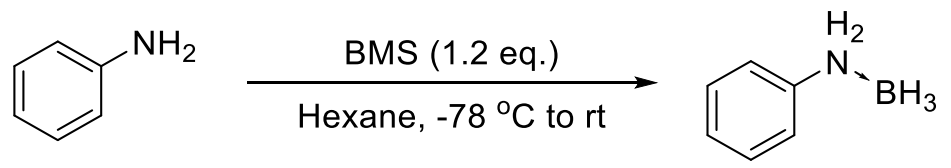

Scheme S4. Reaction equation for the preparation of aniline-borane from aniline and BMS.

Anhydrous hexanes ( $25 \mathrm{~mL}, 1 \mathrm{M}$ w.r.t. amine) was transferred to a $100 \mathrm{~mL}$ dry round bottom flask, charged with a magnetic stir-bar. The flask was sealed with a rubber septum and boranedimethylsulfide (BMS) $(3.3 \mathrm{~mL}, 1.2 \mathrm{eq} ., 30 \mathrm{mmol})$ was added via syringe at rt. After BMS addition the solution was brought to $-78^{\circ} \mathrm{C}$. Then at $-78{ }^{\circ} \mathrm{C}$ aniline $(2.3 \mathrm{~mL}, 1$ eq., $25 \mathrm{mmol})$ was added via syringe. The frozen mixture was stirred and gradually brought to rt whereupon a white precipitate formed. The mixture was stirred at $\mathrm{rt}$ for 1 hour, then the solvent was removed using a cotton plugged plastic pipette. The white solid was washed with hexanes $(6 \mathrm{x} 10 \mathrm{~mL})$ each time removing the solvent using a cotton plugged plastic pipette. Residual solvent was removed by placing under high-vacuum for $\sim 12 \mathrm{~h}$.

\section{Optimization of amide synthesis:}

\section{Optimization of Reagent Equivalents}

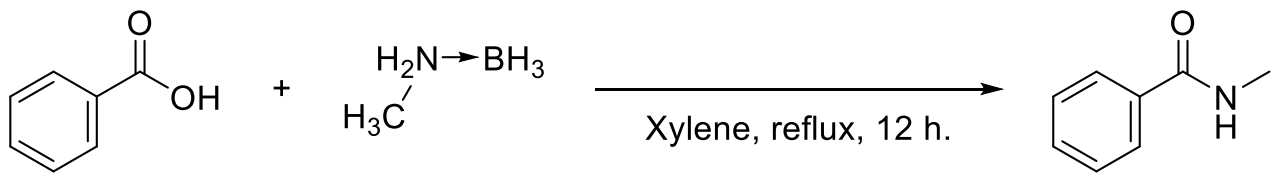

Scheme S5. Reaction equation for the study of required acid equivalents.

In a $25 \mathrm{ml}$ round bottom flask containing a magnetic stir bar was weighed methylamine-borane ( $0.090 \mathrm{~g}, 2 \mathrm{mmol}, 1$ eq.) followed by benzoic acid (1, 2, 3, or $4 \mathrm{eq})$. Xylenes was then added to the flask, (1 $\mathrm{M}$ with respect to benzoic acid). A reflux condenser was attached to the flask and the reaction mixture was brought to reflux. After 12 hours the reaction mixture was diluted with methanol $(50 \mathrm{~mL})$ and condensed via rotary evaporation. The crude mixture was diluted with ethyl acetate $(15 \mathrm{~mL})$ and transferred to a separatory funnel. The mixture was washed with cold $3 \mathrm{M}$ sodium hydroxide solution $(3 \times 10 \mathrm{~mL})$, then washed with brine $(1 \times 15 \mathrm{~mL})$. The organic layer was dried with sodium sulfate, filtered through cotton, and condensed via rotary evaporation. Residual solvent was removed by placing under high-vacuum for $\sim 12 \mathrm{~h}$. Results of the study are summarized below in Table $\mathrm{S} 1$. 


\begin{tabular}{|c|c|c|}
\hline Benzoic Acid & Methylamine-Borane & N-Methyl Benzamide Yield \\
\hline 1 eq. & 1 eq. & $49 \%(12 \mathrm{~h})$ \\
\hline 2eq. & 1 eq. & $78 \%(12 \mathrm{~h})$ \\
\hline 3 eq. & $\mathbf{1 ~ e q . ~}$ & $\mathbf{9 0 \%}(\mathbf{1 2} \mathbf{~ h})$ \\
\hline 4 eq. & 1 eq. & $89 \%(12 \mathrm{~h})$ \\
\hline
\end{tabular}

Table S1. Summary of results of the reagent equivalency study.

\section{Optimization of Reaction Conditions}

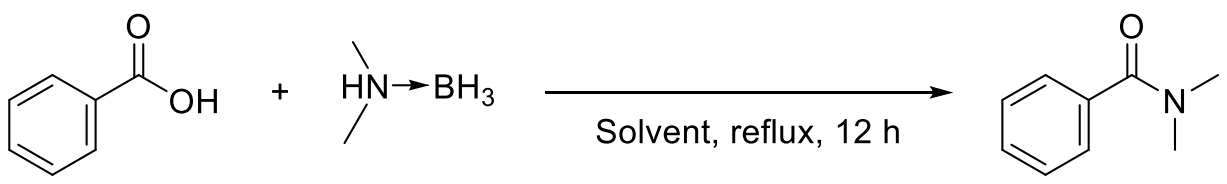

Scheme S6. Reaction equation for the study of optimal reaction conditions.

In a $25 \mathrm{ml}$ round bottom flask containing a magnetic stir bar was weighed dimethylamine-borane (0.118 g, 2 mmol, 1 eq.) followed by benzoic acid (0.733 g, 6 mmol, 3 eq.). The tested solvent was then added to the flask, $(6 \mathrm{~mL}, 1 \mathrm{M}$ with respect to benzoic acid). A reflux condenser was attached to the flask and the reaction mixture was brought to reflux. After 12 hours the reaction mixture was condensed via rotary evaporation (diluted with methanol $(50 \mathrm{~mL})$ in the reactions utilizing xylenes and toluene). The crude mixture was diluted with ethyl acetate $(15 \mathrm{~mL})$ and transferred to a separatory funnel. The mixture was washed with cold 3M sodium hydroxide solution $(3 \times 10 \mathrm{~mL})$, then washed with brine $(1 \times 15 \mathrm{~mL})$. The organic layer was dried with sodium sulfate, filtered through cotton, and condensed via rotary evaporation. Residual solvent was removed by placing under high-vacuum for $\sim 12 \mathrm{~h}$. Results of the study are summarized below in Table S2.

\begin{tabular}{|c|c|c|c|}
\hline Solvent (reflux) & Benzoic Acid & (Dimethylamine-Borane & Yield \\
\hline Xylenes & $\mathbf{3}$ eq. & $\mathbf{1}$ eq. & $\mathbf{9 9 \%}$ \\
\hline Xylenes +3 eq. $\mathrm{H}_{2} \mathrm{O}$ & 3 eq. & 1 eq. & $89 \%$ \\
\hline Toluene & 3 eq. & 1 eq. & $27 \%$ \\
\hline Toluene +3 eq. $\mathrm{H}_{2} \mathrm{O}$ & 3 eq. & 1 eq. & $26 \%$ \\
\hline Cyclohexane & 3 eq. & 1 eq. & Trace \\
\hline Ethanol & 3 eq. & 1 eq. & NR \\
\hline Tetrahydrofuran & 3 eq. & 1 eq. & NR \\
\hline
\end{tabular}

Table S2. Summary of results of the reaction conditions study.

\section{Preparation of amides:}

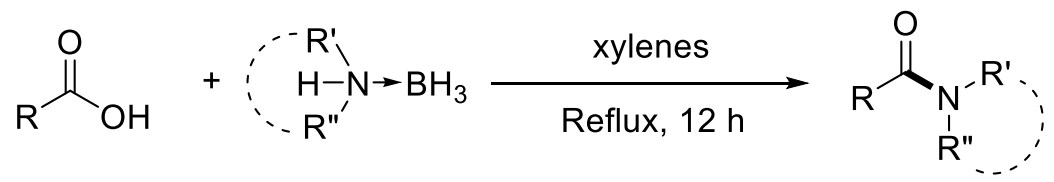

Scheme S7. Reaction equation for the preparation of amide products. 
In a $25 \mathrm{ml}$ round bottom flask containing a magnetic stir bar was weighed amine-borane $(2 \mathrm{mmol}$, 1 eq.) followed by carboxylic acid ( $6 \mathrm{mmol}, 3 \mathrm{eq})$. Xylenes was then added to the flask, $(6 \mathrm{~mL}, 1$ $\mathrm{M}$ with respect to carboxylic acid). A reflux condenser was attached to the flask and the reaction mixture was brought to reflux and monitored by ${ }^{11} \mathrm{~B}$ NMR. After completion ( 12 hours) the reaction mixture was diluted with methanol $(50 \mathrm{~mL})$ and condensed via rotary evaporation. The crude mixture was diluted with ethyl acetate $(15 \mathrm{~mL})$ and transferred to a separatory funnel. The mixture was washed with cold $3 \mathrm{M}$ sodium hydroxide solution $(3 \times 10 \mathrm{~mL})$, then washed with brine $(1 \times 15 \mathrm{~mL})$. The organic layer was dried with sodium sulfate, filtered through cotton, and condensed via rotary evaporation. Residual solvent was removed by placing under high-vacuum for $\sim 12 \mathrm{~h}$. Column chromatography was performed only if necessary to further purify the product. Modification for excess acid recovery: The combined aqueous $\mathrm{NaOH}$ extracts are acidified using $3 \mathrm{M} \mathrm{HCl}$ solution until acidic by $\mathrm{pH}$ paper. The aqueous mixture is extracted using ethyl acetate $(3 \times 20 \mathrm{~mL})$. The combined organic fractions are washed with brine $(1 \times 15 \mathrm{~mL})$, dried over sodium sulfate, filtered through cotton, and condensed via rotary evaporation. Residual solvent was removed by placing under high-vacuum for $\sim 12 \mathrm{~h}$.

\section{Procedures for mechanistic studies:}

Preparation of triacyloxyborane-amine complexes (4aa, $4 \mathrm{~cd})$ :

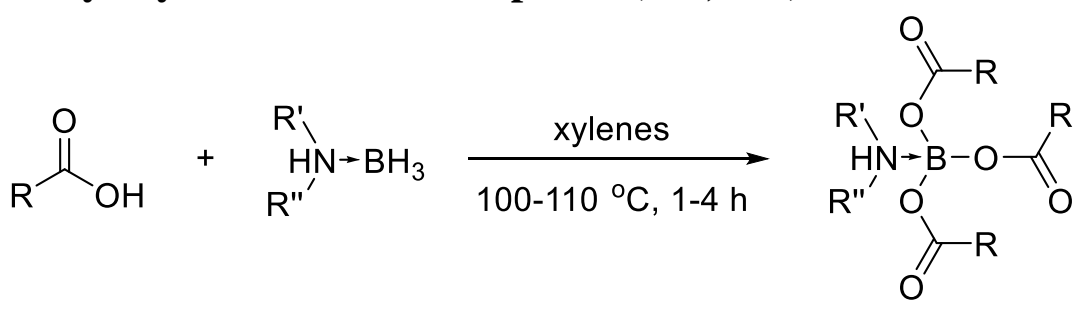

Scheme S8. Reaction equation for the preparation of amine-triacyloxyborane complexes.

In a $100 \mathrm{ml}$ round bottom flask containing a magnetic stir bar was weighed amine-borane (10 mmol, 1 eq.) followed by carboxylic acid $(30 \mathrm{mmol}, 3 \mathrm{eq})$. Xylenes was then added to the flask, (30 mL, $1 \mathrm{M}$ with respect to carboxylic acid). The reaction mixture was heated to $100-110{ }^{\circ} \mathrm{C}$ and stirred for 1-4 hours depending on substrate. After completion the reaction mixture was first allowed to cool to room temperature, then placed in a $0{ }^{\circ} \mathrm{C}$ freezer overnight. The precipitated solid was collected by filtration through a Büchner funnel and rinsed with cold hexanes. The solid material was transferred to a dry $100 \mathrm{~mL}$ round bottom flask, sealed, and residual solvent was removed by placing under high-vacuum for $\sim 12 \mathrm{~h}$. Both synthesized complexes were isolated in near quantitative yield. 


\section{Preparation of post-reaction complex :}

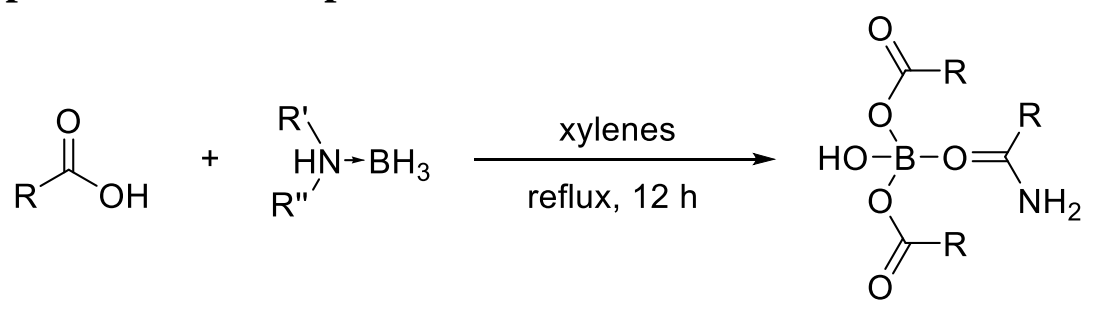

Scheme S9. Reaction equation for the preparation of post-reaction complex.

In a $100 \mathrm{ml}$ round bottom flask containing a magnetic stir bar was weighed amine-borane (10 mmol, 1 eq.) followed by carboxylic acid (30 mmol, $3 \mathrm{eq})$. Xylenes was then added to the flask, (30 $\mathrm{mL}, 1 \mathrm{M}$ with respect to carboxylic acid). A reflux condenser was attached to the flask and the reaction mixture was brought to reflux and monitored by TLC. After completion the reaction mixture was first allowed to cool to room temperature, then placed in a $0{ }^{\circ} \mathrm{C}$ freezer overnight. The precipitated solid was collected by filtration through a Büchner funnel and rinsed with cold hexanes. The solid material was transferred to a dry $100 \mathrm{~mL}$ round bottom flask, sealed, and residual solvent was removed by placing under high-vacuum for $\sim 12 \mathrm{~h}$.

\section{Amidation monitored by hydride analysis:}

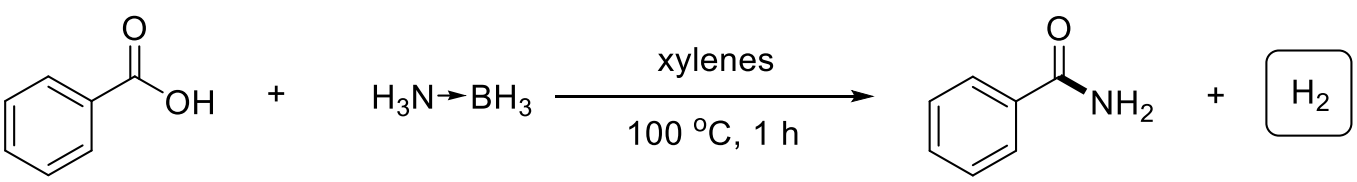

Scheme S10. Reaction equation for the preparation of post-reaction complex.

In a $25 \mathrm{ml}$ round bottom flask containing a magnetic stir bar was weighed ammonia-borane ( 2 mmol, 1 eq., $0.0617 \mathrm{~g}$ ) followed by benzoic acid ( $6 \mathrm{mmol}, 3 \mathrm{eq}, 0.7327 \mathrm{~g})$. Xylenes was then added to the flask, (6 mL, $1 \mathrm{M}$ with respect to carboxylic acid). A reflux condenser was attached to the flask, sealed with vacuum grease and Teflon tape. A barbed tygon hose adaptor was sttached to the top of the reflux condenser and sealed with vacuum grease and Teflon tape. An analytical gas buret was connect to the barbed adaptor of the reaction apparatus using tygon tubing. The reaction mixture was then brought to $100{ }^{\circ} \mathrm{C}$ and the amount of gas being evolved was monitored. After completion ( 1 hour) the reaction mixture was diluted with methanol $(50 \mathrm{~mL})$ and condensed via rotary evaporation. The crude mixture was diluted with ethyl acetate $(15 \mathrm{~mL})$ and transferred to a separatory funnel. The mixture was washed with cold $3 \mathrm{M}$ sodium hydroxide solution $(3 \mathrm{x} 10$ $\mathrm{mL})$, then washed with brine $(1 \times 15 \mathrm{~mL})$. The organic layer was dried with sodium sulfate, filtered through cotton, and condensed via rotary evaporation. Residual solvent was removed by placing under high-vacuum for $\sim 12 \mathrm{~h}$. 
Cross reaction of triacyloxyborane-amine complexes:

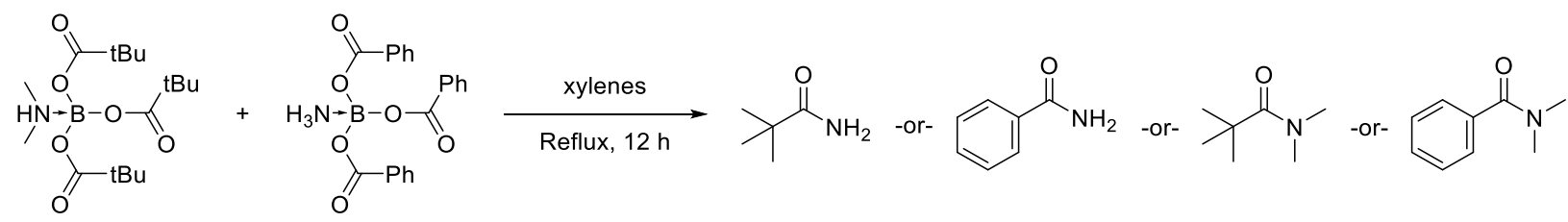

Scheme S11. Reaction equation for the cross reaction of amine-triacyloxyborane complexes.

In a $50 \mathrm{ml}$ round bottom flask containing a magnetic stir bar was weighed triacyloxyborane-amine 4aa ( 2 mmol, 1 eq., $0.7823 \mathrm{~g}$ ) followed by triacyloxyborane-amine 4cd ( 2 mmol, 1 eq, $0.7327 \mathrm{~g}$ ). Xylenes was then added to the flask, ( $4 \mathrm{~mL}, 0.5 \mathrm{M}$ w.r.t. either complex). A reflux condenser was attached to the flask and a reflux condenser were sealed with rubber septa. The reaction mixture was then brought to reflux and allowed to react for 12 hours. After completion the reaction mixture was diluted with methanol $(50 \mathrm{~mL})$ and condensed via rotary evaporation. Residual solvent was removed by placing under high-vacuum for $\sim 12 \mathrm{~h}$.
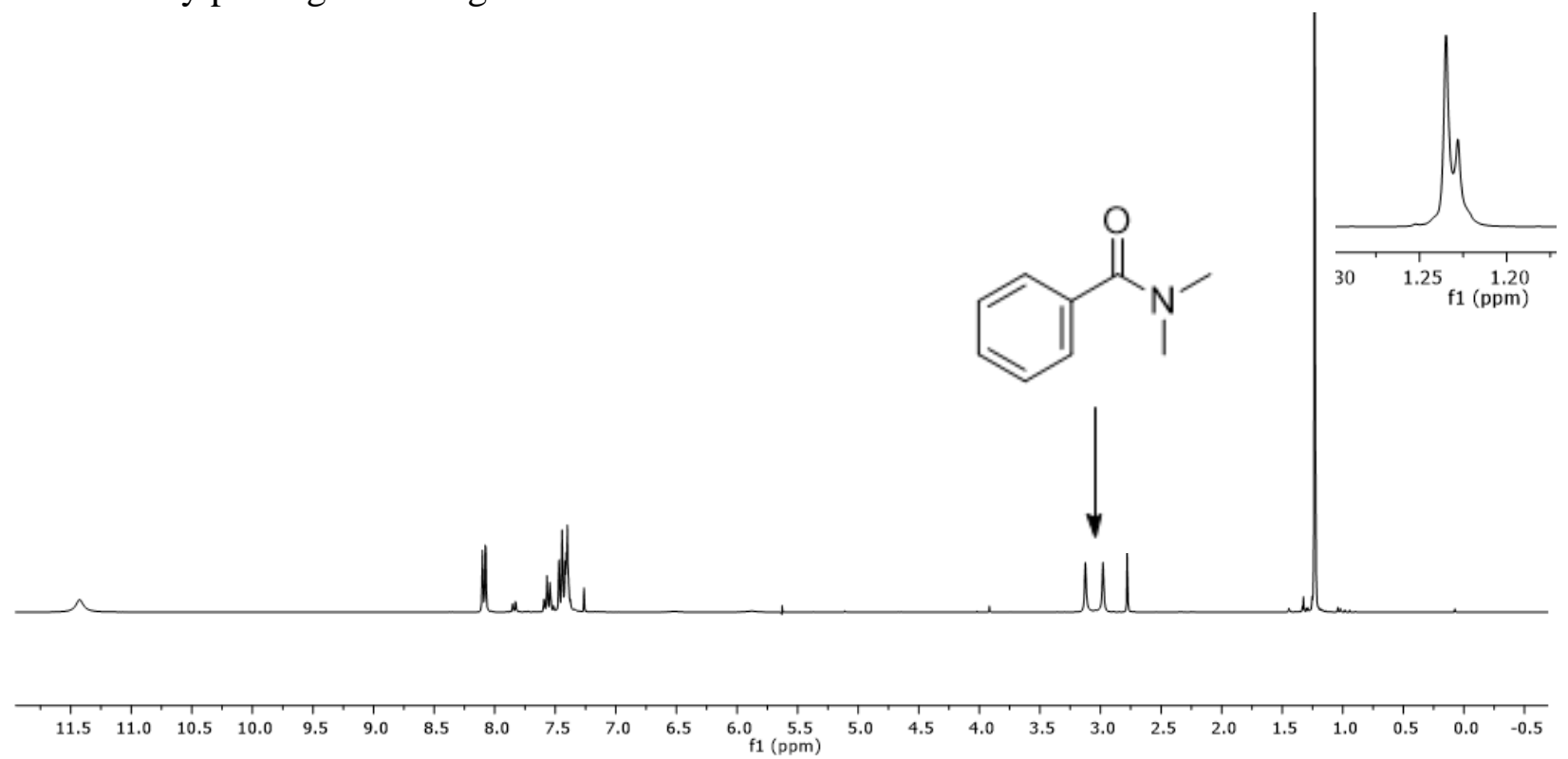

${ }^{1} \mathrm{H}$ NMR (300 MHz, Chloroform- $d$ ) cross reaction of amine-triacyloxyborane complexes. 


\section{Crystallographic information for 4aa}

Preparation of triacyloxyborane-amine complex 4aa sample for crystallographic analysis:

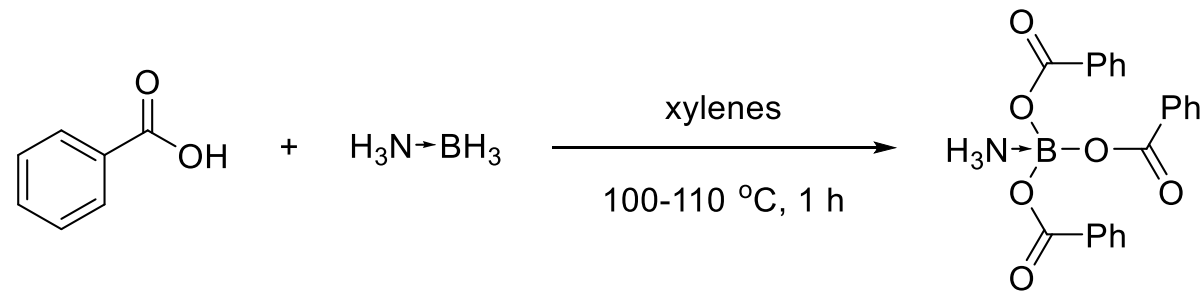

Scheme S12. Reaction equation for the preparation of the ammonia-tribenzyloxyborane complex.

In a $100 \mathrm{ml}$ round bottom flask containing a magnetic stir bar was weighed ammonia-borane (10 mmol, 1 eq., $0.3087 \mathrm{~g}$ ) followed by benzoic acid (30 mmol, $3 \mathrm{eq}, 3.6636 \mathrm{~g}$ ). Xylenes was then added to the flask, (30 mL, $1 \mathrm{M}$ w.r.t. carboxylic acid). The reaction mixture was heated to 100$110{ }^{\circ} \mathrm{C}$ and stirred for 1 hour. After completion the reaction mixture was first allowed to cool to room temperature, turning off the stir plate and oil bath and leaving the flask in the bath to slowly equilibrate to r.t. Once completely cooled to r.t. the flask was sealed and placed in a $0{ }^{\circ} \mathrm{C}$ freezer overnight. A sample of the precipitated solid was removed from the flask and placed into a $20 \mathrm{~mL}$ vial with a portion of the xylenes supernatant, this sample was subjected to x-ray crystallographic analysis. The crystallographic structure is shown below, see the attached CIF file for full structural details.
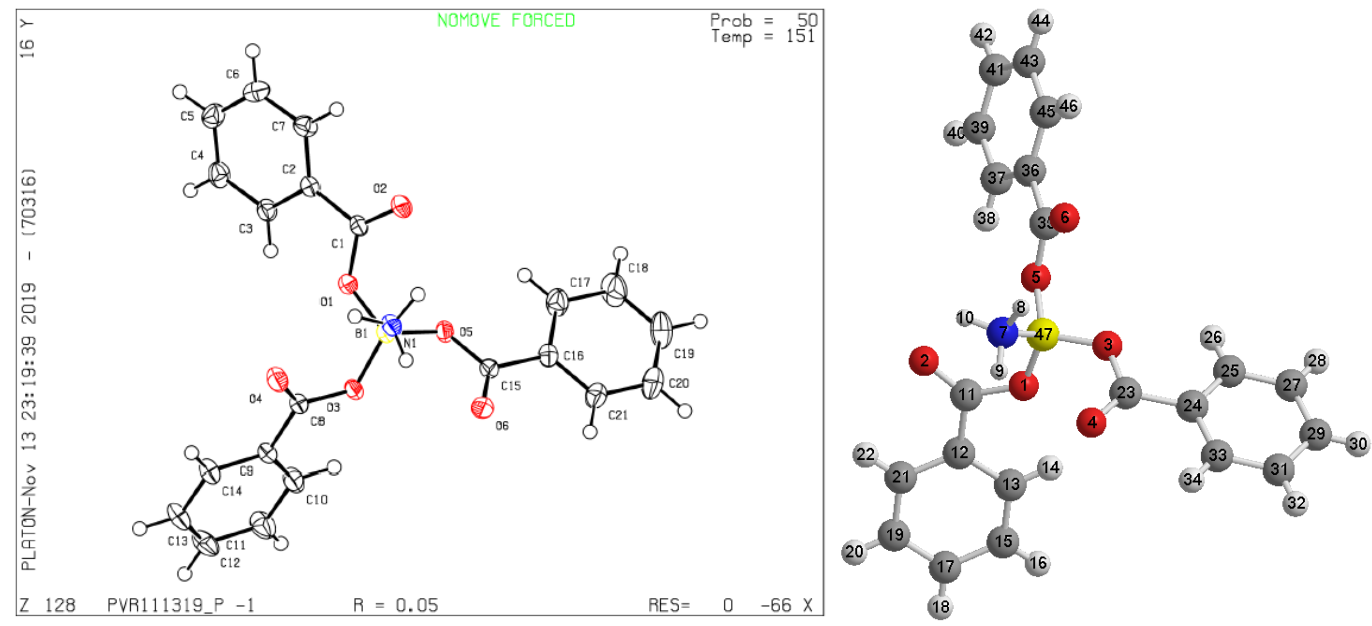

Figure S1. Crystal structure of the ammonia-tribenzyloxyborane complex. 


\section{Characterization of ammonia-borane and amine-boranes:}

\section{Ammonia-borane (1a):}

$4 \mathrm{~h}, \mathrm{THF}$,

$\mathrm{H}_{3} \mathrm{~N} \rightarrow \mathrm{BH}_{3} \quad$ procedure ia

$$
66 \%(10.185 \mathrm{~g})
$$

White solid.

${ }^{\mathbf{1}} \mathbf{H}$ NMR (300 MHz, Tetrahydrofuran- $\left.d 8\right) \delta 4.26-3.69(\mathrm{~m}, 3 \mathrm{H}), 2.08-0.80(\mathrm{~m}, 3 \mathrm{H}) .{ }^{11} \mathbf{B}$ NMR (96 MHz, Tetrahydrofuran- $d 8) \delta-22.04$ (q, $J=95.6 \mathrm{~Hz}$ ).

Characterization is in agreement with previous reports for this compound. ${ }^{2}$

\section{Methylamine-borane (1b):}

$$
\begin{array}{cc}
-\mathrm{N} \rightarrow \mathrm{BH}_{3} & 4 \mathrm{~h}, \mathrm{THF}, \\
\mathrm{H}_{2} & \text { procedure ib } \\
& 88 \%(0.790 \mathrm{~g})
\end{array}
$$

White solid.

${ }^{1}$ H NMR (300 MHz, Chloroform- $d$ ) $\delta 3.78(\mathrm{~s}, 2 \mathrm{H}), 2.54(\mathrm{t}, J=6.3 \mathrm{~Hz}, 3 \mathrm{H}), 1.49$ (q, $J=91.9 \mathrm{~Hz}$, $3 \mathrm{H}) .{ }^{13} \mathbf{C}$ NMR $\left(75 \mathrm{MHz}\right.$, Chloroform- $d$ ) $\delta 34.7 .{ }^{11} \mathbf{B}$ NMR (96 MHz, Chloroform- $d$ ) $\delta-18.78$ (q, $J=96.1 \mathrm{~Hz}$ ).

Characterization is in agreement with previous reports for this compound. ${ }^{6}$

\section{Dimethylamine-borane (1c):}

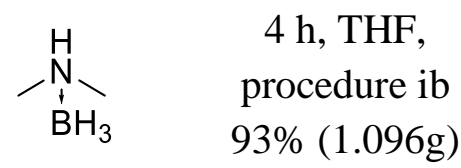

White solid.

${ }^{1}$ H NMR (300 MHz, Chloroform- $d$ ) $\delta 4.30(\mathrm{~s}, 1 \mathrm{H}), 2.46(\mathrm{~d}, J=5.8 \mathrm{~Hz}, 6 \mathrm{H}), 1.42$ (dd, $J=188.2$, $91.9 \mathrm{~Hz}, 3 \mathrm{H}) .{ }^{13} \mathrm{C}$ NMR $(75 \mathrm{MHz}$, Chloroform- $d) \delta$ 44.4. ${ }^{11} \mathbf{B}$ NMR $(96 \mathrm{MHz}$, Chloroform- $d$ ) $\delta$ $14.76(\mathrm{q}, J=95.5 \mathrm{~Hz})$.

Characterization is in agreement with previous reports for this compound. ${ }^{7}$

\section{Propylamine-borane (1d):}

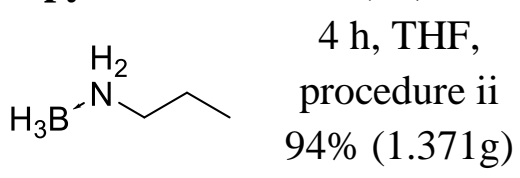

White solid.

${ }^{1}$ H NMR (300 MHz, Chloroform- $d$ ) $\delta 3.81$ (s, 2H), 2.74 (p, J= 7.2 Hz, 2H), $1.62(\mathrm{~h}, J=7.4 \mathrm{~Hz}$, 2H), 0.93 (t, $J=7.4 \mathrm{~Hz}, 3 \mathrm{H}) .{ }^{13} \mathbf{C}$ NMR (75 MHz, Chloroform- $d$ ) $\delta 50.5,22.4,11.1 .{ }^{11} \mathbf{B}$ NMR (96 $\mathrm{MHz}$, Chloroform- $d$ ) $\delta-19.84$ (q, $J=95.4 \mathrm{~Hz}$ ).

Characterization is in agreement with previous reports for this compound. ${ }^{4}$ 


\section{Tert-butylamine-borane (1e):}

\begin{tabular}{|c|c|}
\hline $\begin{array}{l}\mathrm{H}_{2} \mathrm{~N}^{-}-\mathrm{BH}_{3} \\
\mathrm{H}\end{array}$ & $\begin{array}{c}4 \mathrm{~h}, \mathrm{THF}, \\
\text { procedure ii }\end{array}$ \\
\hline & $94 \%(1.635 \mathrm{~g})$ \\
\hline
\end{tabular}

White solid.

${ }^{1}$ H NMR $\left(300 \mathrm{MHz}\right.$, Chloroform- $d$ ) $\delta 3.76$ (s, 2H), 1.27 (s, 9H). ${ }^{13} \mathbf{C}$ NMR $(75 \mathrm{MHz}$, Chloroformd) $\delta 53.2,28.0 .{ }^{11}$ B NMR $(96 \mathrm{MHz}$, Chloroform- $d) \delta-23.25$ (q, $\left.J=95.7 \mathrm{~Hz}\right)$.

Characterization is in agreement with previous reports for this compound. ${ }^{4}$

\section{Diethylamine-borane (1f):}

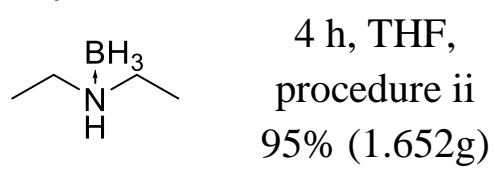

Colorless liquid.

${ }^{1}$ H NMR (300 MHz, Chloroform- $d$ ) $\delta 3.58(\mathrm{~s}, 1 \mathrm{H}), 2.72(\mathrm{qd}, J=7.3,5.6 \mathrm{~Hz}, 4 \mathrm{H}), 1.16(\mathrm{t}, J=7.3$ $\mathrm{Hz}, 6 \mathrm{H}) .{ }^{13} \mathbf{C}$ NMR $\left(75 \mathrm{MHz}\right.$, Chloroform- $d$ ) $\delta 48.8,11.6 .{ }^{11} \mathbf{B}$ NMR $(96 \mathrm{MHz}$, Chloroform- $d$ ) $\delta$ 17.44 (q, $J=95.9,95.4 \mathrm{~Hz})$.

Characterization is in agreement with previous reports for this compound. ${ }^{4}$

\section{Piperidine-borane (1g):}

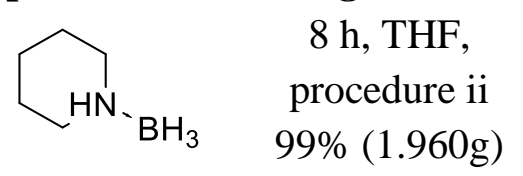

White solid.

${ }^{1}$ H NMR (300 MHz, Chloroform- $d$ ) $\delta 3.75(\mathrm{~s}, 1 \mathrm{H}), 3.32-3.13$ (m, 2H), 2.47 (tdd, $J=13.5,11.3$, $2.7 \mathrm{~Hz}, 2 \mathrm{H}), 1.79-1.73(\mathrm{~m}, 2 \mathrm{H}), 1.63-1.23(\mathrm{~m}, 4 \mathrm{H}) .{ }^{13} \mathbf{C}$ NMR $(75 \mathrm{MHz}$, Chloroform- $d$ ) $\delta 53.4$, 25.4, 22.6. ${ }^{11}$ B NMR $(96 \mathrm{MHz}$, Chloroform- $d$ ) $\delta-15.55$ (q, $J=95.4 \mathrm{~Hz})$.

Characterization is in agreement with previous reports for this compound. ${ }^{4}$

\section{Tert-octylamine-borane (1h):}

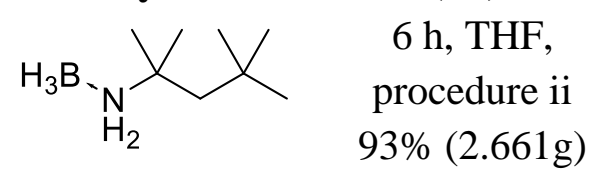

White solid.

${ }^{1} \mathbf{H}$ NMR (300 MHz, Chloroform- $d$ ) $\delta 3.70(\mathrm{~s}, 2 \mathrm{H}), 1.62(\mathrm{~s}, 2 \mathrm{H}), 1.33(\mathrm{~s}, 6 \mathrm{H}), 1.03(\mathrm{~s}, 9 \mathrm{H}) .{ }^{13} \mathbf{C}$ NMR $\left(75 \mathrm{MHz}\right.$, Chloroform- $d$ ) $\delta 57.0,52.4,31.5,27.5 .{ }^{11} \mathbf{B}$ NMR $(96 \mathrm{MHz}$, Chloroform- $d$ ) $\delta$ $23.46(\mathrm{q}, J=96.4 \mathrm{~Hz})$. 


\section{Cyclohexylamine-borane (1i):}

$$
ح^{\mathrm{N}^{\mathrm{H}_{2}}} \mathrm{BH}_{3} \quad \begin{gathered}
4 \mathrm{~h}, \mathrm{THF}, \\
\text { procedure ii } \\
98 \%(2.215 \mathrm{~g})
\end{gathered}
$$

White solid.

${ }^{1}$ H NMR (300 MHz, Chloroform- $d$ ) $\delta 4.03-3.25(\mathrm{~m}, 2 \mathrm{H}), 2.68(\mathrm{ttt}, J=10.2,6.4,3.8 \mathrm{~Hz}, 1 \mathrm{H})$, $2.12(\mathrm{dt}, J=12.4,3.7 \mathrm{~Hz}, 2 \mathrm{H}), 1.75(\mathrm{dq}, J=12.3,3.7,3.2 \mathrm{~Hz}, 2 \mathrm{H}), 1.62(\mathrm{dt}, J=12.3,3.7 \mathrm{~Hz}, 1 \mathrm{H})$, 1.37 - $1.10(\mathrm{~m}, 5 \mathrm{H}) .{ }^{13} \mathbf{C}$ NMR $(75 \mathrm{MHz}$, Chloroform- $d) \delta 57.0,32.4,25.4,24.6 .{ }^{11} \mathbf{B}$ NMR (96 $\mathrm{MHz}$, Chloroform- $d$ ) $\delta-20.90$ (q, $J=96.5,95.8 \mathrm{~Hz}$ ).

Characterization is in agreement with previous reports for this compound. ${ }^{4}$

\section{Benzylamine-borane (1j):

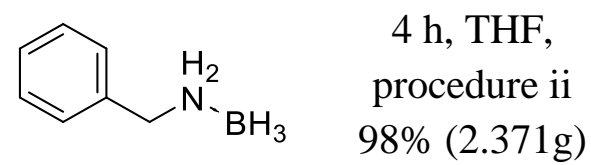

White solid.

${ }^{1}$ H NMR (300 MHz, Chloroform- $d$ ) $\delta 7.32$ (tdd, $\left.J=7.3,4.9,1.9 \mathrm{~Hz}, 5 \mathrm{H}\right), 4.22$ (s, 2H), $3.98-3.81$ $(\mathrm{m}, 2 \mathrm{H}), 2.45-0.93(\mathrm{~m}, 3 \mathrm{H}) .{ }^{13} \mathbf{C}$ NMR $(75 \mathrm{MHz}$, Chloroform- $d$ ) $\delta 135.9,129.1,128.6,128.4$, 53.0. ${ }^{11}$ B NMR (96 MHz, Chloroform- $d$ ) $\delta-19.76$ (q, $J=102.1 \mathrm{~Hz}$ ).

Characterization is in agreement with previous reports for this compound. ${ }^{4}$

\section{1-phenylethan-1-amine-borane (1k):}

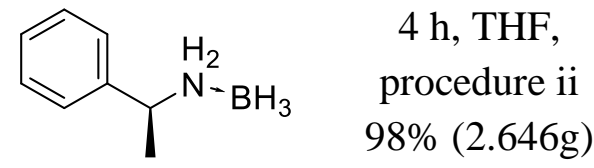

White solid.

${ }^{1}$ H NMR (300 MHz, Chloroform- $d$ ) $\delta 7.50-7.20(\mathrm{~m}, 5 \mathrm{H}), 3.96(\mathrm{dqd}, J=9.7,6.6,3.1 \mathrm{~Hz}, 3 \mathrm{H})$, $1.62(\mathrm{~d}, J=6.6 \mathrm{~Hz}, 3 \mathrm{H}) .{ }^{13} \mathrm{C}$ NMR $(75 \mathrm{MHz}$, Chloroform- $d) \delta 141.4,129.1,128.5,126.2,58.6$, 19.9. ${ }^{11}$ B NMR (96 MHz, Chloroform- $d$ ) $\delta-20.04(\mathrm{q}, J=100.1,98.4 \mathrm{~Hz})$.

\section{2,2,2-trifluoroethylamine-borane (11):}

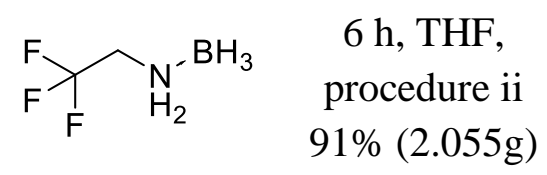

White solid.

${ }^{1}$ H NMR (300 MHz, Chloroform-d/ DMSO-d6) $\delta 6.02(\mathrm{~d}, J=11.3 \mathrm{~Hz}, 2 \mathrm{H}), 3.15$ (dq, $J=15.2$, $9.6 \mathrm{~Hz}, 2 \mathrm{H}), 1.32(\mathrm{~d}, J=70.1 \mathrm{~Hz}, 3 \mathrm{H}) .{ }^{13} \mathrm{C}$ NMR $(75 \mathrm{MHz}$, Chloroform- $d / \mathrm{DMSO}-d 6) \delta 129.0$ (q, $J=278.1 \mathrm{~Hz}), 53.4(\mathrm{q}, J=32.6 \mathrm{~Hz}) .{ }^{11} \mathbf{B}$ NMR $(96 \mathrm{MHz}$, Chloroform- $d /$ DMSO-d6) $\delta-17.76(\mathrm{q}$, $J=98.0 \mathrm{~Hz}) .{ }^{19} \mathbf{F}$ NMR $(282 \mathrm{MHz}$, Chloroform- $d /$ DMSO- $d \sigma) \delta-70.00$. 


\section{Morpholine-borane (1m):}

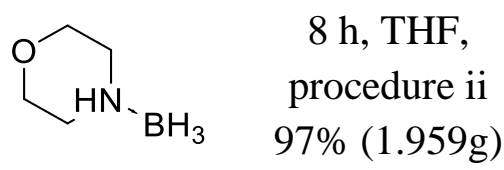

White solid.

${ }^{1}$ H NMR (300 MHz, Chloroform- $d$ ) $\delta 4.40(\mathrm{~s}, 1 \mathrm{H}), 3.91(\mathrm{dd}, J=12.7,3.6 \mathrm{~Hz}, 2 \mathrm{H}), 3.55(\mathrm{td}, J=$ $12.3,2.3 \mathrm{~Hz}, 2 \mathrm{H}), 3.12-2.96$ (m, 2H), 2.75 (dtd, $J=13.9,11.5,3.6 \mathrm{~Hz}, 2 \mathrm{H}), 1.44$ (d, $J=115.8$ $\mathrm{Hz}, 3 \mathrm{H}) .{ }^{13} \mathbf{C}$ NMR $\left(75 \mathrm{MHz}\right.$, Chloroform- $d$ ) $\delta 65.8,52.0 .{ }^{11} \mathbf{B}$ NMR $(96 \mathrm{MHz}$, Chloroform- $d$ ) $\delta$ -15.48 (q, $J=96.5 \mathrm{~Hz})$.

Characterization is in agreement with previous reports for this compound. ${ }^{4}$

\section{Allylamine-borane (1n):}

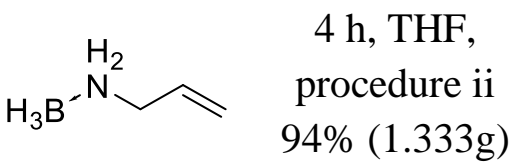

Colorless oil.

${ }^{1}$ H NMR (300 MHz, Chloroform- $d$ ) $\delta 5.88(\mathrm{ddt}, J=16.8,10.4,6.3 \mathrm{~Hz}, 1 \mathrm{H}), 5.34-5.13(\mathrm{~m}, 2 \mathrm{H})$, $4.08(\mathrm{~s}, 2 \mathrm{H}), 3.28$ (tdd, $J=7.8,5.8,1.3 \mathrm{~Hz}, 2 \mathrm{H}), 1.88-0.91(\mathrm{~m}, 3 \mathrm{H}) .{ }^{13} \mathrm{C}$ NMR $(75 \mathrm{MHz}$, Chloroform- $d$ ) $\delta 132.4,119.3,50.8 .{ }^{11} \mathbf{B}$ NMR $(96 \mathrm{MHz}$, Chloroform- $d$ ) $\delta$-19.90 (q, $J=95.2,94.0$ $\mathrm{Hz}$ ).Characterization is in agreement with previous reports for this compound. ${ }^{4}$

\section{Propargylamine-borane (10):

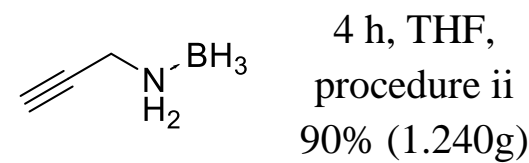

White solid.

${ }^{1} \mathbf{H}$ NMR $(300 \mathrm{MHz}, \mathrm{DMSO}-d 6) \delta 5.70(\mathrm{~s}, 2 \mathrm{H}), 3.30(\mathrm{~d}, J=29.1 \mathrm{~Hz}, 1 \mathrm{H}), 3.15(\mathrm{t}, J=6.9 \mathrm{~Hz}, 2 \mathrm{H})$, $1.24(\mathrm{~d}, J=103.9 \mathrm{~Hz}, 3 \mathrm{H}) .{ }^{13} \mathbf{C}$ NMR $(75 \mathrm{MHz}, \mathrm{DMSO}-d 6) \delta 80.1,76.0,36.7 .{ }^{11} \mathbf{B}$ NMR $(96 \mathrm{MHz}$, DMSO- $d 6$ ) $\delta-18.85$ (q, $J=119.7 \mathrm{~Hz})$.

Characterization is in agreement with previous reports for this compound. ${ }^{4}$

2-(4-nitrophenyl)ethan-1-amine-borane (1p):

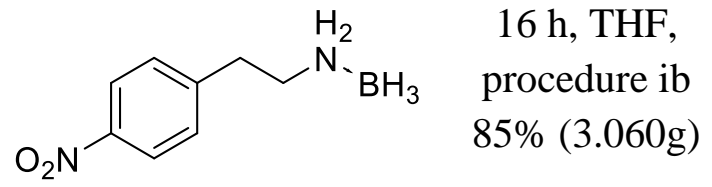

Orange solid.

${ }^{1}$ H NMR (300 MHz, DMSO- $d 6$ ) $\delta 8.24-7.94(\mathrm{~m}, 2 \mathrm{H}), 7.44(\mathrm{~d}, J=1.8 \mathrm{~Hz}, 2 \mathrm{H}), 5.33(\mathrm{~s}, 2 \mathrm{H}), 2.93$ $(\mathrm{dd}, J=9.5,6.4 \mathrm{~Hz}, 2 \mathrm{H}), 2.78-2.58(\mathrm{~m}, 2 \mathrm{H}), 1.32(\mathrm{~s}, 3 \mathrm{H}) .{ }^{13} \mathrm{C}$ NMR $(75 \mathrm{MHz}$, DMSO-d6) $\delta$ $152.1,151.2,134.9,128.6,53.5,39.2 .{ }^{11}$ B NMR (96 MHz, DMSO- $\left.d 6\right) \delta-19.80$ (q, $\left.J=112.2 \mathrm{~Hz}\right)$. Characterization is in agreement with previous reports for this compound. ${ }^{4}$ 


\section{2-aminoethane-1-thiol-borane (1q):}

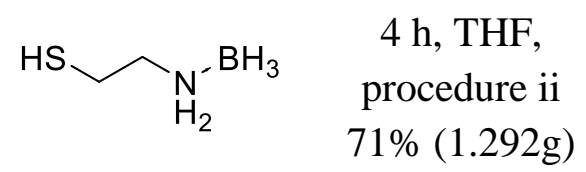

Pale yellow solid.

${ }^{1}$ H NMR (300 MHz, DMSO-d6) $\delta 4.52(\mathrm{~s}, 2 \mathrm{H}), 2.55-2.11(\mathrm{~m}, 5 \mathrm{H}), 0.79(\mathrm{~m}, 3 \mathrm{H}) .{ }^{13} \mathbf{C} \mathbf{N M R}(75$ MHz, DMSO-d6) $\delta 51.9,40.3 .{ }^{11} \mathbf{B}$ NMR (96 MHz, DMSO-d6) $\delta-19.92$ (q, $J=95.0,94.3 \mathrm{~Hz}$ ).

\section{Tyramine-borane (1r):}

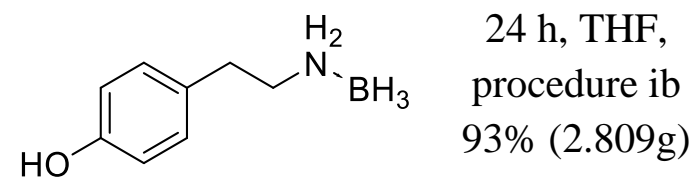

White solid.

${ }^{1}$ H NMR (300 MHz, DMSO-dO) $\delta 6.91(\mathrm{~d}, J=8.1 \mathrm{~Hz}, 2 \mathrm{H}), 6.64(\mathrm{~d}, J=7.9 \mathrm{~Hz}, 2 \mathrm{H}), 5.21(\mathrm{~s}, 2 \mathrm{H})$, $2.65(\mathrm{dd}, J=9.9,4.9 \mathrm{~Hz}, 2 \mathrm{H}), 2.60-2.51(\mathrm{~m}, 2 \mathrm{H}), 2.51-2.43(\mathrm{~m}, 1 \mathrm{H}), 1.25(\mathrm{~s}, 3 \mathrm{H}) .{ }^{13} \mathbf{C}$ NMR (75 MHz, DMSO-d6) $\delta$ 156.7, 129.1, 127.8, 115.5, 49.8, 33.5. ${ }^{11}$ B NMR (96 MHz, DMSO-d6) $\delta$ $-20.55(\mathrm{q}, J=95.3 \mathrm{~Hz})$.

\section{Valinol-borane (1s):

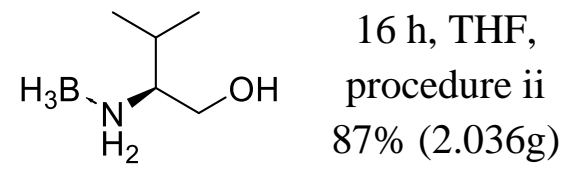

Colorless oil.

${ }^{1}$ H NMR (300 MHz, Chloroform- $d$ ) $\delta 4.17(\mathrm{~s}, 1 \mathrm{H}), 4.01(\mathrm{~d}, J=11.7 \mathrm{~Hz}, 1 \mathrm{H}), 3.73-3.66(\mathrm{~m}$, $1 \mathrm{H}), 2.65-2.55(\mathrm{~m}, 1 \mathrm{H}), 2.48(\mathrm{~s}, 2 \mathrm{H}), 2.10(\mathrm{dp}, J=13.9,6.9 \mathrm{~Hz}, 1 \mathrm{H}), 0.98(\mathrm{dd}, J=21.5,6.8$ $\mathrm{Hz}, 6 \mathrm{H}) .{ }^{13} \mathbf{C}$ NMR $\left(75 \mathrm{MHz}\right.$, Chloroform- $d$ ) $\delta$ 63.99, 58.75, 27.47, 19.53, 18.06. ${ }^{11} \mathbf{B}$ NMR (96 $\mathrm{MHz}$, Chloroform- $d$ ) $\delta-21.27$ (q, $J=98.1,96.2 \mathrm{~Hz}$ ).

\section{Aniline-borane (1t):}

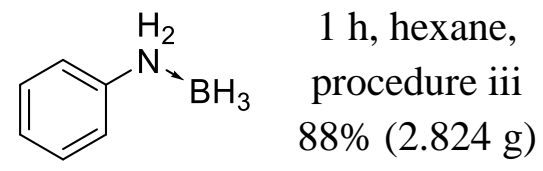

White solid.

${ }^{1}$ H NMR (300 MHz, Chloroform- $d$ ) $\delta 7.80-6.89$ (m, 5H), 5.44 (s, 2H), $2.55-1.27$ (m, 3H). ${ }^{13}$ C NMR (75 MHz, Chloroform- $d /$ DMSO- $\left.d 6\right) \delta 147.92,133.58,130.41,127.01 .{ }^{11}$ B NMR $(96$ $\mathrm{MHz}$, Chloroform- $d$ ) $\delta-16.17$ (q, $J=99.2,97.3 \mathrm{~Hz}$ ).

Characterization is in agreement with previous reports for this compound. ${ }^{5}$ 


\section{Characterization of amide products:}

\section{Benzamide (3aa):}

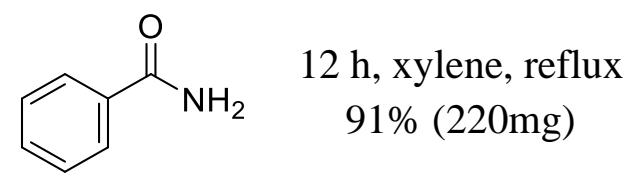

White solid, Melting point: $128-130^{\circ} \mathrm{C}$ (Meltemp),

$\mathrm{Rf}=0.31$ in hexanes:ethyl acetate 20:80 (UV, $\left.\mathrm{I}_{2}\right)$

${ }^{1}$ H NMR (300 MHz, DMSO-d6) $\delta 7.96(\mathrm{~s}, 1 \mathrm{H}), 7.90-7.81(\mathrm{~m}, 2 \mathrm{H}), 7.55-7.39(\mathrm{~m}, 3 \mathrm{H}), 7.36$ (s, 1H). ${ }^{13}$ C NMR (75 MHz, DMSO-d6) $\delta 172.9$, 139.3, 136.3, 133.3, 132.5.

HRMS (ESI) $m / z$ : $[\mathrm{M}+\mathrm{H}]$ calculated for $\mathrm{C}_{7} \mathrm{H}_{8} \mathrm{NO} 122.0606$, found 122.0609 .

Characterization is in agreement with previous reports for this compound. ${ }^{8,9,16}$

\section{Cyclohexanecarboxamide (3ab):}

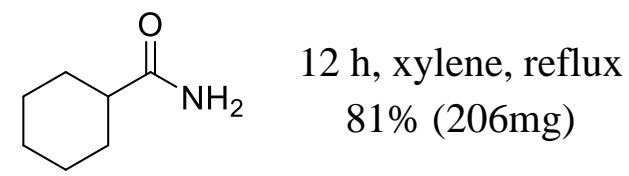

White solid, Melting point: $185-187^{\circ} \mathrm{C}$ (Meltemp),

$\mathrm{Rf}=0.32$ in hexanes:ethyl acetate $20: 80\left(\mathrm{UV}, \mathrm{I}_{2}\right)$

${ }^{1}$ H NMR (300 MHz, DMSO-d6) $\delta 5.54(\mathrm{~d}, J=37.5 \mathrm{~Hz}, 2 \mathrm{H}), 2.14(\mathrm{tt}, J=11.6,3.5 \mathrm{~Hz}, 1 \mathrm{H}), 1.90$ (dqd, $J=12.4,3.1,1.7 \mathrm{~Hz}, 2 \mathrm{H}$ ), 1.79 (ddt, $J=12.2,6.8,2.7 \mathrm{~Hz}, 2 \mathrm{H}$ ), 1.67 (dddd, $J=10.5,5.3$, $3.2,1.9 \mathrm{~Hz}, 1 \mathrm{H}), 1.42(\mathrm{qd}, J=12.0,2.5 \mathrm{~Hz}, 2 \mathrm{H}), 1.35-1.16(\mathrm{~m}, 3 \mathrm{H}) .{ }^{13} \mathbf{C}$ NMR $(75 \mathrm{MHz}, \mathrm{DMSO}-$ d6) $\delta 182.4,48.9,34.5,30.8,30.6$. HRMS (ESI) $m / z:[\mathrm{M}+\mathrm{H}]$ calculated for $\mathrm{C}_{7} \mathrm{H}_{14} \mathrm{NO} 128.1075$, found 128.1076 .

Characterization is in agreement with previous reports for this compound. ${ }^{9}$

\section{Hexanamide (3ac):}

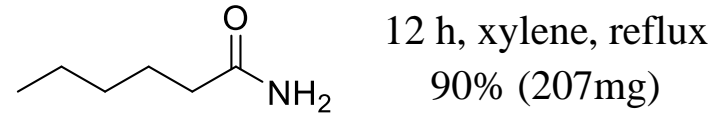

White solid, Melting point: $101-102^{\circ} \mathrm{C}$ (Meltemp),

$\mathrm{Rf}=0.17$ in hexanes:ethyl acetate 20:80 (UV, $\left.\mathrm{I}_{2}\right)$

${ }^{1}$ H NMR (300 MHz, Chloroform- $d$ ) $\delta 5.94(\mathrm{~d}, J=145.1 \mathrm{~Hz}, 2 \mathrm{H}), 2.22-2.13(\mathrm{~m}, 2 \mathrm{H}), 1.60$ (dq, $J=12.1,7.3 \mathrm{~Hz}, 2 \mathrm{H}), 1.28(\mathrm{pd}, J=7.2,4.5 \mathrm{~Hz}, 4 \mathrm{H}), 0.97-0.72(\mathrm{~m}, 3 \mathrm{H}) .{ }^{13} \mathbf{C}$ NMR $(75 \mathrm{MHz}$, Chloroform- $d$ ) $\delta 176.1,36.0,31.5,25.3,22.5,14.0$. HRMS (ESI) $m / z:[\mathrm{M}+\mathrm{H}]$ calculated for $\mathrm{C}_{6} \mathrm{H}_{14} \mathrm{NO}$ 116.1075, found 116.1083.

Characterization is in agreement with previous reports for this compound. ${ }^{10}$

\section{Pivalamide (3ad):}


$\overbrace{\mathrm{NH}_{2}} \begin{aligned} & 2 \mathrm{~h}, \text { xylene, reflux } \\ & 83 \%(168 \mathrm{mg})\end{aligned}$

White solid, Melting point: $154-156^{\circ} \mathrm{C}$ (Meltemp),

$\mathrm{Rf}=0.32$ in hexanes: ethyl acetate 20:80 (UV, $\left.\mathrm{I}_{2}\right)$

${ }^{1}$ H NMR (300 MHz, DMSO-d6) $\delta 6.85(\mathrm{~d}, J=90.1 \mathrm{~Hz}, 2 \mathrm{H}), 1.05(\mathrm{~s}, 9 \mathrm{H}) .{ }^{13} \mathrm{C}$ NMR $(75 \mathrm{MHz}$, DMSO-d6) $\delta 184.8,43.1,32.7$. HRMS (ESI) $m / z:[\mathrm{M}+\mathrm{H}]$ calculated for $\mathrm{C}_{5} \mathrm{H}_{12} \mathrm{NO} 102.0919$, found 102.0915.

Characterization is in agreement with previous reports for this compound. ${ }^{10}$

\section{2-phenylacetamide (3ae):}

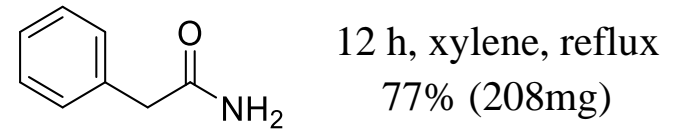

White solid, Melting point: $156-158^{\circ} \mathrm{C}$ (Meltemp),

$\mathrm{Rf}=0.26$ in hexanes:ethyl acetate 20:80 (UV, $\left.\mathrm{I}_{2}\right)$

${ }^{1}$ H NMR (300 MHz, DMSO-d6) $\delta 7.64-7.09(\mathrm{~m}, 5 \mathrm{H}), 5.66(\mathrm{~d}, J=137.8 \mathrm{~Hz}, 2 \mathrm{H}), 3.57(\mathrm{~s}, 2 \mathrm{H})$.

${ }^{13}$ C NMR (75 MHz, DMSO- $\left.d 6\right) \delta$ 177.5, 141.5, 134.2, 133.3, 131.4, 47.6. HRMS (ESI) $m / z$ : $[\mathrm{M}+\mathrm{H}]$ calculated for $\mathrm{C}_{8} \mathrm{H}_{10} \mathrm{NO} 136.0762$, found 136.0762 .

Characterization is in agreement with previous reports for this compound. ${ }^{9,10}$

\section{N-methylbenzamide (3ba):}

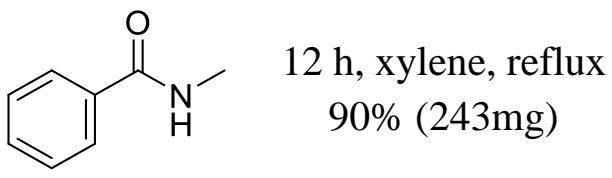

White solid, Melting point: $79-81^{\circ} \mathrm{C}$ (Meltemp), $\mathrm{Rf}=0.38$ in hexanes: ethyl acetate 20:80 (UV, $\left.\mathrm{I}_{2}\right)$

${ }^{1}$ H NMR (300 MHz, Chloroform-d) $\delta 7.90-7.68(\mathrm{~m}, 2 \mathrm{H}), 7.53-7.30$ (m, 3H), 6.73 (s, 1H), 2.95 $(\mathrm{d}, J=4.8 \mathrm{~Hz}, 3 \mathrm{H}) .{ }^{13} \mathbf{C}$ NMR $(75 \mathrm{MHz}$, Chloroform- $d$ ) $\delta$ 168.3, 134.5, 131.2, 128.4, 126.8, 26.9. HRMS (ESI) $m / z$ : $[\mathrm{M}+\mathrm{H}]$ calculated for $\mathrm{C}_{8} \mathrm{H}_{10} \mathrm{NO} 136.0762$, found 136.0770.

Characterization is in agreement with previous reports for this compound. ${ }^{10,16,17}$

\section{N-methylcyclohexanecarboxamide (3bb):}

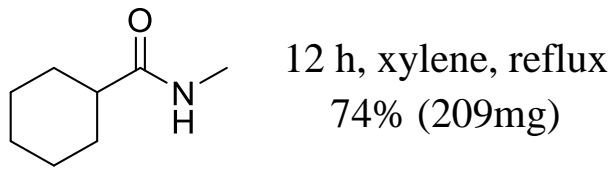

White solid, Melting point: $111-113^{\circ} \mathrm{C}$ (Meltemp),

Rf $=0.77$ in hexanes: ethyl acetate $30: 70\left(\mathrm{UV}, \mathrm{I}_{2}\right)$

${ }^{1}$ H NMR (300 MHz, Chloroform- $d$ ) $\delta 6.05(\mathrm{~s}, 1 \mathrm{H}), 2.73(\mathrm{~d}, J=4.8 \mathrm{~Hz}, 3 \mathrm{H}), 2.05(\mathrm{tt}, J=11.8,3.4$ $\mathrm{Hz}, 1 \mathrm{H}), 1.89-1.53(\mathrm{~m}, 5 \mathrm{H}), 1.38(\mathrm{tt}, J=12.1,6.0 \mathrm{~Hz}, 2 \mathrm{H}), 1.31-1.04(\mathrm{~m}, 3 \mathrm{H}) .{ }^{\mathbf{1 3}} \mathbf{C}$ NMR $(75$ MHz, Chloroform- $d$ ) $\delta 176.8,45.5,29.7,26.2$, 25.8. HRMS (ESI) $m / z:[\mathrm{M}+\mathrm{H}]$ calculated for $\mathrm{C}_{8} \mathrm{H}_{16} \mathrm{NO}$ 142.1232, found 142.1231.

Characterization is in agreement with previous reports for this compound. ${ }^{11}$ 


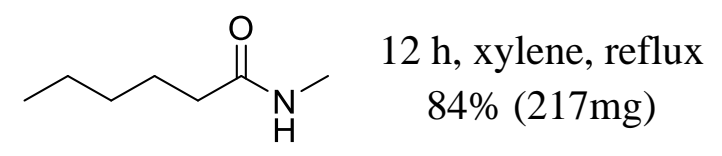

Slightly yellow liquid

$\mathrm{Rf}=0.78$ in hexanes:ethyl acetate $30: 70\left(\mathrm{UV}, \mathrm{I}_{2}\right)$

${ }^{1}$ H NMR (300 MHz, Chloroform- $d$ ) $\delta 6.00(\mathrm{~s}, 1 \mathrm{H}), 2.75(\mathrm{~d}, J=4.8 \mathrm{~Hz}, 3 \mathrm{H}), 2.21-2.06(\mathrm{~m}, 2 \mathrm{H})$, $1.67-1.52(\mathrm{~m}, 2 \mathrm{H}), 1.30-1.21(\mathrm{~m}, 4 \mathrm{H}), 0.93-0.79(\mathrm{~m}, 3 \mathrm{H}) .{ }^{13} \mathbf{C}$ NMR (75 MHz, Chloroformd) $\delta 173.9,36.7,31.6,26.3,25.6,22.5,14.0$. HRMS (ESI) $m / z$ : $[\mathrm{M}+\mathrm{H}]$ calculated for $\mathrm{C}_{7} \mathrm{H}_{16} \mathrm{NO}$ 130.1232, found 130.1229 .

Characterization is in agreement with previous reports for this compound. ${ }^{10}$

\section{4-chloro-2,5-difluoro-N-methylbenzamide (3bf):}

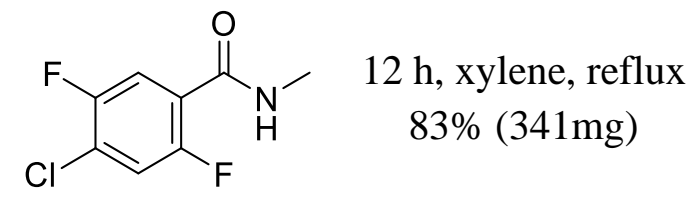

White solid, Melting point: $128-130^{\circ} \mathrm{C}$ (Meltemp),

$\mathrm{Rf}=0.50$ in hexanes:ethyl acetate $30: 70\left(\mathrm{UV}, \mathrm{I}_{2}\right)$

${ }^{1}$ H NMR (300 MHz, Chloroform- $d$ ) $\delta 7.79(\mathrm{dd}, J=9.3,6.6 \mathrm{~Hz}, 1 \mathrm{H}), 7.16(\mathrm{dd}, J=10.5,5.7 \mathrm{~Hz}$, $1 \mathrm{H}), 6.81(\mathrm{~s}, 1 \mathrm{H}), 2.97(\mathrm{dd}, J=4.8,1.1 \mathrm{~Hz}, 3 \mathrm{H}) .{ }^{13} \mathrm{C}$ NMR $(75 \mathrm{MHz}$, Chloroform- $d$ ) $\delta 161.7$, $156.6(\mathrm{~d}, J=61.3 \mathrm{~Hz}), 153.3(\mathrm{~d}, J=61.6 \mathrm{~Hz}), 125.0(\mathrm{dd}, J=20.5,11.9 \mathrm{~Hz}), 121.1(\mathrm{dd}, J=14.3$, $6.1 \mathrm{~Hz}), 118.6(\mathrm{dd}, J=25.7,3.6 \mathrm{~Hz}), 118.0,27.0 .{ }^{19}$ F NMR $(282 \mathrm{MHz}$, Chloroform- $d) \delta-119.00$, -120.91. HRMS (ESI) $m / z$ : $[\mathrm{M}+\mathrm{H}]$ calculated for $\mathrm{C}_{8} \mathrm{H}_{7} \mathrm{ClF}_{2} \mathrm{NO} 206.0184$, found 206.0189.

Characterization is in agreement with previous reports for this compound. ${ }^{12}$

\section{N,N-dimethylbenzamide (3ca):}

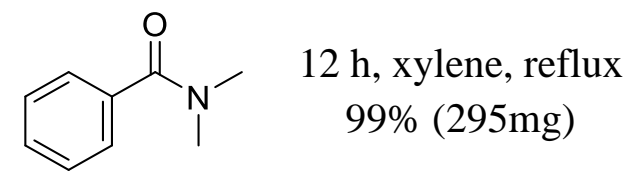

White solid, Melting point: $41-43^{\circ} \mathrm{C}$ (Meltemp),

Rf $=0.41$ in hexanes:ethyl acetate 20:80 (UV, $\left.\mathrm{I}_{2}\right)$

${ }^{1} \mathbf{H}$ NMR (300 MHz, Chloroform- $d$ ) $\delta 7.31$ (q, $\left.J=4.8,4.4 \mathrm{~Hz}, 5 \mathrm{H}\right), 2.94(\mathrm{~d}, J=42.2 \mathrm{~Hz}, 6 \mathrm{H}) .{ }^{13} \mathbf{C}$ NMR (75 MHz, Chloroform- $d) \delta 171.3,136.2,129.3,128.2,126.9,39.6,35.3$.

HRMS (ESI) $m / z$ : [M+H] calculated for $\mathrm{C}_{9} \mathrm{H}_{12} \mathrm{NO} 150.0919$, found 150.0914.

Characterization is in agreement with previous reports for this compound. ${ }^{13,14,19}$

\section{N,N-dimethylcyclohexanecarboxamide (3cb):}<smiles>CN(C)C(=O)C1CCCCC1</smiles>

$12 \mathrm{~h}$, xylene, reflux

$92 \%(286 \mathrm{mg})$

Clear, colorless liquid

$\mathrm{Rf}=0.30$ in hexanes:ethyl acetate $30: 70\left(\mathrm{UV}, \mathrm{I}_{2}\right)$

${ }^{1}$ H NMR (300 MHz, Chloroform- $d$ ) $\delta 3.05-2.89(\mathrm{~m}, 6 \mathrm{H}), 2.47(\mathrm{tt}, J=11.4,3.4 \mathrm{~Hz}, 1 \mathrm{H}), 1.82-$ $1.64(\mathrm{~m}, 5 \mathrm{H}), 1.48(\mathrm{qd}, J=11.7,3.1 \mathrm{~Hz}, 2 \mathrm{H}), 1.32-1.18(\mathrm{~m}, 3 \mathrm{H}) .{ }^{13} \mathrm{C}$ NMR $(75 \mathrm{MHz}$, 
Chloroform- $d$ ) $\delta 175.9,40.7,37.1,35.5,29.2,25.9$. HRMS (ESI) $m / z:[\mathrm{M}+\mathrm{H}]$ calculated for $\mathrm{C}_{9} \mathrm{H}_{18} \mathrm{NO}$ 156.1388, found 156.1388.

Characterization is in agreement with previous reports for this compound. ${ }^{14}$

\section{$\mathbf{N}, \mathbf{N}$-dimethylhexanamide (3cc):}

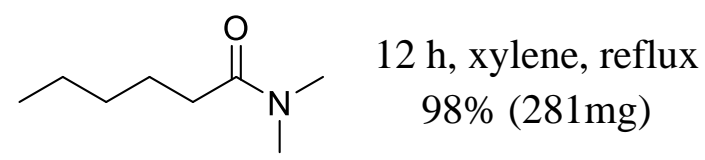

Slightly yellow liquid

$\mathrm{Rf}=0.33$ in hexanes:ethyl acetate $30: 70\left(\mathrm{UV}, \mathrm{I}_{2}\right)$

${ }^{1}$ H NMR (300 MHz, Chloroform- $d$ ) $\delta 2.98-2.83(\mathrm{~m}, 6 \mathrm{H}), 2.23(\mathrm{dd}, J=8.6,6.7 \mathrm{~Hz}, 2 \mathrm{H}), 1.64-$ $1.49(\mathrm{~m}, 2 \mathrm{H}), 1.25(\mathrm{tt}, J=10.2,4.4 \mathrm{~Hz}, 4 \mathrm{H}), 0.90-0.75(\mathrm{~m}, 3 \mathrm{H}) .{ }^{\mathbf{1 3}} \mathbf{C} \mathbf{N M R}(75 \mathrm{MHz}$, Chloroform-

d) $\delta 173.0,37.3,35.3,33.4,31.7,24.9,22.6,14.0$. HRMS (ESI) $m / z:[\mathrm{M}+\mathrm{H}]$ calculated for $\mathrm{C}_{8} \mathrm{H}_{18} \mathrm{NO}$ 144.1388, found 144.1396.

Characterization is in agreement with previous reports for this compound. ${ }^{15}$

\section{N-propylbenzamide (3da):}

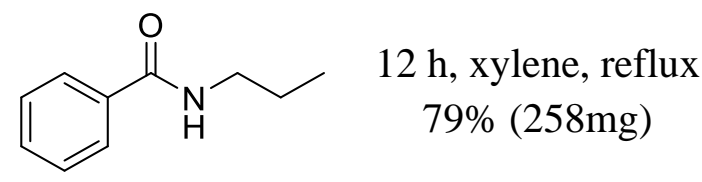

White solid, Melting point: $82-84^{\circ} \mathrm{C}$ (Meltemp), $\mathrm{Rf}=0.49$ in hexanes: ethyl acetate $30: 70\left(\mathrm{UV}, \mathrm{I}_{2}\right)$

${ }^{1}$ H NMR (300 MHz, Chloroform- $d$ ) $\delta 7.84-7.68$ (m, 2H), 7.47 - 7.38 (m, 1H), 7.38 - 7.29 (m, 2H), $6.84(\mathrm{~s}, 1 \mathrm{H}), 3.43-3.25(\mathrm{~m}, 2 \mathrm{H}), 1.58(\mathrm{~h}, J=7.4 \mathrm{~Hz}, 2 \mathrm{H}), 0.91(\mathrm{t}, J=7.4 \mathrm{~Hz}, 3 \mathrm{H}) .{ }^{13} \mathbf{C ~ N M R}$ (75 MHz, Chloroform- $d$ ) $\delta 167.6,134.7,131.1,128.3,126.9,41.8,23.0,11.6$.

HRMS (ESI) $m / z$ : $[\mathrm{M}+\mathrm{H}]$ calculated for $\mathrm{C}_{10} \mathrm{H}_{14} \mathrm{NO} 164.1075$, found 164.1076.

Characterization is in agreement with previous reports for this compound. ${ }^{16}$

\section{N-(tert-butyl)benzamide (3ea):}

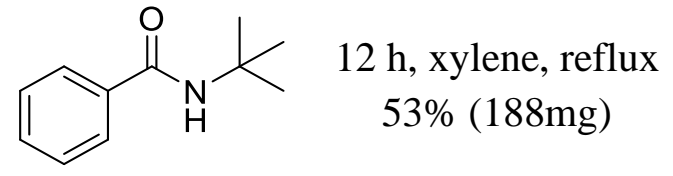

White solid, Melting point: $132-135^{\circ} \mathrm{C}$ (Meltemp),

$\mathrm{Rf}=0.80$ in hexanes:ethyl acetate $30: 70\left(\mathrm{UV}, \mathrm{I}_{2}\right)$

${ }^{1}$ H NMR (300 MHz, Chloroform- $d$ ) $\delta 7.76$ - 7.65 (m, 2H), 7.48 - 7.33 (m, 3H), 5.98 (s, 1H), 1.46 $(\mathrm{s}, 9 \mathrm{H}) .{ }^{13} \mathbf{C}$ NMR $(75 \mathrm{MHz}$, Chloroform- $d$ ) $\delta 166.8,135.8,131.0,128.4,126.6,51.7,29.0 . \mathbf{H R M S}$ (ESI) $m / z:[\mathrm{M}+\mathrm{Na}]$ calculated for $\mathrm{C}_{11} \mathrm{H}_{15} \mathrm{NONa} 200.1051$, found 200.1057 .

Characterization is in agreement with previous reports for this compound. ${ }^{17,} 19$

\section{N-(tert-butyl)cyclohexanecarboxamide (3eb):}

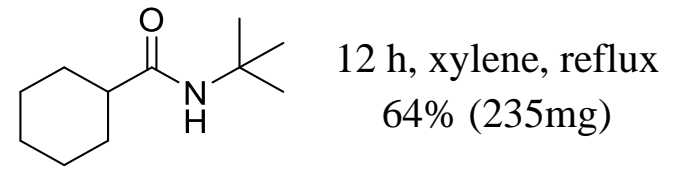

White solid, Melting point: $160-162^{\circ} \mathrm{C}$ (Meltemp), 
$\mathrm{Rf}=0.67$ in hexanes:ethyl acetate $30: 70\left(\mathrm{UV}, \mathrm{I}_{2}\right)$

${ }^{1}$ H NMR (300 MHz, Chloroform- $d$ ) $\delta 5.37(\mathrm{~s}, 1 \mathrm{H}), 1.89$ (tt, $\left.J=11.7,3.4 \mathrm{~Hz}, 1 \mathrm{H}\right), 1.71$ (tdd, $J=$ 11.5, 5.4, $2.7 \mathrm{~Hz}, 4 \mathrm{H}), 1.63-1.51(\mathrm{~m}, 1 \mathrm{H}), 1.44-1.29(\mathrm{~m}, 2 \mathrm{H}), 1.25(\mathrm{~s}, 9 \mathrm{H}), 1.22-1.08(\mathrm{~m}, 3 \mathrm{H})$. ${ }^{13}$ C NMR (75 MHz, Chloroform- $d$ ) $\delta 175.4,50.7,46.2,29.8,28.9,25.8$. HRMS (ESI) $m / z:[\mathrm{M}+\mathrm{H}]$ calculated for $\mathrm{C}_{11} \mathrm{H}_{22} \mathrm{NO}$ 184.1701, found 184.1696.

Characterization is in agreement with previous reports for this compound. ${ }^{18}$

\section{N,N-diethylbenzamide (3fa):}

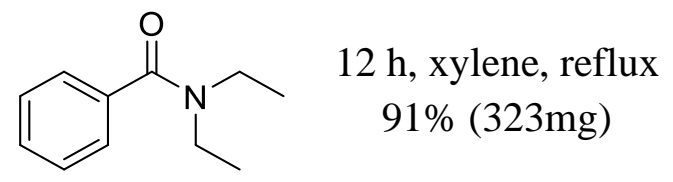

Slightly yellow liquid

Rf $=0.47$ in hexanes:ethyl acetate $30: 70\left(\mathrm{UV}, \mathrm{I}_{2}\right)$

${ }^{\mathbf{1}} \mathbf{H}$ NMR (300 MHz, Chloroform- $d$ ) $\delta 7.30(\mathrm{~s}, 5 \mathrm{H}), 3.53-3.08(\mathrm{~m}, 4 \mathrm{H}), 1.33-0.79(\mathrm{~m}, 6 \mathrm{H}) .{ }^{13} \mathbf{C}$ NMR (75 MHz, Chloroform- $d$ ) $\delta$ 171.1, 137.1, 129.0, 128.3, 126.1, 41.3 (d, $J=304.7 \mathrm{~Hz}), 13.7$ $\left(\mathrm{d}, J=97.2 \mathrm{~Hz}\right.$ ). HRMS (ESI) $m / z$ : $[\mathrm{M}+\mathrm{H}]$ calculated for $\mathrm{C}_{11} \mathrm{H}_{16} \mathrm{NO} 178.1232$, found 178.1230 .

Characterization is in agreement with previous reports for this compound. ${ }^{19}$

\section{Phenyl(piperidin-1-yl)methanone (3ga):}

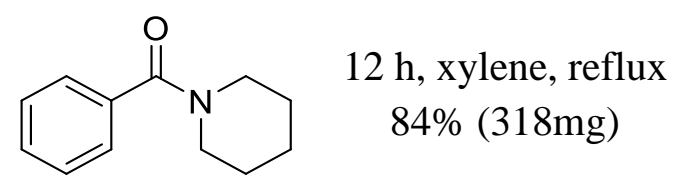

White solid, Melting point: $47-48^{\circ} \mathrm{C}$ (Meltemp)

$\mathrm{Rf}=0.46$ in hexanes:ethyl acetate 30:70 (UV, $\left.\mathrm{I}_{2}\right)$

${ }^{1}$ H NMR (300 MHz, Chloroform- $d$ ) $\delta 7.34$ (s, 5H), 3.48 (d, $\left.J=112.1 \mathrm{~Hz}, 4 \mathrm{H}\right), 1.63(\mathrm{~s}, 4 \mathrm{H}), 1.48$ (s, 2H). ${ }^{13}$ C NMR (75 MHz, Chloroform-d) $\delta 170.1,136.4,129.2,128.3,126.7,48.8,43.1,26.6$, 25.7, 24.7. HRMS (ESI) $m / z$ : [M+H] calculated for $\mathrm{C}_{12} \mathrm{H}_{16} \mathrm{NO} 190.1232$, found 190.1232 .

Characterization is in agreement with previous reports for this compound. ${ }^{19,21}$

\section{N-(2,4,4-trimethylpentan-2-yl)benzamide (3ha):}

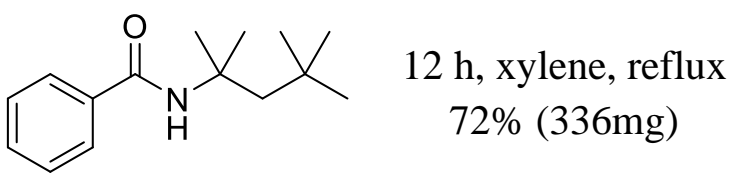

White solid, Melting point: $69-70^{\circ} \mathrm{C}$ (Meltemp)

$\mathrm{Rf}=0.70$ in hexanes:ethyl acetate $30: 70\left(\mathrm{UV}, \mathrm{I}_{2}\right)$

${ }^{1}$ H NMR (300 MHz, Chloroform- $d$ ) $\delta 7.74-7.65$ (m, 2H), 7.46 - 7.36 (m, 3H), $5.98(\mathrm{~s}, 1 \mathrm{H}), 1.86$ (s, 2H), 1.52 (s, 6H), 1.04 (s, 9H). ${ }^{13}$ C NMR (75 MHz, Chloroform- $d$ ) $\delta 166.5,136.1,130.9,128.4$, 126.5, 55.6, 51.7, 31.8, 31.7, 29.4. HRMS (ESI) $m / z:[\mathrm{M}+\mathrm{H}]$ calculated for $\mathrm{C}_{15} \mathrm{H}_{24} \mathrm{NO} 234.1858$, found 234.1855 .

Characterization is in agreement with previous reports for this compound. ${ }^{20}$ 
N-cyclohexylbenzamide (3ia):<smiles>O=C(NC1CCCCC1)c1ccccc1</smiles>

$12 \mathrm{~h}$, xylene, reflux

$89 \%(362 \mathrm{mg})$

White solid, Melting point: $148-150^{\circ} \mathrm{C}$ (Meltemp)

$\mathrm{Rf}=0.64$ in hexanes:ethyl acetate 20:80 $\left(\mathrm{UV}, \mathrm{I}_{2}\right)$

${ }^{1}$ H NMR (300 MHz, Chloroform- $d$ ) $\delta 7.74(\mathrm{dd}, J=7.2,1.7 \mathrm{~Hz}, 2 \mathrm{H}), 7.51-7.29(\mathrm{~m}, 3 \mathrm{H}), 6.35$ (d, $J=8.2 \mathrm{~Hz}, 1 \mathrm{H}), 4.04-3.79(\mathrm{~m}, 1 \mathrm{H}), 1.96(\mathrm{dt}, J=11.6,3.9 \mathrm{~Hz}, 2 \mathrm{H}), 1.71(\mathrm{dq}, J=12.7,3.7 \mathrm{~Hz}$, 2H), $1.60(\mathrm{dt}, J=12.5,3.7 \mathrm{~Hz}, 1 \mathrm{H}), 1.46-1.01(\mathrm{~m}, 5 \mathrm{H}) .{ }^{13} \mathrm{C}$ NMR $(75 \mathrm{MHz}$, Chloroform- $d) \delta$ 166.5, 135.0, 131.0, 128.3, 126.8, 48.8, 33.2, 25.6, 25.1. HRMS (ESI) $m / z:[\mathrm{M}+\mathrm{H}]$ calculated for $\mathrm{C}_{13} \mathrm{H}_{18} \mathrm{NO}^{+}$204.1388, found 204.1382

Characterization is in agreement with previous reports for this compound. ${ }^{21}$

\section{N-benzylbenzamide (3ja):}

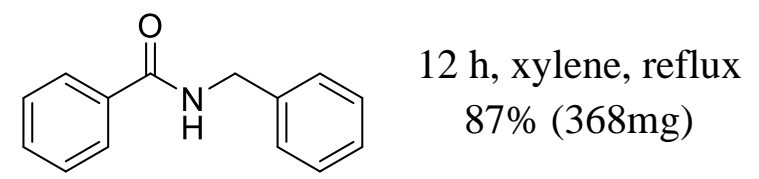

White solid, Melting point: $103-105^{\circ} \mathrm{C}$ (Meltemp)

$\mathrm{Rf}=0.55$ in hexanes:ethyl acetate $30: 70\left(\mathrm{UV}, \mathrm{I}_{2}\right)$

${ }^{1}$ H NMR (400 MHz, Chloroform- $d$ ) $\delta 7.86$ - 7.75 (m, 2H), 7.53 - 7.45 (m, 1H), 7.44 - 7.36 (m, 2H), $7.36-7.23(\mathrm{~m}, 5 \mathrm{H}), 6.73(\mathrm{~s}, 1 \mathrm{H}), 4.61(\mathrm{~d}, J=5.7 \mathrm{~Hz}, 2 \mathrm{H}) .{ }^{13} \mathrm{C}$ NMR (101 MHz, Chloroformd) $\delta 167.5,138.3,134.3,131.4,128.6,128.4,127.7,127.3,127.0,43.9$.

HRMS (ESI) $m / z$ : [M+H] calculated for $\mathrm{C}_{14} \mathrm{H}_{14} \mathrm{NO} 212.1075$, found 212.1071.

Characterization is in agreement with previous reports for this compound. ${ }^{16,19,21,23}$

\section{N-benzylcyclohexanecarboxamide (3jb):}

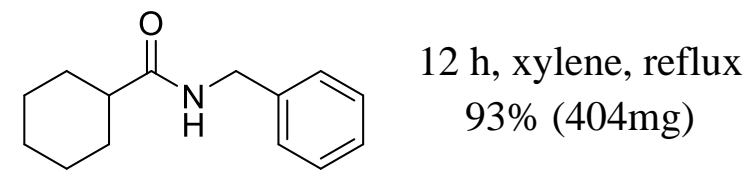

White solid, Melting point: $113-114^{\circ} \mathrm{C}$ (Meltemp)

$\mathrm{Rf}=0.52$ in hexanes:ethyl acetate $30: 70\left(\mathrm{UV}, \mathrm{I}_{2}\right)$

${ }^{1}$ H NMR (300 MHz, Chloroform- $d$ ) $\delta 7.39-7.01(\mathrm{~m}, 5 \mathrm{H}), 6.55(\mathrm{t}, J=5.9 \mathrm{~Hz}, 1 \mathrm{H}), 4.33(\mathrm{~d}, J=$ $5.8 \mathrm{~Hz}, 2 \mathrm{H}), 2.11(\mathrm{tt}, J=11.8,3.5 \mathrm{~Hz}, 1 \mathrm{H}), 1.97-1.56(\mathrm{~m}, 5 \mathrm{H}), 1.56-1.33(\mathrm{~m}, 2 \mathrm{H}), 1.21$ (tdd, $J$ $=18.1,12.4,6.1 \mathrm{~Hz}, 3 \mathrm{H}) .{ }^{13} \mathrm{C}$ NMR $(75 \mathrm{MHz}$, Chloroform- $d) \delta 176.2,138.7,128.4,127.4,127.1$, 45.4, 43.2, 29.8, 25.8. HRMS (ESI) $m / z$ : [M+H] calculated for $\mathrm{C}_{14} \mathrm{H}_{20} \mathrm{NO} 218.1545$, found 218.1544.

Characterization is in agreement with previous reports for this compound. ${ }^{22,23}$

\section{N-benzylhexanamide (3jc):}

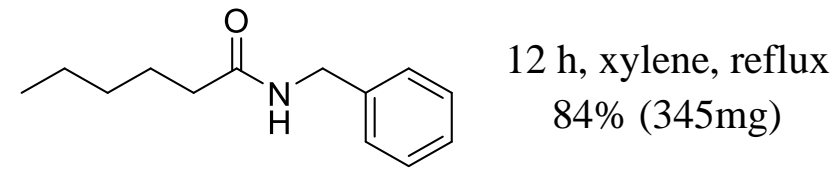

White solid, Melting point: $51-53^{\circ} \mathrm{C}$ (Meltemp) 
$\mathrm{Rf}=0.63$ in hexanes:ethyl acetate $30: 70\left(\mathrm{UV}, \mathrm{I}_{2}\right)$

${ }^{1}$ H NMR (300 MHz, Chloroform- $d$ ) $\delta 7.44-7.15(\mathrm{~m}, 5 \mathrm{H}), 6.29(\mathrm{~s}, 1 \mathrm{H}), 4.37$ (d, J = 5.7 Hz, 2H), $2.27-2.09(\mathrm{~m}, 2 \mathrm{H}), 1.73-1.51(\mathrm{~m}, 2 \mathrm{H}), 1.44-1.15(\mathrm{~m}, 4 \mathrm{H}), 1.02-0.79(\mathrm{~m}, 3 \mathrm{H}) .{ }^{13} \mathbf{C}$ NMR $(75$ MHz, Chloroform- $d$ ) $\delta 173.1,138.4,128.5,127.6,127.3,43.5,36.7,31.6,25.6,22.2,14.1$. HRMS (ESI) $m / z:[\mathrm{M}+\mathrm{H}]$ calculated for $\mathrm{C}_{13} \mathrm{H}_{20} \mathrm{NO} 206.1545$, found 206.1545 .

Characterization is in agreement with previous reports for this compound. ${ }^{23}$

\section{N-benzylpivalamide (3jd):}

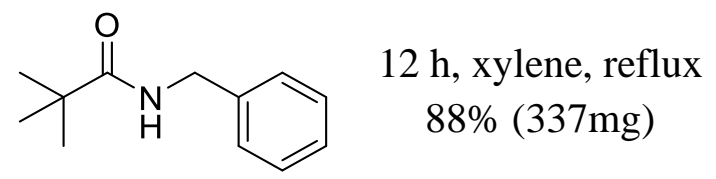

White solid, Melting point: $81-82^{\circ} \mathrm{C}$ (Meltemp)

$\mathrm{Rf}=0.69$ in hexanes:ethyl acetate $30: 70\left(\mathrm{UV}, \mathrm{I}_{2}\right)$

${ }^{1}$ H NMR (300 MHz, Chloroform- $d$ ) $\delta 7.35-7.15(\mathrm{~m}, 5 \mathrm{H}), 6.35(\mathrm{~s}, 1 \mathrm{H}), 4.36(\mathrm{~d}, J=5.8 \mathrm{~Hz}, 2 \mathrm{H})$, 1.19 (s, 9H). ${ }^{13} \mathrm{C}$ NMR (75 MHz, Chloroform- $d$ ) $\delta$ 178.2, 138.8, 128.5, 127.4, 127.1, 43.5, 38.7, 27.7. HRMS (ESI) $m / z:[\mathrm{M}+\mathrm{H}]$ calculated for $\mathrm{C}_{12} \mathrm{H}_{18} \mathrm{NO} 192.1388$, found 192.1383.

Characterization is in agreement with previous reports for this compound. ${ }^{23}$

\section{N-benzylacetamide (3jg):}

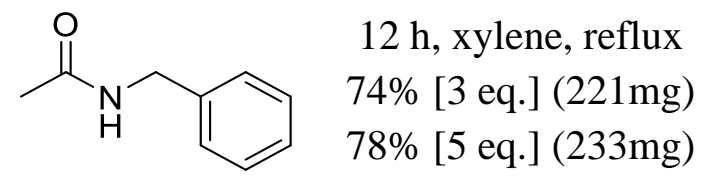

White solid, Melting point: $62-64^{\circ} \mathrm{C}$ (Meltemp)

$\mathrm{Rf}=0.30$ in hexanes:ethyl acetate $20: 80\left(\mathrm{UV}, \mathrm{I}_{2}\right)$

${ }^{1}$ H NMR (300 MHz, Chloroform-d) $\delta 7.45$ - 7.15 (m, 5H), 6.29 (s, 1H), 4.36 (d, J = 5.7 Hz, 2H), 1.96 (s, 3H). ${ }^{13} \mathrm{C}$ NMR (75 MHz, Chloroform- $d$ ) $\delta 170.0,138.2,128.6,127.7,127.4,43.7$, 23.3. HRMS (ESI) $m / z:[\mathrm{M}+\mathrm{H}]$ calculated for $\mathrm{C}_{9} \mathrm{H}_{12} \mathrm{NO} 150.0919$, found 150.0918 .

Characterization is in agreement with previous reports for this compound. ${ }^{24}$

\section{N-benzyl-2,2,2-trifluoroacetamide (3jh):}

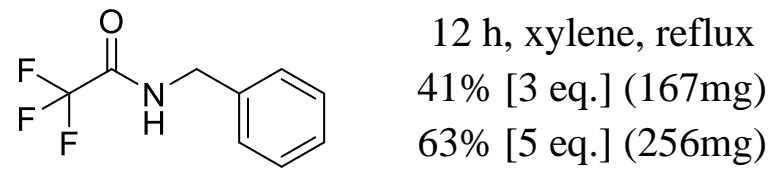

White solid, Melting point: $71-73^{\circ} \mathrm{C}$ (Meltemp) $\mathrm{Rf}=0.55$ in hexanes:ethyl acetate $30: 70\left(\mathrm{UV}, \mathrm{I}_{2}\right)$

${ }^{1}$ H NMR (300 MHz, Chloroform- $d$ ) $\delta 7.40-7.31(\mathrm{~m}, 3 \mathrm{H}), 7.31-7.15(\mathrm{~m}, 3 \mathrm{H}), 4.47$ (d, J = 5.9 $\mathrm{Hz}, 2 \mathrm{H}) .{ }^{13} \mathbf{C}$ NMR (75 MHz, Chloroform- $\left.d\right) \delta 157.3$ (q, $\left.J=36.9 \mathrm{~Hz}\right), 135.9,128.8,128.1,127.8$, 115.9 (q, $J=286.9 \mathrm{~Hz}), 43.9 .{ }^{19}$ F NMR $(282 \mathrm{MHz}$, Chloroform- $d$ ) $\delta-77.28$. HRMS (ESI) $m / z$ : $[\mathrm{M}+\mathrm{H}]$ calculated for $\mathrm{C}_{9} \mathrm{H}_{9} \mathrm{~F}_{3} \mathrm{NO} 204.0636$, found 204.0635.

Characterization is in agreement with previous reports for this compound. ${ }^{25}$ 


\section{N-benzylformamide (3ji):}

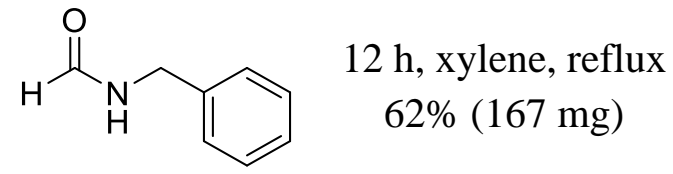

White solid, Melting point: $59-61^{\circ} \mathrm{C}$ (Meltemp),

$\mathrm{Rf}=0.16$ in hexanes:ethyl acetate 50:50 (UV, $\left.\mathrm{I}_{2}\right)$

${ }^{1}$ H NMR $(300 \mathrm{MHz}$, Chloroform- $d) \delta 8.09$ (s, $\left.0.87 \mathrm{H}\right), 7.99(\mathrm{~d}, J=11.9 \mathrm{~Hz}, 0.13 \mathrm{H}), 7.40-7.12$ $(\mathrm{m}, 5 \mathrm{H}), 6.88(\mathrm{~s}, 0.85 \mathrm{H}), 6.46(\mathrm{~s}, 0.15 \mathrm{H}), 4.35(\mathrm{~d}, J=6.0 \mathrm{~Hz}, 1.75 \mathrm{H}), 4.28$ (d, $J=6.5 \mathrm{~Hz}, 0.33$ H). ${ }^{13} \mathrm{C}$ NMR (75 MHz, Chloroform- $d$ ) $\delta 164.8$ (minor), 161.3 (major), 137.6 (major), 137.5 (minor), 128.8 (minor), 128.6 (major), 127.8 (minor), 127.6 (major), 127.4 (major), 126.9 (minor), 45.7 (minor), 42.1 (major). HRMS (ESI) $m / z$ : $[\mathrm{M}+\mathrm{H}]$ calculated for $\mathrm{C}_{8} \mathrm{H}_{10} \mathrm{NO} 136.0762$, found 136.0761 .

Characterization is in agreement with previous reports for this compound. ${ }^{26}$

(R)-N-benzyl-2-phenylbutanamide (3jj):

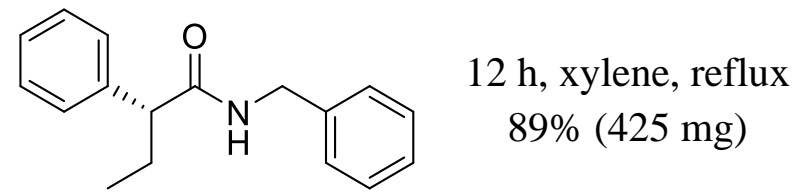

White solid, Melting point: $73-76^{\circ} \mathrm{C}$ (Meltemp), $\mathrm{Rf}=0.23$ in hexanes:ethyl acetate 20:80 (UV, $\left.\mathrm{I}_{2}\right)$

${ }^{1}$ H NMR (300 MHz, Chloroform- $d$ ) $\delta 7.35-7.22$ (m, 8H), $7.14(\mathrm{dd}, J=7.4,1.9 \mathrm{~Hz}, 2 \mathrm{H}), 5.97(\mathrm{~d}$, $J=5.9 \mathrm{~Hz}, 1 \mathrm{H}), 4.52-4.24(\mathrm{~m}, 2 \mathrm{H}), 3.29(\mathrm{t}, J=7.6 \mathrm{~Hz}, 1 \mathrm{H}), 2.36-2.12(\mathrm{~m}, 1 \mathrm{H}), 1.92-1.73(\mathrm{~m}$, 1H), $1.02-0.83(\mathrm{~m}, 3 \mathrm{H}) .{ }^{13} \mathbf{C}$ NMR (75 MHz, Chloroform-d) $\delta 139.9,138.3,128.7,128.5,127.9$, 127.4, 127.2, 127.1, 55.3, 43.6, 26.5, 12.6. HRMS (ESI) $m / z:[\mathrm{M}+\mathrm{H}]$ calculated for $\mathrm{C}_{17} \mathrm{H}_{20} \mathrm{NO}$ 254.1545, found 254.1548.

Characterization is in agreement with previous reports for this compound. ${ }^{27}$

\section{(S)-N-(1-phenylethyl)benzamide (3ka):}

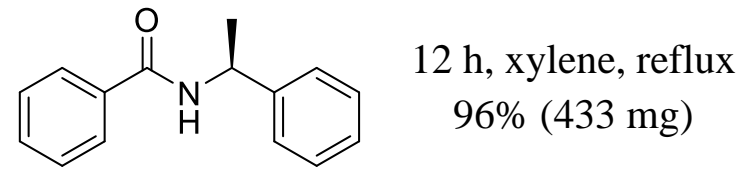

White solid, Melting point: $121-124^{\circ} \mathrm{C}$ (Meltemp), $\mathrm{Rf}=0.66$ in hexanes: ethyl acetate 20:80 (UV, $\left.\mathrm{I}_{2}\right)$

${ }^{1}$ H NMR (300 MHz, Chloroform- $d$ ) $\delta 7.90-7.74(\mathrm{~m}, 2 \mathrm{H}), 7.48-7.17$ (m, 9H), 5.31 (p, $J=7.0$ $\mathrm{Hz}, 1 \mathrm{H}), 1.64-1.47$ (m, 3H). ${ }^{13} \mathrm{C}$ NMR (75 MHz, Chloroform- $d$ ) $\delta 166.7,143.4,134.5,131.3$, 128.5, 128.4, 127.2, 126.2, 49.3, 21.9. HRMS (ESI) $m / z:[\mathrm{M}+\mathrm{H}]$ calculated for $\mathrm{C}_{15} \mathrm{H}_{16} \mathrm{NO}$ 226.1232 , found 226.1227 .

Characterization is in agreement with previous reports for this compound. ${ }^{21}$

\section{N-(2,2,2-trifluoroethyl)benzamide (3la):}

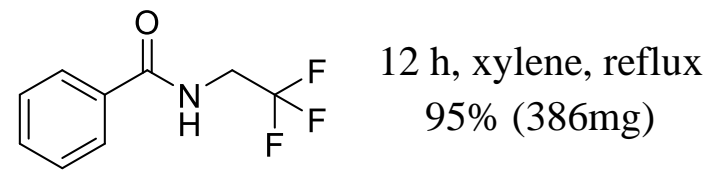


White solid, Melting point: $114-116^{\circ} \mathrm{C}$ (Meltemp),

$\mathrm{Rf}=0.62$ in hexanes:ethyl acetate $30: 70\left(\mathrm{UV}, \mathrm{I}_{2}\right)$

${ }^{1}$ H NMR (300 MHz, Chloroform- $d$ ) $\delta 7.90-7.72(\mathrm{~m}, 2 \mathrm{H}), 7.60-7.52(\mathrm{~m}, 1 \mathrm{H}), 7.47$ (tq, $J=6.6$, $1.2 \mathrm{~Hz}, 2 \mathrm{H}), 6.39$ (s, 1H), 4.14 (qdd, $J=9.1,6.5,0.8 \mathrm{~Hz}, 2 \mathrm{H}) .{ }^{13} \mathrm{C}$ NMR (75 MHz, Chloroformd) $\delta 167.8,133.1,132.1,128.6,127.1,41.1$ (q, $J=34.6,34.0 \mathrm{~Hz}) .{ }^{19} \mathrm{~F} \mathrm{NMR}(282 \mathrm{MHz}$, Chloroform- $d$ ) $\delta$-73.82. HRMS (ESI) $m / z$ : $[\mathrm{M}+\mathrm{H}]$ calculated for $\mathrm{C}_{9} \mathrm{H}_{9} \mathrm{~F}_{3} \mathrm{NO} 204.0636$, found 204.0644.

Characterization is in agreement with previous reports for this compound. ${ }^{28}$

\section{Morpholino(phenyl)methanone (3ma):}

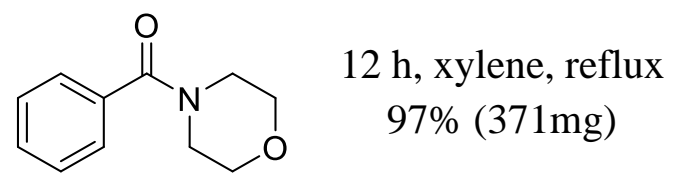

White solid, Melting point: $70-73^{\circ} \mathrm{C}$ (Meltemp),

$\mathrm{Rf}=0.41$ in hexanes:ethyl acetate $20: 80\left(\mathrm{UV}, \mathrm{I}_{2}\right)$

${ }^{1} \mathbf{H}$ NMR (300 MHz, Chloroform- $d$ ) $\delta 7.36(\mathrm{~s}, 5 \mathrm{H}), 3.55(\mathrm{~d}, J=79.9 \mathrm{~Hz}, 8 \mathrm{H}) .{ }^{13} \mathbf{C}$ NMR $(75 \mathrm{MHz}$, Chloroform- $d$ ) $\delta 170.2,135.2,129.8,128.5,127.0,66.9,48.4,42.5$.

HRMS (ESI) $m / z$ : $[\mathrm{M}+\mathrm{H}]$ calculated for $\mathrm{C}_{11} \mathrm{H}_{14} \mathrm{NO}_{2}$ 192.1025, found 192.1032.

Characterization is in agreement with previous reports for this compound. ${ }^{19,21}$

\section{N-allylcinnamamide (3nk):}

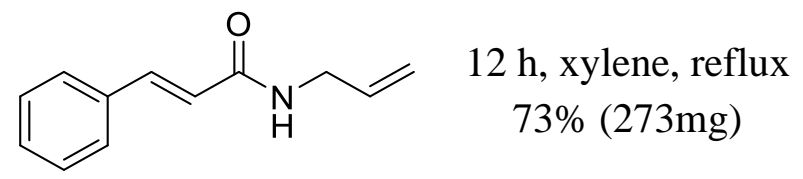

White solid, Melting point: $87-89^{\circ} \mathrm{C}$ (Meltemp),

$\mathrm{Rf}=0.44$ in hexanes:ethyl acetate $30: 70\left(\mathrm{UV}, \mathrm{I}_{2}\right)$

${ }^{1}$ H NMR (300 MHz, Chloroform- $d$ ) $\delta 7.64(\mathrm{~d}, J=15.7 \mathrm{~Hz}, 1 \mathrm{H}), 7.50-7.41(\mathrm{~m}, 2 \mathrm{H}), 7.33-7.24$ $(\mathrm{m}, 3 \mathrm{H}), 7.10(\mathrm{t}, J=5.8 \mathrm{~Hz}, 1 \mathrm{H}), 6.63(\mathrm{~d}, J=15.6 \mathrm{~Hz}, 1 \mathrm{H}), 5.98-5.80(\mathrm{~m}, 1 \mathrm{H}), 5.30-5.17(\mathrm{~m}$, 1H), $5.11(\mathrm{dd}, J=10.2,1.7 \mathrm{~Hz}, 1 \mathrm{H}), 4.06-3.93(\mathrm{~m}, 2 \mathrm{H}) .{ }^{13} \mathrm{C}$ NMR $(75 \mathrm{MHz}$, Chloroform- $d) \delta$ 166.2 140.7, 134.8, 134.0, 129.5, 128.7, 127.7, 120.9, 116.3, 42.3. HRMS (ESI) m/z: [M+H] calculated for $\mathrm{C}_{12} \mathrm{H}_{14} \mathrm{NO} 188.1075$, found 188.1074 .

Characterization is in agreement with previous reports for this compound. ${ }^{29}$

\section{N-(prop-2-yn-1-yl)cinnamamide (3ok):}

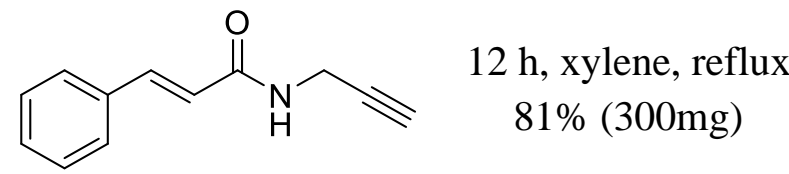

White solid, Melting point: $103-106^{\circ} \mathrm{C}$ (Meltemp),

$\mathrm{Rf}=0.48$ in hexanes:ethyl acetate $30: 70\left(\mathrm{UV}, \mathrm{I}_{2}\right)$

${ }^{1}$ H NMR (300 MHz, Chloroform- $\left.d\right) \delta 7.66(\mathrm{~d}, J=15.6 \mathrm{~Hz}, 1 \mathrm{H}), 7.55-7.47$ (m, 2H), 7.38 (dt, $J$ $=4.8,2.9 \mathrm{~Hz}, 3 \mathrm{H}), 6.39(\mathrm{~d}, J=15.6 \mathrm{~Hz}, 1 \mathrm{H}), 5.78(\mathrm{~s}, 1 \mathrm{H}), 4.20(\mathrm{dd}, J=5.3,2.6 \mathrm{~Hz}, 2 \mathrm{H}), 2.27(\mathrm{t}$, $J=2.5 \mathrm{~Hz}, 1 \mathrm{H}) .{ }^{13} \mathrm{C}$ NMR $(75 \mathrm{MHz}$, Chloroform- $d) \delta 165.6,141.7,134.5,129.8,128.7,127.8$, 119.9, 71.7, 29.6. HRMS (ESI) $m / z$ : [M+H] calculated for $\mathrm{C}_{12} \mathrm{H}_{12} \mathrm{NO} 186.0919$, found 186.0905. Characterization is in agreement with previous reports for this compound. ${ }^{30}$ 


\section{N-(4-nitrophenethyl)benzamide (3pa):}

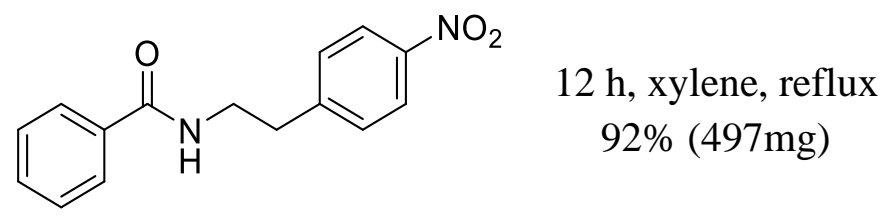

White solid, Melting point: $162-164^{\circ} \mathrm{C}$ (Meltemp), $\mathrm{Rf}=0.43$ in hexanes:ethyl acetate $20: 80\left(\mathrm{UV}, \mathrm{I}_{2}\right)$

${ }^{1}$ H NMR (300 MHz, Chloroform- $\left.d\right) \delta 8.13(\mathrm{~d}, J=8.4 \mathrm{~Hz}, 2 \mathrm{H}), 7.79-7.65(\mathrm{~m}, 2 \mathrm{H}), 7.53-7.46$ $(\mathrm{m}, 1 \mathrm{H}), 7.44-7.34(\mathrm{~m}, 4 \mathrm{H}), 6.44(\mathrm{~s}, 1 \mathrm{H}), 3.73(\mathrm{q}, J=6.7 \mathrm{~Hz}, 2 \mathrm{H}), 3.05(\mathrm{t}, J=7.0 \mathrm{~Hz}, 2 \mathrm{H}) .{ }^{13} \mathbf{C}$ NMR (75 MHz, Chloroform-d) $\delta$ 167.5, 146.8, 146.6, 134.1, 131.6, 129.6, 128.6, 126.7, 123.8, 40.9, 35.8. HRMS (ESI) $m / z:[\mathrm{M}+\mathrm{H}]$ calculated for $\mathrm{C}_{15} \mathrm{H}_{15} \mathrm{~N}_{2} \mathrm{O}_{3} 271.1083$, found 271.1079.

\section{N-(2-mercaptoethyl)-4-methoxybenzamide (3qi):}

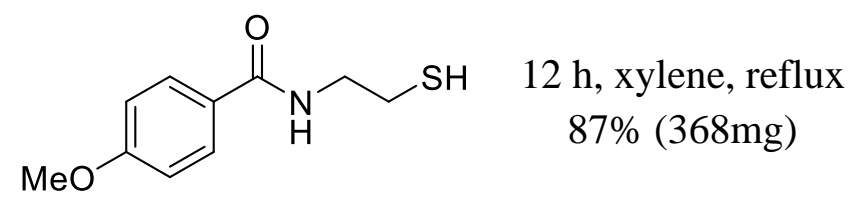

Pale yellow solid, Melting point: $87-90^{\circ} \mathrm{C}$ (Meltemp),

$\mathrm{Rf}=0.28$ in hexanes:ethyl acetate $20: 80\left(\mathrm{UV}, \mathrm{I}_{2}\right)$

${ }^{1}$ H NMR (300 MHz, Chloroform- $d$ ) $\delta 7.87-7.73(\mathrm{~m}, 2 \mathrm{H}), 7.01(\mathrm{~d}, J=6.3 \mathrm{~Hz}, 1 \mathrm{H}), 6.95-6.82$ $(\mathrm{m}, 2 \mathrm{H}), 3.83(\mathrm{~s}, 3 \mathrm{H}), 3.77(\mathrm{q}, J=6.2 \mathrm{~Hz}, 2 \mathrm{H}), 2.96(\mathrm{t}, J=6.4 \mathrm{~Hz}, 2 \mathrm{H}) .{ }^{13} \mathbf{C} \mathbf{N M R}(75 \mathrm{MHz}$, Chloroform- $d$ ) $\delta 167.2,162.1,128.8,126.3,113.7,55.5,39.3,38.2$.

HRMS (ESI) $m / z$ : [M+H] calculated for $\mathrm{C}_{10} \mathrm{H}_{14} \mathrm{NO}_{2} \mathrm{~S} 212.0745$, found 212.0753.

\section{N-(4-hydroxyphenethyl)benzamide (3ra):}

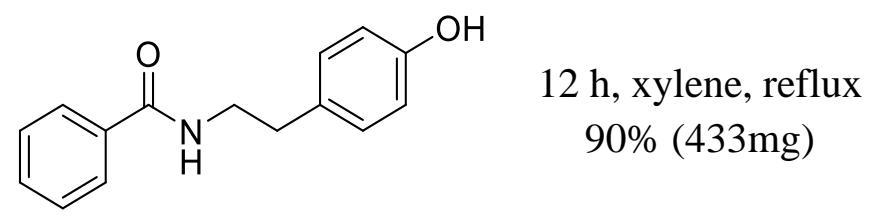

White solid, Melting point: $162-164^{\circ} \mathrm{C}$ (Meltemp), $\mathrm{Rf}=0.39$ in hexanes:ethyl acetate 20:80 (UV, $\left.\mathrm{I}_{2}\right)$

${ }^{1}$ H NMR (300 MHz, DMSO-d6) $\delta 9.17(\mathrm{~s}, 1 \mathrm{H}), 8.50(\mathrm{~d}, J=5.7 \mathrm{~Hz}, 1 \mathrm{H}), 7.80(\mathrm{~d}, J=7.2 \mathrm{~Hz}, 2 \mathrm{H})$, $7.45(\mathrm{dt}, J=13.9,6.7 \mathrm{~Hz}, 3 \mathrm{H}), 7.01(\mathrm{~d}, J=8.4 \mathrm{~Hz}, 2 \mathrm{H}), 6.66(\mathrm{~d}, J=8.4 \mathrm{~Hz}, 2 \mathrm{H}), 3.39(\mathrm{q}, J=6.7$ $\mathrm{Hz}, 2 \mathrm{H}), 2.70(\mathrm{t}, J=7.3 \mathrm{~Hz}, 2 \mathrm{H}) .{ }^{13} \mathrm{C}$ NMR (75 MHz, DMSO-d6) $\delta 171.2,160.7,139.8,136.1$, 134.6, 133.3, 132.2, 120.3, 46.6, 39.6. HRMS (ESI) $m / z$ : $[\mathrm{M}+\mathrm{H}]$ calculated for $\mathrm{C}_{15} \mathrm{H}_{16} \mathrm{NO}_{2}$ 242.1181 , found 242.1181 .

(S)-2-benzamido-3-methylbutyl benzoate (3sa):<smiles>CC(C)[C@H](COC(=O)c1ccccc1)NC(=O)c1ccccc1</smiles>

$12 \mathrm{~h}$, xylene, reflux $50 \%(311 \mathrm{mg})$

White solid, Melting point: $76-81^{\circ} \mathrm{C}$ (Meltemp), $\mathrm{Rf}=0.37$ in hexanes: ethyl acetate 50:50 (UV, $\left.\mathrm{I}_{2}\right)$ 
${ }^{1}$ H NMR (300 MHz, Chloroform- $d$ ) $\delta 8.15-7.92(\mathrm{~m}, 2 \mathrm{H}), 7.88-7.69(\mathrm{~m}, 2 \mathrm{H}), 7.52(\mathrm{t}, J=7.3$ $\mathrm{Hz}, 1 \mathrm{H}), 7.48-7.28(\mathrm{~m}, 5 \mathrm{H}), 6.56(\mathrm{~d}, J=8.8 \mathrm{~Hz}, 1 \mathrm{H}), 4.54(\mathrm{dd}, J=11.3,6.8 \mathrm{~Hz}, 1 \mathrm{H}), 4.48-$ $4.26(\mathrm{~m}, 2 \mathrm{H}), 2.21-1.91(\mathrm{~m}, J=6.8 \mathrm{~Hz}, 1 \mathrm{H}), 1.05(\mathrm{dd}, J=6.8,3.6 \mathrm{~Hz}, 6 \mathrm{H}) .{ }^{13} \mathrm{C} \mathrm{NMR}(75$ MHz, Chloroform- $d$ ) $\delta 167.42,166.68,134.58,133.07,131.31,129.66,129.53,128.45,128.34$, $126.83,65.18,54.14,30.04,19.53,18.97$. HRMS (ESI) $m / z:[\mathrm{M}+\mathrm{H}]$ calculated for $\mathrm{C}_{19} \mathrm{H}_{22} \mathrm{NO}_{3}$ 312.3890, found 312.3879.

\section{N-benzylisonicotinamide (3jm):}

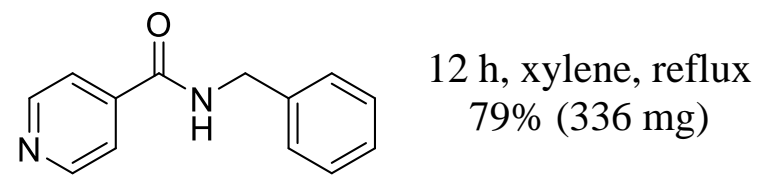

White solid, Melting point: $85-87^{\circ} \mathrm{C}$ (Meltemp), $\mathrm{Rf}=0.10$ in hexanes:ethyl acetate 50:50 (UV, $\left.\mathrm{I}_{2}\right)$

${ }^{1} \mathbf{H}$ NMR (300 MHz, Chloroform- $d$ ) $\delta 8.61(\mathrm{~d}, J=5.1 \mathrm{~Hz}, 2 \mathrm{H}), 7.65-7.55(\mathrm{~m}, 2 \mathrm{H}), 7.38-7.25$ $(\mathrm{m}, 5 \mathrm{H}), 7.25-7.13(\mathrm{~m}, 1 \mathrm{H}), 4.58(\mathrm{~d}, J=5.7 \mathrm{~Hz}, 2 \mathrm{H}) .{ }^{13} \mathbf{C}$ NMR $(75 \mathrm{MHz}$, Chloroform- $d) \delta$ 165.34, 150.17, 141.45, 137.44, 128.74, 127.79, 127.71, 121.01, 44.26. HRMS (ESI) $m / z$ : $[\mathrm{M}+\mathrm{H}]$ calculated for $\mathrm{C}_{13} \mathrm{H}_{13} \mathrm{~N}_{2} \mathrm{O} 213.1028$, found 213.1024 .

Characterization is in agreement with previous reports for this compound. ${ }^{31}$

\section{1-mercapto-1-morpholinoundecan-1-one (3mn):}

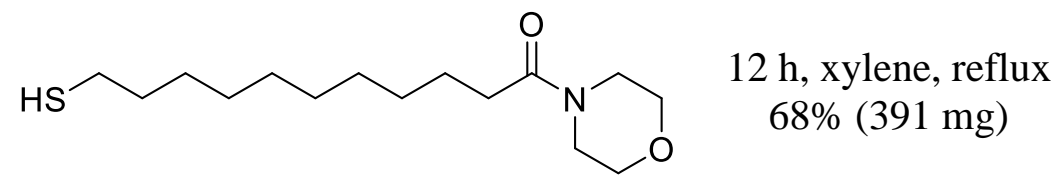

Clear, colorless oil

$\mathrm{Rf}=0.17$ in hexanes: ethyl acetate 50:50 $\left(\mathrm{I}_{2}\right)$

${ }^{1}$ H NMR (300 MHz, Chloroform- $d$ ) $\delta 3.70-3.60(\mathrm{~m}, 4 \mathrm{H}), 3.58(\mathrm{~d}, J=4.7 \mathrm{~Hz}, 2 \mathrm{H}), 3.43(\mathrm{t}, J=$ $4.7 \mathrm{~Hz}, 2 \mathrm{H}), 2.48(\mathrm{q}, J=7.4 \mathrm{~Hz}, 2 \mathrm{H}), 2.27(\mathrm{t}, J=7.6 \mathrm{~Hz}, 2 \mathrm{H}), 1.57(\mathrm{~h}, J=7.4,6.8 \mathrm{~Hz}, 4 \mathrm{H}), 1.36$ - $1.20(\mathrm{~m}, 13 \mathrm{H}) .{ }^{13} \mathbf{C}$ NMR $(75 \mathrm{MHz}$, Chloroform- $d) \delta 171.67,66.96,66.69,46.09,41.90$, 34.10, 33.20, 29.52, 29.47, 29.11, 28.43, 25.32, 24.75. HRMS (ESI) $m / z:[\mathrm{M}+\mathrm{H}]$ calculated for $\mathrm{C}_{15} \mathrm{H}_{30} \mathrm{NO}_{2} \mathrm{~S} 288.1997$, found 288.1990.

\section{N-phenylbenzamide (3ta):}

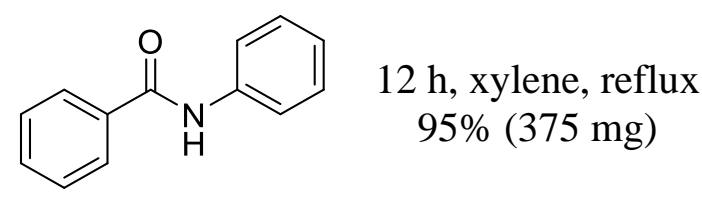

White solid, Melting point: $163-165^{\circ} \mathrm{C}$ (Meltemp),

$\mathrm{Rf}=0.57$ in hexanes:ethyl acetate 50:50 (UV, $\left.\mathrm{I}_{2}\right)$

${ }^{1}$ H NMR (300 MHz, Chloroform- $d$ ) $\delta 7.96(\mathrm{~s}, 1 \mathrm{H}), 7.86(\mathrm{dd}, J=8.0,1.6 \mathrm{~Hz}, 2 \mathrm{H}), 7.69-7.61$ $(\mathrm{m}, 2 \mathrm{H}), 7.60-7.51(\mathrm{~m}, 1 \mathrm{H}), 7.47(\mathrm{dd}, J=8.2,6.9 \mathrm{~Hz}, 2 \mathrm{H}), 7.37(\mathrm{t}, J=8.0 \mathrm{~Hz}, 2 \mathrm{H}), 7.16(\mathrm{td}, J$ $=7.4,1.3 \mathrm{~Hz}, 1 \mathrm{H}) .{ }^{13} \mathbf{C}$ NMR $(75 \mathrm{MHz}$, Chloroform- $d$ ) $\delta 166.12,138.62,135.12,131.26,128.52$, 128.15, 127.46, 123.85, 120.48. HRMS (ESI) $m / z:[\mathrm{M}+\mathrm{H}]$ calculated for $\mathrm{C}_{13} \mathrm{H}_{12} \mathrm{NO} 198.0919$, found 198.0911.

Characterization is in agreement with previous reports for this compound. ${ }^{32}$ 
N-phenylnicotinamide (3to):

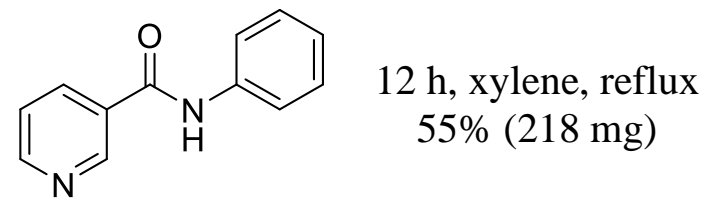

White solid, Melting point: $122-124^{\circ} \mathrm{C}$ (Meltemp),

$\mathrm{Rf}=0.10$ in hexanes:ethyl acetate 50:50 $\left(\mathrm{UV}, \mathrm{I}_{2}\right)$

${ }^{1}$ H NMR (300 MHz, Chloroform- $d$ ) $\delta 9.05(\mathrm{~d}, J=2.3 \mathrm{~Hz}, 1 \mathrm{H}), 8.69$ (dd, $\left.J=4.8,1.7 \mathrm{~Hz}, 1 \mathrm{H}\right)$, $8.55(\mathrm{~s}, 1 \mathrm{H}), 8.16(\mathrm{dt}, J=7.9,2.0 \mathrm{~Hz}, 1 \mathrm{H}), 7.62(\mathrm{dd}, J=7.7,1.6 \mathrm{~Hz}, 2 \mathrm{H}), 7.43-7.30(\mathrm{~m}, 3 \mathrm{H})$, $7.21-7.12(\mathrm{~m}, 1 \mathrm{H}) .{ }^{13} \mathbf{C}$ NMR $(75 \mathrm{MHz}$, Chloroform- $d) \delta 163.97,152.17,147.82,137.45$, 135.40, 130.79, 129.04, 124.97, 123.61, 120.55. HRMS (ESI) $m / z:[\mathrm{M}+\mathrm{H}]$ calculated for $\mathrm{C}_{12} \mathrm{H}_{11} \mathrm{~N}_{2} \mathrm{O}$ 199.0871, found 199.0859.

Characterization is in agreement with previous reports for this compound. ${ }^{33}$

\section{Characterization of mechanistic intermediates:}

\section{Ammonia-tribenzyloxyborane (4aa)}<smiles></smiles>

$1 \mathrm{~h}$, xylene, $100^{\circ} \mathrm{C}$ $\sim 99 \%(3.87 \mathrm{~g})$

White solid, Melting point: $137-143^{\circ} \mathrm{C}$ (Meltemp), $\mathrm{Rf}=0.42$ in hexanes:ethyl acetate 50:50 (UV)

${ }^{1}$ H NMR (300 MHz, DMSO- $\left.d 6\right) \delta 7.97(\mathrm{dd}, J=22.9,7.4 \mathrm{~Hz}, 6 \mathrm{H}), 7.49(\mathrm{td}, J=20.9,17.7,7.6$ $\mathrm{Hz}, 9 \mathrm{H}), 6.47$ (s, 3H). ${ }^{13} \mathrm{C}$ NMR (75 MHz, DMSO- $\left.d 6\right) \delta 168.46,165.7,132.8,131.7,129.8$, 129.4, 128.6, 128.4. ${ }^{11}$ B NMR (96 MHz, DMSO-d6) $\delta 1.02$ (s).

\section{Dimethylamine-tripivaloxyborane (4cd)}<smiles>CN(C)[B-](OC(=O)C(C)(C)C)(OC(=O)C(C)(C)C)OC(=O)C(C)(C)C</smiles>

$4 \mathrm{~h}$, xylene, $100{ }^{\circ} \mathrm{C}$

$\sim 98 \%(3.50 \mathrm{~g})$

White solid, Melting point: $79-82^{\circ} \mathrm{C}$ (Meltemp),

$\mathrm{Rf}=0.52$ in hexanes:ethyl acetate 50:50 (Ceric Ammonium Molybdate)

${ }^{1}$ H NMR (300 MHz, DMSO-d6) $\delta 2.42$ (s, 6H), 2.36 (s, 1H), 1.16- 0.96 (m, 27H). ${ }^{13}$ C NMR (75 MHz, DMSO-d6) $\delta 180.7,176.8,38.4,37.1,34.9,28.0,27.5 .{ }^{11} \mathbf{B}$ NMR (96 MHz, Chloroform- $d$ ) $\delta 0.65$ (s). 
Benzamide-dibenzyloxyboric anhydride (complex V)

(Compound Identity is tentatively assigned)

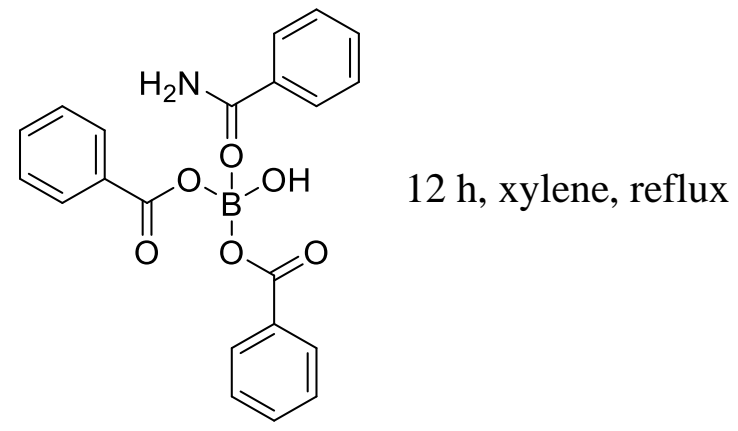

White solid

$\mathrm{Rf}=$ decomp. in hexanes:ethyl acetate 50:50 $\left(\mathrm{UV}, \mathrm{I}_{2}\right)$

${ }^{1}$ H NMR (400 MHz, DMSO-d6) $\delta 7.95(\mathrm{~s}, 1 \mathrm{H}), 7.95-7.90(\mathrm{~m}, 2 \mathrm{H}), 7.91-7.79(\mathrm{~m}, 3 \mathrm{H}), 7.63-$ $7.55(\mathrm{~m}, 1 \mathrm{H}), 7.55-7.39(\mathrm{~m}, 6 \mathrm{H}), 7.34(\mathrm{~s}, 1 \mathrm{H}) .{ }^{13} \mathrm{C}$ NMR (101 MHz, DMSO-d6) $\delta$ 168.2, 167.7, 134.6, 133.2, 131.6, 131.1, 129.6, 128.9, 128.5, 127.8. ${ }^{11} \mathbf{B}$ NMR (96 MHz, Chloroform- $d$ ) $\delta 1.86$. 


\section{References:}

1 Brown, H. C. Organic Syntheses Via Boranes, Wiley: New York, 1975, Chapter 9.

2 Ramachandran P. V.; Kulkarni A S, Int. J. Hydrogen Energy 2017, 42, 1451.

${ }^{3}$ Noth, H.; Beyer, H. Chem. Ber. 1960, 93, 931.

4 Ramachandran, P. V.; Kulkarni, A. S.; Zhao, Y.; Mei, J., Chem. Comm. 2016, 52, 11885.

5 Helten, H.; Robertson, A. P. M.; Staubitz, A.; Vance, J. R.; Haddow, M. F.; Manners, I., Chem. Eur. J. 2012, 18, 4665.

6 Jaska, C. A.; Temple, K.; Lough, A. J.; Manners, I., J. Am. Chem. Soc. 2003, 125, 9424.

7 Ortmann, R.; Schlitzer, M., Synthesis 2009, 10, 1757.

8 Zhang, C.; Li, Z.; Chen, J.; Qi, S.; Fang, Y,; Zhang, S.; Ren, C.; Lu, F.; Liang, Z.; Jiang, S.; Jia, X.; Yu, S.; Zhang, G., J. Org. Chem. 2020, 85, 7728.

9 Chen, J.; Xia, Y.; Lee, S., Org. Lett. 2020, 22, 3504.

${ }^{10}$ Chhatwal, A. R.; Lomax, H. V.; Blacker, A. J.; Williams, J. M. J.; Marce, P., Chem. Sci. 2020, 11, 5808.

${ }^{11}$ Chen, Y.; Murray, P. R. D.; Davies, A. T.; Willis, M. C., J. Am. Chem. Soc. 2018, $140,8781$.

12 Tao, Y.; Widlicka, D. W.; Hill, P. D.; Couturier, M.; Young, G. R., Org. Process Res. Dev. 2012, 16, 1805.

${ }^{13}$ Cheng, H.; Pu, M.; Kundu, G.; Schoenebeck, F., Org. Lett. 2020, 22, 331.

${ }^{14}$ Hanada, S.; Tsutsumi, E.; Motoyama, Y.; Nagashima, H., J. Am. Chem. Soc. 2009, 131, 15032.

${ }^{15}$ Guo, L.; Liu, Y.; Yao,W.;Leng, X.; Huang, Z., Org. Lett. 2013, 15, 1144.

${ }^{16}$ Peng, H.; Ma, J.; Luo, W.; Zhang, G.; Yin, B., Green Chem. 2019, 21, 2252.

${ }^{17}$ Yi, X.; Lei, S.; Liu, W.; Che, F.; Yu, C.; Liu, X.; Wang, Z.; Zhou, X.; Zhang, Y., Org. Lett. 2020, 22, 4583.

${ }^{18}$ Michiyuki, T.; Osaka, I.; Komeyama, K., Chem. Commun. 2020, 56, 1247.

${ }^{19}$ Shi, T.; Zhang, Z.; Yang, Y.; Yang, Z.;He, W.; Liu, C.; Fang, Z.; Guo, K., Tetrahedron 2020, 76, 131044.

${ }^{20}$ Alender, J.; Morgan, P.; Timberlake, J., J. Org. Chem. 1983, 48, 755.

${ }^{21}$ Irving, C. D.; Floreancig, J. T.; Laulhe, S., ACS Omega 2020, 5, 15734.

22 Torres, G. M.; Liu, Y.; Arndtsen, B. A., Science, 2020, 368, 318.

${ }^{23}$ Yoshii, T.; Tsuzuki, S.; Sakurai, S.; Sakamoto, R.; Jiang, J.; Hatanaka, M.; Matsumoto, A.; Maruoka, K., Chem. Sci. 2020, 11, 5772.

${ }^{24}$ Luecke, M.-P.; Kostenko, A.; Wang, Y.; Yao, S.; Driess, M., Angew. Chem., Int. Ed., 2019, 58, 12940.

${ }^{25}$ Sharley, D. D. S.; Williams, J. M. J., Chem. Commun. 2017, 53, 2020.

${ }^{26}$ Suchy, M.; Elmehriki, A. A. H.; Hudson, R. H. E. Org. Lett. 2011, 13, 3952.

${ }^{27}$ Ishihara, K.; Lu, Y., Chem. Sci. 2016, 7, 1276.

${ }^{28}$ Nemchik, A.; Badescu, V.; Phanstiel, O., Tetrahedron 2003, 59, 4315.

${ }^{29}$ Li, J.; Oost, R.; Maryasin, B.; Gonzalez, L.; Maulide, N., Nat. Commun. 2019, 10.

${ }^{30}$ Herszman, J. D.; Berger, M.; Waldvoel, S. R., Org. Lett. 2019, $21,7893$.

${ }^{31}$ Krause, T.; Baader, S.; Erb, B.; Gooßen, L. J., Nat. Commun. $2016,7$.

${ }^{32}$ Li, G.; Ji, C.; Hong, X.; Szostak, M., J. Am. Chem. Soc. 2019, 141, 11161.

${ }^{33}$ Li, G.; Szostak, M., Nat. Commun. 2018, 9. 
NMR spectra of ammonia-borane and amine-boranes:
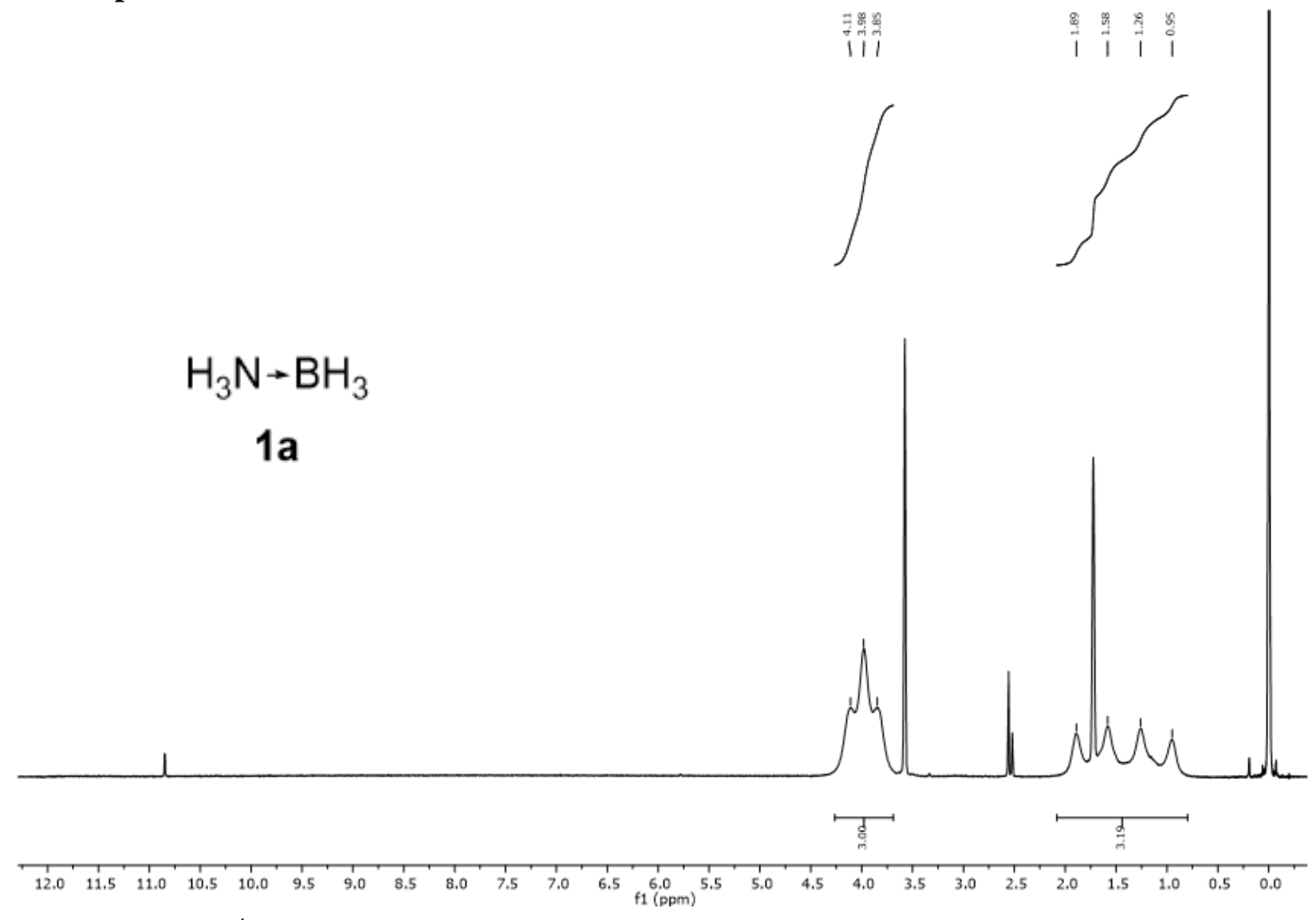

${ }^{1} \mathrm{H}$ NMR (300 MHz, Tetrahydrofuran-d8) ammonia-borane (1a)

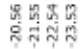

vil

$\mathrm{H}_{3} \mathrm{~N} \rightarrow \mathrm{BH}_{3}$

1a

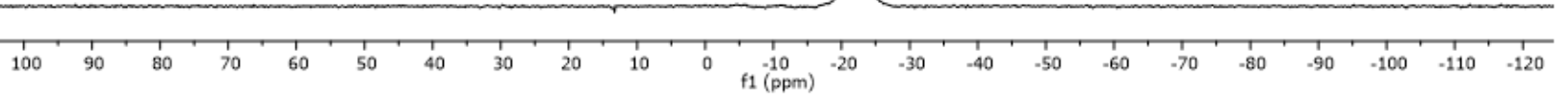

${ }^{11}$ B NMR (96 MHz, Tetrahydrofuran-d8) ammonia-borane (1a) 


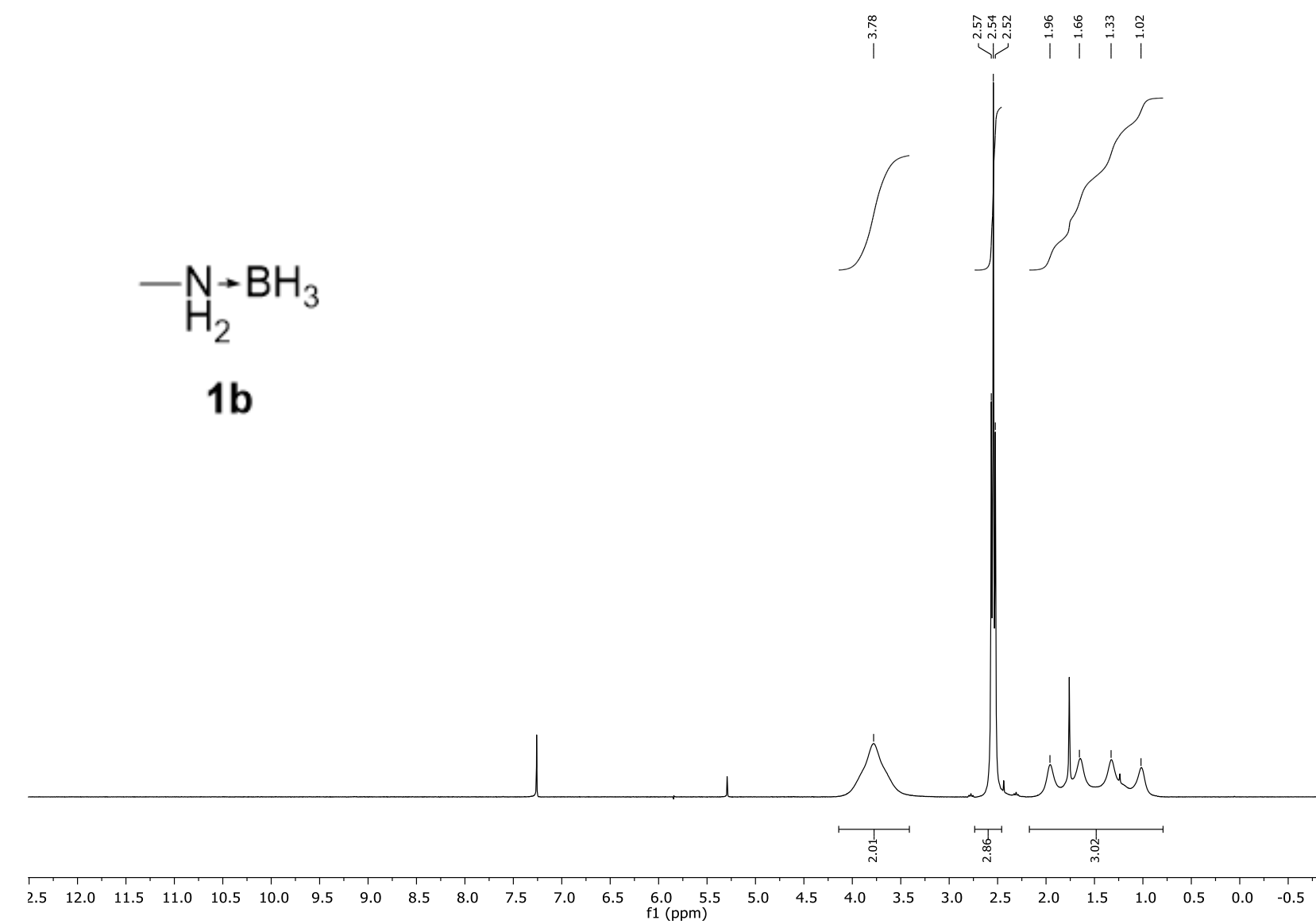

${ }^{1} \mathrm{H}$ NMR (300 MHz, Chloroform- $d$ ) methylamine-borane (1b)

$$
-\underset{\mathrm{H}}{\mathrm{H}} \rightarrow \mathrm{BH}_{2}
$$

1b

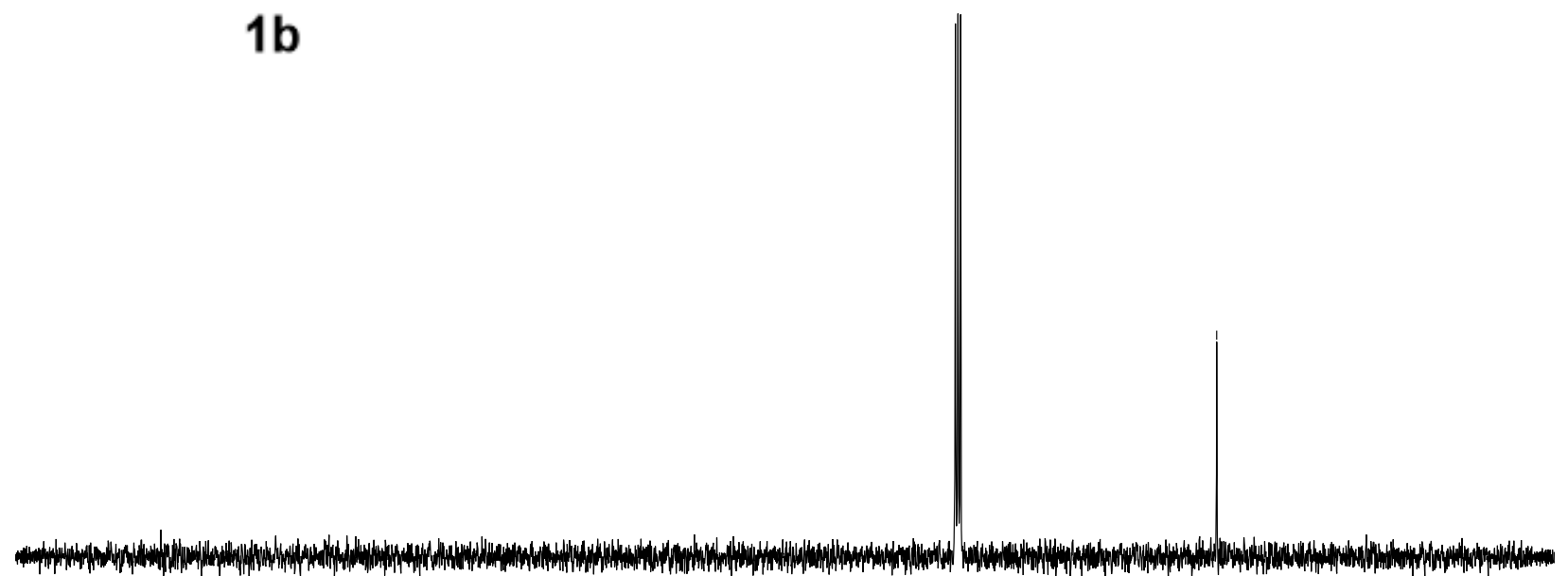

\begin{tabular}{lllllllllllllllllllllllllllllllllllll}
\hline & 230 & 220 & 210 & 200 & 190 & 180 & 170 & 160 & 150 & 140 & 130 & 120 & 110 & 100 & 90 & 80 & 70 & 60 & 50 & 40 & 30 & 20 & 10 & 0 & -10 & -2
\end{tabular}

${ }^{13} \mathrm{C}$ NMR (75 MHz, Chloroform- $d$ ) methylamine-borane (1b) 


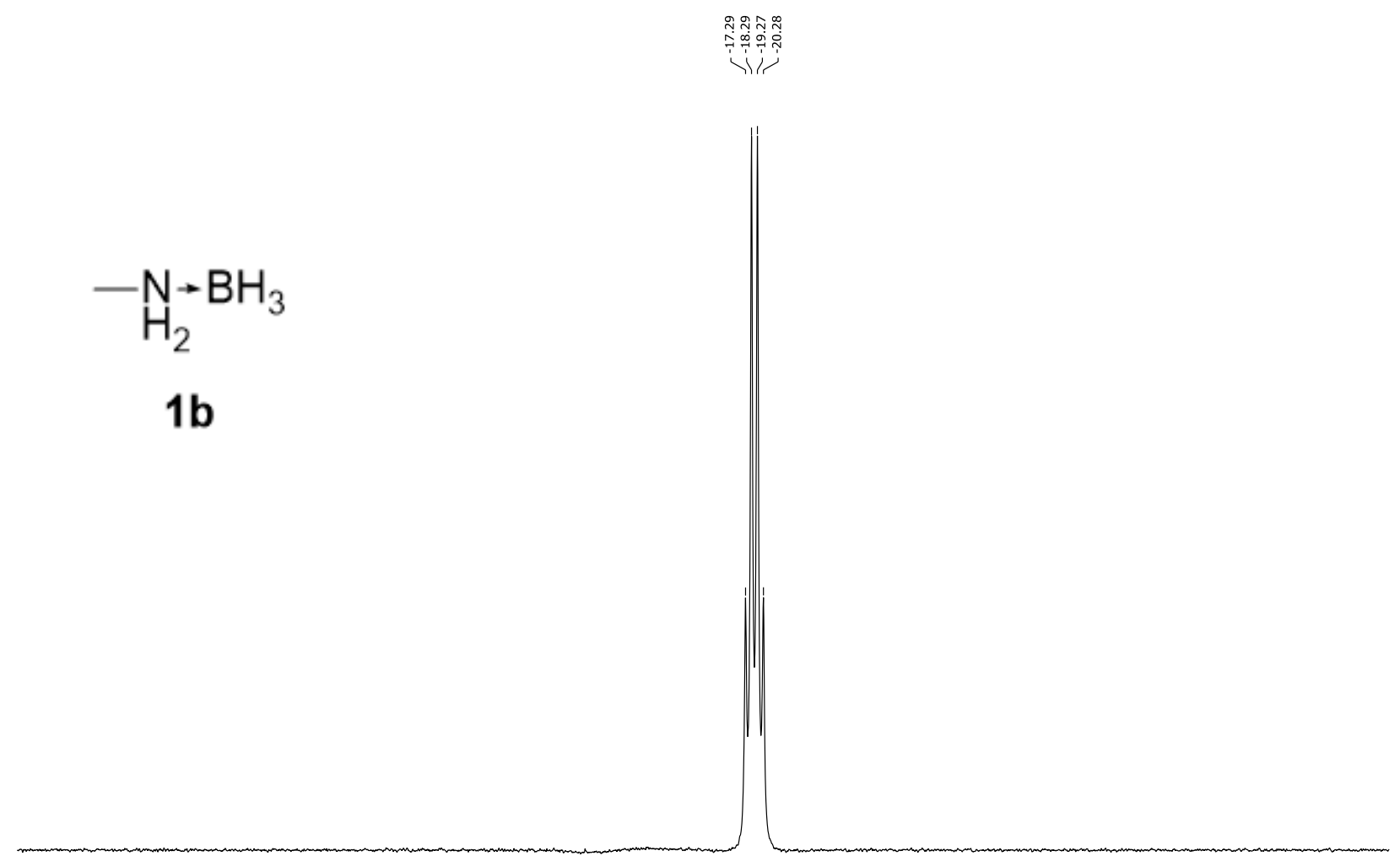

\begin{tabular}{|c|c|c|c|c|c|c|c|c|c|c|c|c|c|c|c|c|c|c|c|c|c|c|}
\hline 100 & 90 & 80 & 70 & 60 & 50 & 40 & 30 & 20 & 10 & 0 & $\begin{array}{c}-10 \\
\mathrm{f} 1(\mathrm{ppm})\end{array}$ & -20 & -30 & -40 & -50 & -60 & -70 & -80 & -90 & -100 & -110 & -120 \\
\hline
\end{tabular}

${ }^{11}$ B NMR (96 MHz, Chloroform- $d$ ) methylamine-borane (1b)

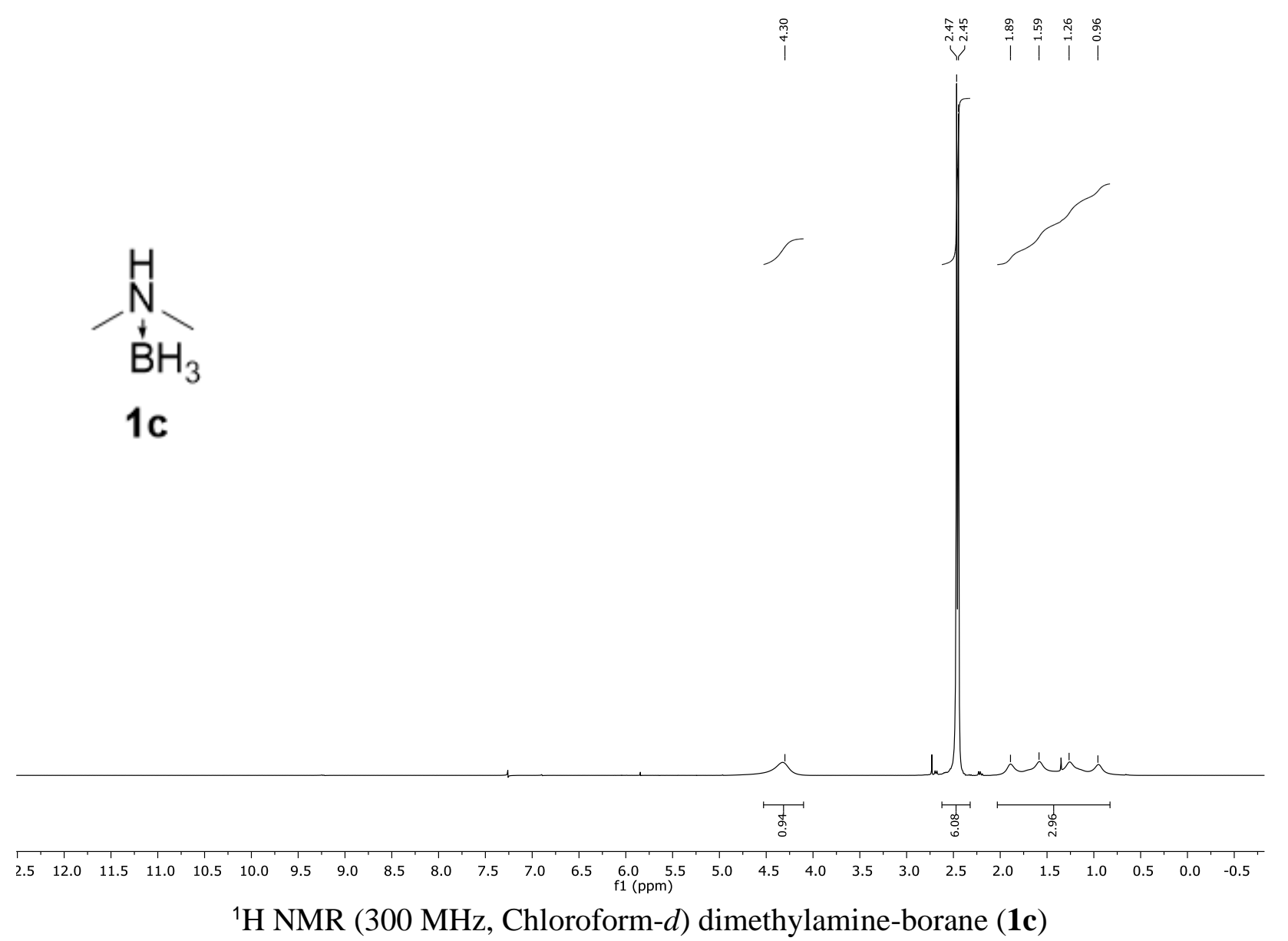



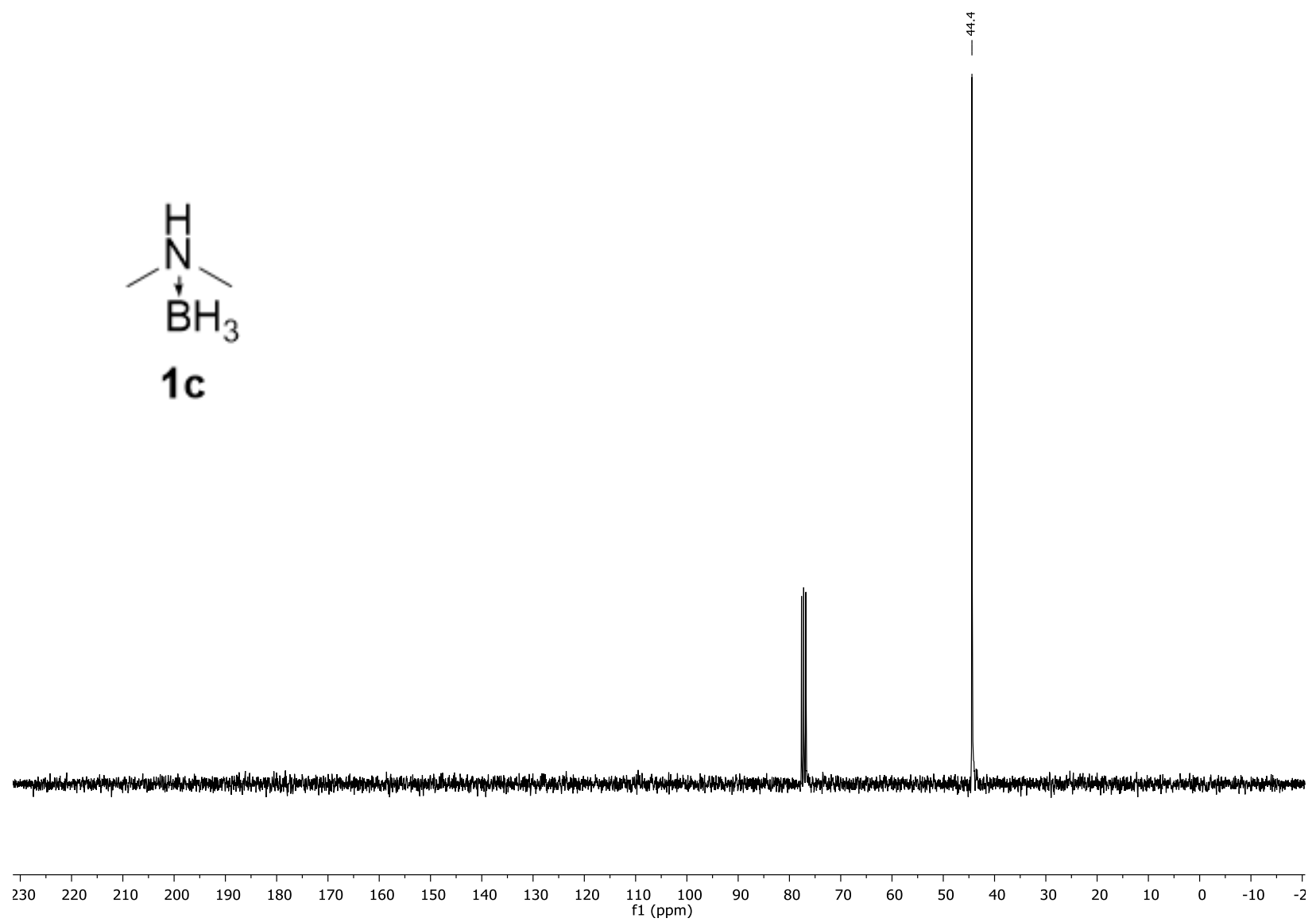

${ }^{13} \mathrm{C}$ NMR (75 MHz, Chloroform- $d$ ) dimethylamine-borane (1c)
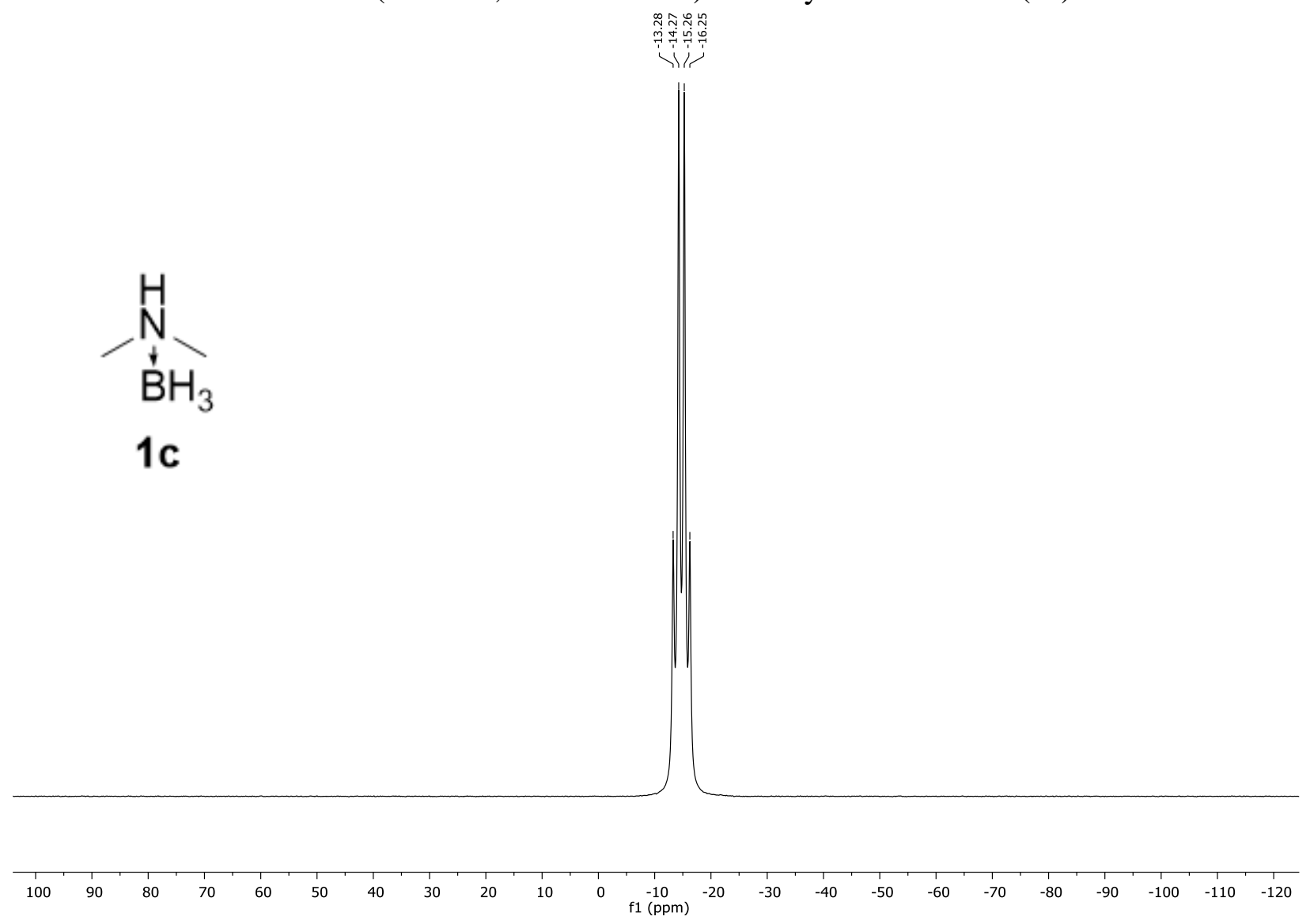

${ }^{11}$ B NMR (96 MHz, Chloroform- $d$ ) dimethylamine-borane (1c) 

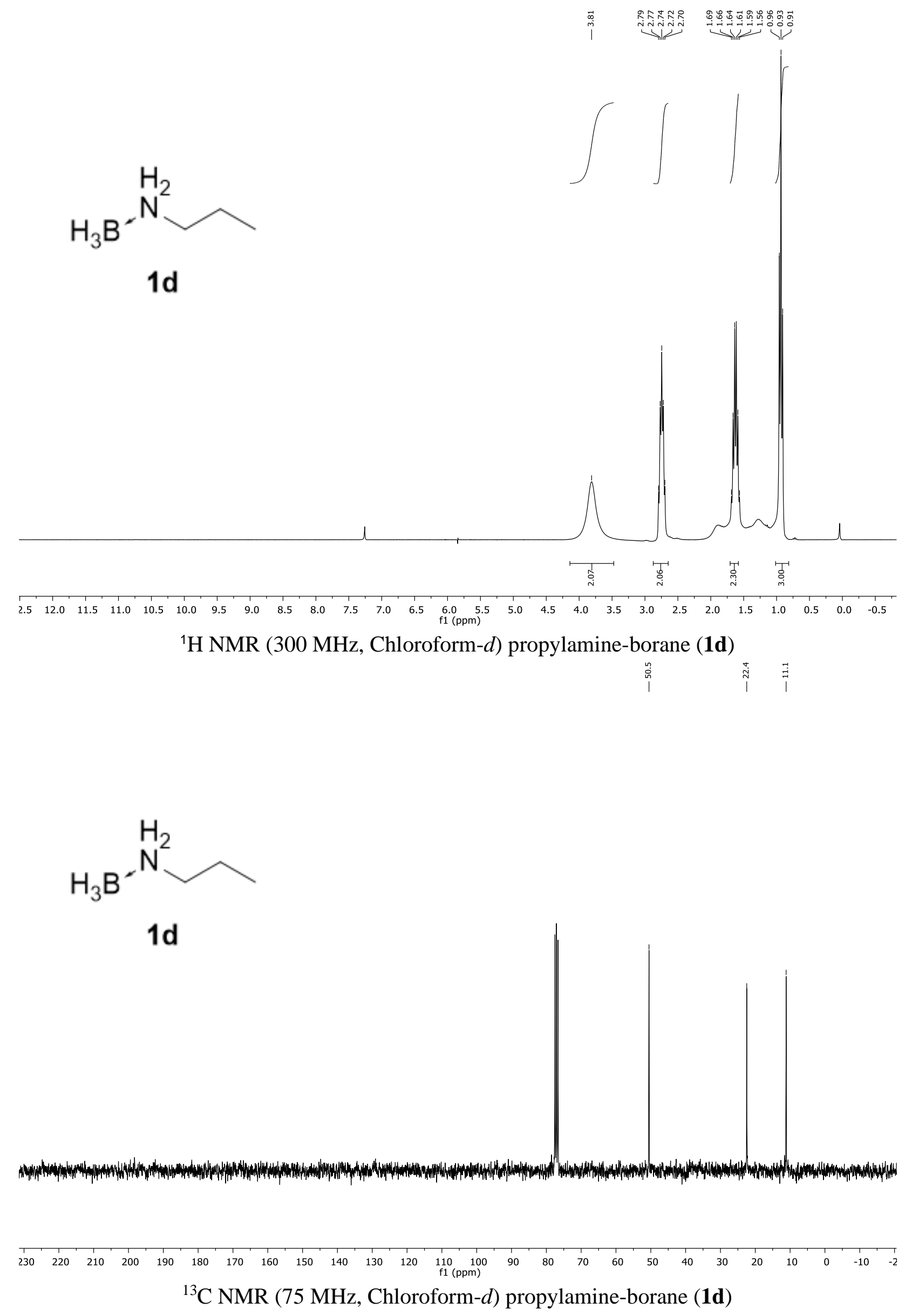

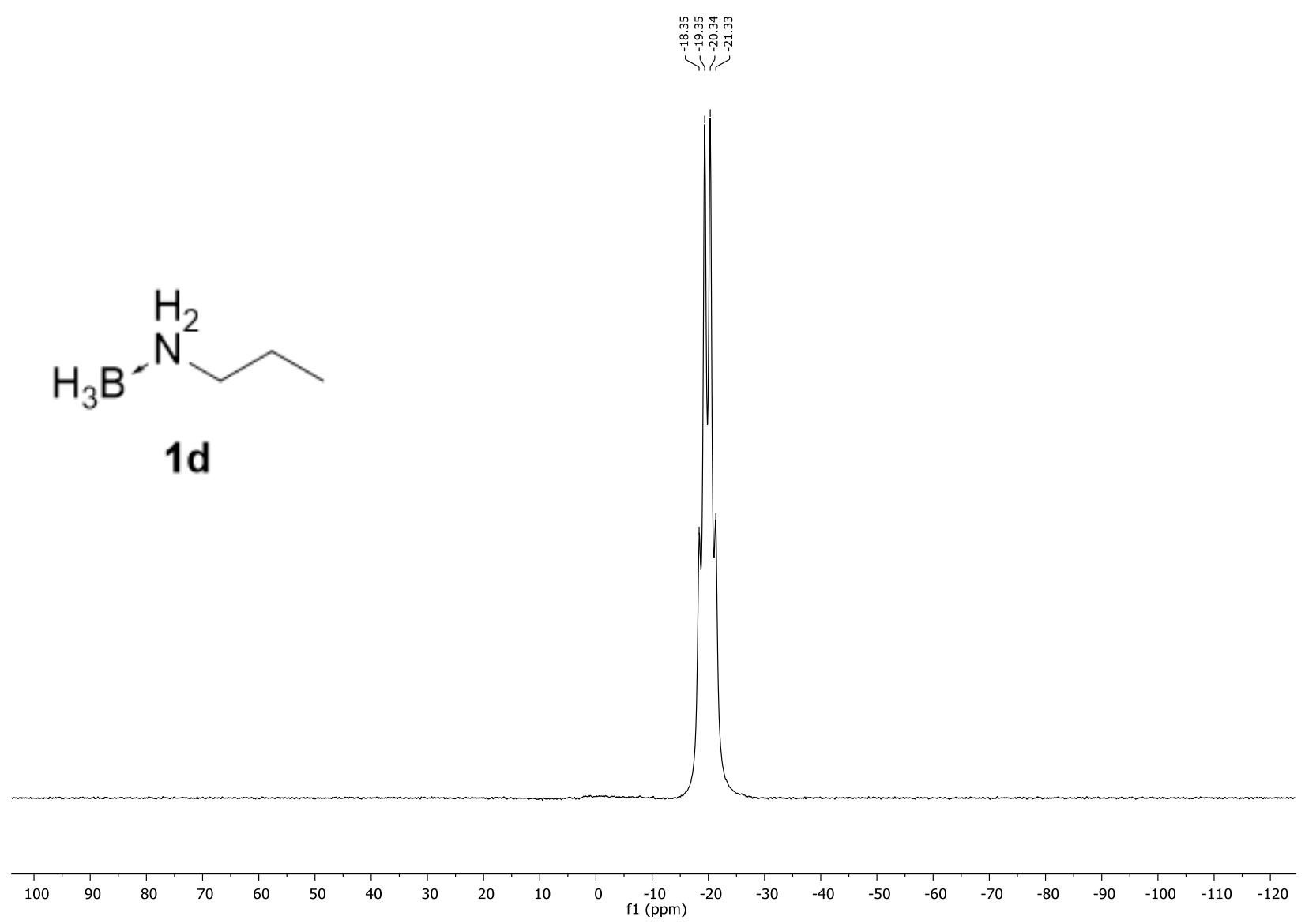

${ }^{11} \mathrm{~B}$ NMR (96 MHz, Chloroform- $d$ ) propylamine-borane (1d)
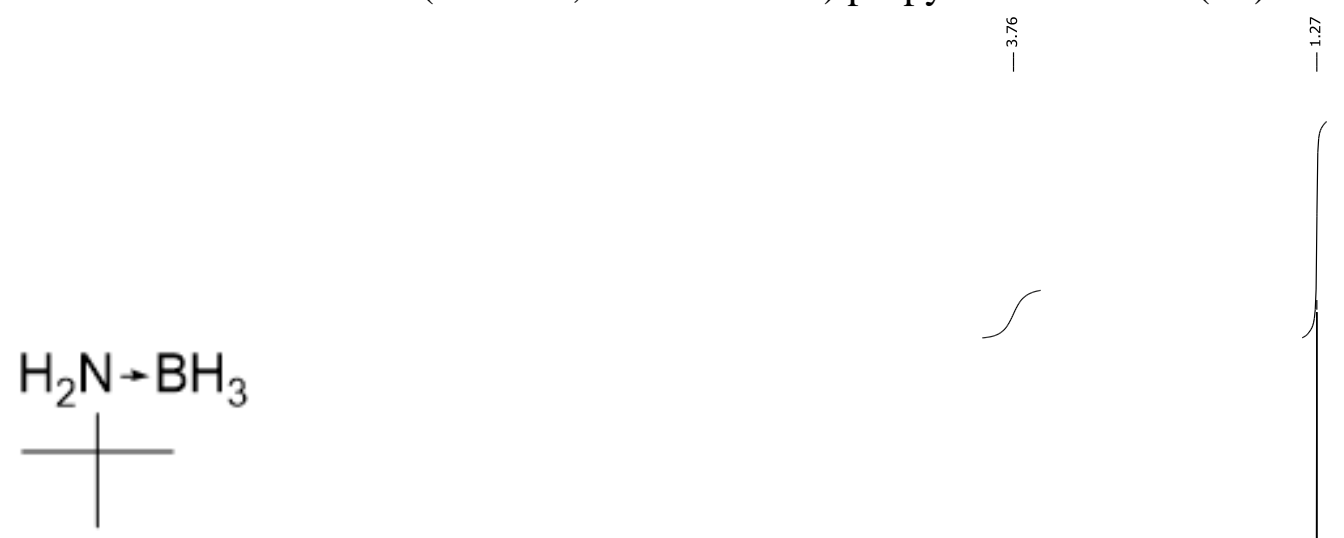

$1 \mathrm{e}$

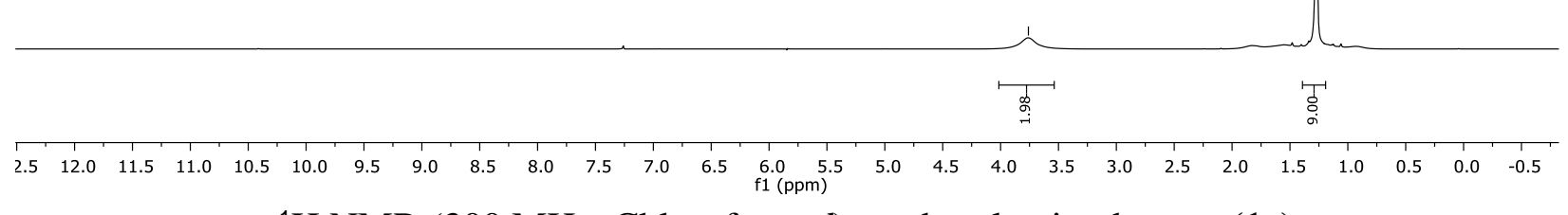

${ }^{1} \mathrm{H}$ NMR (300 MHz, Chloroform-d) tert-butylamine-borane (1e) 

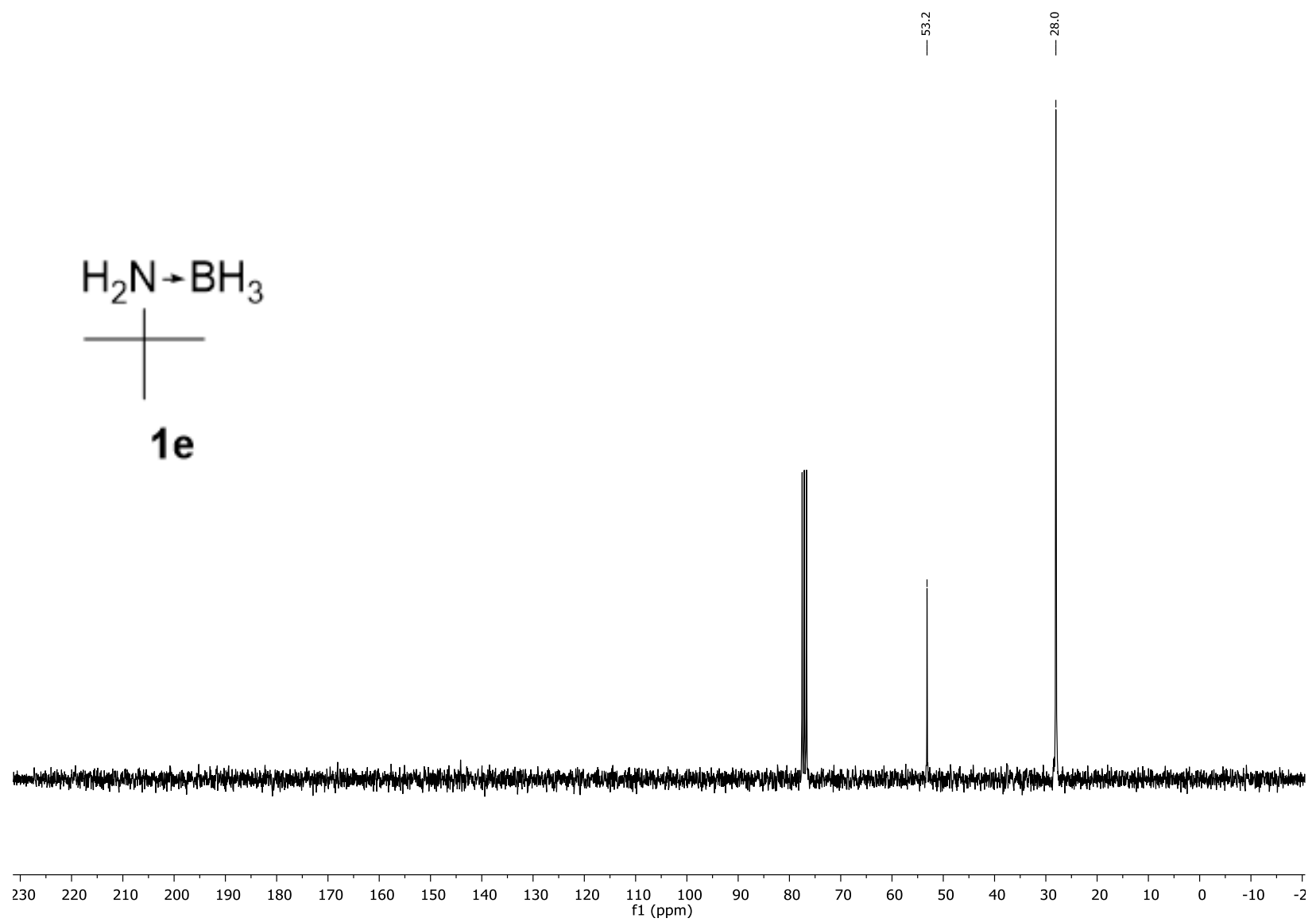

${ }^{13} \mathrm{C}$ NMR (75 MHz, Chloroform- $d$ ) tert-butylamine-borane (1e)

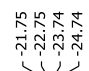

$\mathrm{H}_{2} \mathrm{~N} \rightarrow \mathrm{BH}_{3}$

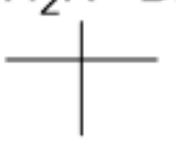

$1 \mathrm{e}$

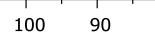

$80 \quad 70$

${ }^{11}$ B NMR (96 MHz, Chloroform- $d$ ) tert-butylamine-borane (1e) 

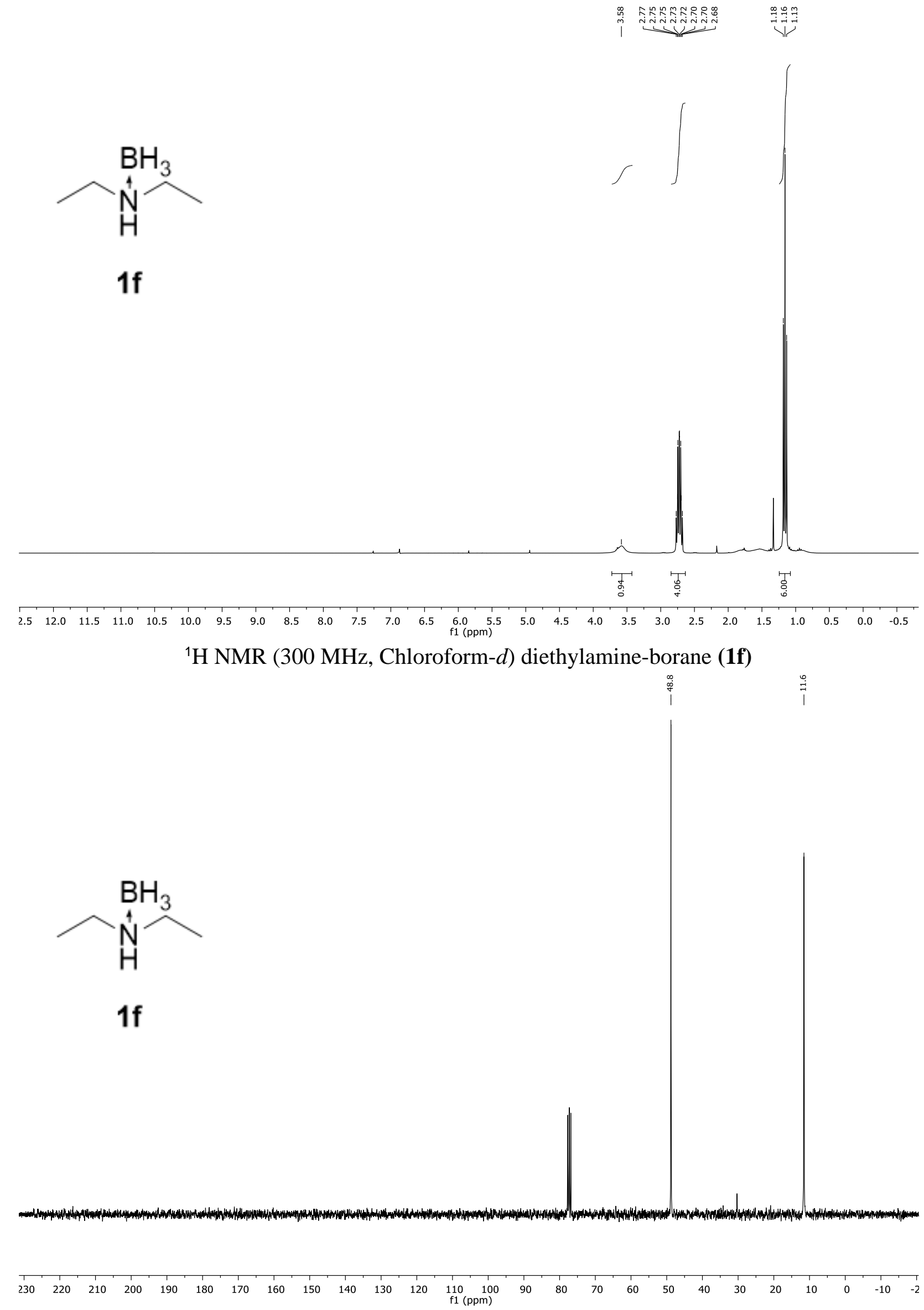

${ }^{13} \mathrm{C}$ NMR (75 MHz, Chloroform- $d$ ) diethylamine-borane (1f) 


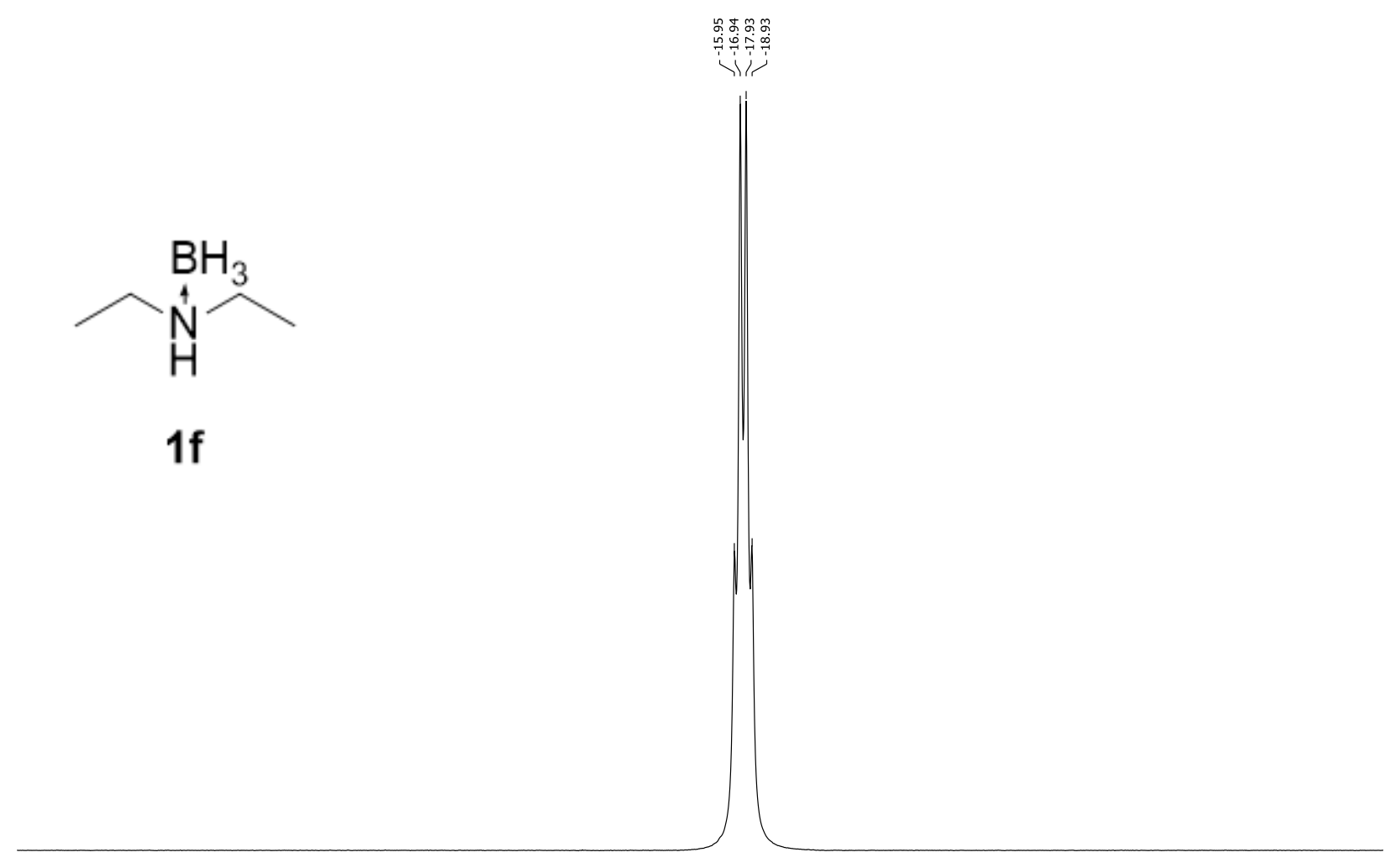

\begin{tabular}{rlllllllllllllllllllllllllll}
\hline 1 & 100 & 90 & 80 & 70 & 60 & 50 & 40 & 30 & 20 & 10 & 0 & -10 & -20 & -30 & -40 & -50 & -60 & -70 & -80 & -90 & -100 & -110 & -120
\end{tabular}

${ }^{11}$ B NMR (96 MHz, Chloroform- $d$ ) diethylamine-borane (1f)

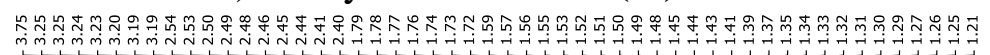
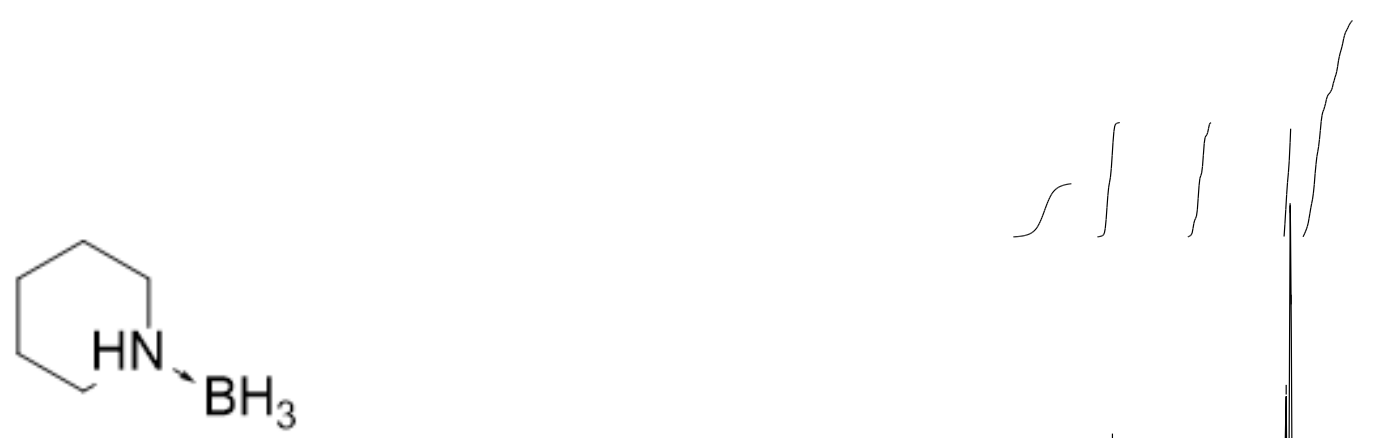

$1 \mathrm{~g}$

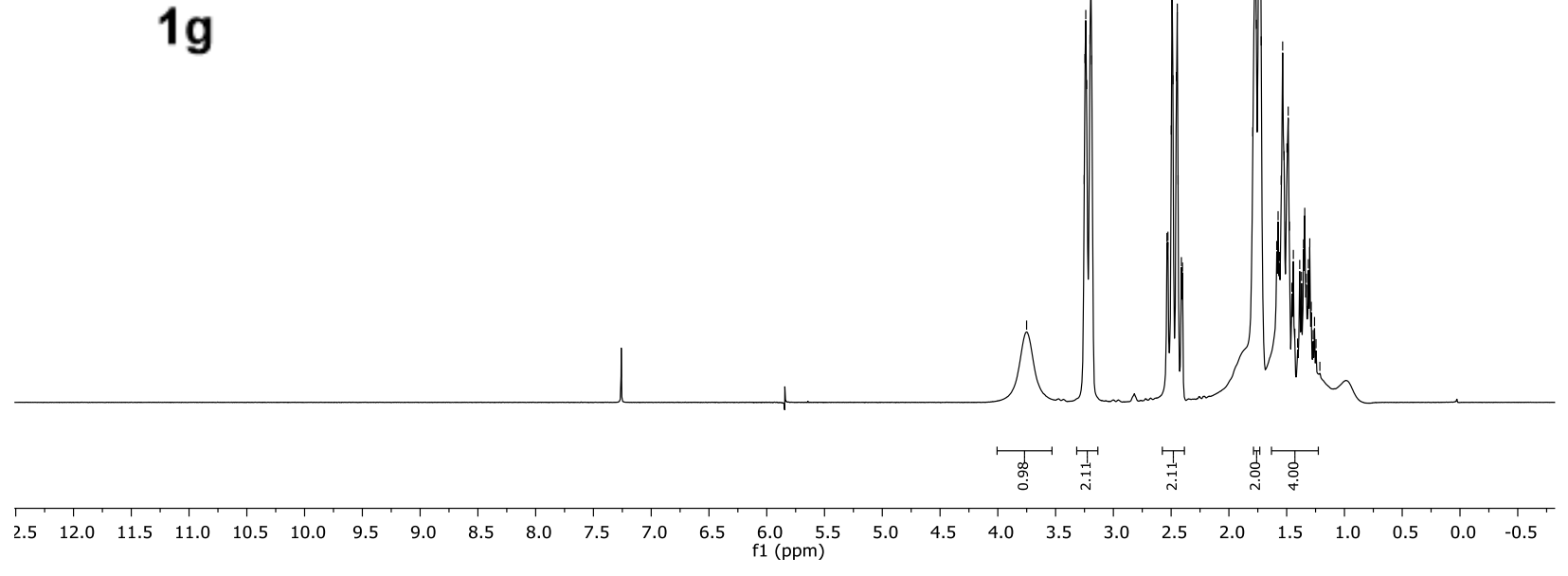

${ }^{1} \mathrm{H}$ NMR (300 MHz, Chloroform- $d$ ) piperidine-borane (1g) 


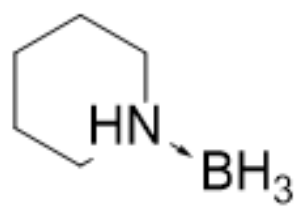

$1 \mathrm{~g}$

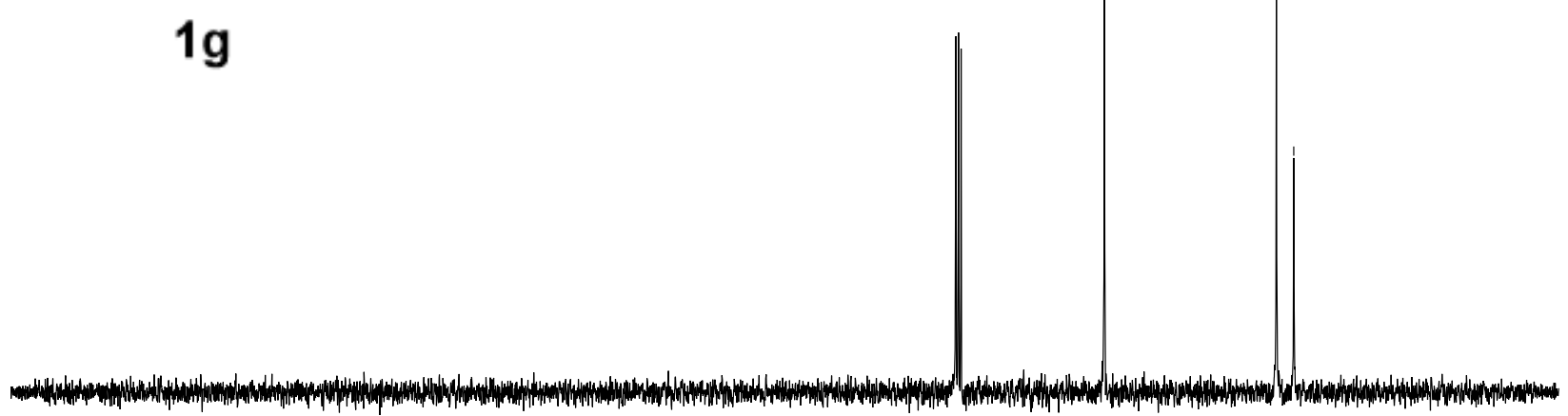

$\begin{array}{lllllllllllllllllllllllllllll}230 & 220 & 210 & 200 & 190 & 180 & 170 & 160 & 150 & 140 & 130 & 120 & \begin{array}{c}110 \\ f 1(\mathrm{ppm})\end{array} & 100 & 90 & 80 & 70 & 60 & 50 & 40 & 30 & 20 & 10 & 0 & -10 & -2\end{array}$

${ }^{13} \mathrm{C}$ NMR (75 MHz, Chloroform- $d$ ) piperidine-borane (1g)

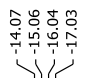

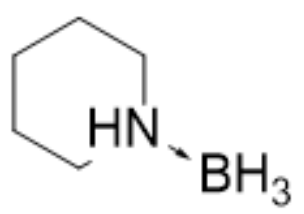

$1 \mathrm{~g}$

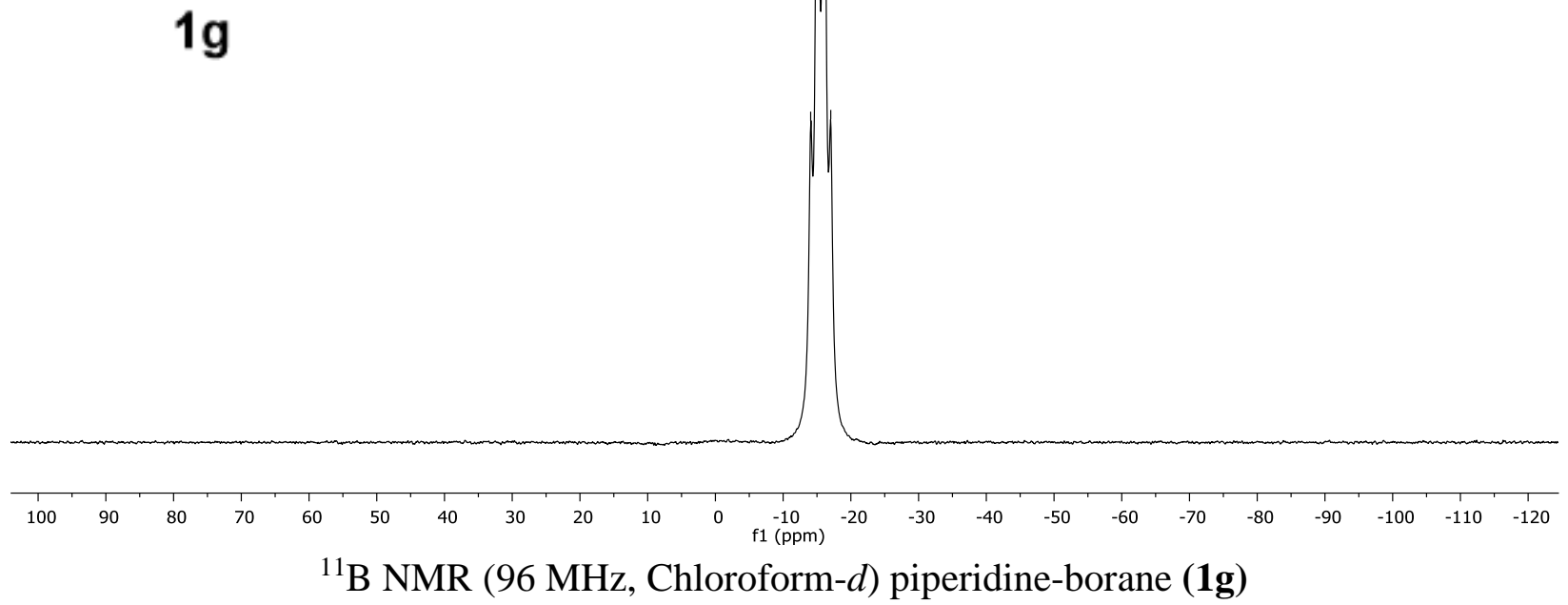




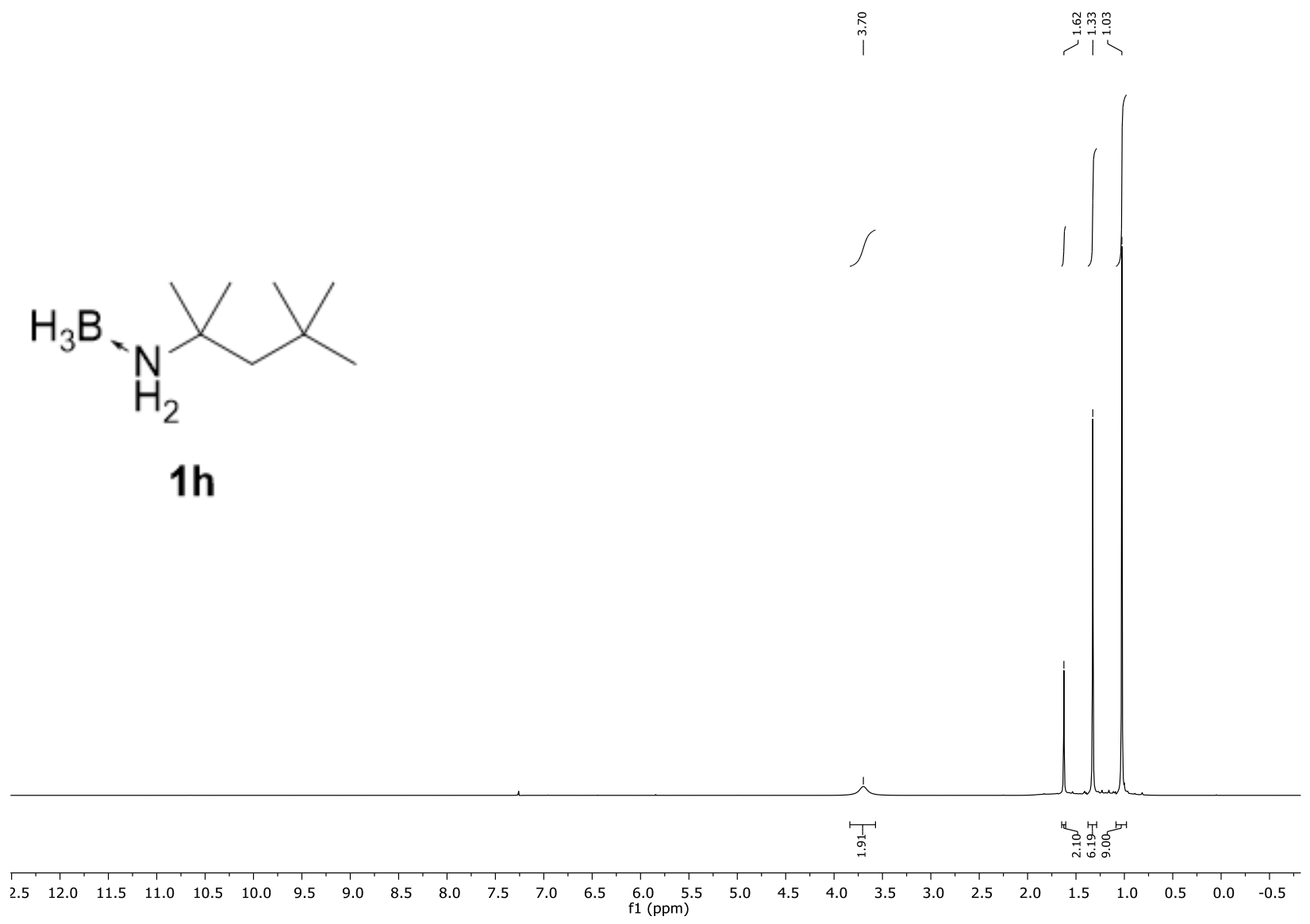

${ }^{1} \mathrm{H}$ NMR (300 MHz, Chloroform- $d$ ) tert-octylamine-borane (1h)
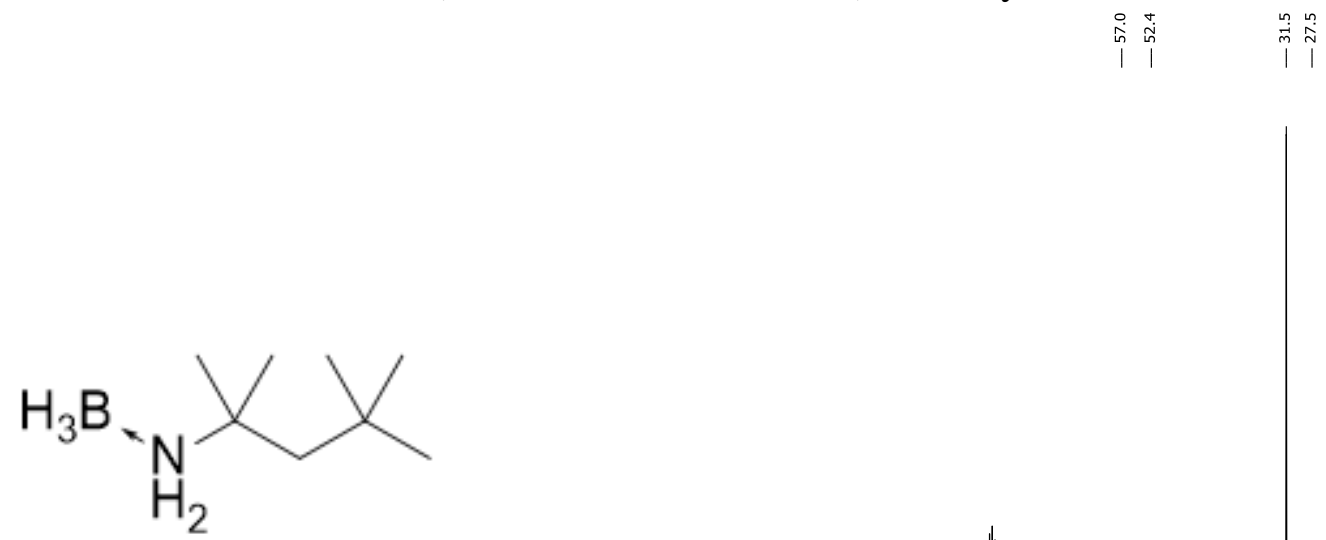

$1 \mathrm{~h}$
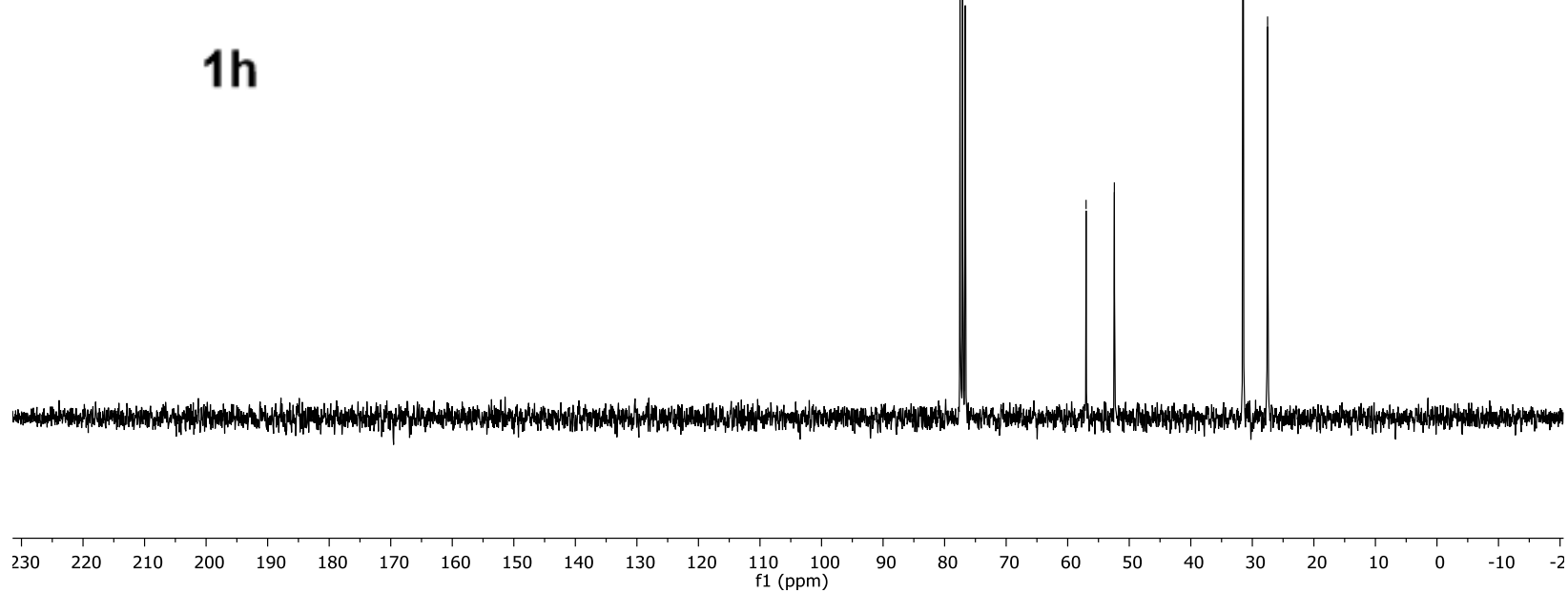

${ }^{13} \mathrm{C}$ NMR (75 MHz, Chloroform- $d$ ) tert-octylamine-borane (1h) 


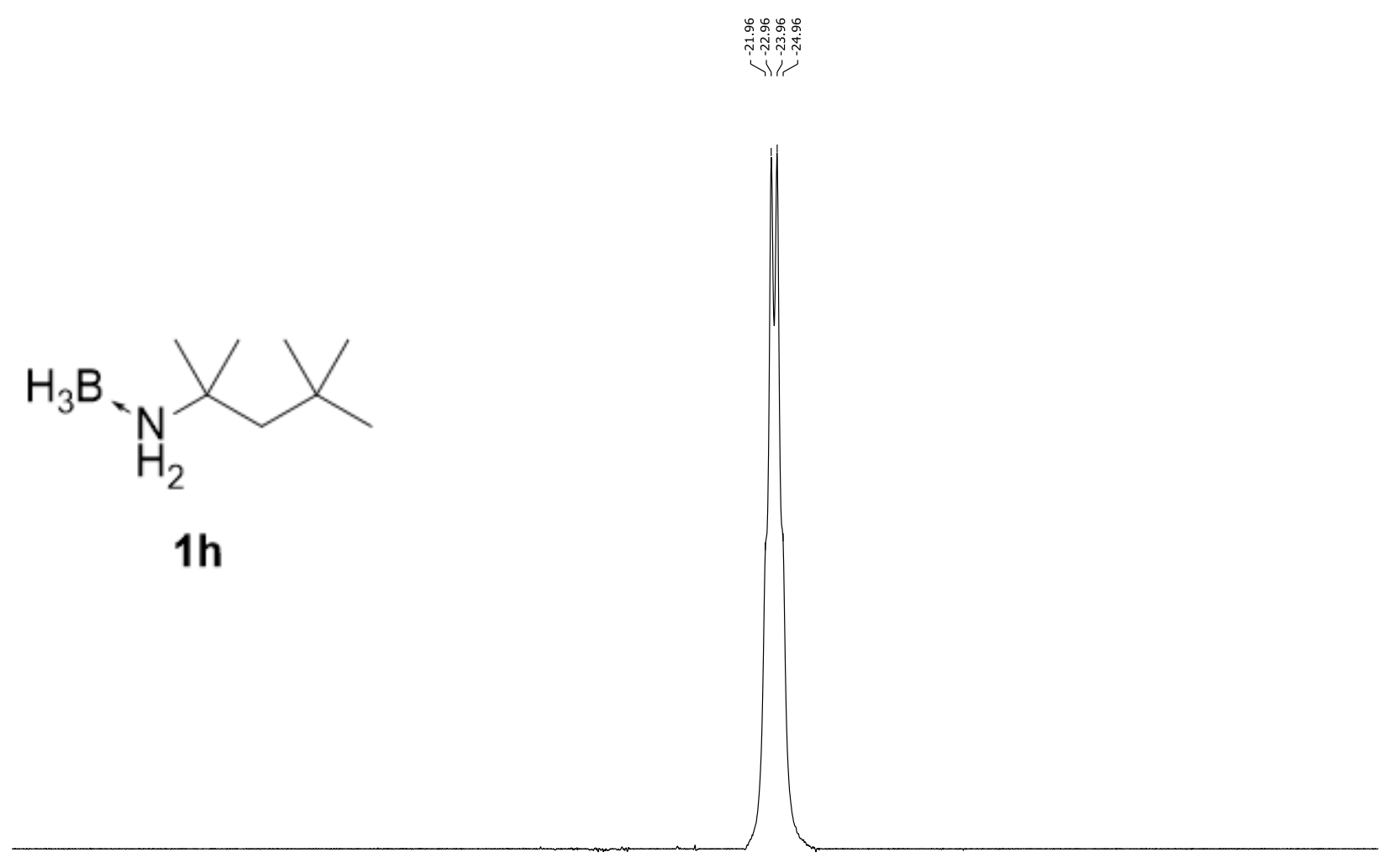

\begin{tabular}{|c|c|c|c|c|c|c|c|c|c|c|c|c|c|c|c|c|c|c|c|c|c|c|}
\hline 1 & 1 & 80 & 1 & 1 & 50 & 40 & 1 & 20 & 1 & 0 & ' & -1 & -30 & 1 & 50 & 60 & 1 & $\begin{array}{l}1 \\
-80\end{array}$ & 19 & -1 & $T_{-110}^{1}$ & $\frac{1}{-120}$ \\
\hline 100 & 90 & 80 & 70 & 60 & 50 & 40 & 30 & 20 & 10 & 0 & $\begin{array}{c}-10 \\
\mathrm{f} 1(\mathrm{ppm})\end{array}$ & -20 & -30 & -40 & -50 & -60 & -70 & -80 & -90 & -100 & -110 & -120 \\
\hline
\end{tabular}

${ }^{11}$ B NMR (96 MHz, Chloroform- $d$ ) tert-octylamine-borane (1h)

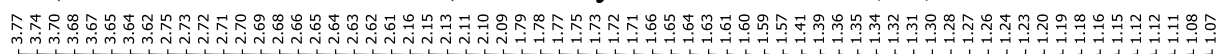

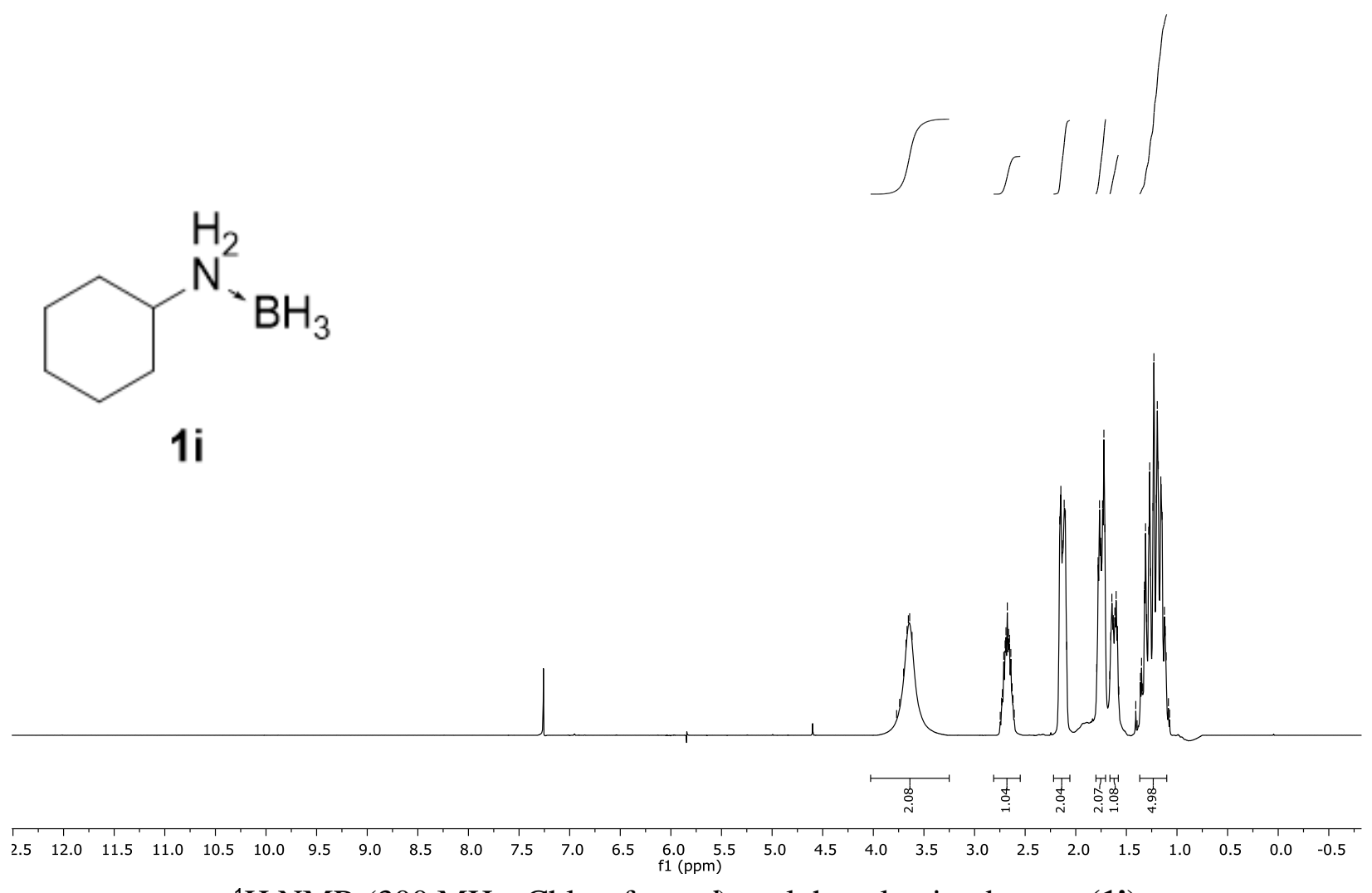

${ }^{1} \mathrm{H}$ NMR (300 MHz, Chloroform-d) cyclohexylamine-borane (1i) 


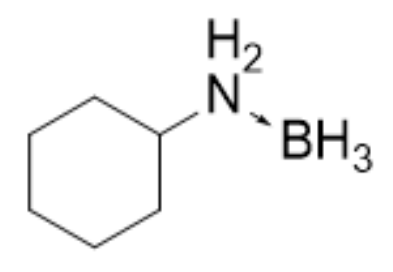

1i
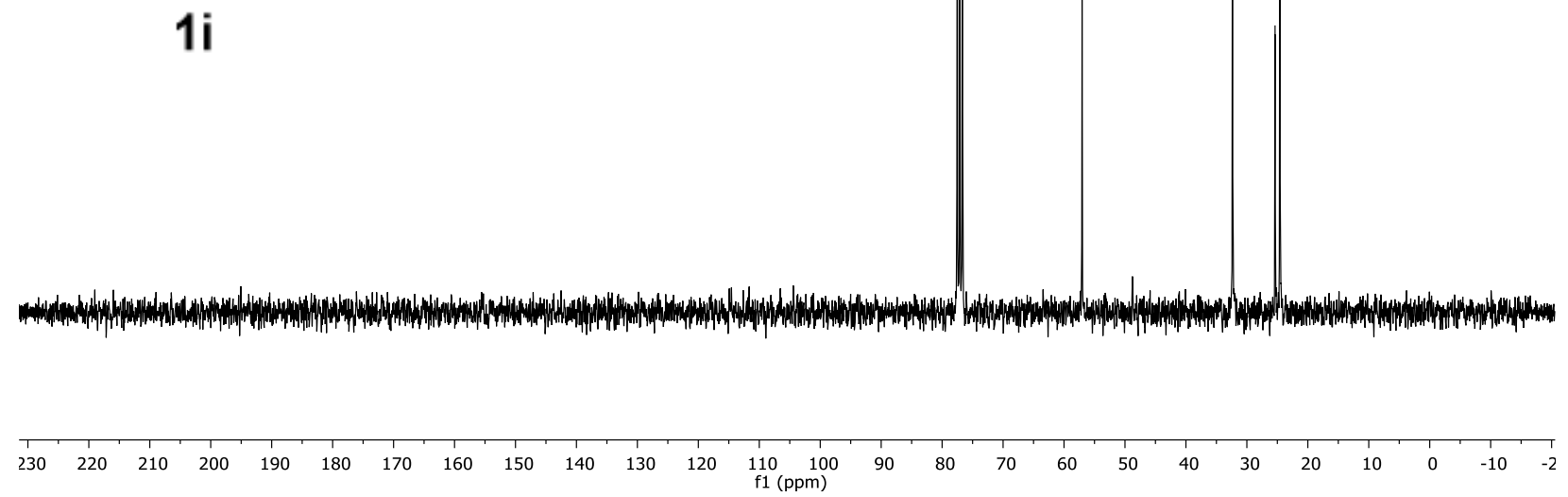

${ }^{13} \mathrm{C}$ NMR (75 MHz, Chloroform- $d$ ) cyclohexylamine-borane (1i)

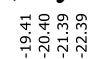

\)/

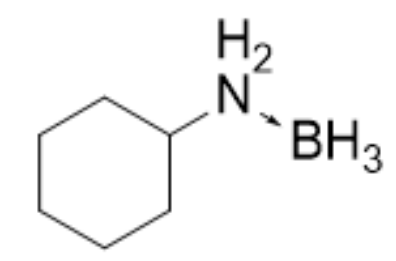

$1 \mathrm{i}$

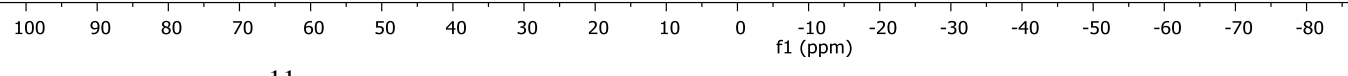

${ }^{11}$ B NMR (96 MHz, Chloroform- $d$ ) cyclohexylamine-borane (1i) 


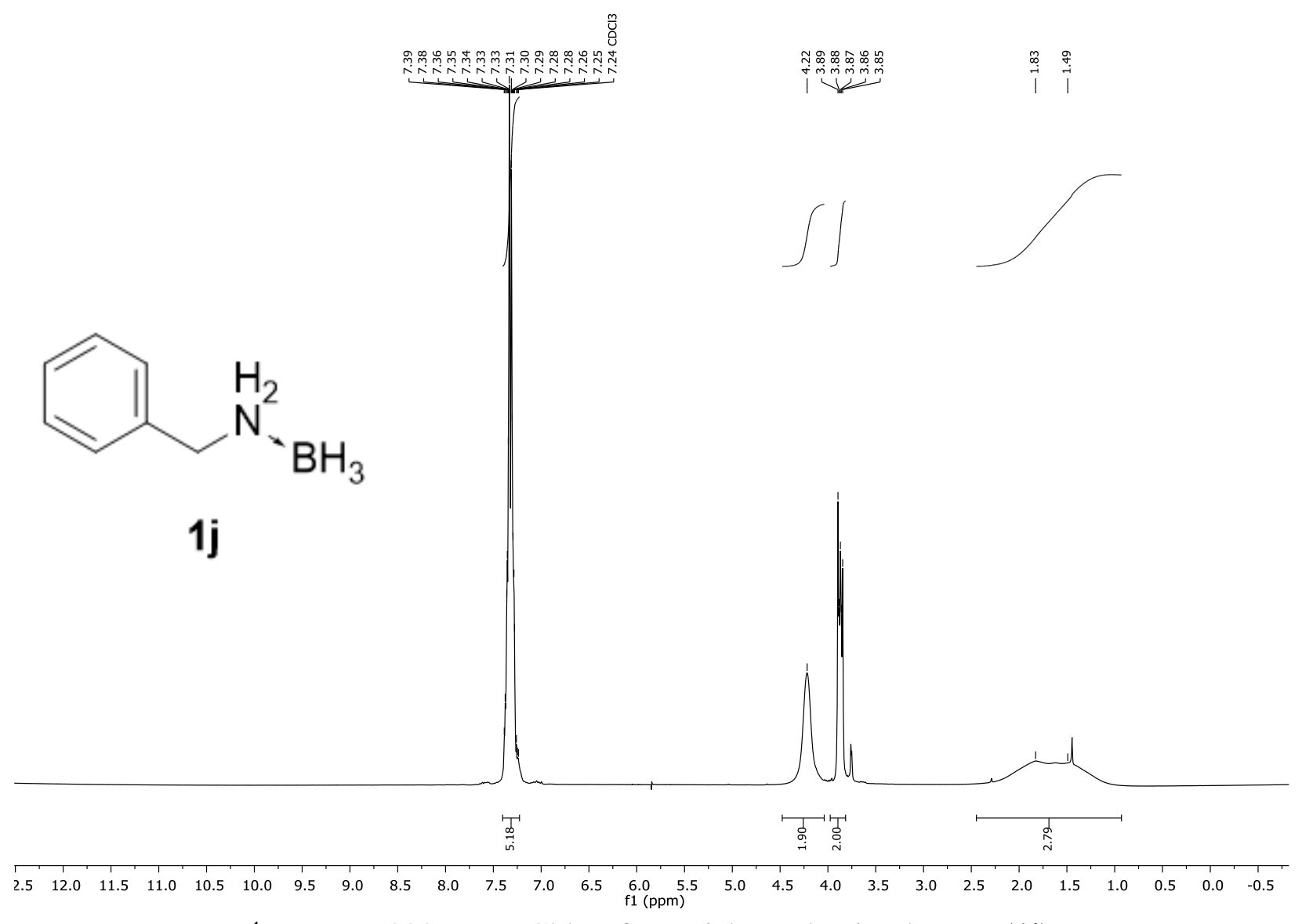

${ }^{1} \mathrm{H}$ NMR (300 MHz, Chloroform- $d$ ) benzylamine-borane (1j)

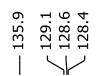

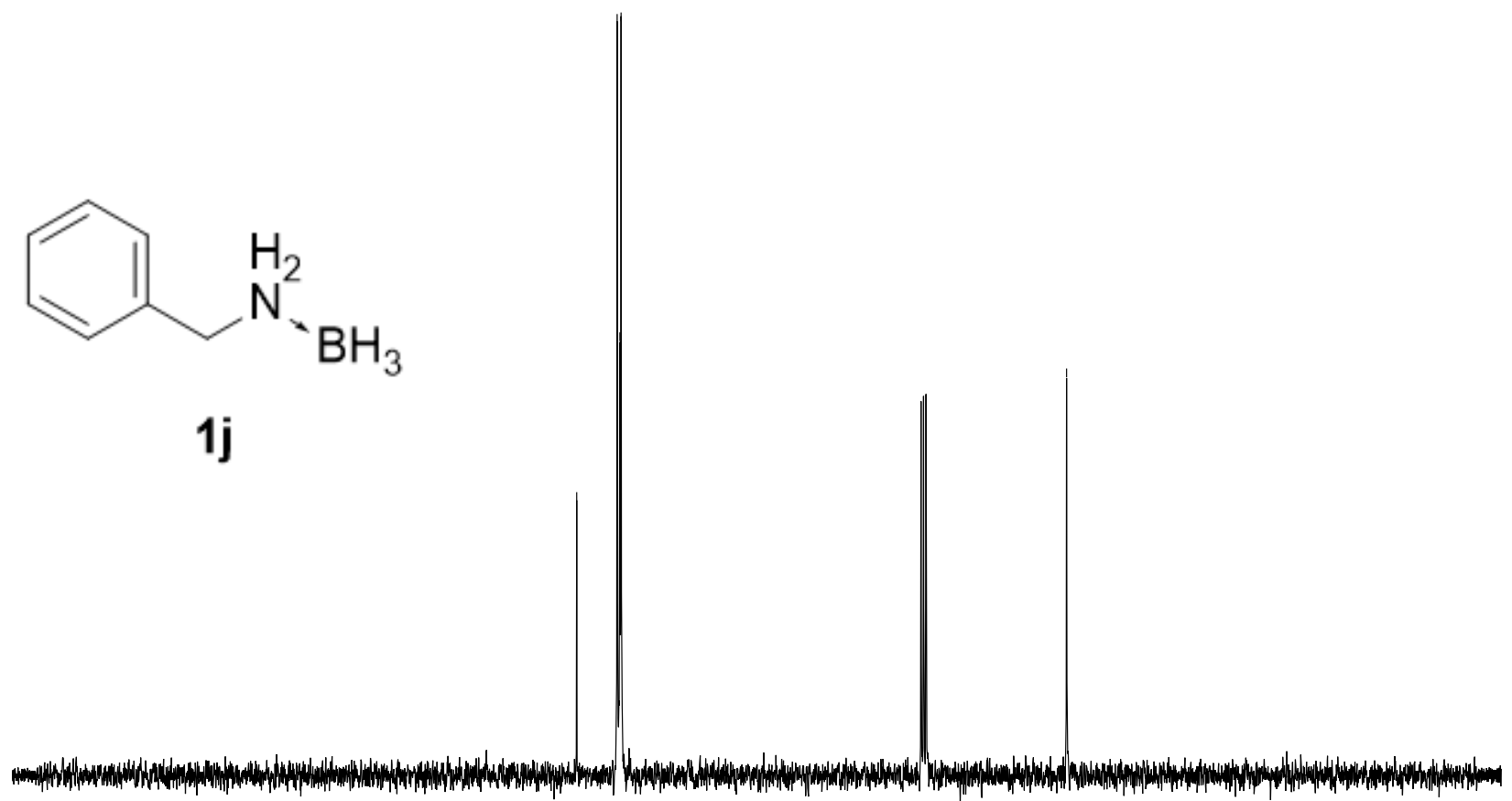

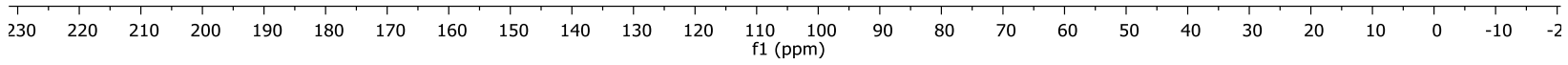

${ }^{13} \mathrm{C}$ NMR (75 MHz, Chloroform- $d$ ) benzylamine-borane (1j) 


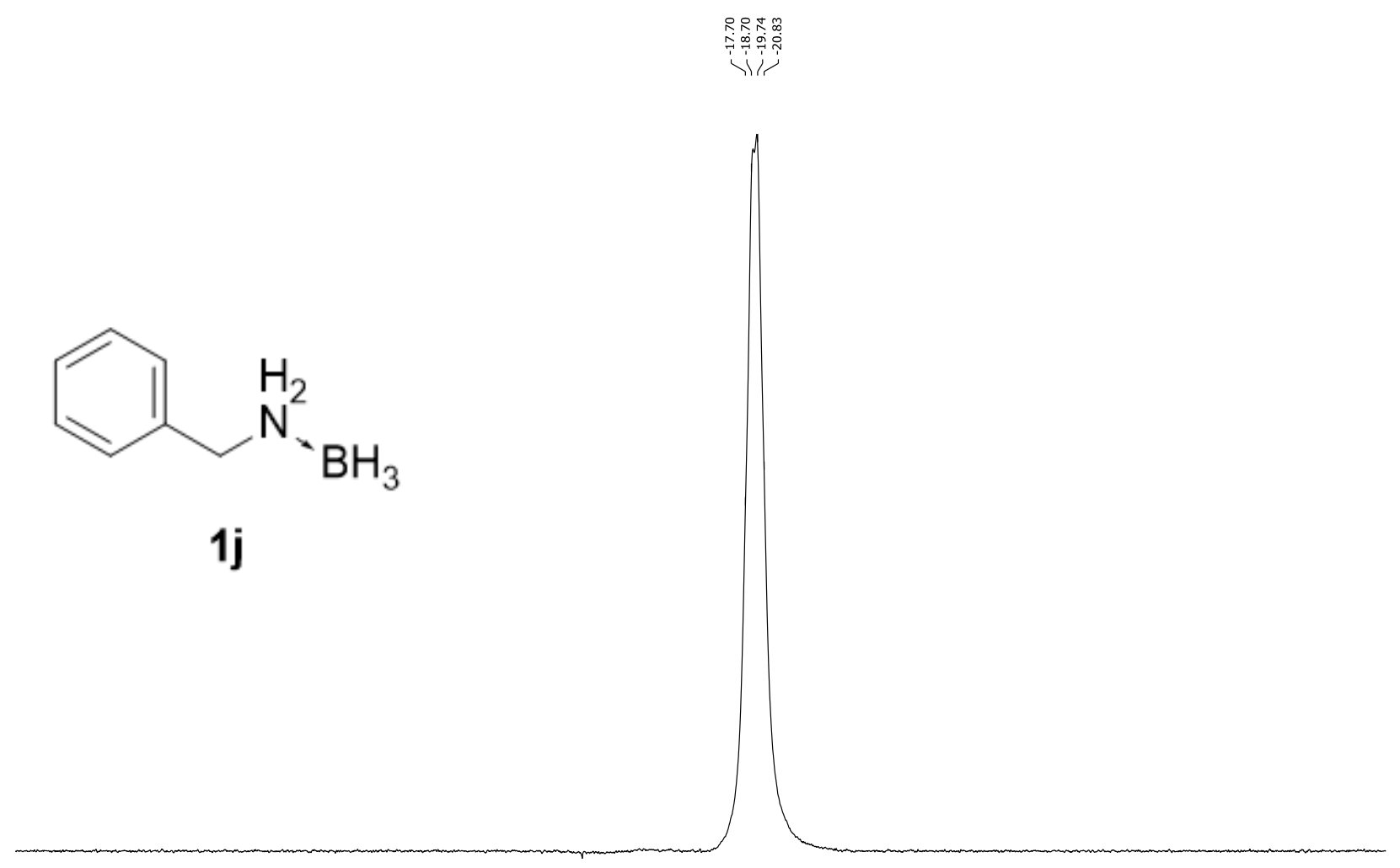

\begin{tabular}{|c|c|c|c|c|c|c|c|c|c|c|c|c|c|c|c|c|c|c|c|c|c|c|}
\hline 1 & 1 & 80 & 70 & 1 & 1 & 1 & 1 & 1 & 1 & 1 & ${ }^{1}$ & 1 & 1 & 1 & 1 & 60 & 70 & 1 & 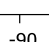 & -100 & -110 & -120 \\
\hline 100 & 90 & 80 & 70 & 60 & 50 & 40 & 30 & 20 & 10 & 0 & $\begin{array}{c}-10 \\
\mathrm{f} 1(\mathrm{ppm})\end{array}$ & -20 & -30 & -40 & -50 & -60 & -70 & -80 & -90 & -100 & -110 & -120 \\
\hline
\end{tabular}

${ }^{11}$ B NMR (96 MHz, Chloroform- $d$ ) benzylamine-borane (1j)

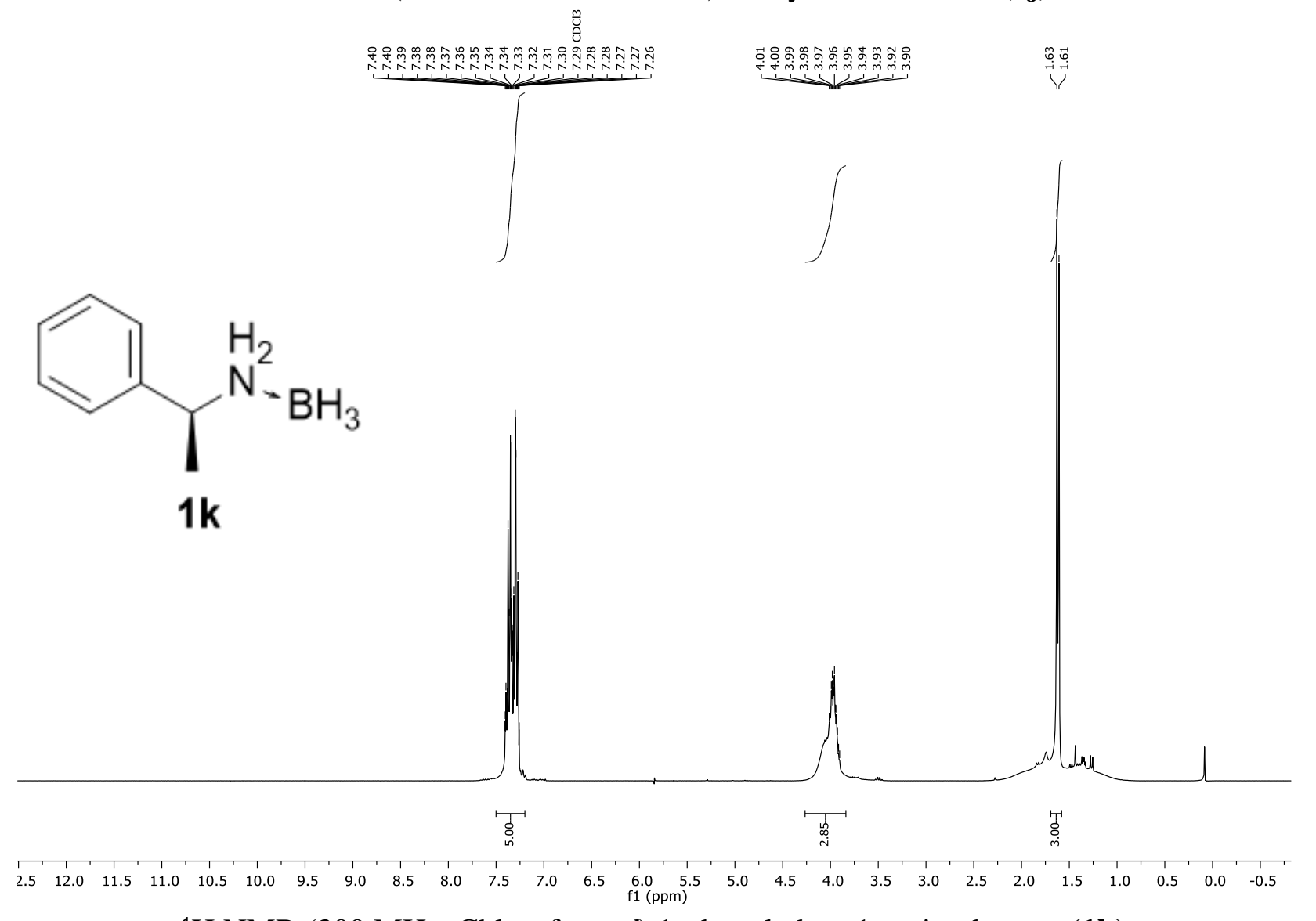

${ }^{1} \mathrm{H}$ NMR (300 MHz, Chloroform-d) 1-phenylethan-1-amine-borane (1k) 

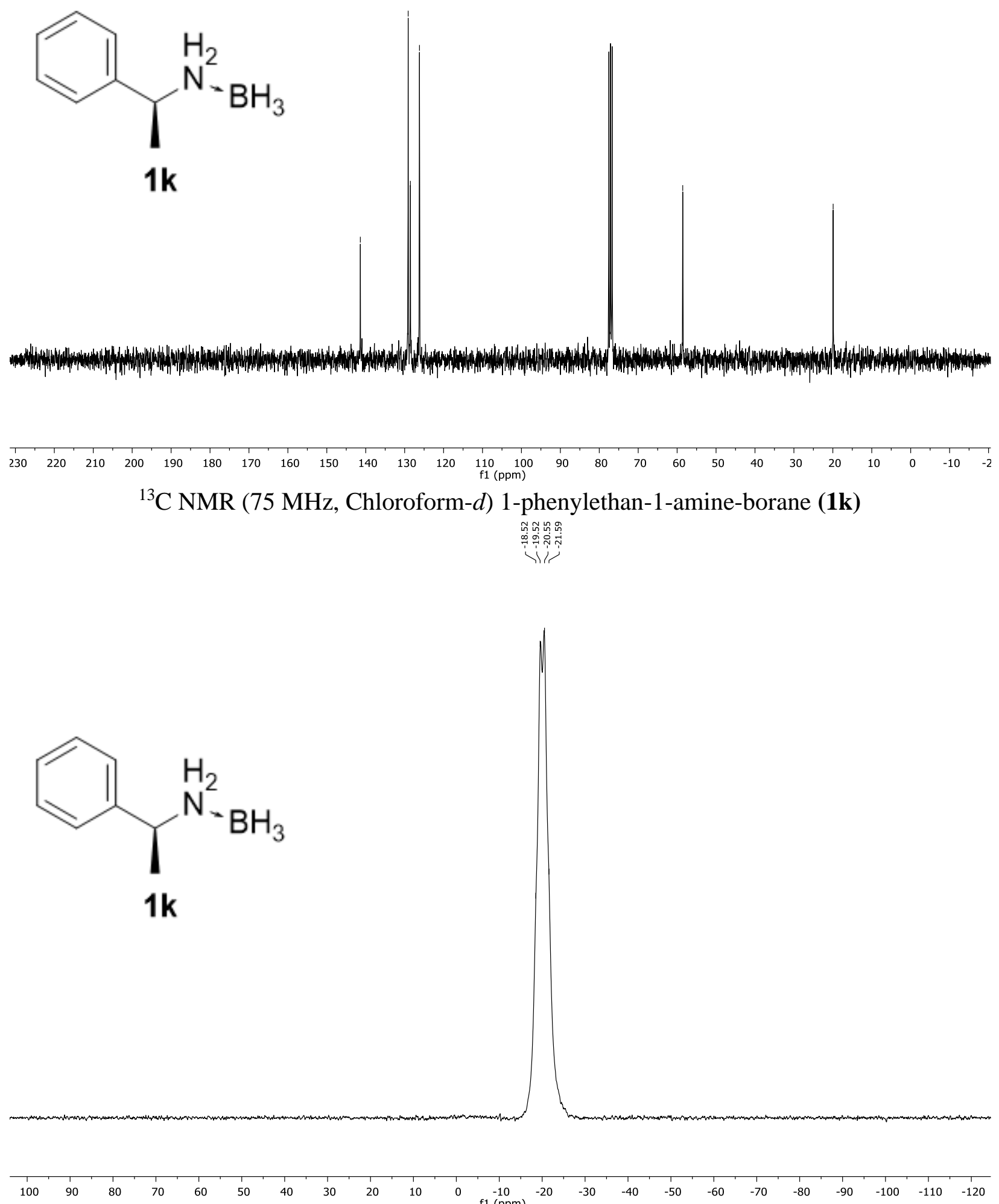

${ }^{11} \mathrm{~B}$ NMR (96 MHz, Chloroform- $d$ ) 1-phenylethan-1-amine-borane (1k) 
<smiles>[B]NCC(F)(F)F</smiles>

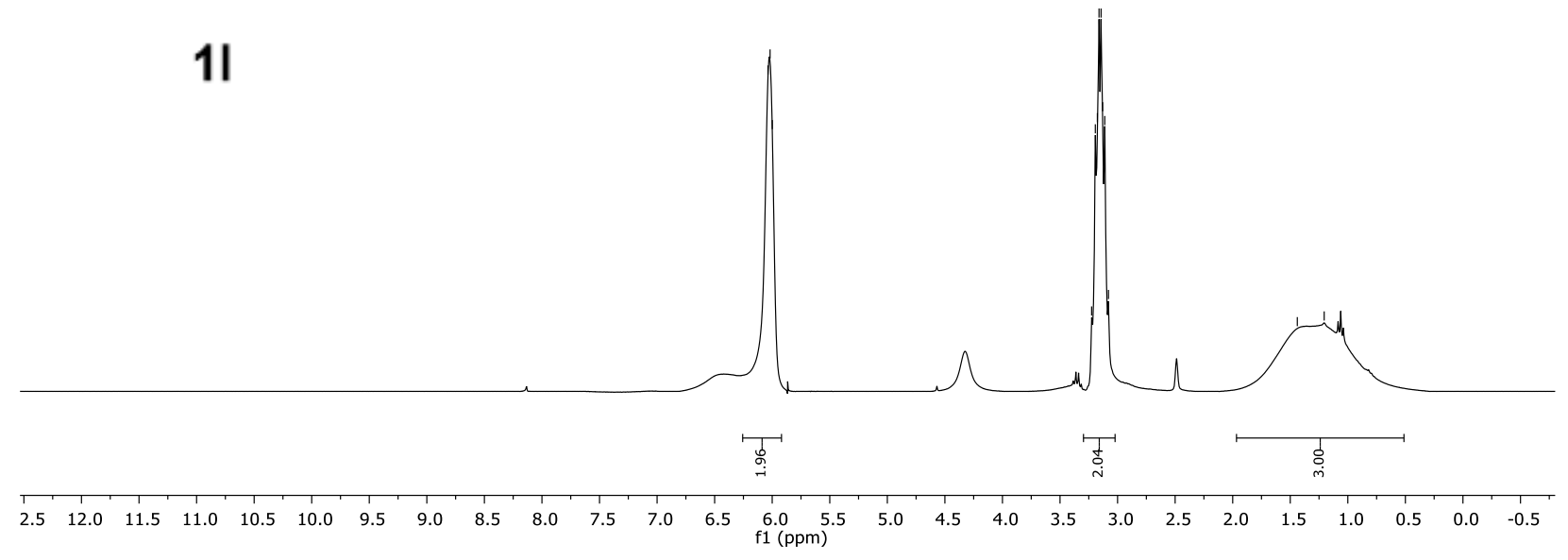

${ }^{1} \mathrm{H}$ NMR (300 MHz, Chloroform-d/ DMSO-d6) 2,2,2-trifluoroethylamine-borane (11)

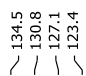

$\mathrm{F}_{\mathrm{F}}^{\mathrm{F}} \mathrm{N}_{2}$

11

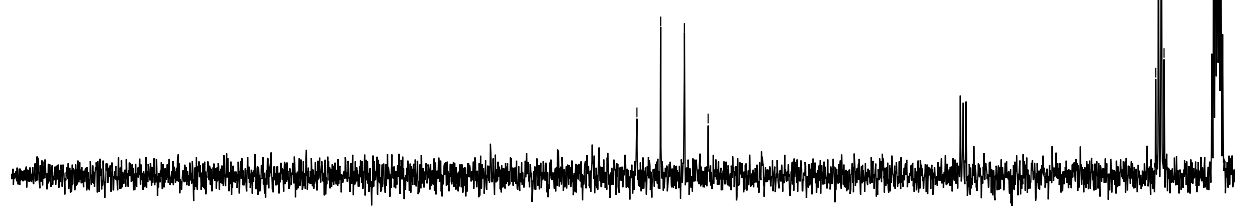

$230 \quad 220 \quad 210$

${ }^{13} \mathrm{C}$ NMR (75 MHz, Chloroform- $d /$ DMSO- $d 6$ ) 2,2,2-trifluoroethylamine-borane (11) 
<smiles>[B]NCC(F)(F)F</smiles>

11
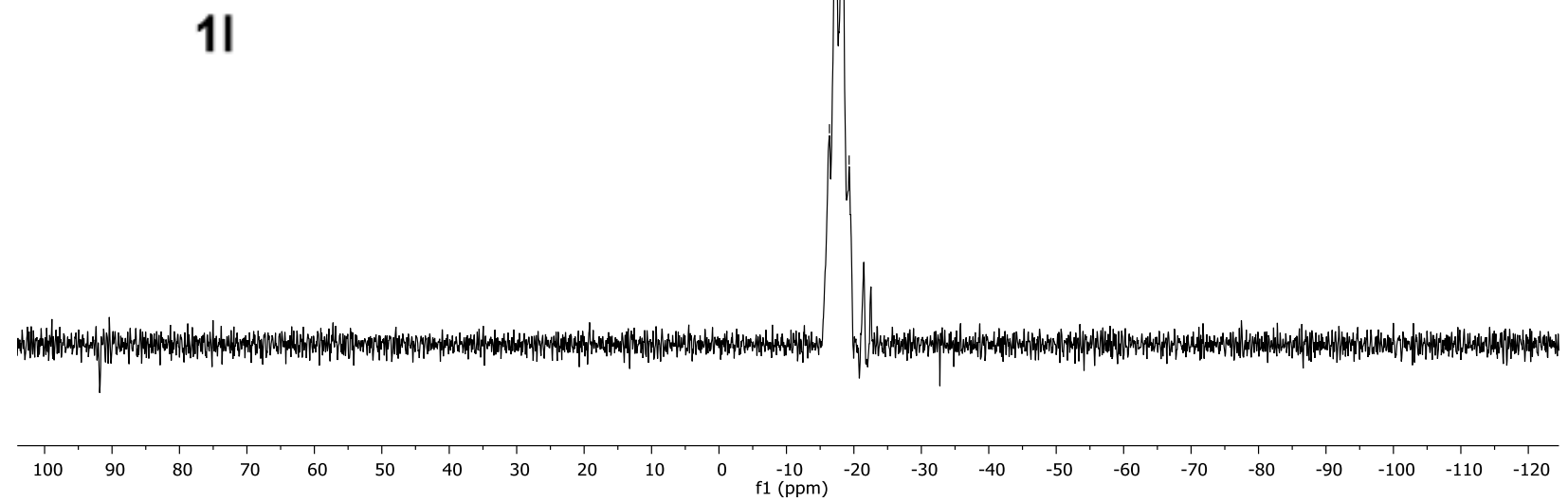

${ }^{11} \mathrm{~B}$ NMR (96 MHz, Chloroform- $d /$ DMSO-d6) 2,2,2-trifluoroethylamine-borane (11)
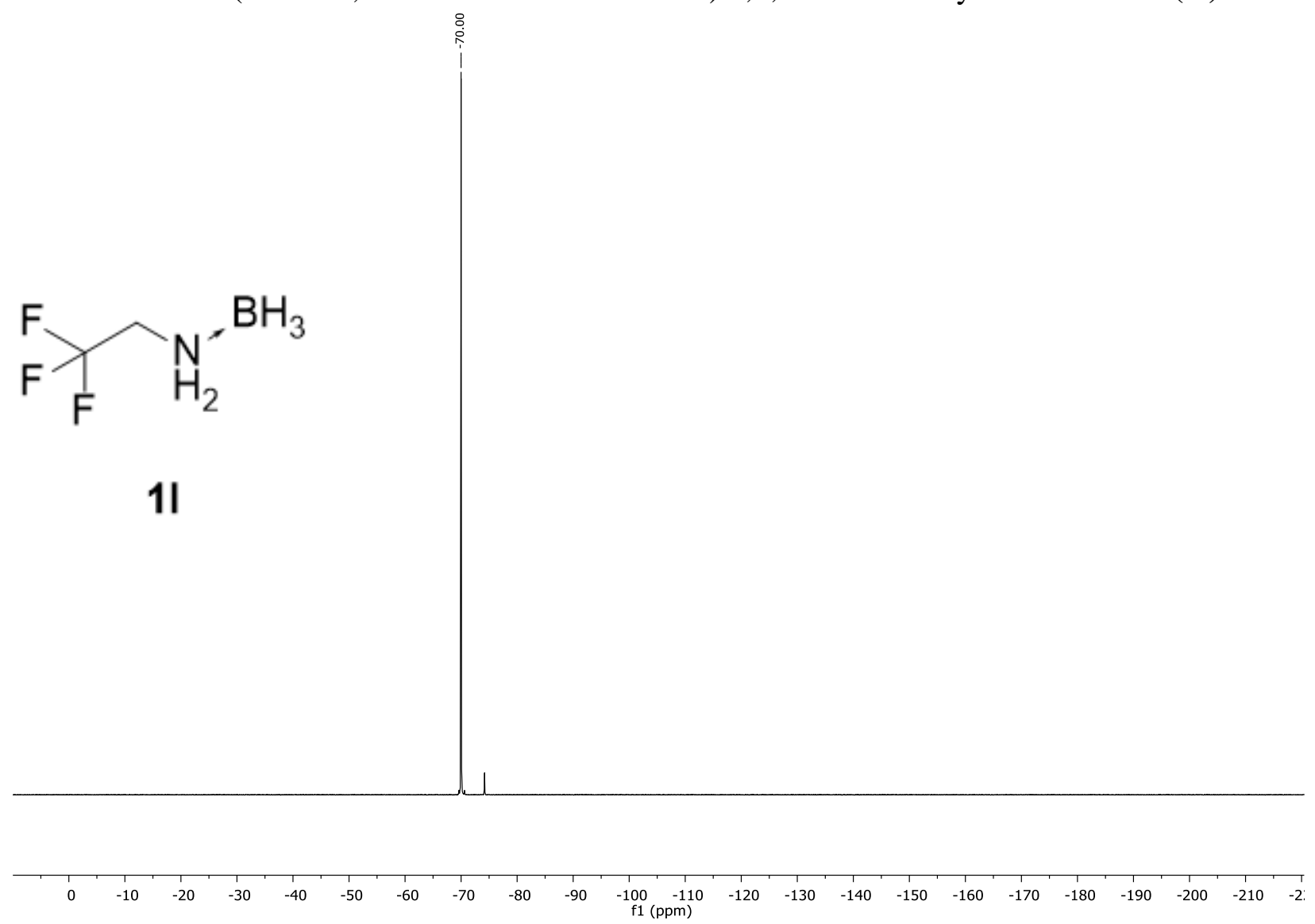

${ }^{19}$ F NMR (282 MHz, Chloroform- $d /$ DMSO- $d 6$ ) 2,2,2-trifluoroethylamine-borane (11) 


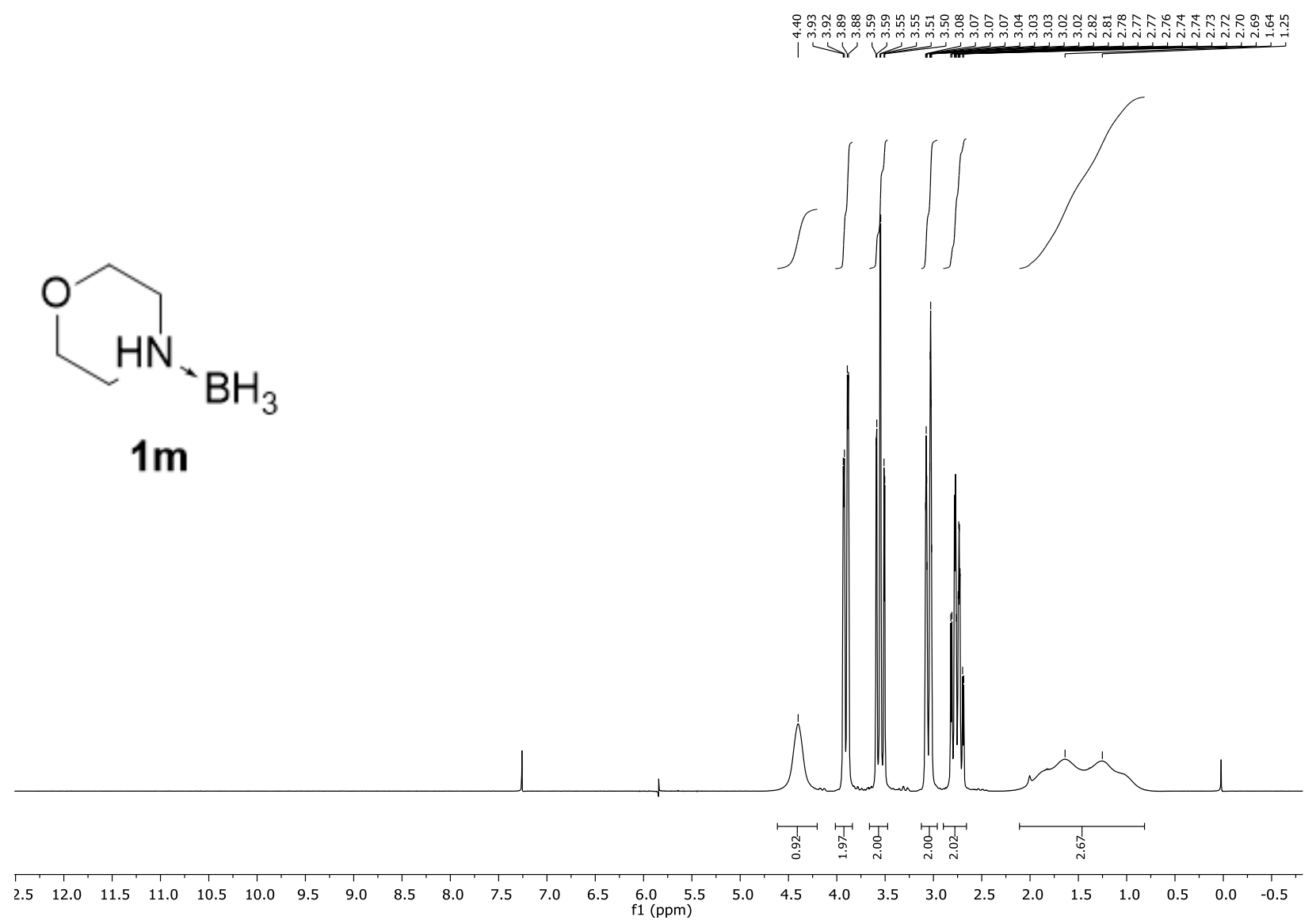

${ }^{1} \mathrm{H}$ NMR (300 MHz, Chloroform- $d$ ) morpholine-borane (1m)

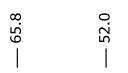

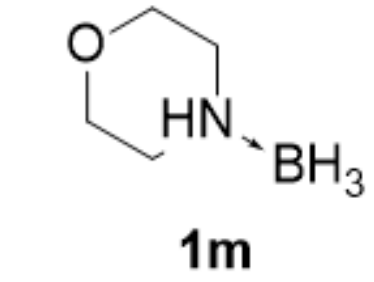

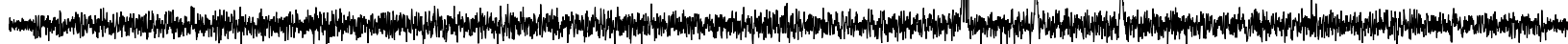

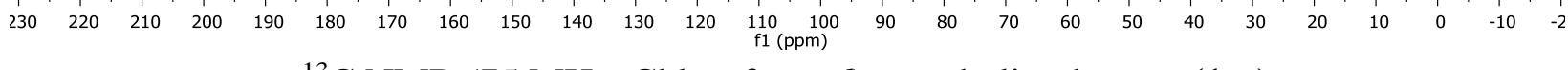

${ }^{13} \mathrm{C}$ NMR (75 MHz, Chloroform- $d$ ) morpholine-borane (1m) 


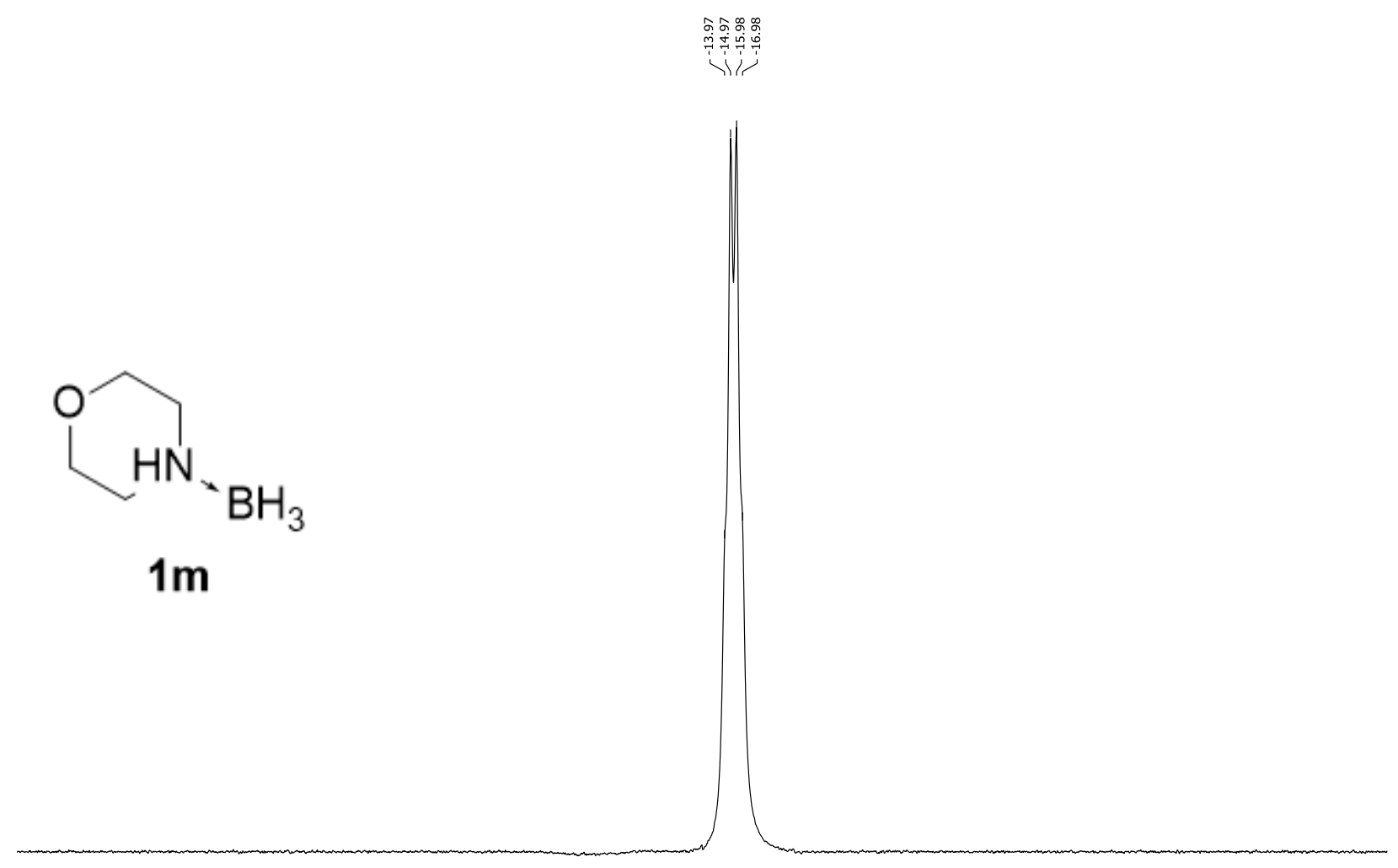

\begin{tabular}{|c|c|c|c|c|c|c|c|c|c|c|c|c|c|c|c|c|c|c|c|c|c|c|}
\hline 100 & 90 & 80 & 70 & 60 & 50 & 40 & 30 & 20 & 10 & 0 & $\begin{array}{c}-10 \\
\mathrm{f} 1(\mathrm{ppm})\end{array}$ & -20 & -30 & -40 & -50 & -60 & -70 & -80 & -90 & -100 & -110 & -120 \\
\hline
\end{tabular}

${ }^{11}$ B NMR (96 MHz, Chloroform- $d$ ) morpholine-borane (1m)
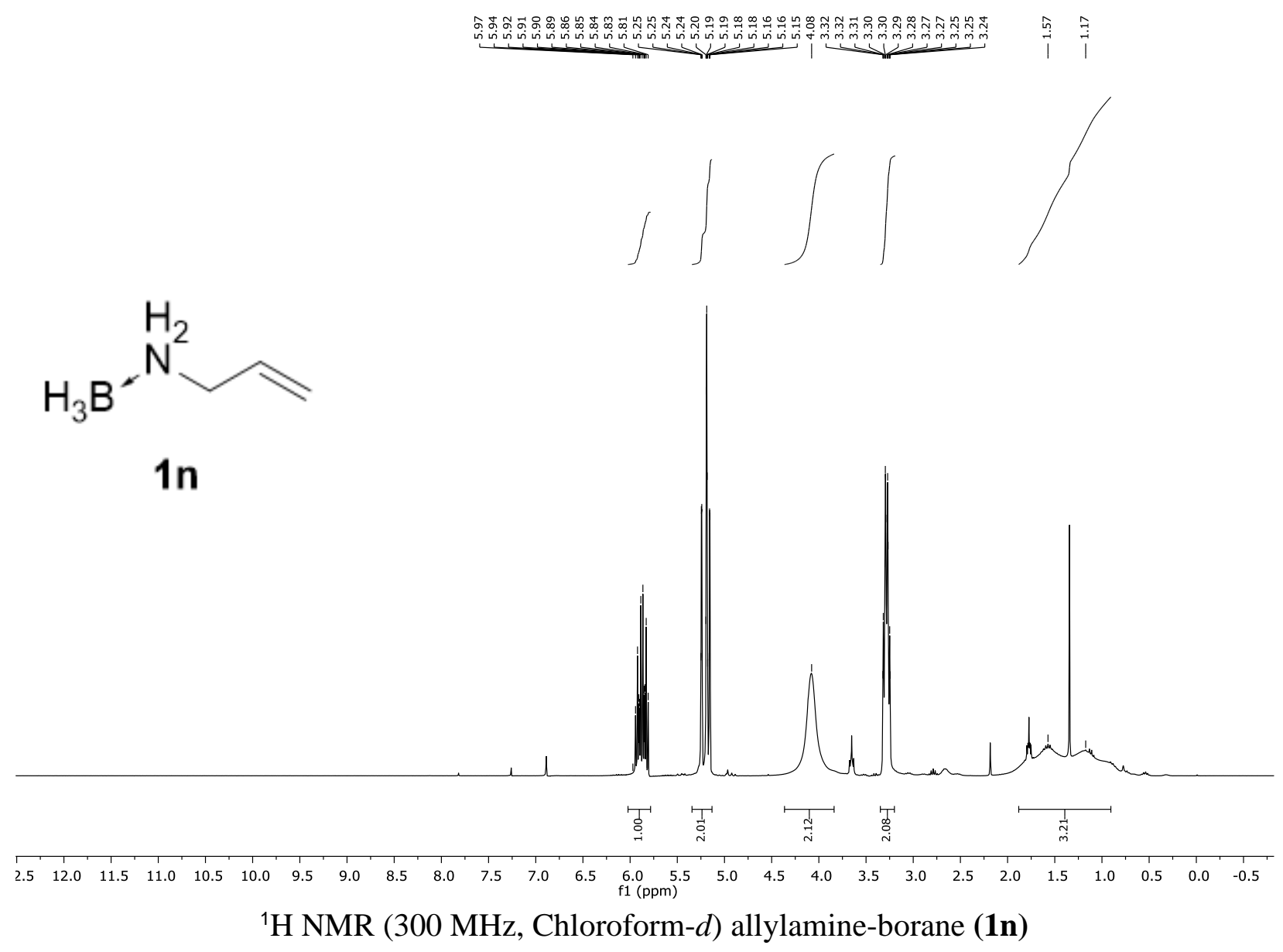


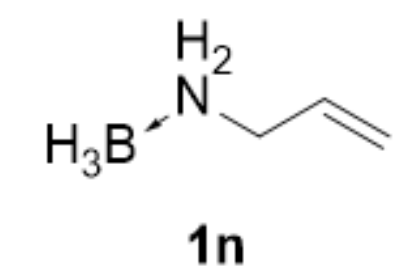

\begin{tabular}{lllllllllllllllllllllllllllll}
\hline 230 & 220 & 210 & 200 & 190 & 180 & 170 & 160 & 150 & 140 & 130 & 120 & $\begin{array}{c}110 \\
f 1(\mathrm{ppm})\end{array}$ & 100 & 90 & 80 & 70 & 60 & 50 & 40 & 30 & 20 & 10 & 0 & -10 & -2
\end{tabular}

${ }^{13} \mathrm{C}$ NMR (75 MHz, Chloroform- $d$ ) allylamine-borane (1n)
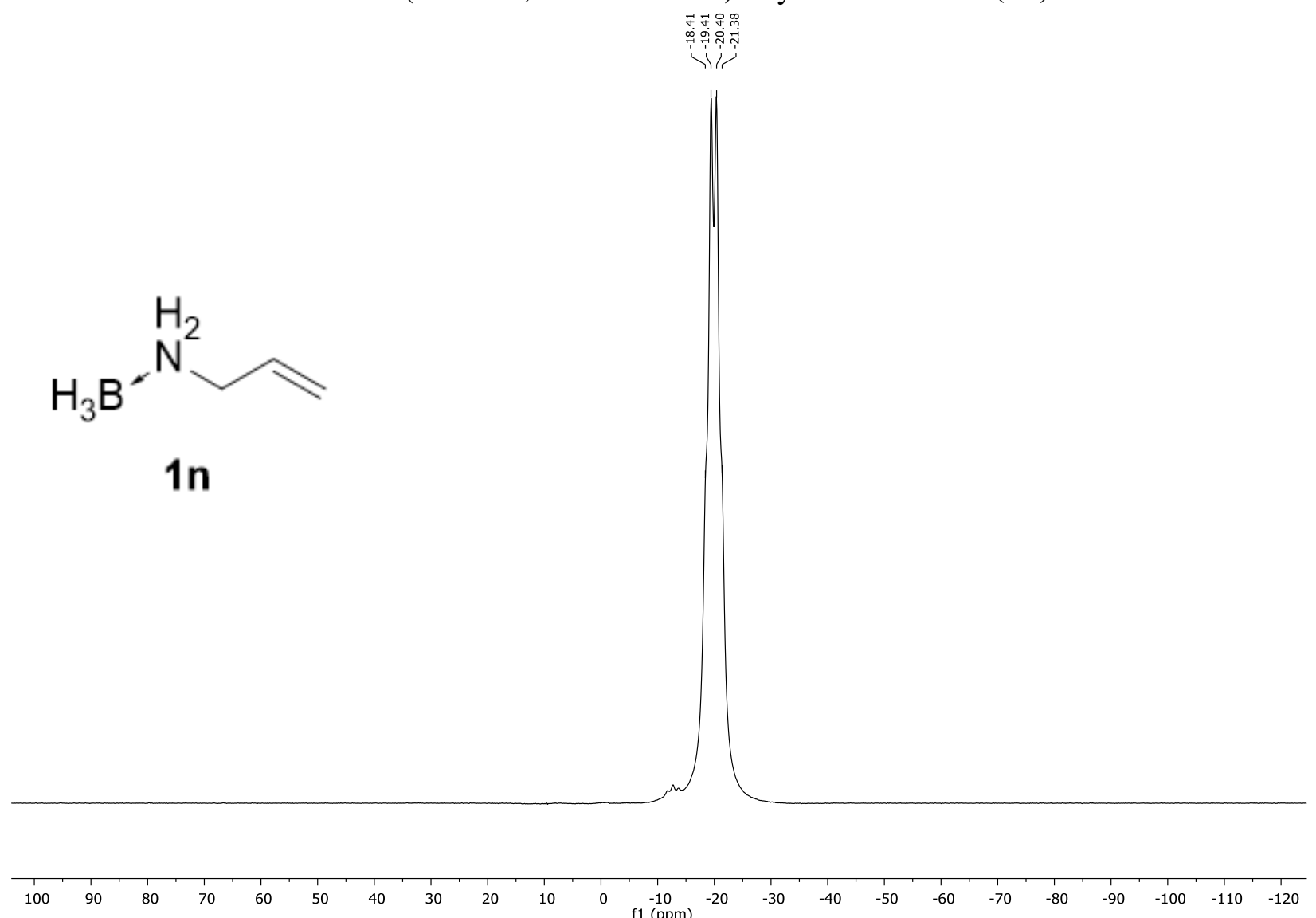

${ }^{11}$ B NMR (96 MHz, Chloroform- $d$ ) allylamine-borane (1n) 


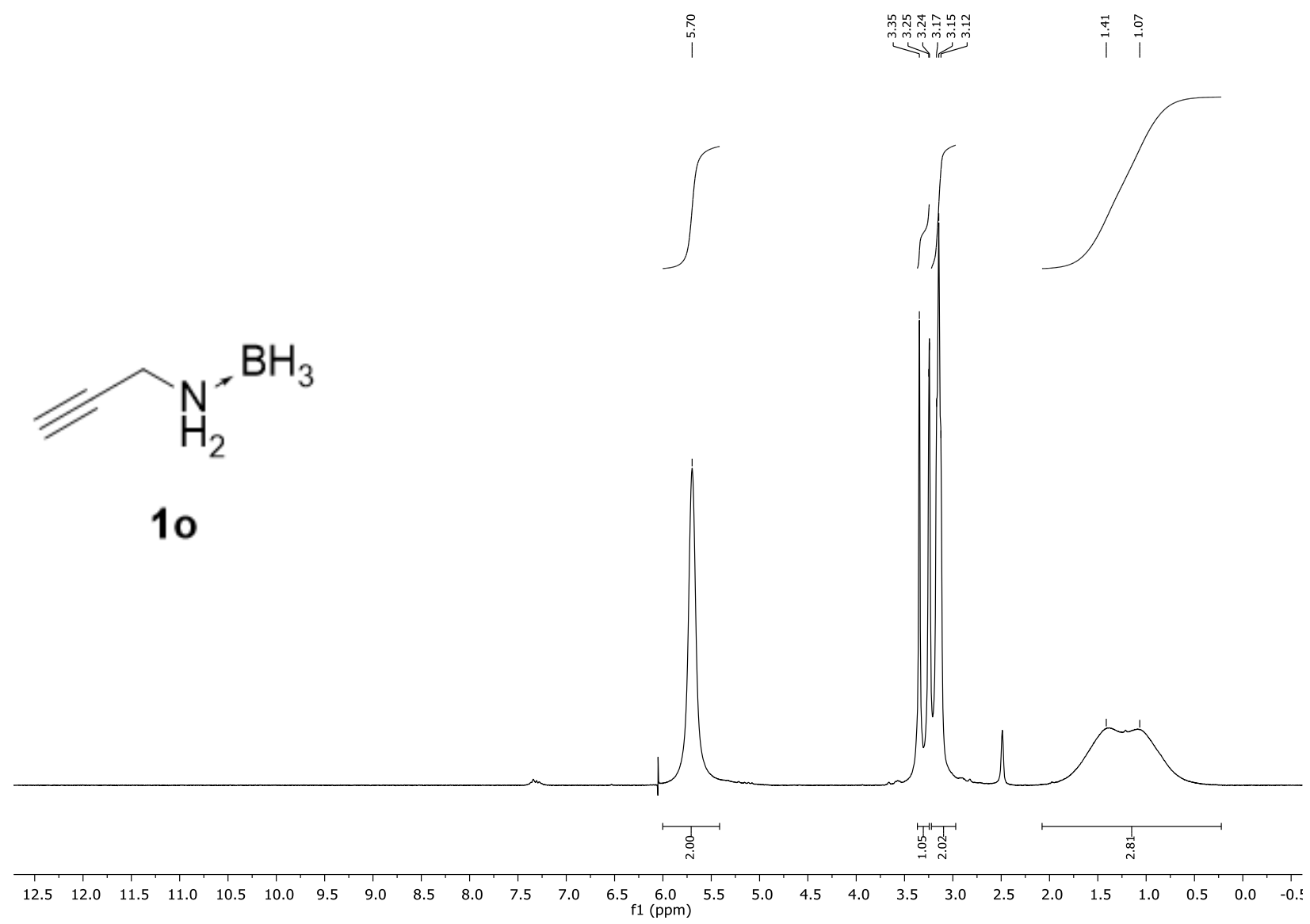

${ }^{1} \mathrm{H}$ NMR (300 MHz, DMSO-d6) propargylamine-borane (1o)

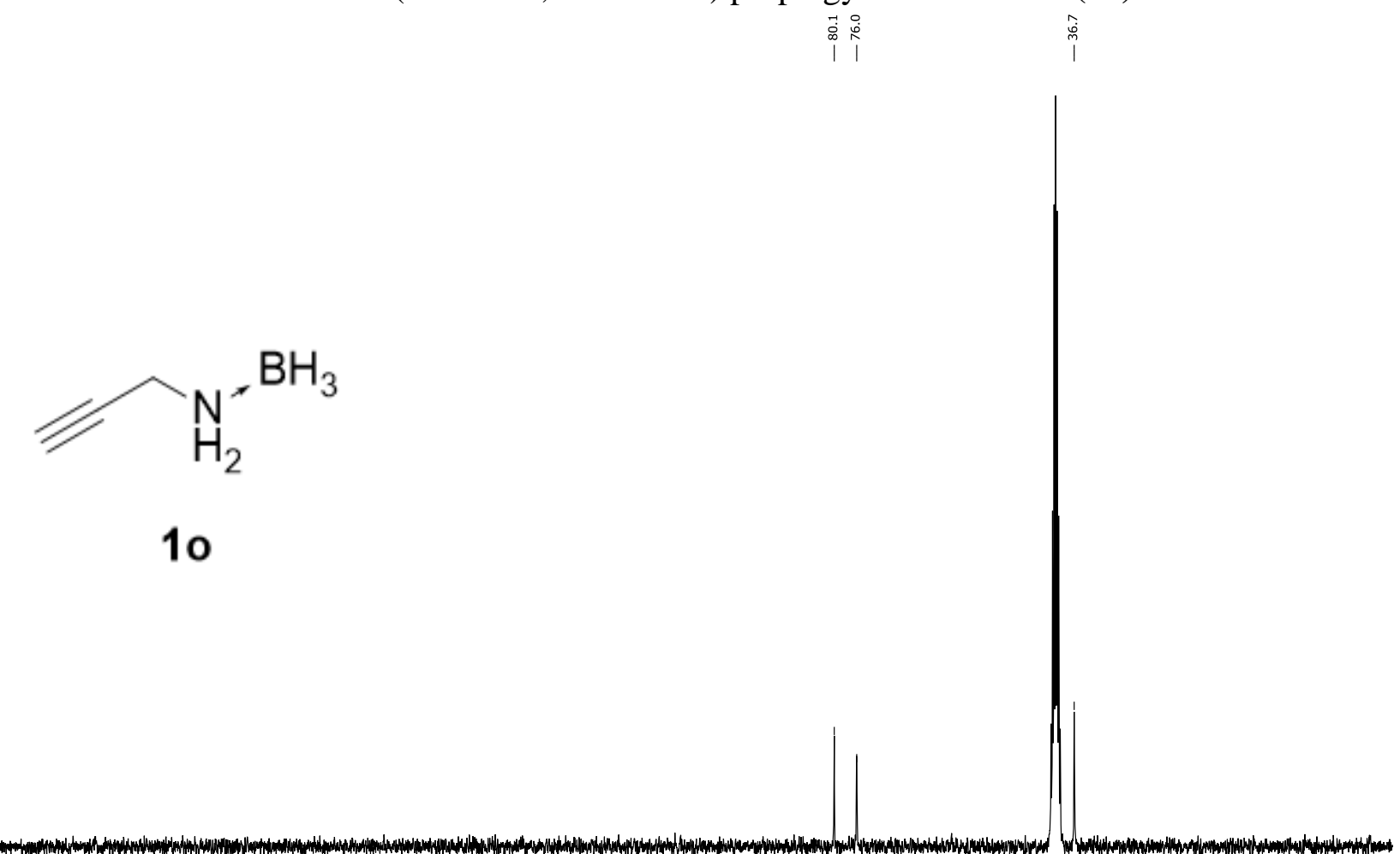

$\begin{array}{llllllllllllllllllllllllll}130 & 220 & 210 & 200 & 190 & 180 & 170 & 160 & 150 & 140 & 130 & 120 & \begin{array}{c}110 \\ f 1\end{array}(\mathrm{ppm}) & 100 & 80 & 70 & 60 & 50 & 40 & 30 & 20 & 10 & 0 & -10 & -2\end{array}$

${ }^{13} \mathrm{C}$ NMR (75 MHz, DMSO- $d 6$ ) propargylamine-borane (1o) 


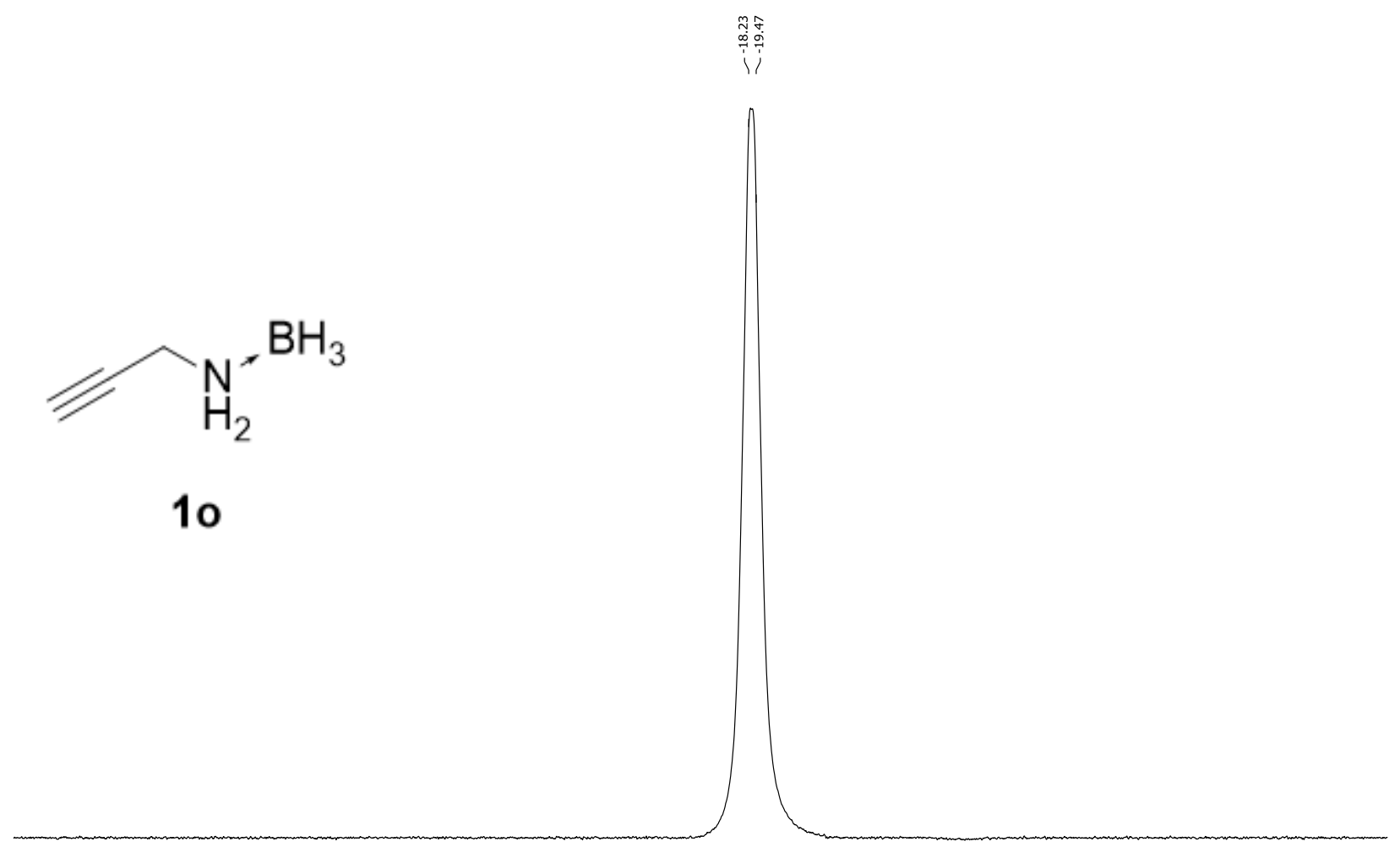

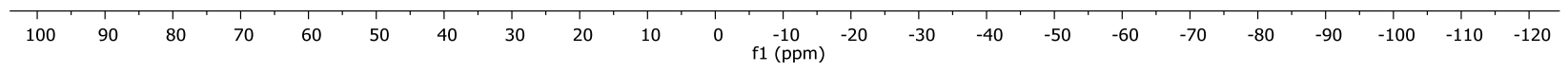

${ }^{11} \mathrm{~B}$ NMR (96 MHz, DMSO-d6) propargylamine-borane (1o)

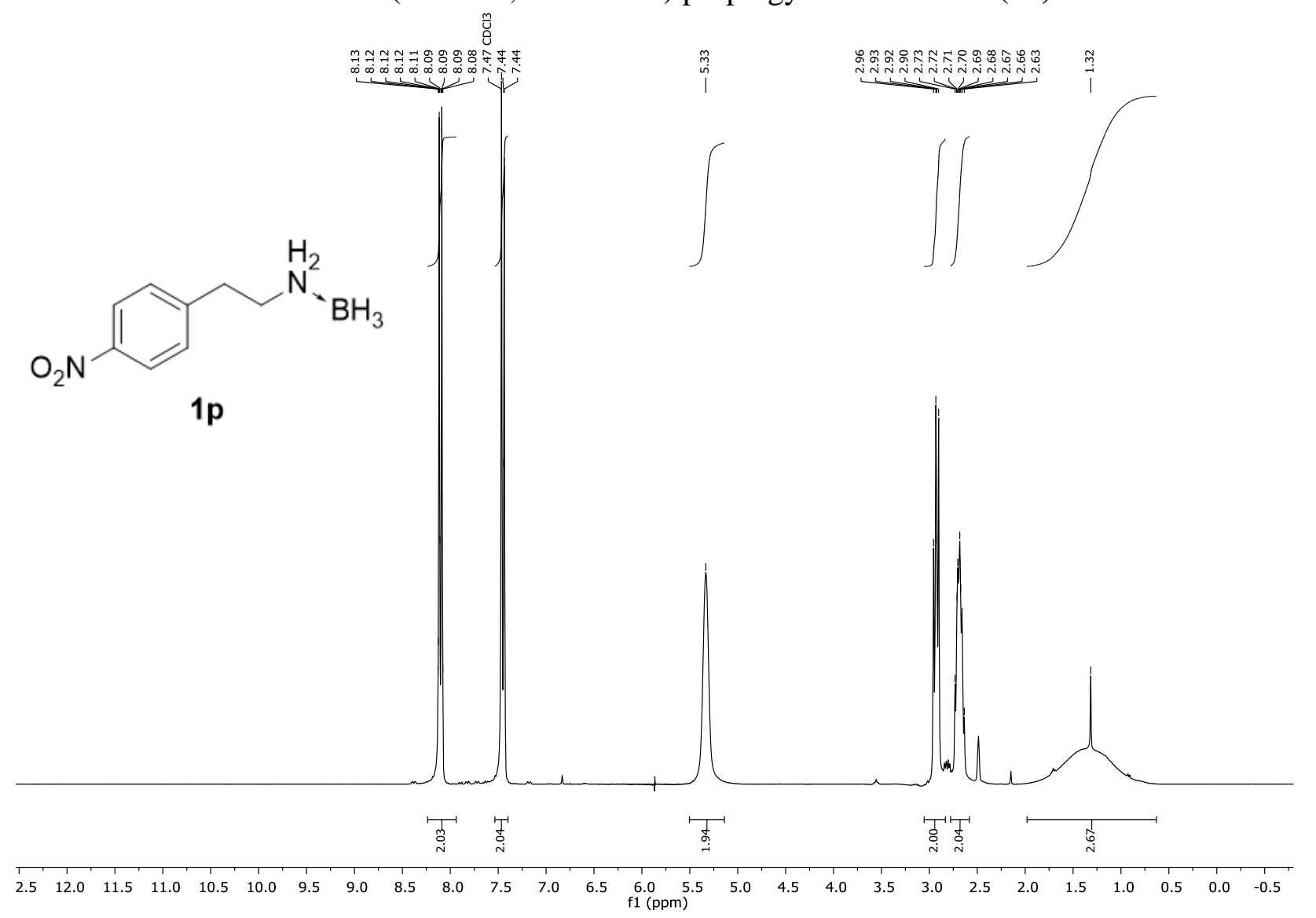

${ }^{1} \mathrm{H}$ NMR (300 MHz, DMSO-d6) 2-(4-nitrophenyl)ethan-1-amine-borane (1p) 


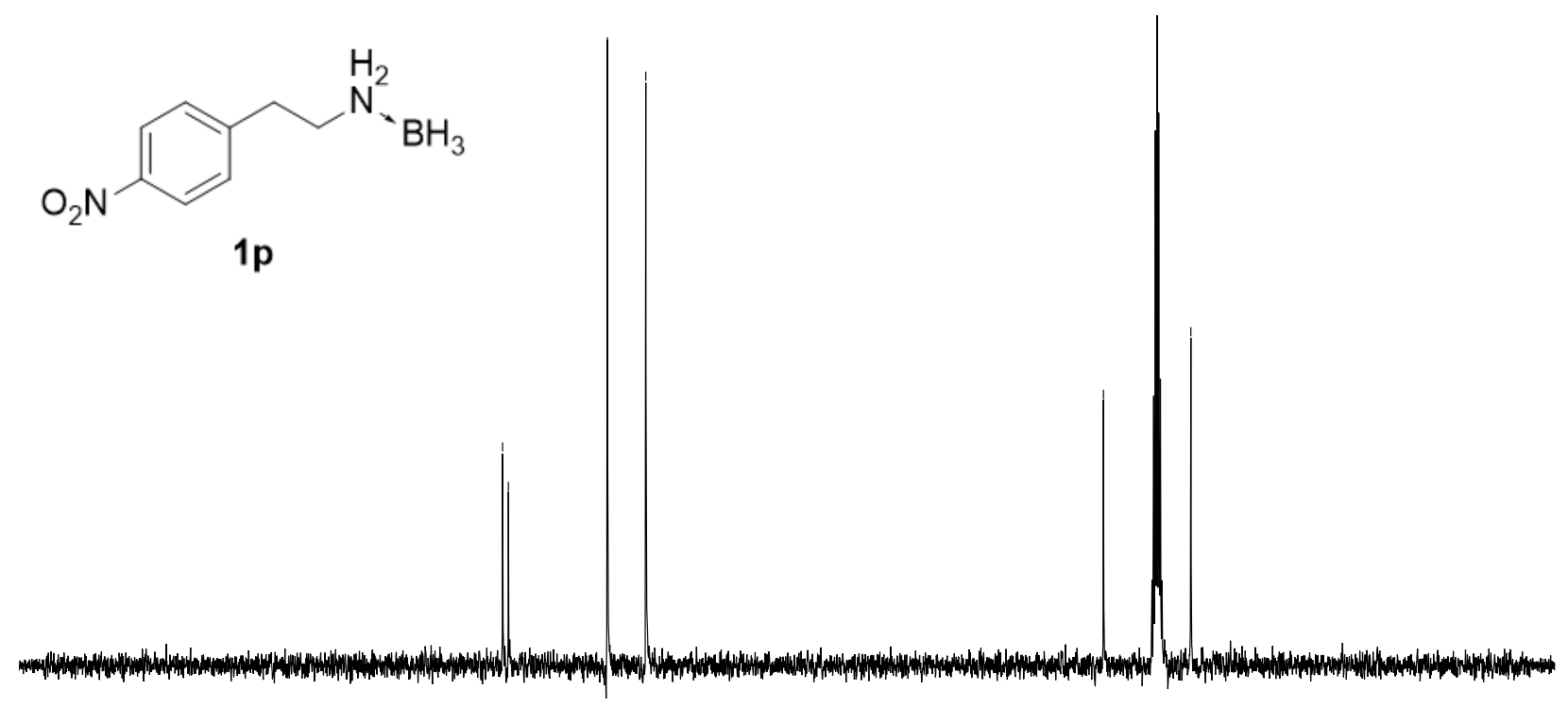

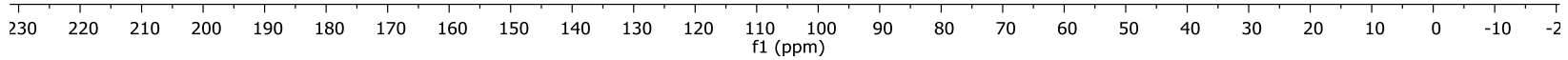

${ }^{13} \mathrm{C}$ NMR (75 MHz, DMSO-d6) 2-(4-nitrophenyl)ethan-1-amine-borane (1p) \%ํํำ<smiles>[B]NCCc1ccc([N+](=O)[O-])cc1</smiles> 


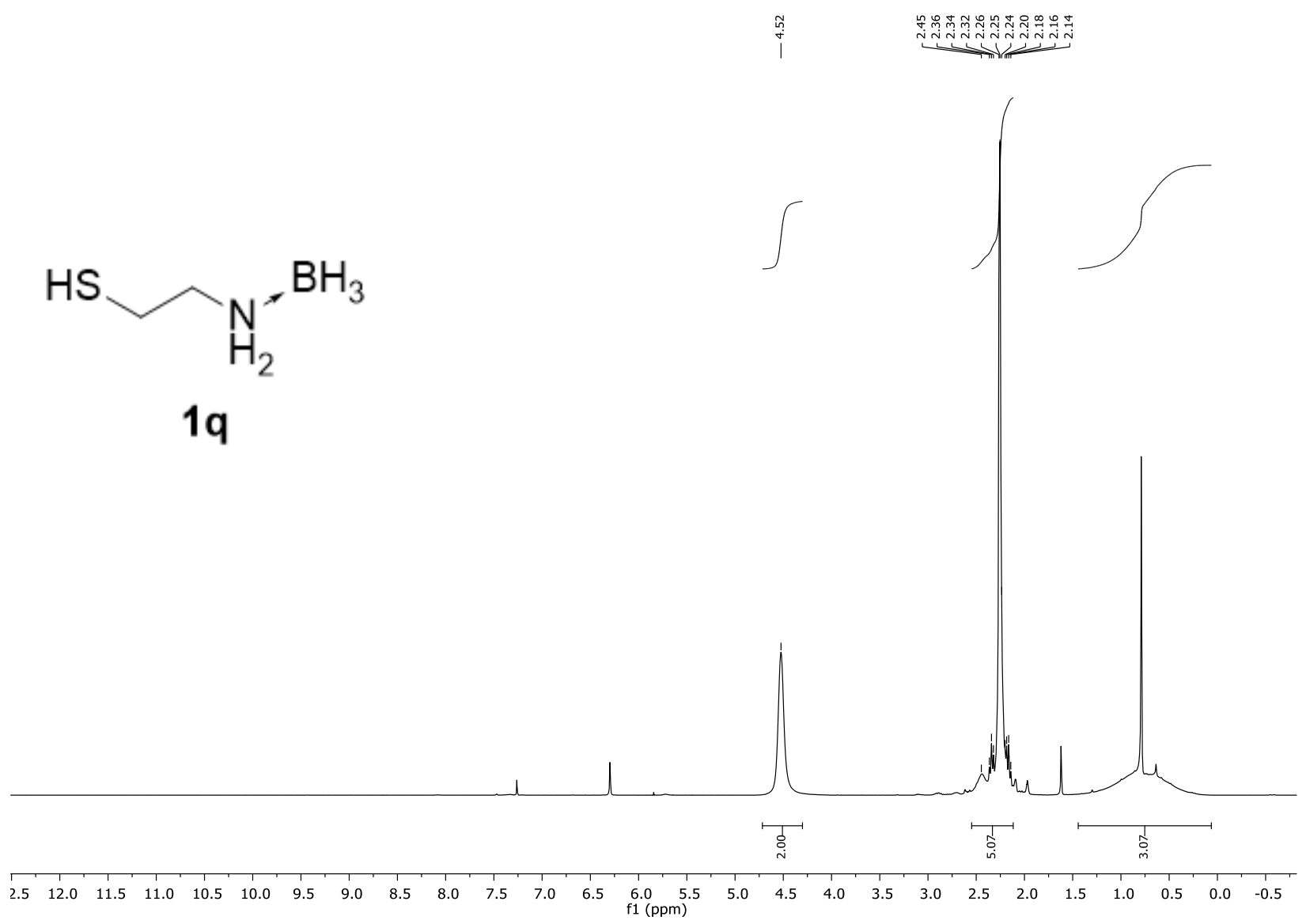

${ }^{1} \mathrm{H}$ NMR (300 MHz, DMSO-d6) 2-aminoethane-1-thiol-borane (1q)

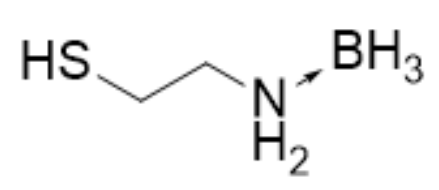

$1 q$
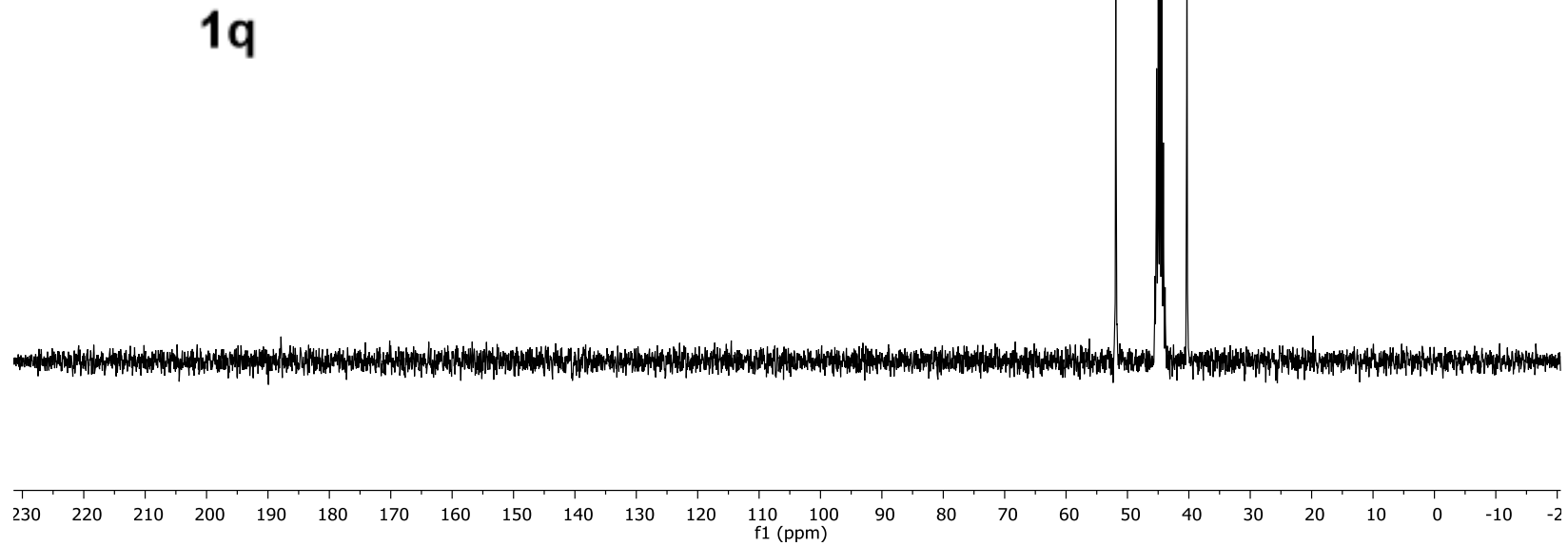

${ }^{13} \mathrm{C}$ NMR (75 MHz, DMSO-d6) 2-aminoethane-1-thiol-borane (1q) 

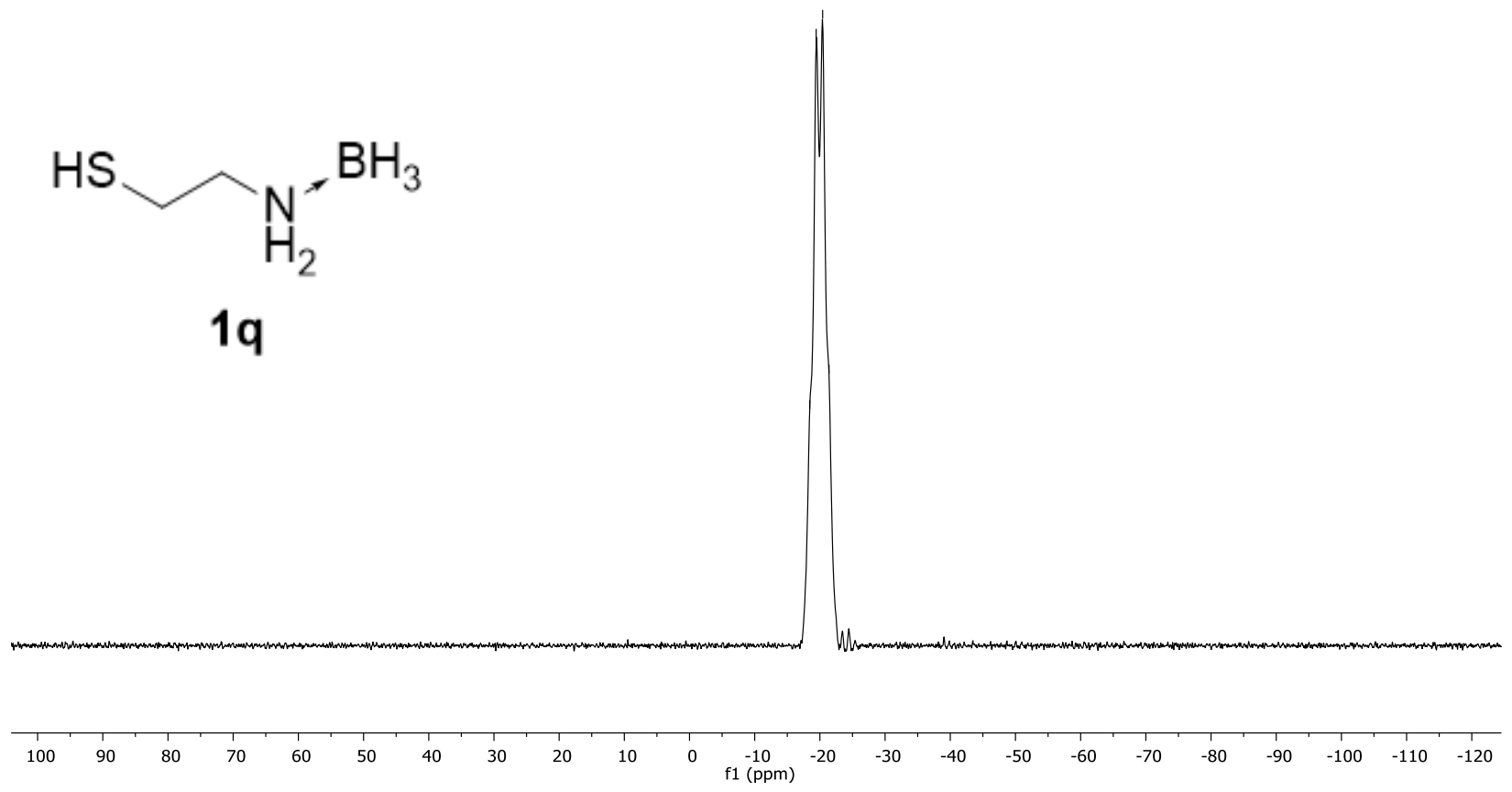

${ }^{11}$ B NMR (96 MHz, DMSO-d6) 2-aminoethane-1-thiol-borane (1q)

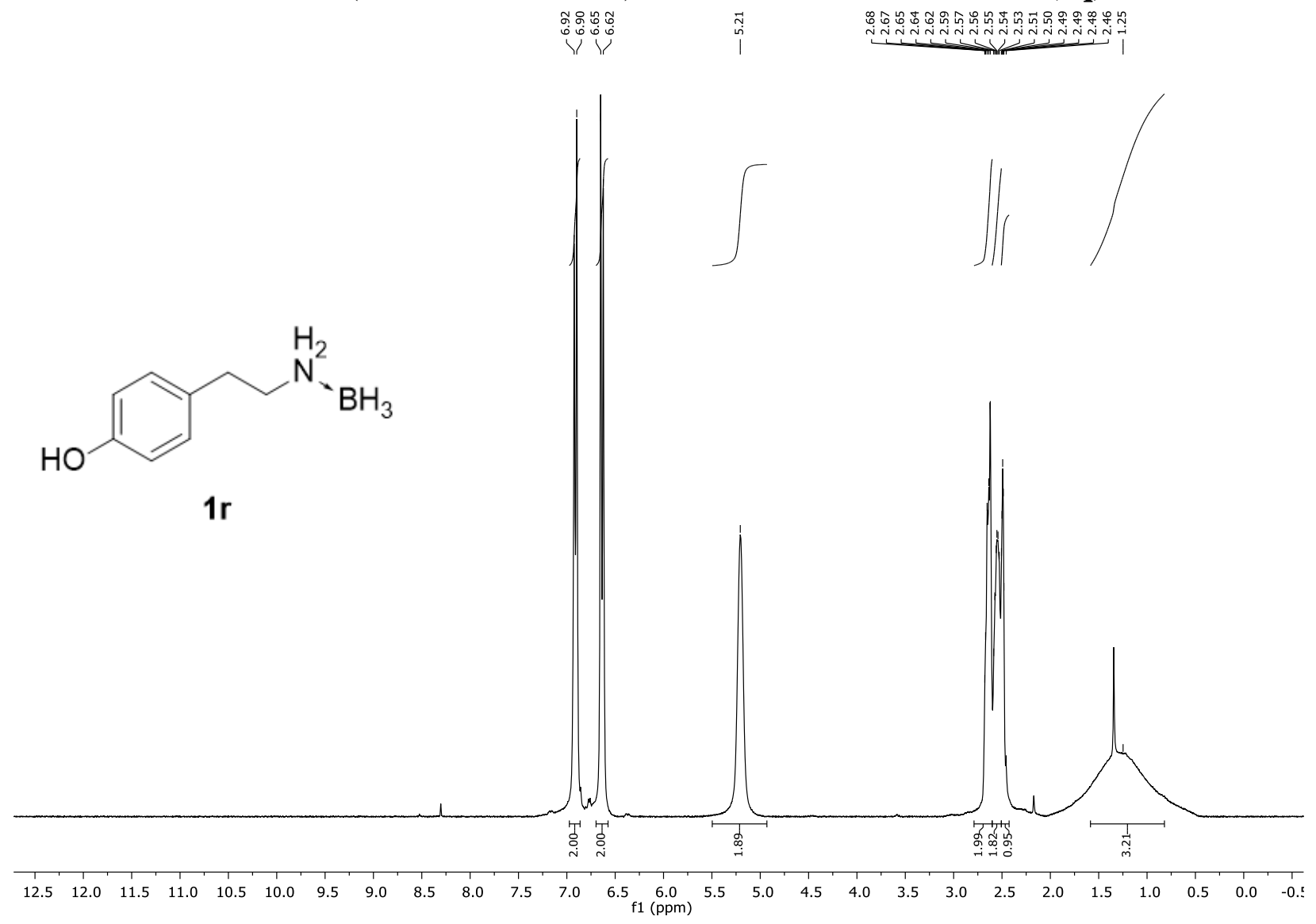

${ }^{1} \mathrm{H}$ NMR (300 MHz, DMSO-d6) tyramine-borane (1r) 

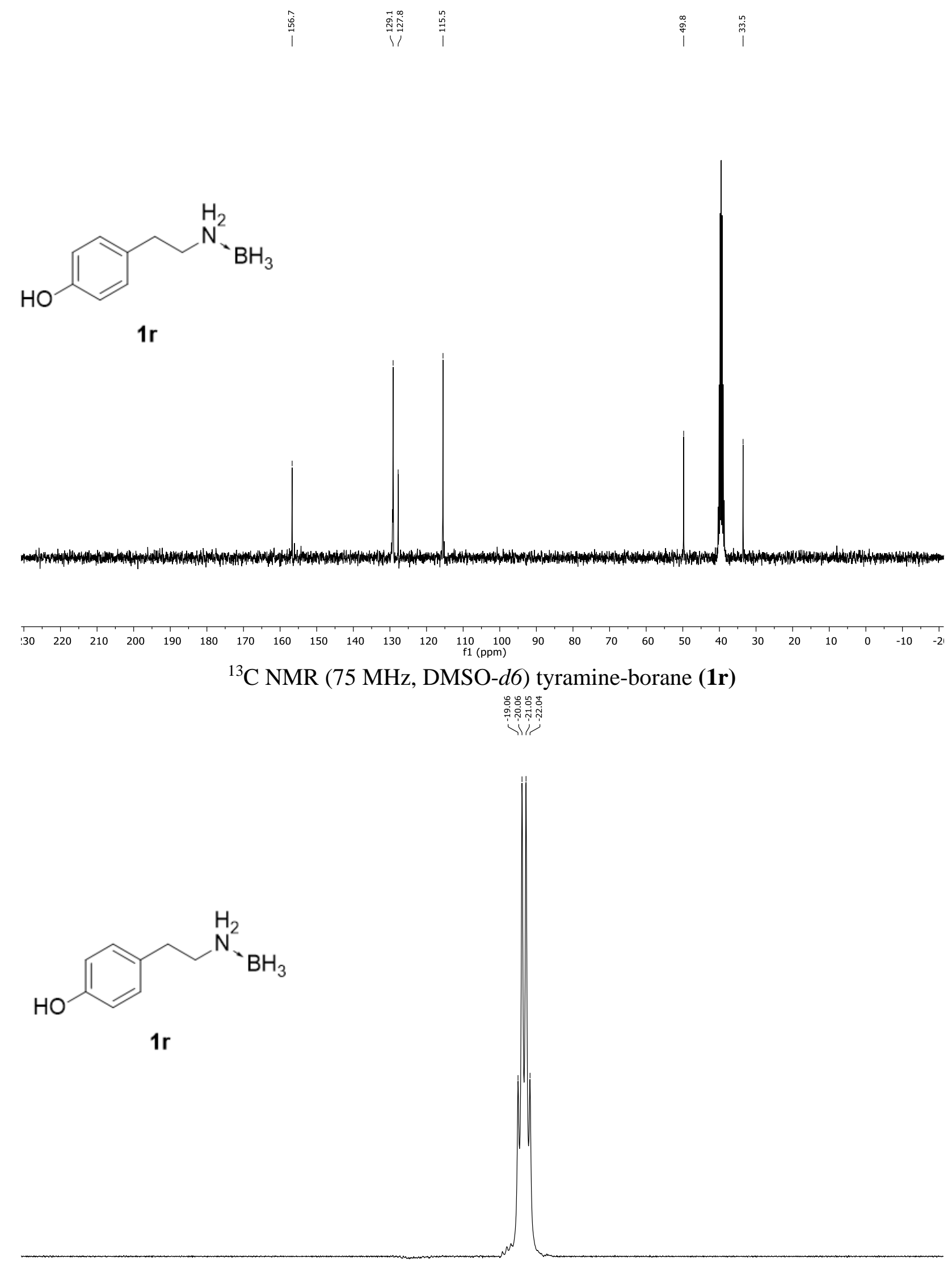

\begin{tabular}{|c|c|c|c|c|c|c|c|c|c|c|c|c|c|c|c|c|c|c|c|c|c|c|}
\hline 100 & 90 & 80 & 70 & 60 & 50 & 40 & 30 & 20 & 10 & 0 & $\begin{array}{c}-10 \\
\mathrm{f} 1(\mathrm{ppm})\end{array}$ & -20 & -30 & -40 & -50 & -60 & -70 & -80 & -90 & -100 & -110 & -120 \\
\hline
\end{tabular}




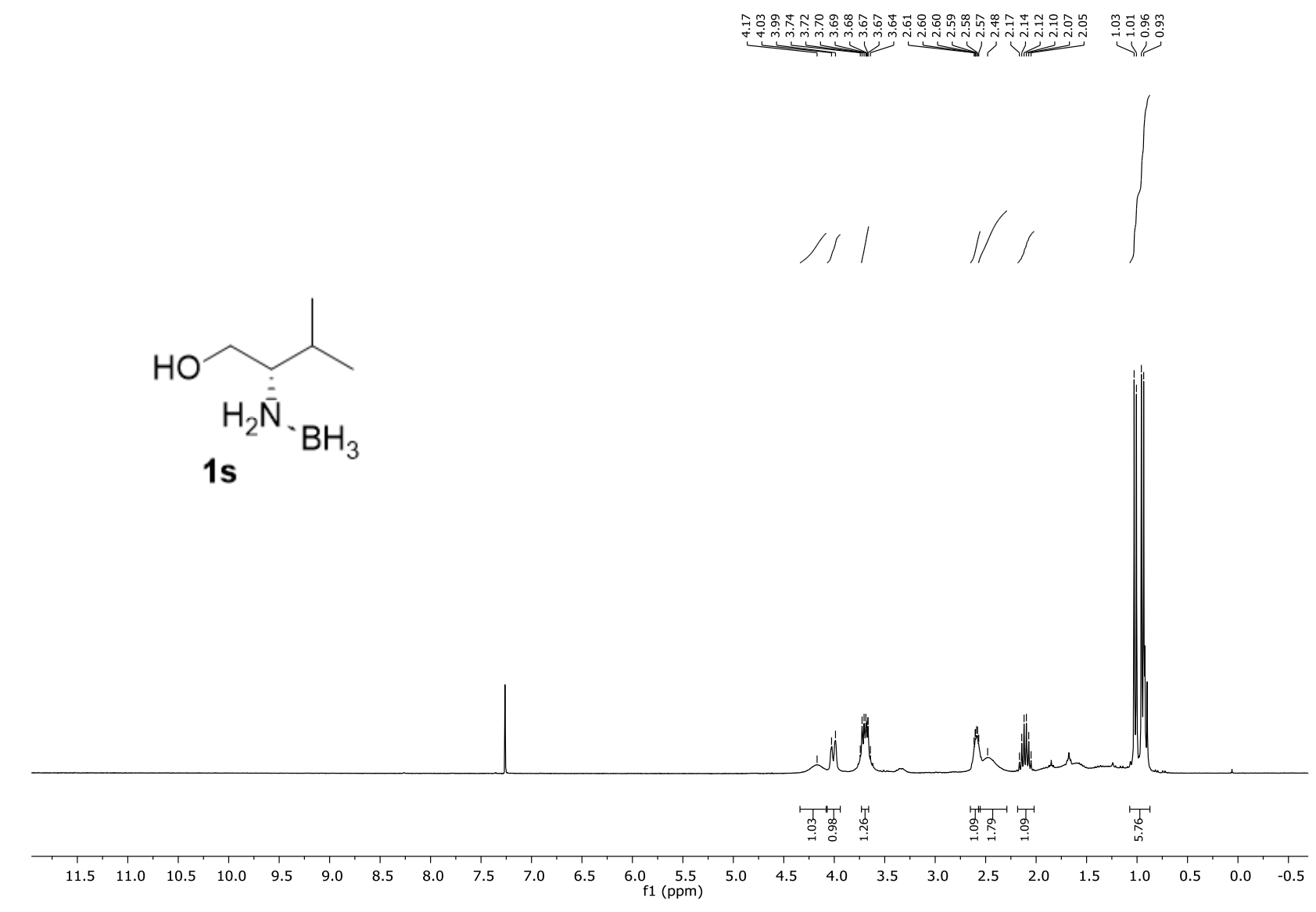

${ }^{1} \mathrm{H}$ NMR (300 MHz, Chloroform-d) valinol-borane (1s)

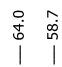

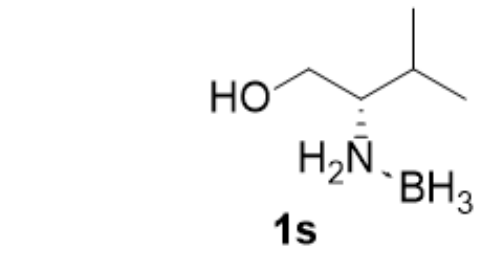

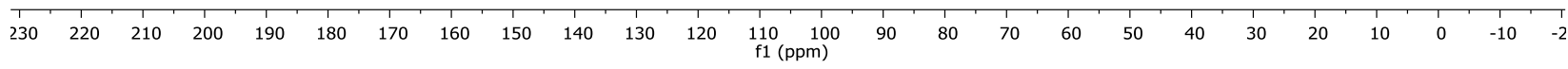

${ }^{13} \mathrm{C}$ NMR (75 MHz, Chloroform- $d$ ) valinol-borane (1s) 

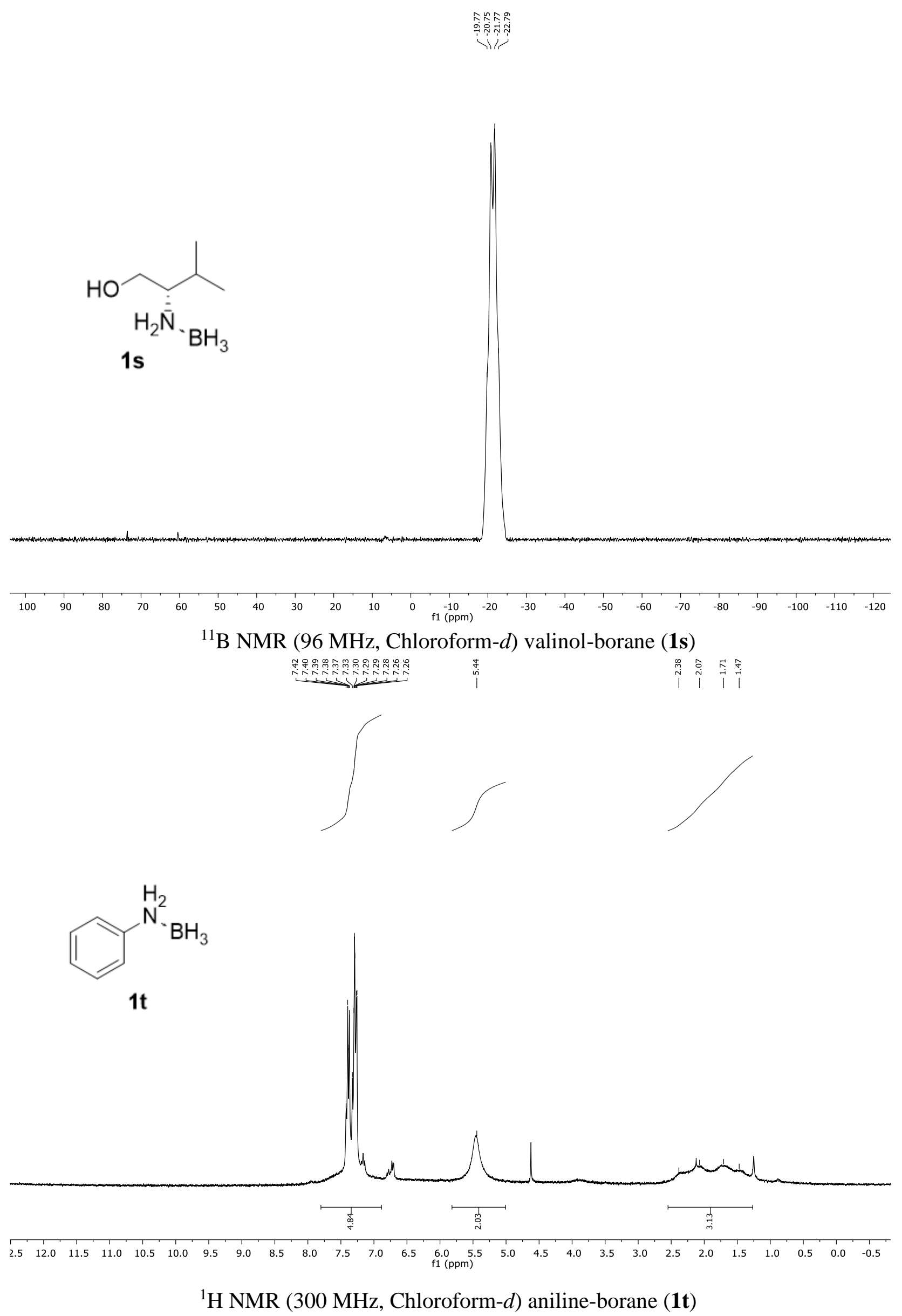

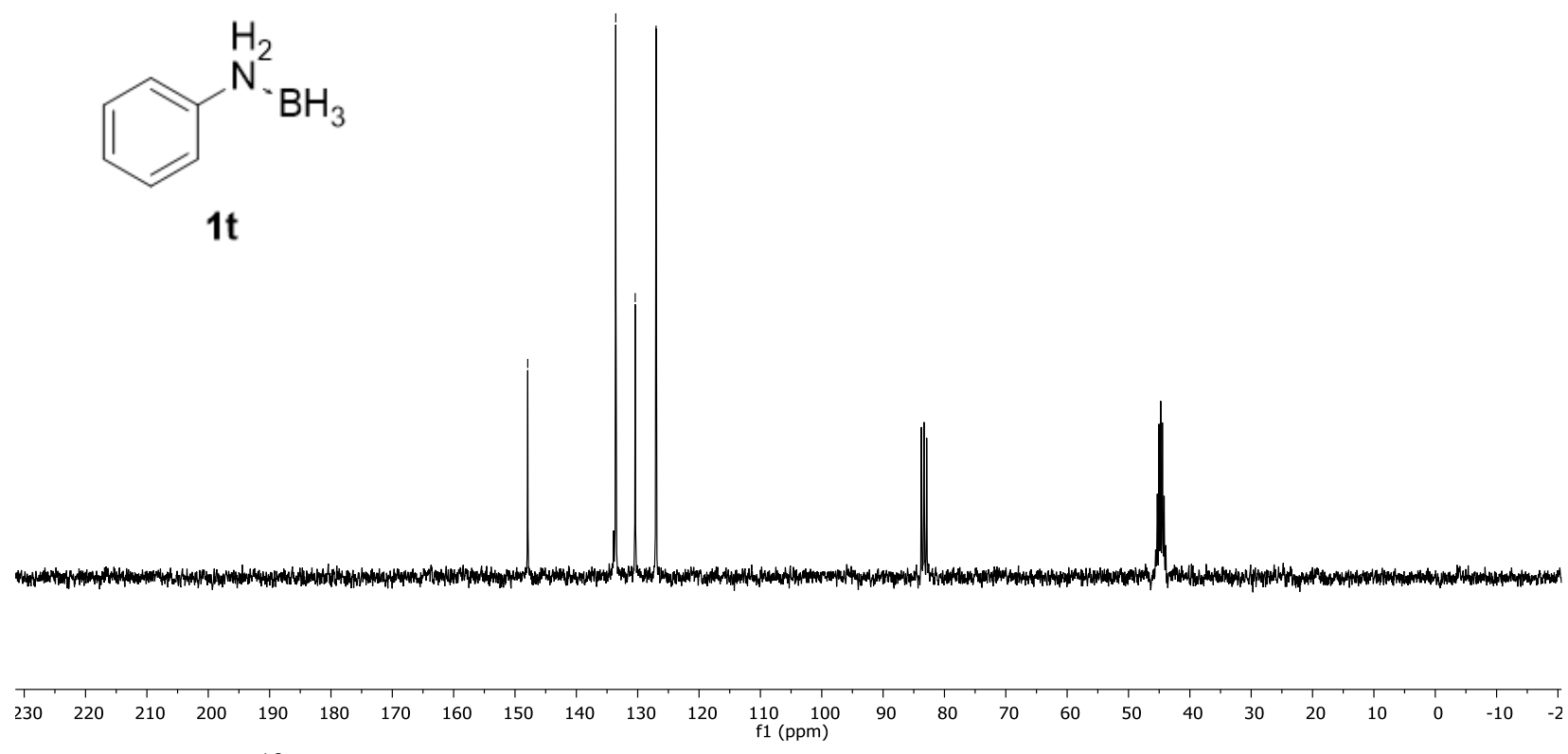

${ }^{13} \mathrm{C}$ NMR (75 MHz, Chloroform- $d /$ DMSO- $d 6$ ) aniline-borane (1t)

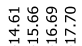
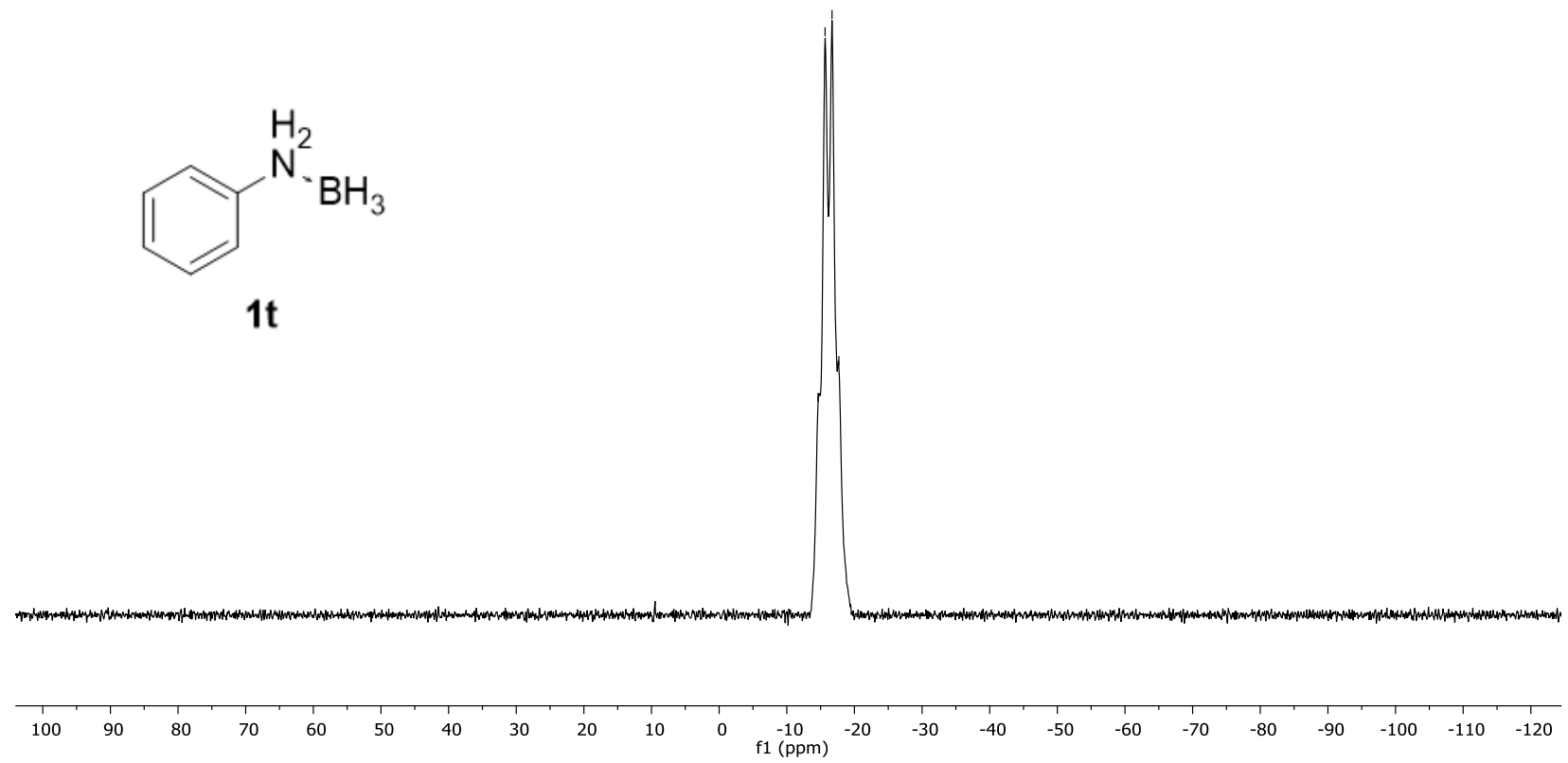

${ }^{11}$ B NMR (96 MHz, Chloroform- $d$ ) aniline-borane (1t) 
NMR spectra of amide products:

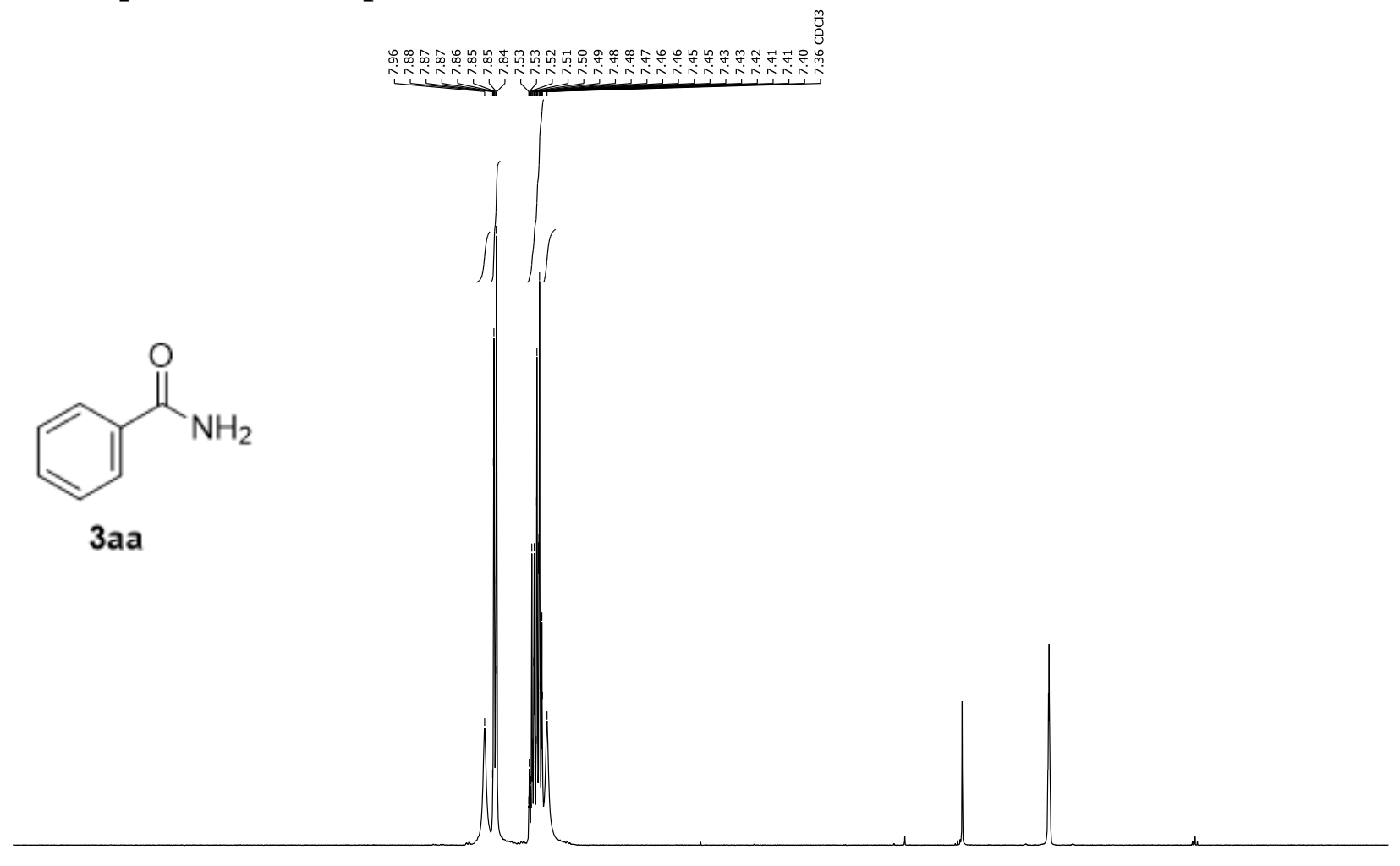

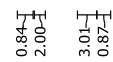

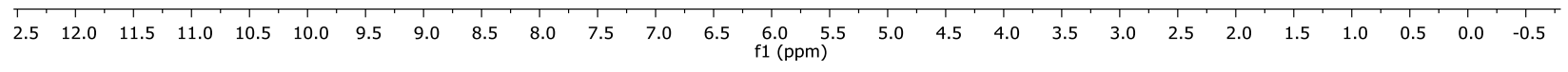

${ }^{1} \mathrm{H}$ NMR (300 MHz, DMSO-d6) benzamide (3aa)

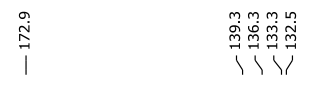<smiles>NC(=O)c1ccccc1</smiles>

3aa

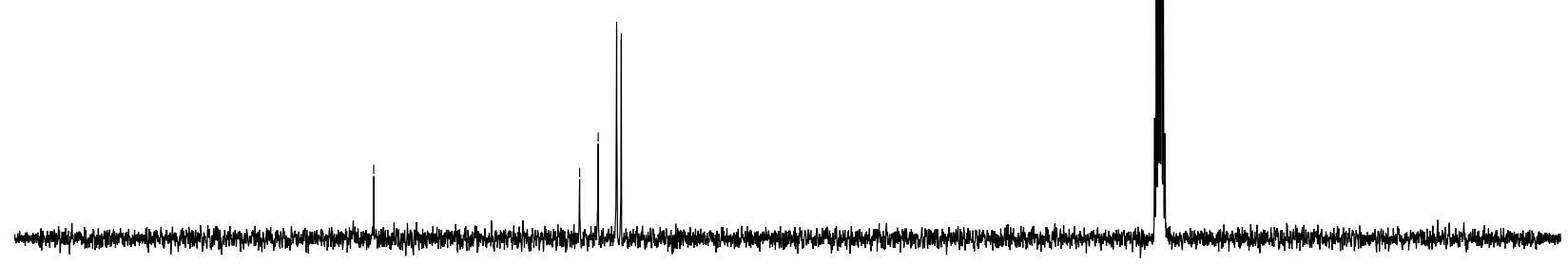

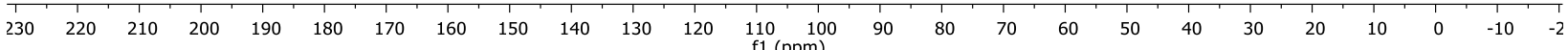

${ }^{13} \mathrm{C}$ NMR (75 MHz, DMSO- $d 6$ ) benzamide (3aa) 


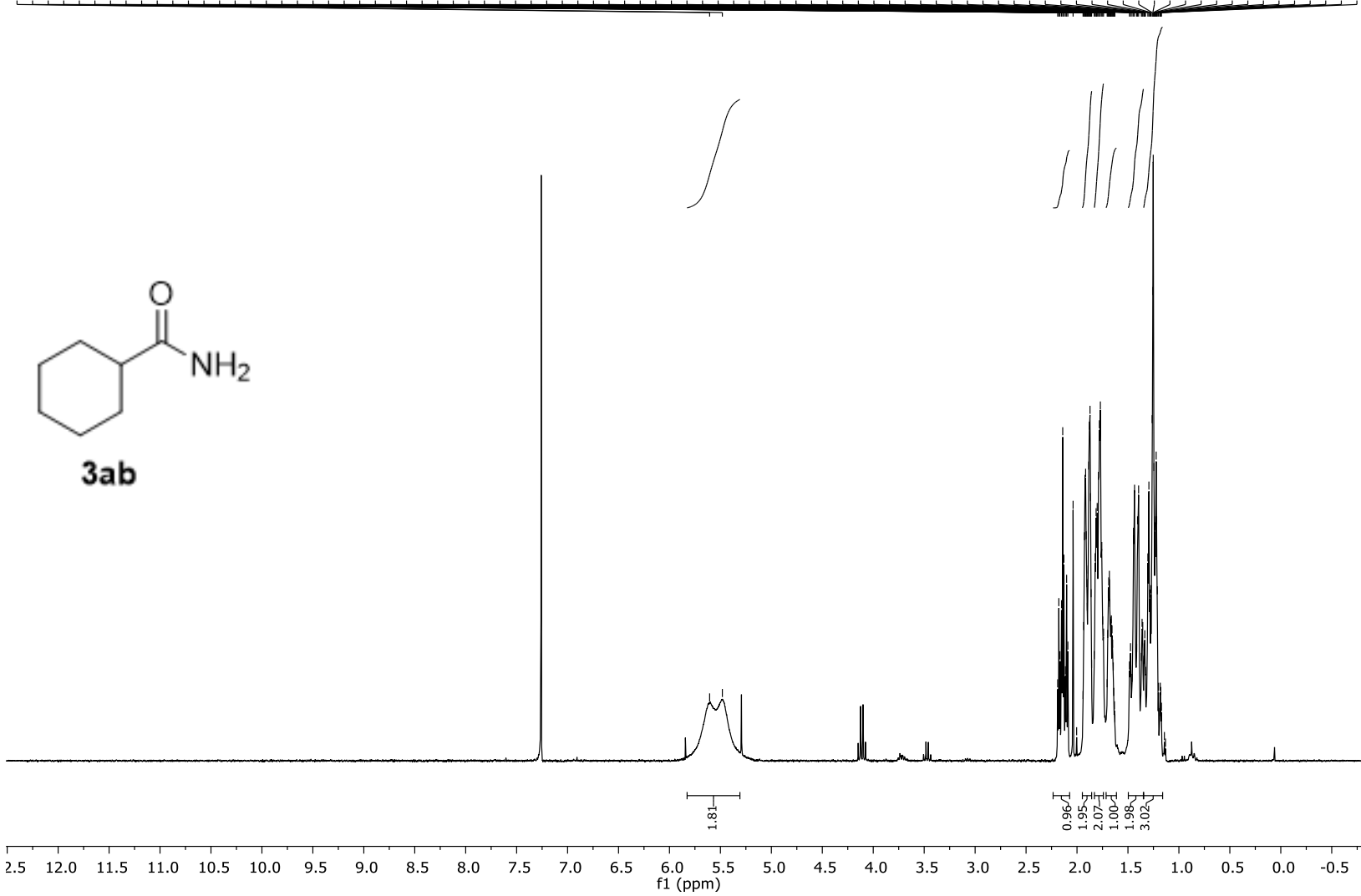

${ }^{1} \mathrm{H}$ NMR (300 MHz, DMSO-d6) cyclohexanecarboxamide (3ab)

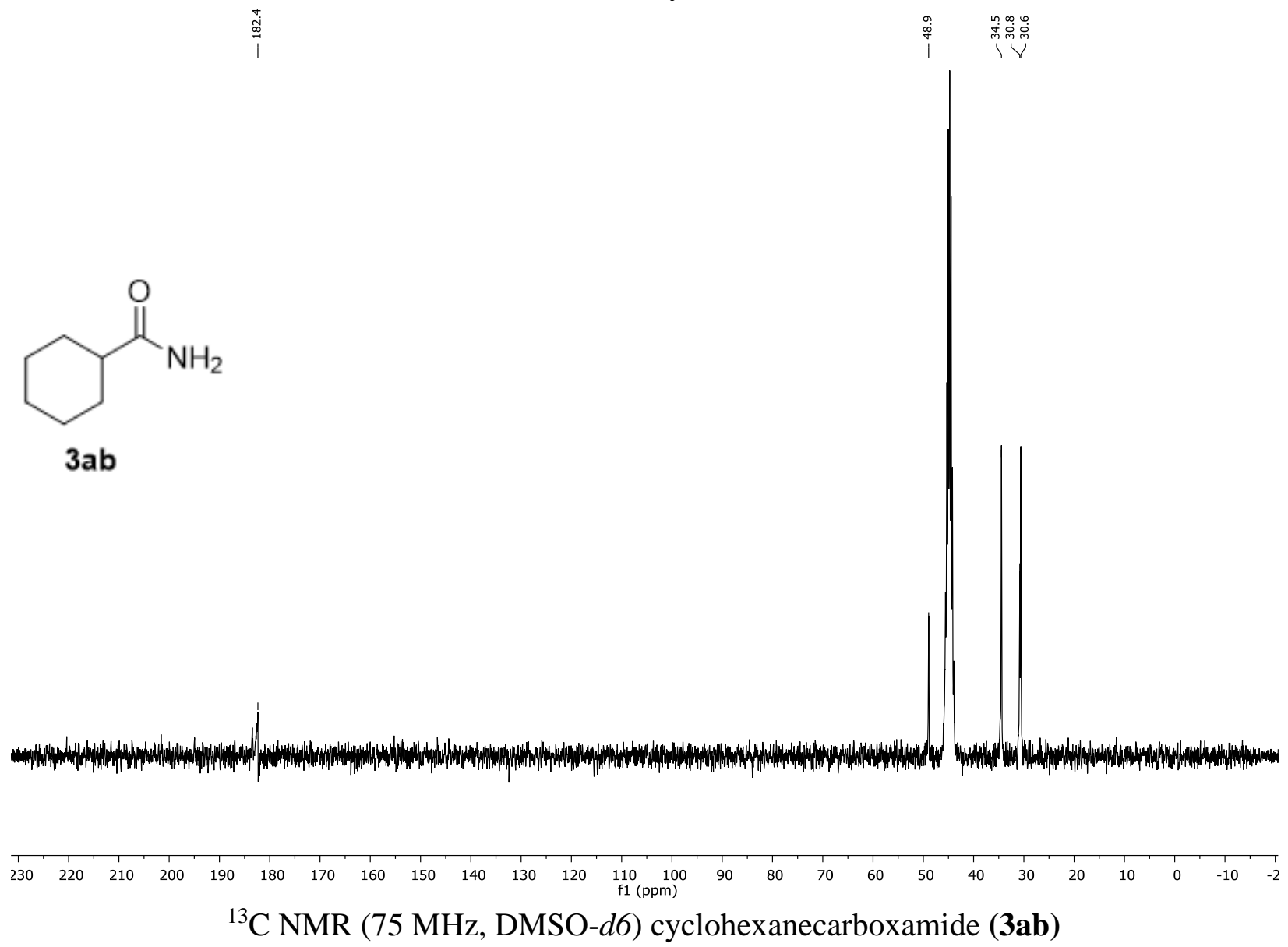




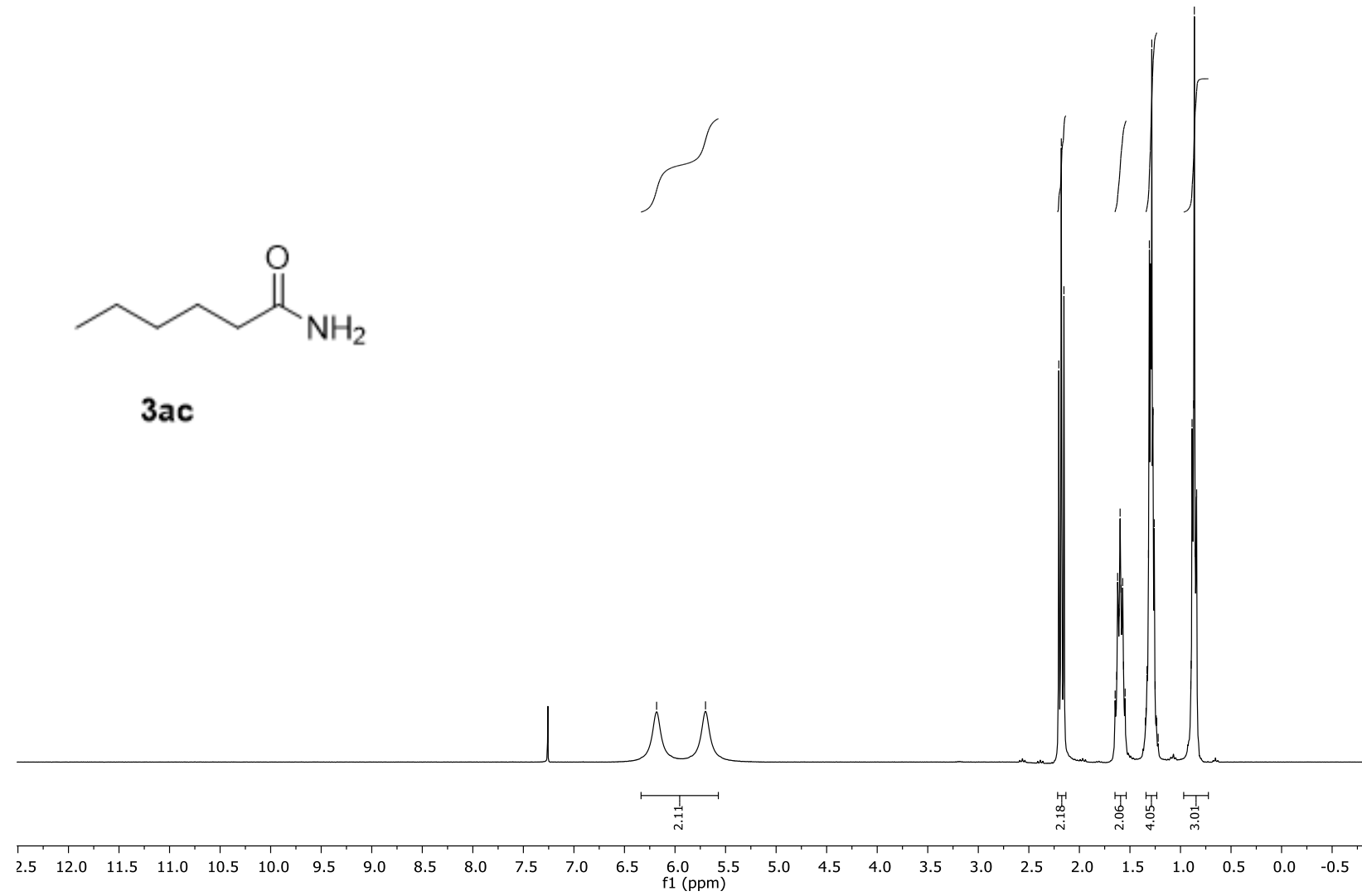

${ }^{1} \mathrm{H}$ NMR (300 MHz, Chloroform- $d$ ) hexanamide (3ac)

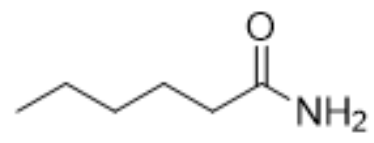

$3 a c$
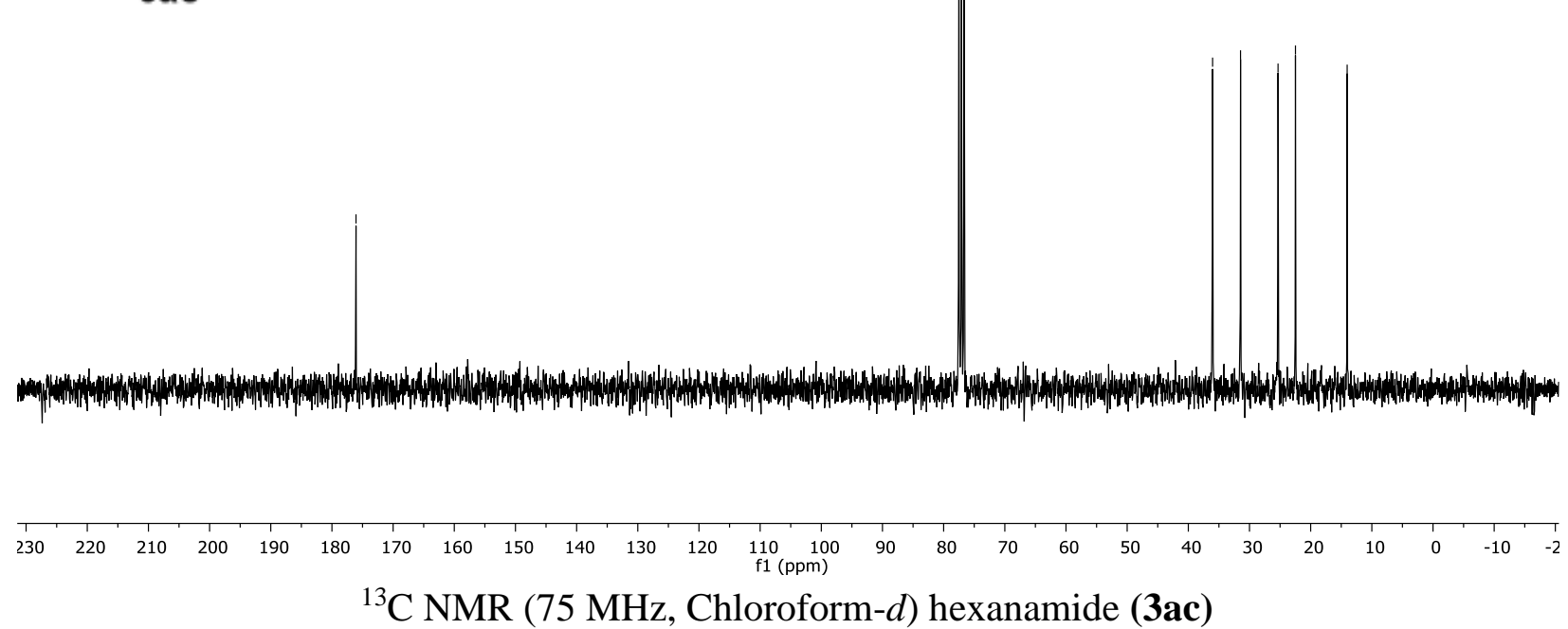


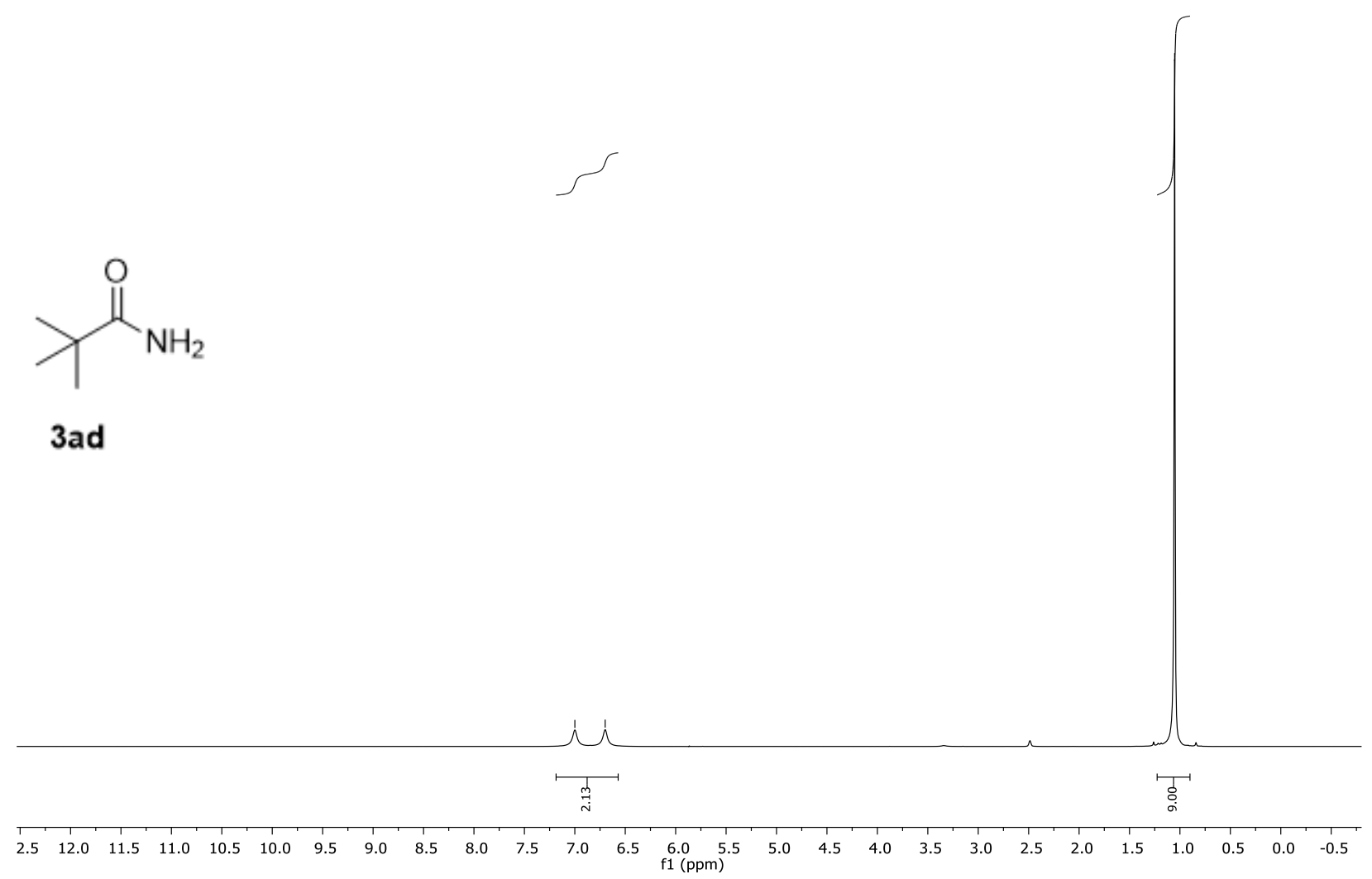

${ }^{1} \mathrm{H}$ NMR (300 MHz, DMSO- $d 6$ ) pivalamide (3ad)
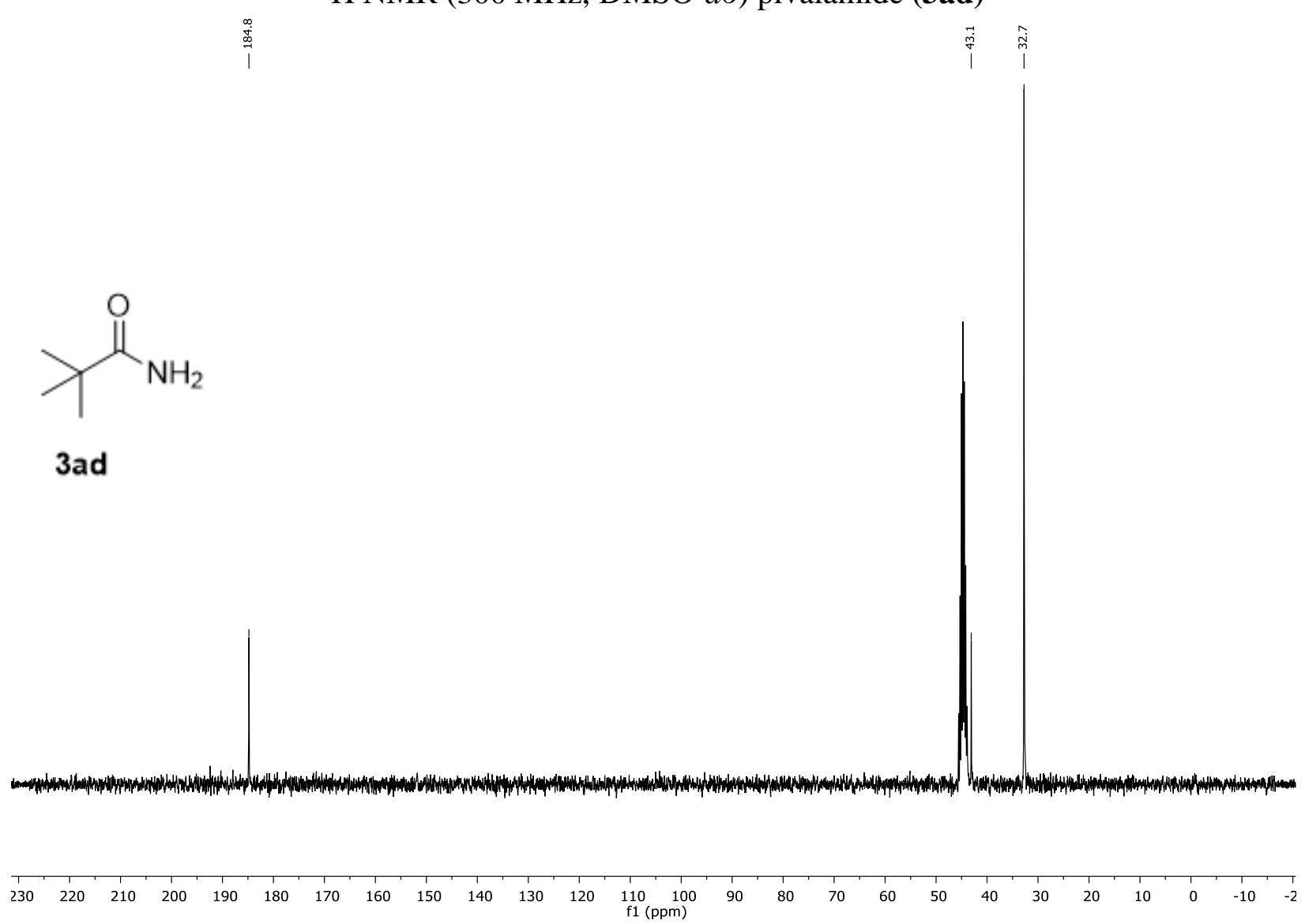

${ }^{13} \mathrm{C}$ NMR (75 MHz, DMSO- $d 6$ ) pivalamide (3ad) 


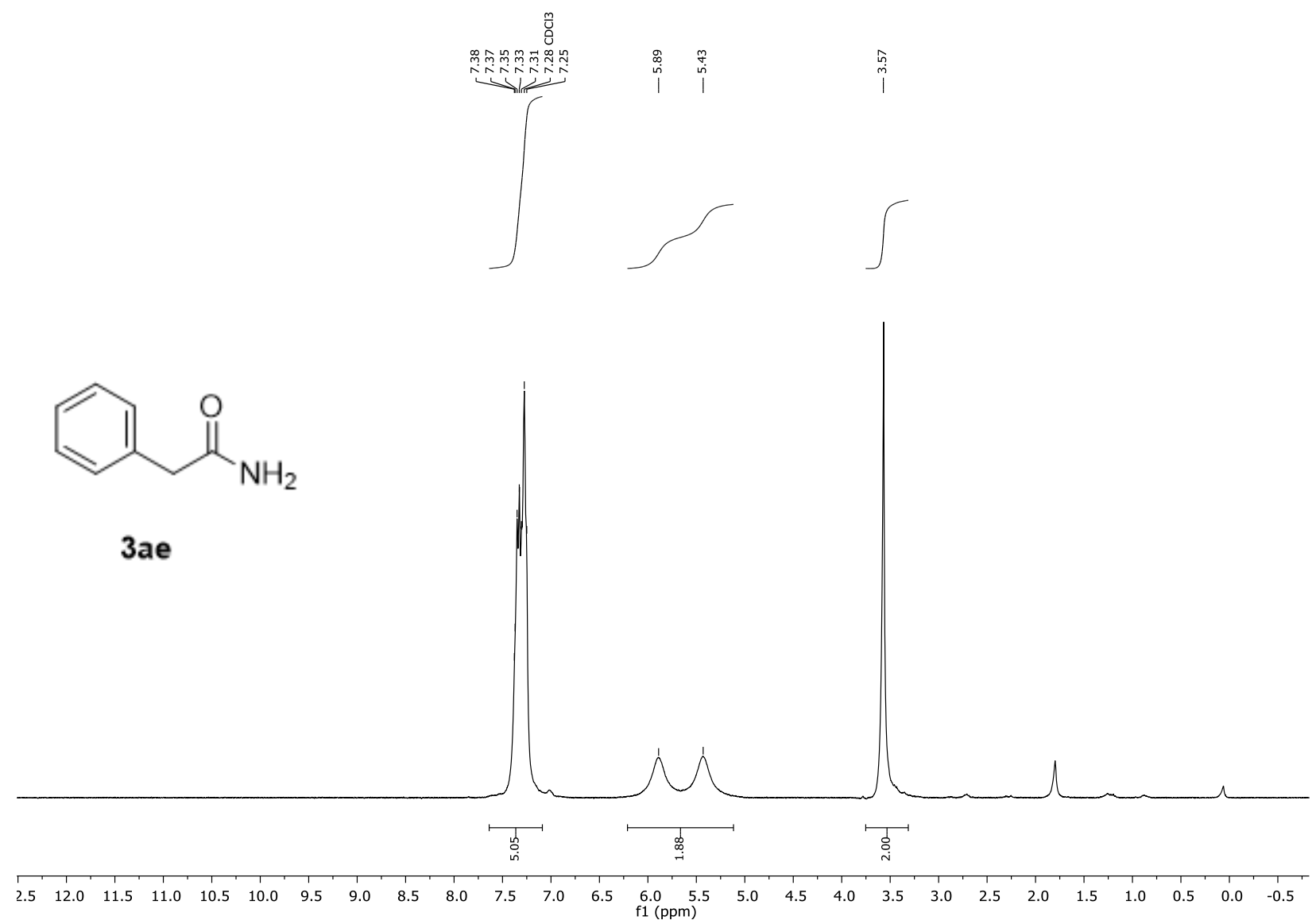

${ }^{1} \mathrm{H}$ NMR (300 MHz, DMSO-d6) 2-phenylacetamide (3ae)

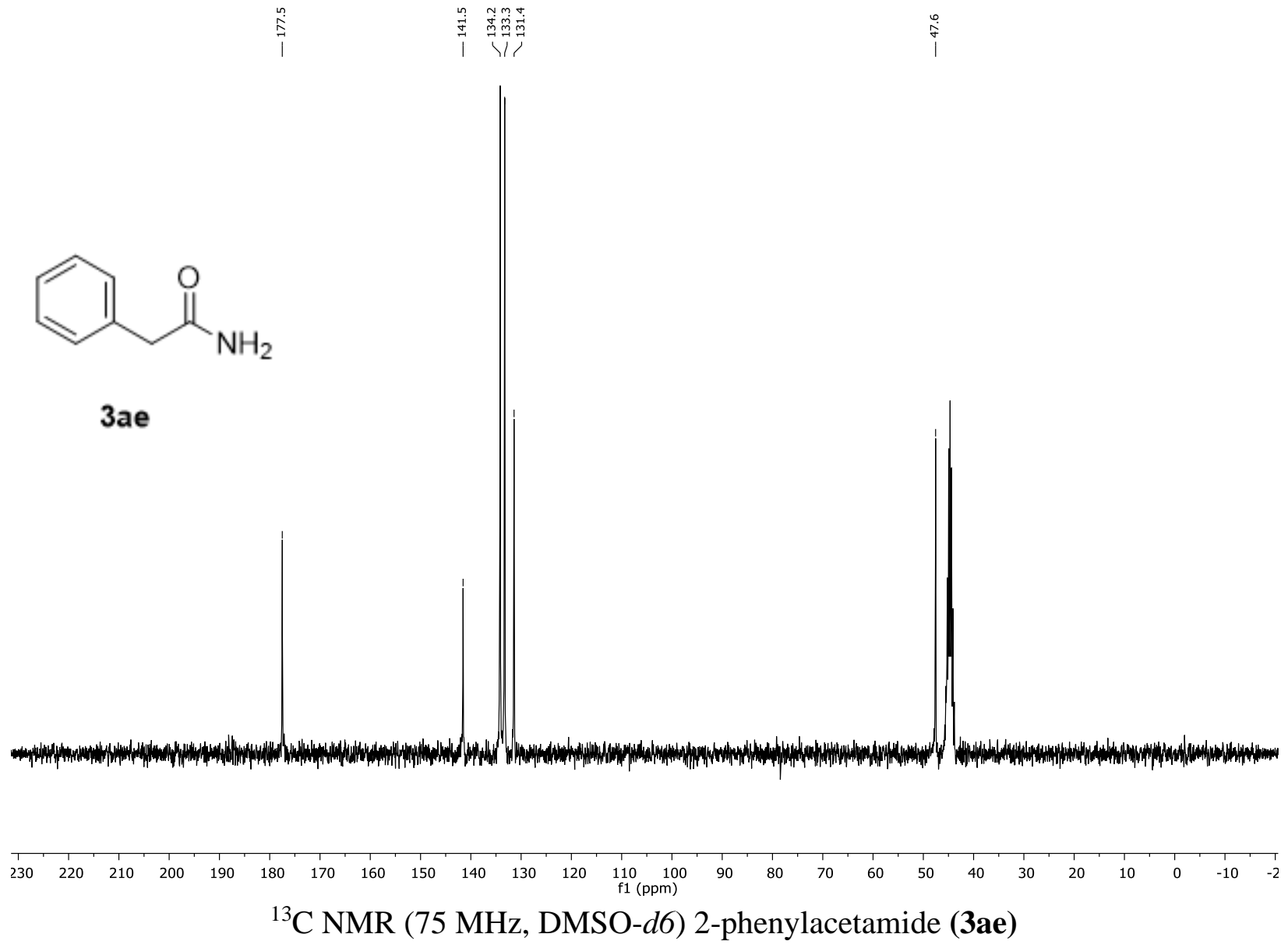



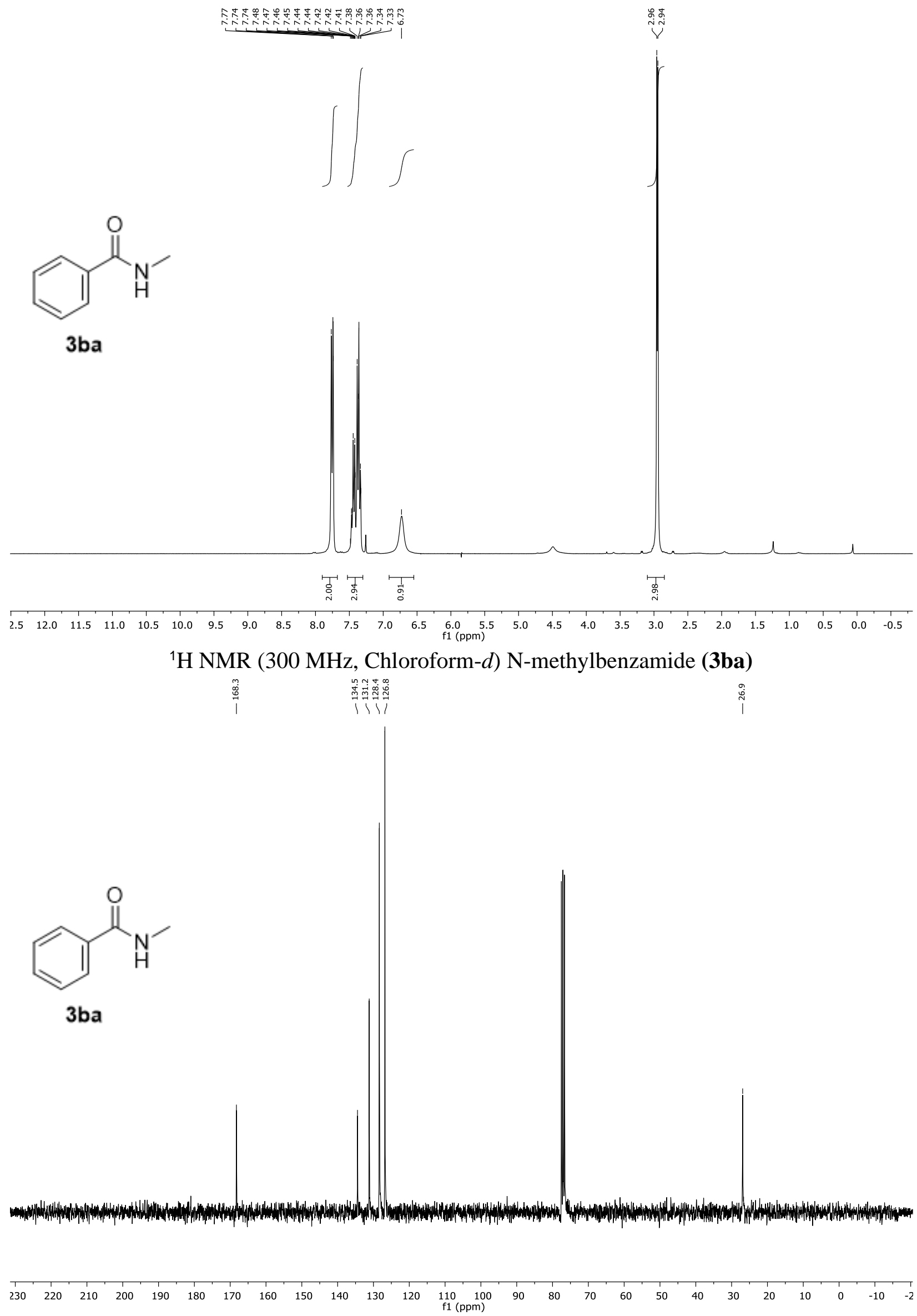

${ }^{13} \mathrm{C}$ NMR (75 MHz, Chloroform- $d$ ) N-methylbenzamide (3ba) 
<smiles>CNC(=O)C1CCCCC1</smiles>

$3 \mathrm{bb}$

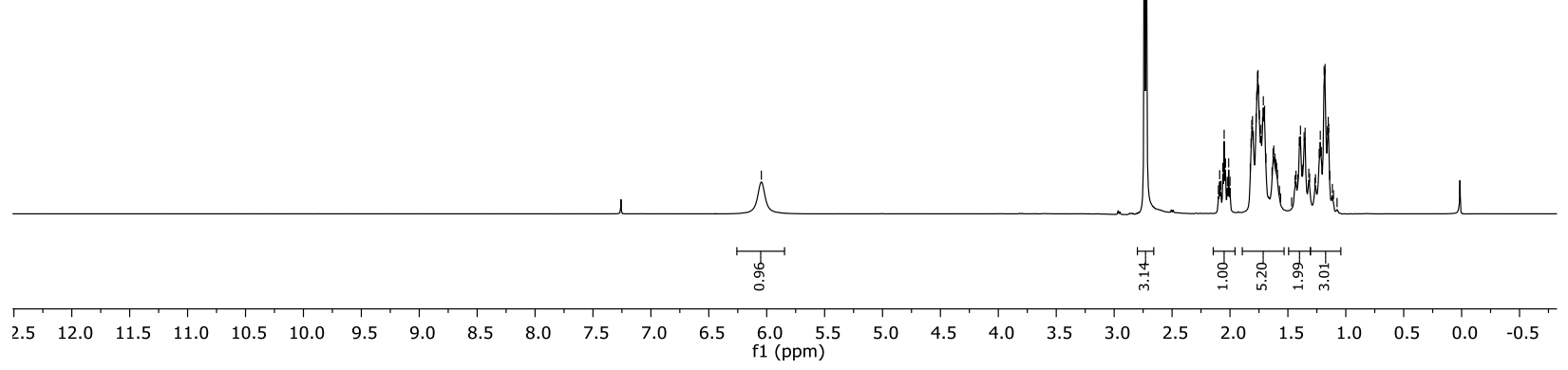

${ }^{1} \mathrm{H}$ NMR (300 MHz, Chloroform- $d$ ) N-methylcyclohexanecarboxamide (3bb)<smiles>CNC(=O)C1CCCCC1</smiles>

$3 \mathrm{bb}$

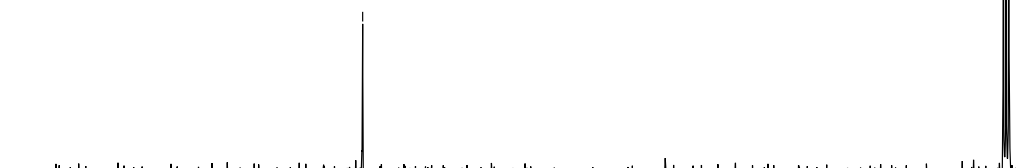

${ }^{13} \mathrm{C}$ NMR (75 MHz, Chloroform- $d$ ) N-methylcyclohexanecarboxamide (3bb) 


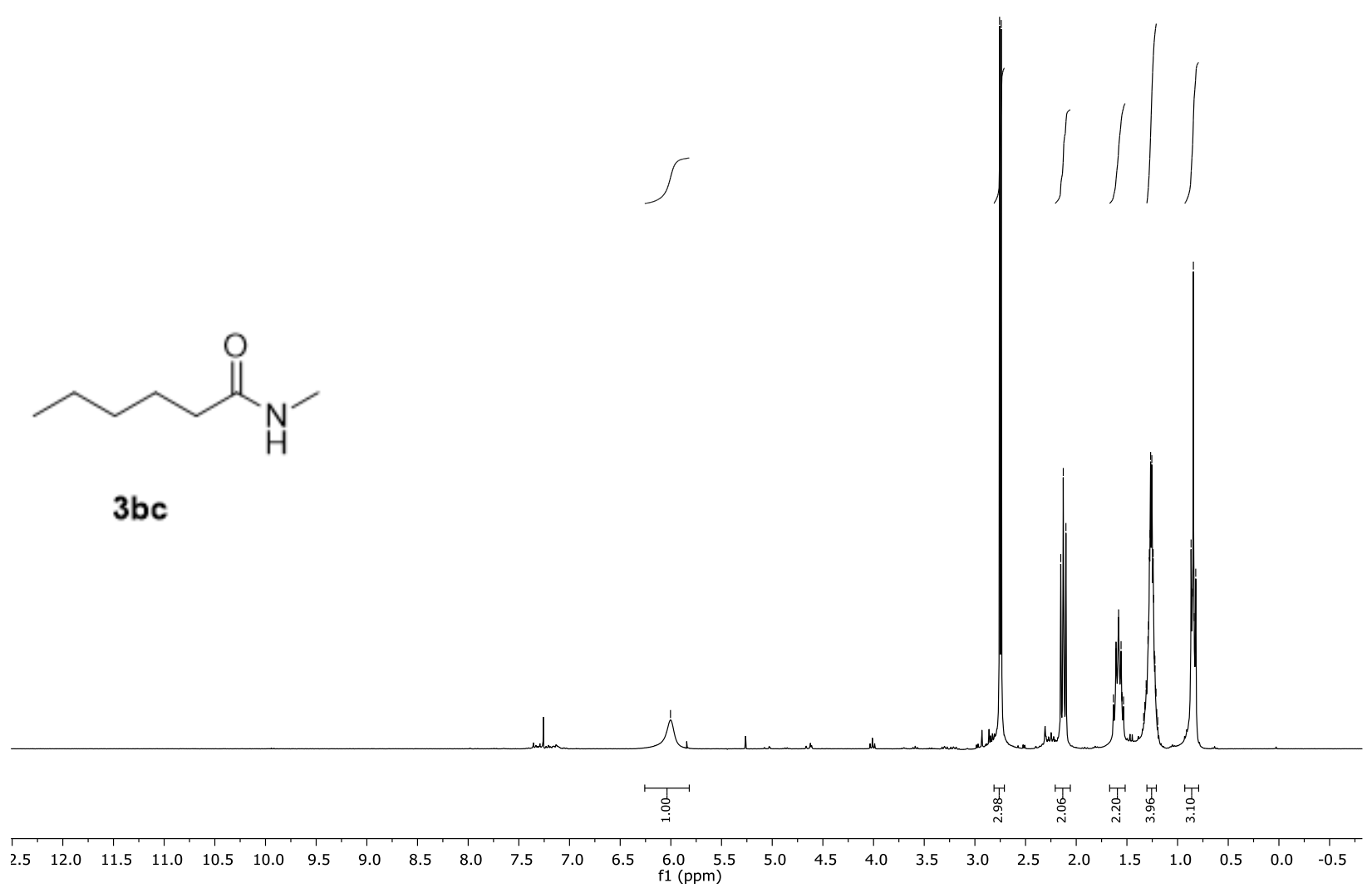

${ }^{1} \mathrm{H}$ NMR (300 MHz, Chloroform- $d$ ) N-methylhexanamide (3bc)

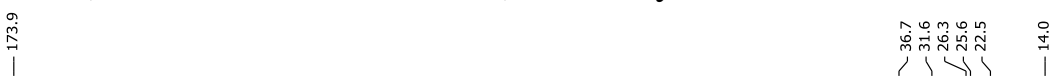<smiles>CCCCCC(=O)NC</smiles>

$3 \mathrm{bc}$

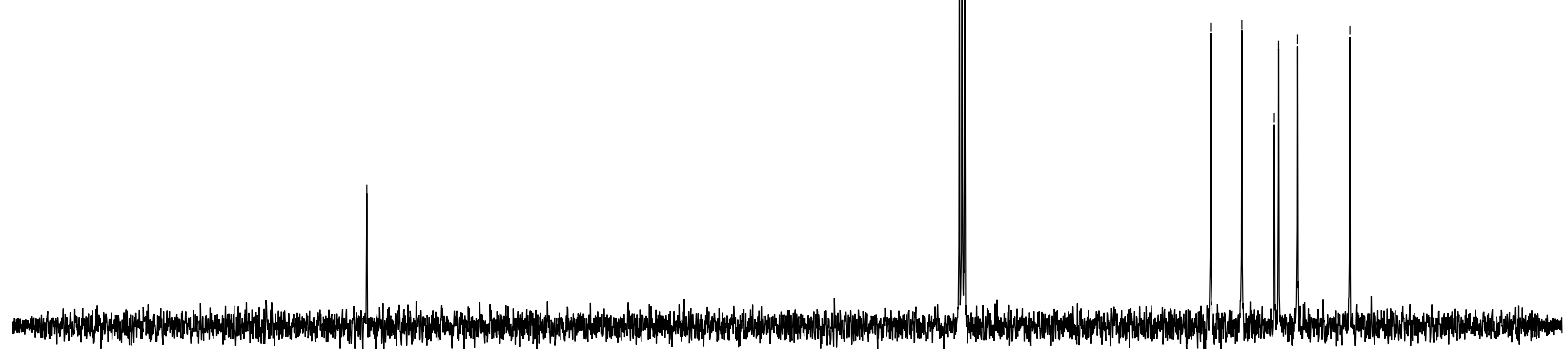

\footnotetext{
$\begin{array}{llllllllllllllllllllllllll}230 & 220 & 210 & 200 & 190 & 180 & 170 & 160 & 150 & 140 & 130 & 120 & \begin{array}{c}110 \\ \mathrm{f} 1(\mathrm{ppm})\end{array} & 90 & 80 & 70 & 60 & 50 & 40 & 30 & 20 & 10 & 0 & -10 & -2\end{array}$

${ }^{13} \mathrm{C}$ NMR (75 MHz, Chloroform- $d$ ) N-methylhexanamide (3bc)
} 


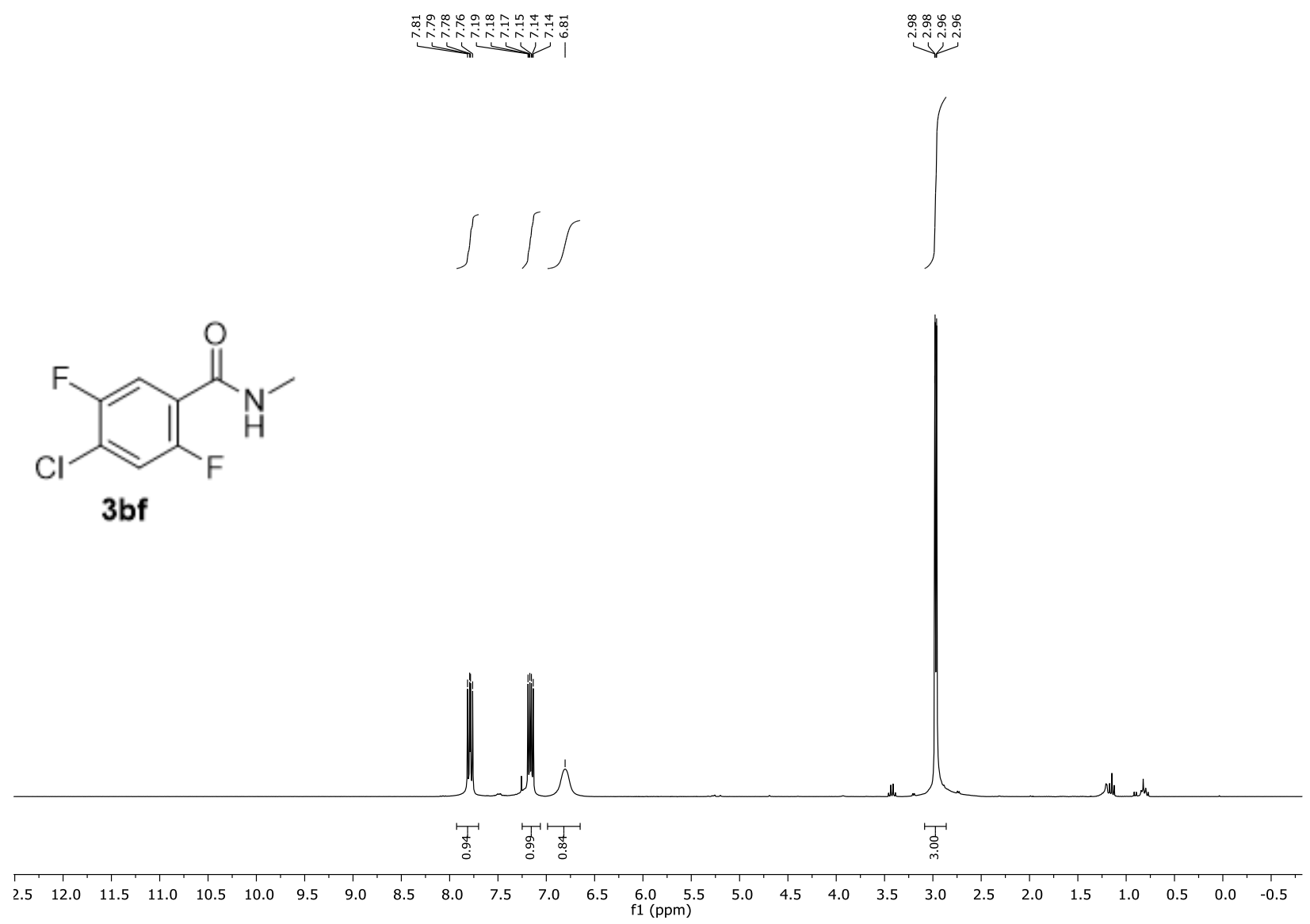

${ }^{1} \mathrm{H}$ NMR (300 MHz, Chloroform- $d$ ) 4-chloro-2,5-difluoro-N-methylbenzamide (3bf)

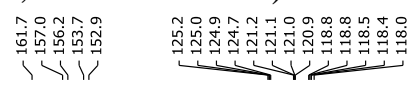<smiles>CNC(=O)c1cc(F)c(Cl)cc1F</smiles>
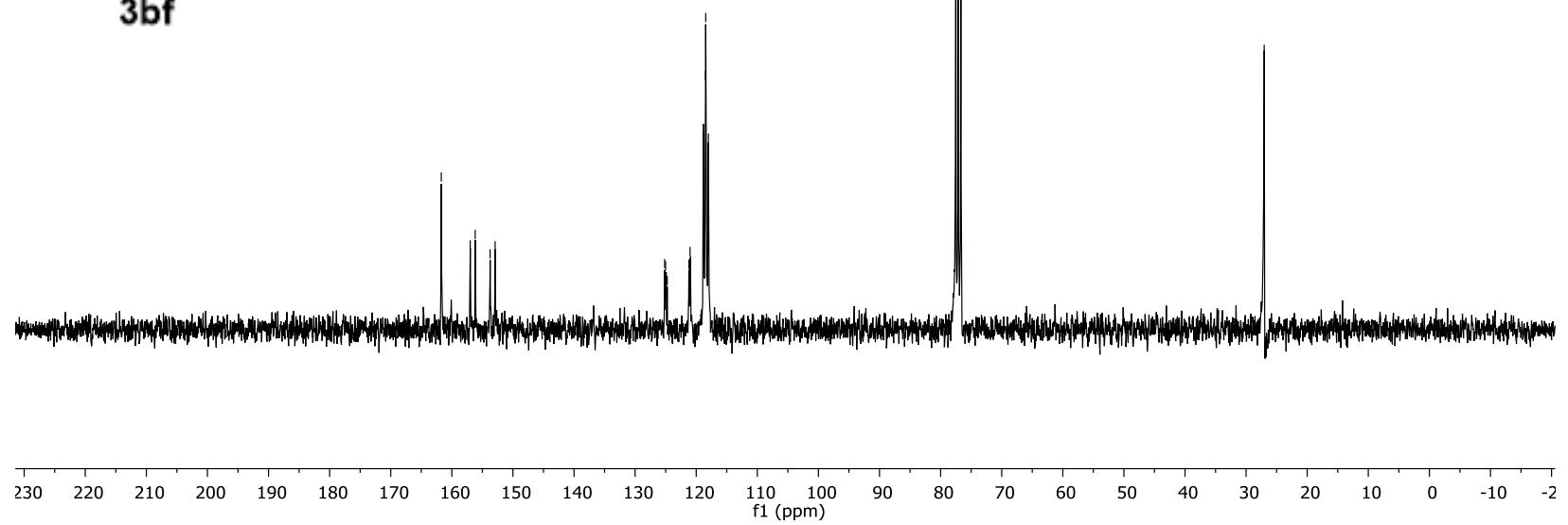

${ }^{13} \mathrm{C}$ NMR (75 MHz, Chloroform-d) 4-chloro-2,5-difluoro-N-methylbenzamide (3bf) 


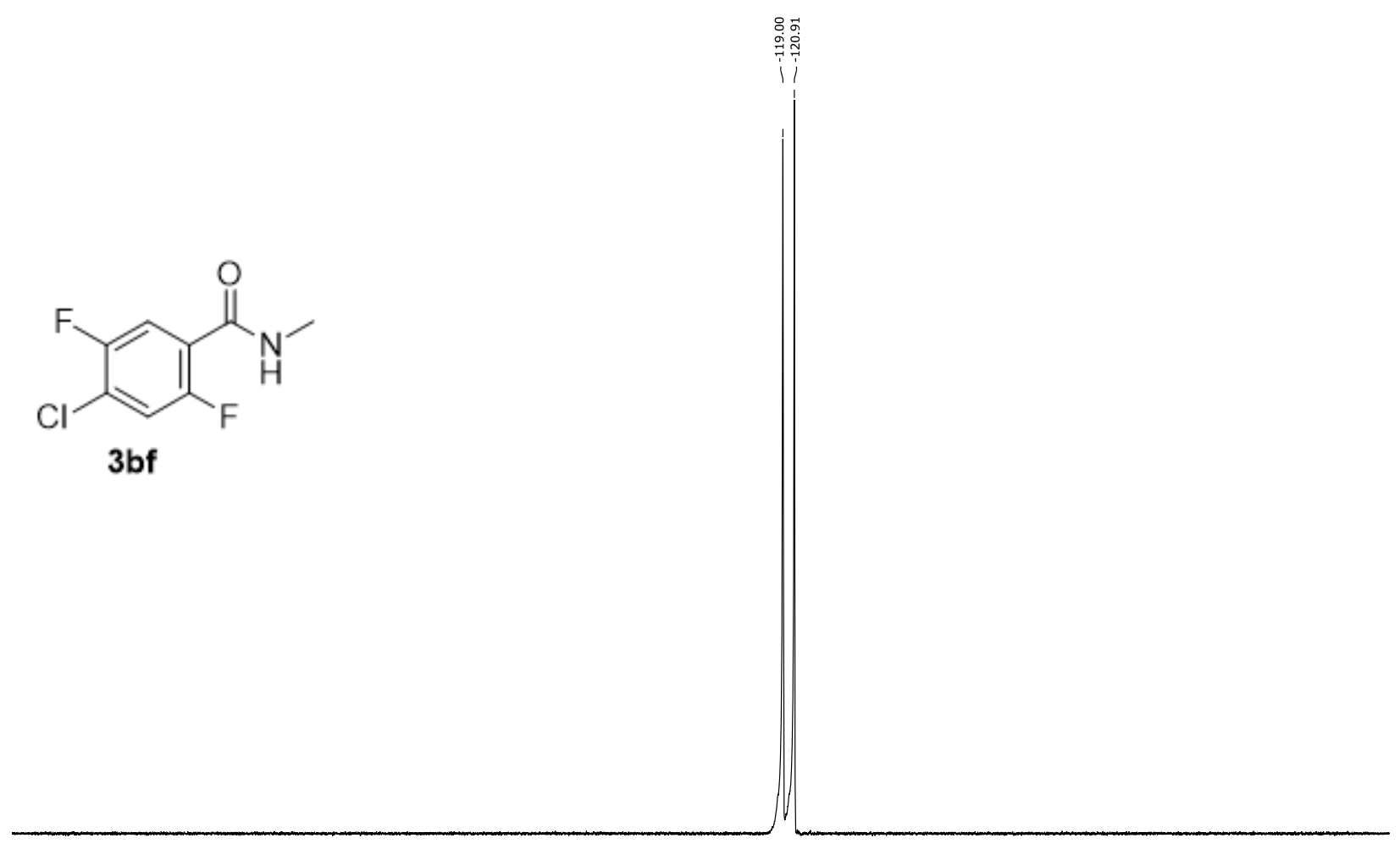

\begin{tabular}{|c|c|c|c|c|c|c|c|c|c|c|c|c|c|c|c|c|c|c|c|c|c|c|}
\hline 0 & -10 & -20 & -30 & -40 & -50 & -60 & -70 & -80 & -90 & $\begin{array}{c}-100 \\
f 1(\end{array}$ & $\begin{array}{l}-110 \\
\mathrm{pm})\end{array}$ & -120 & -130 & -140 & -150 & -160 & -170 & -180 & -190 & -200 & -210 & -2 \\
\hline
\end{tabular}

${ }^{19}$ F NMR (282 MHz, Chloroform- $d$ ) 4-chloro-2,5-difluoro-N-methylbenzamide (3bf)

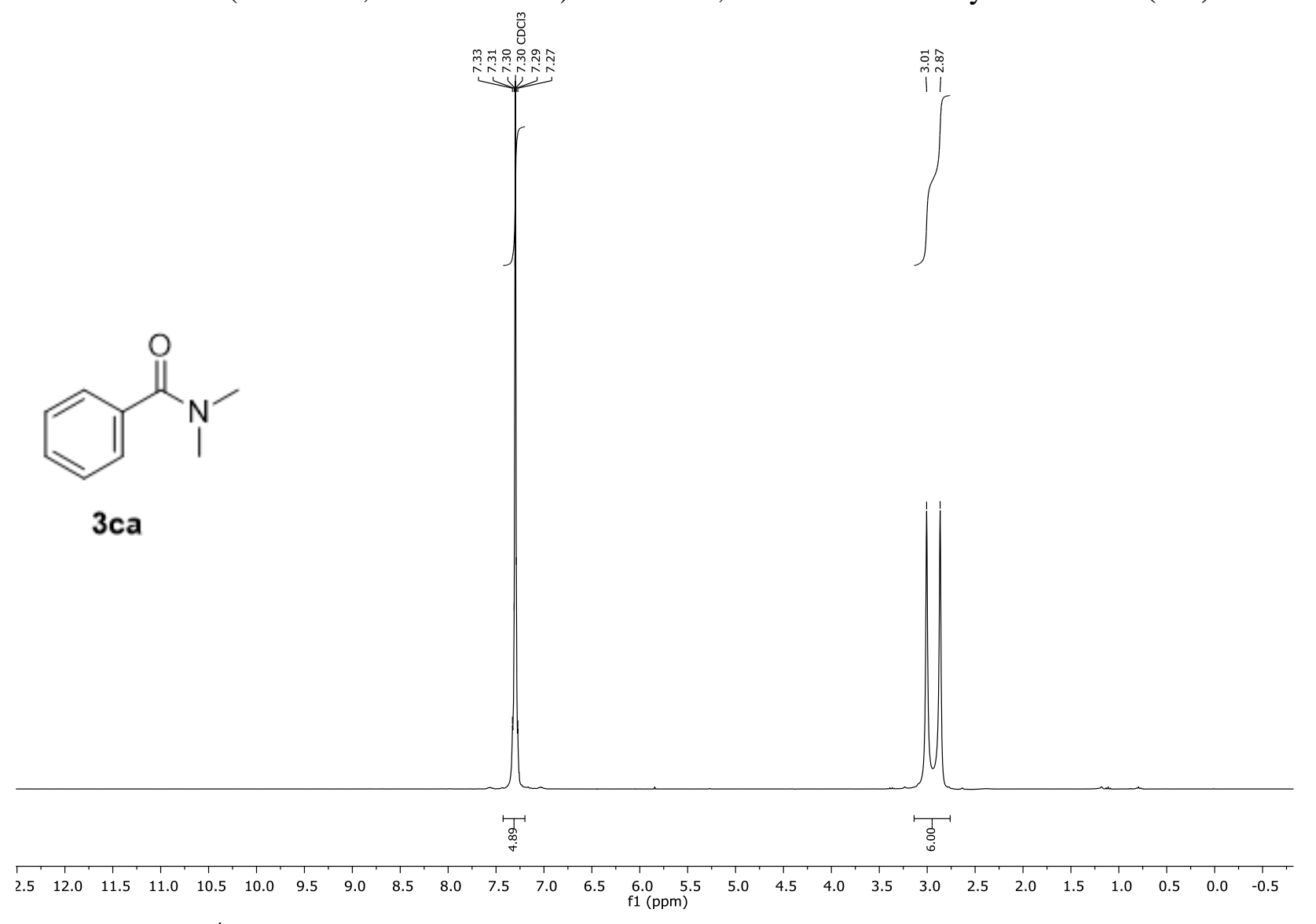

${ }^{1} \mathrm{H}$ NMR (300 MHz, Chloroform- $d$ ) N,N-dimethylbenzamide (3ca) 


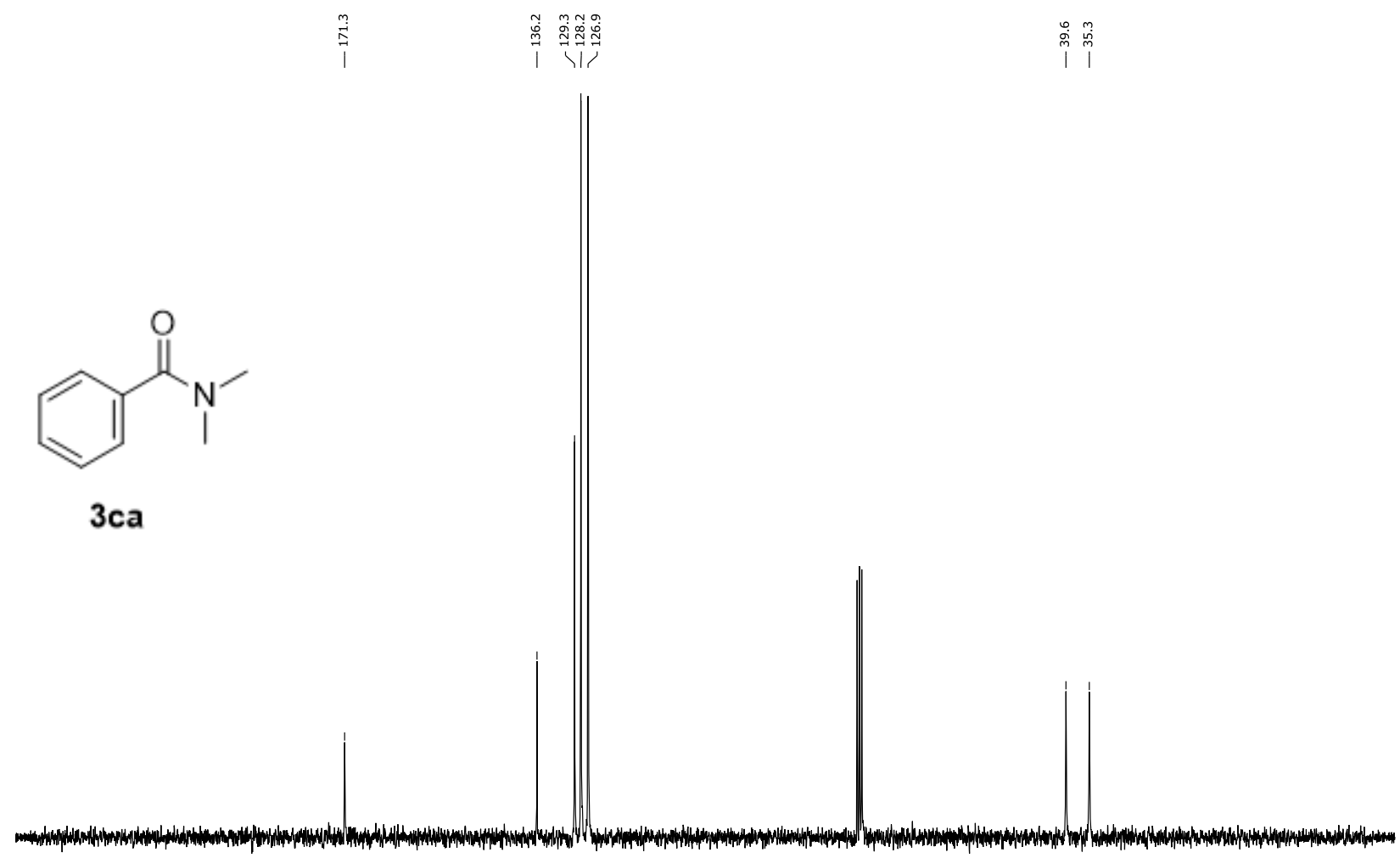

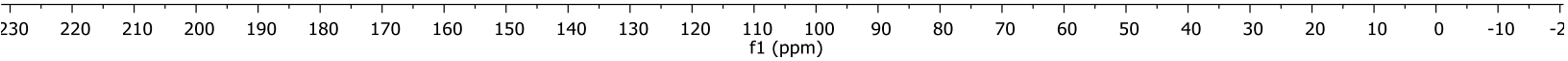

${ }^{13} \mathrm{C}$ NMR (75 MHz, Chloroform- $d$ ) N,N-dimethylbenzamide (3ca)

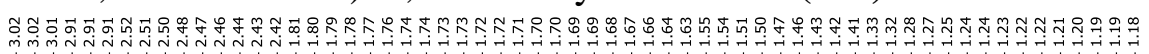

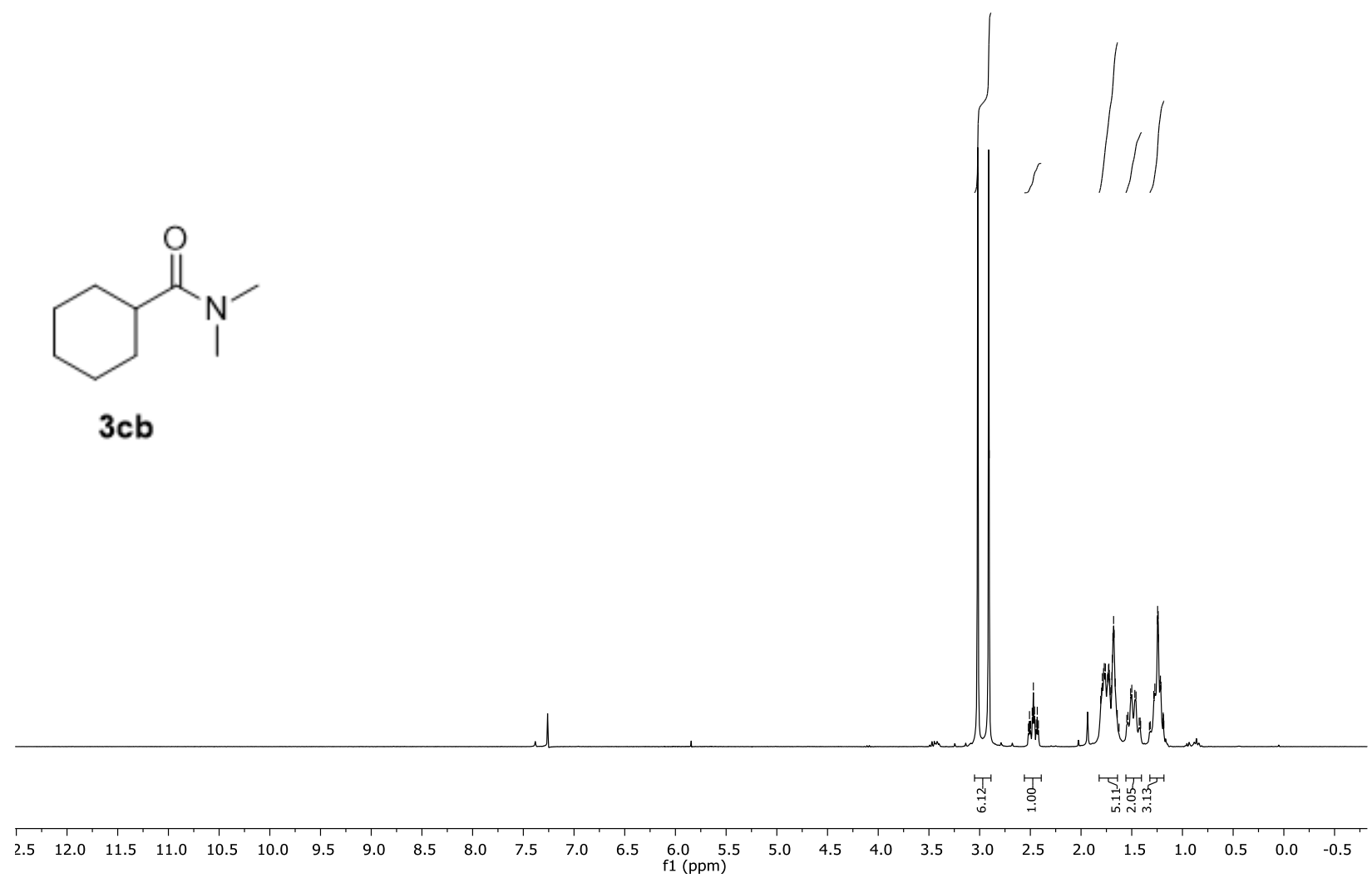

${ }^{1} \mathrm{H}$ NMR (300 MHz, Chloroform- $d$ ) N,N-dimethylcyclohexanecarboxamide (3cb) 
<smiles>CN(C)C(=O)C1CCCCC1</smiles>
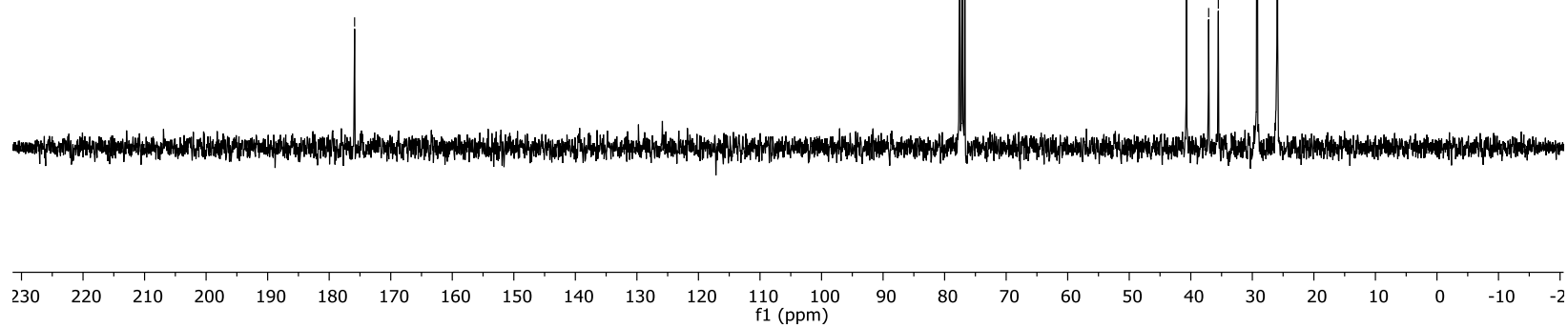

${ }^{13} \mathrm{C}$ NMR (75 MHz, Chloroform- $d$ ) N,N-dimethylcyclohexanecarboxamide (3cb)

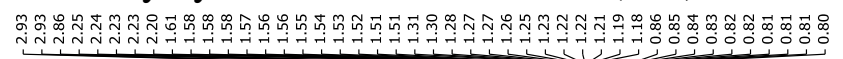

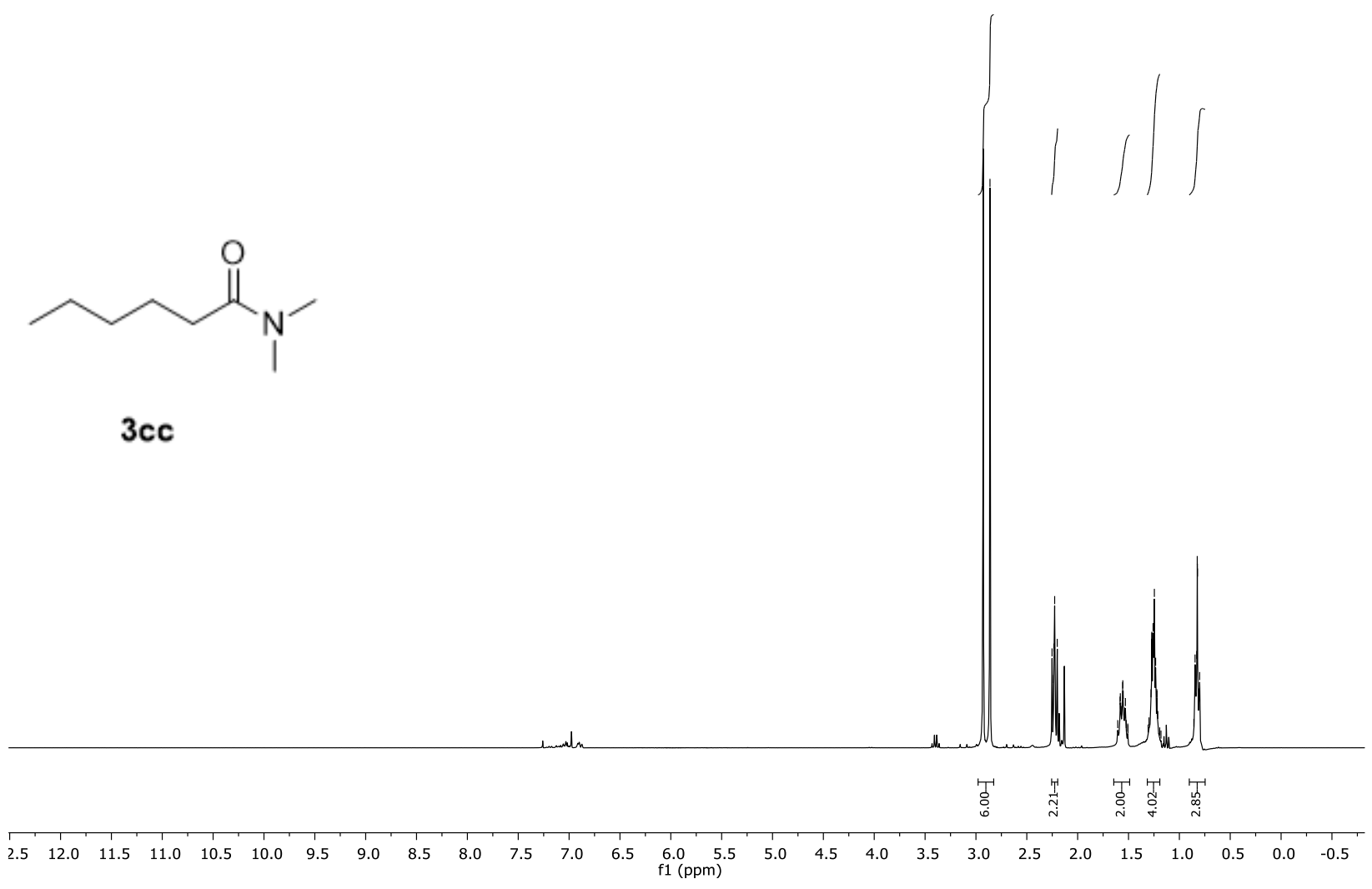

${ }^{1} \mathrm{H}$ NMR (300 MHz, Chloroform- $d$ ) N,N-dimethylhexanamide (3cc) 


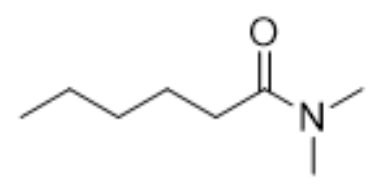

$3 \mathrm{cc}$

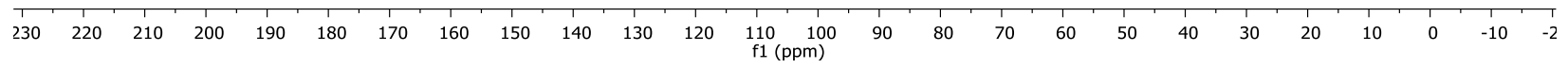

${ }^{13} \mathrm{C}$ NMR (75 MHz, Chloroform- $d$ ) N,N-dimethylhexanamide (3cc)

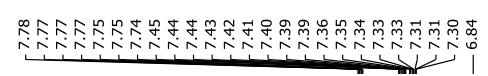
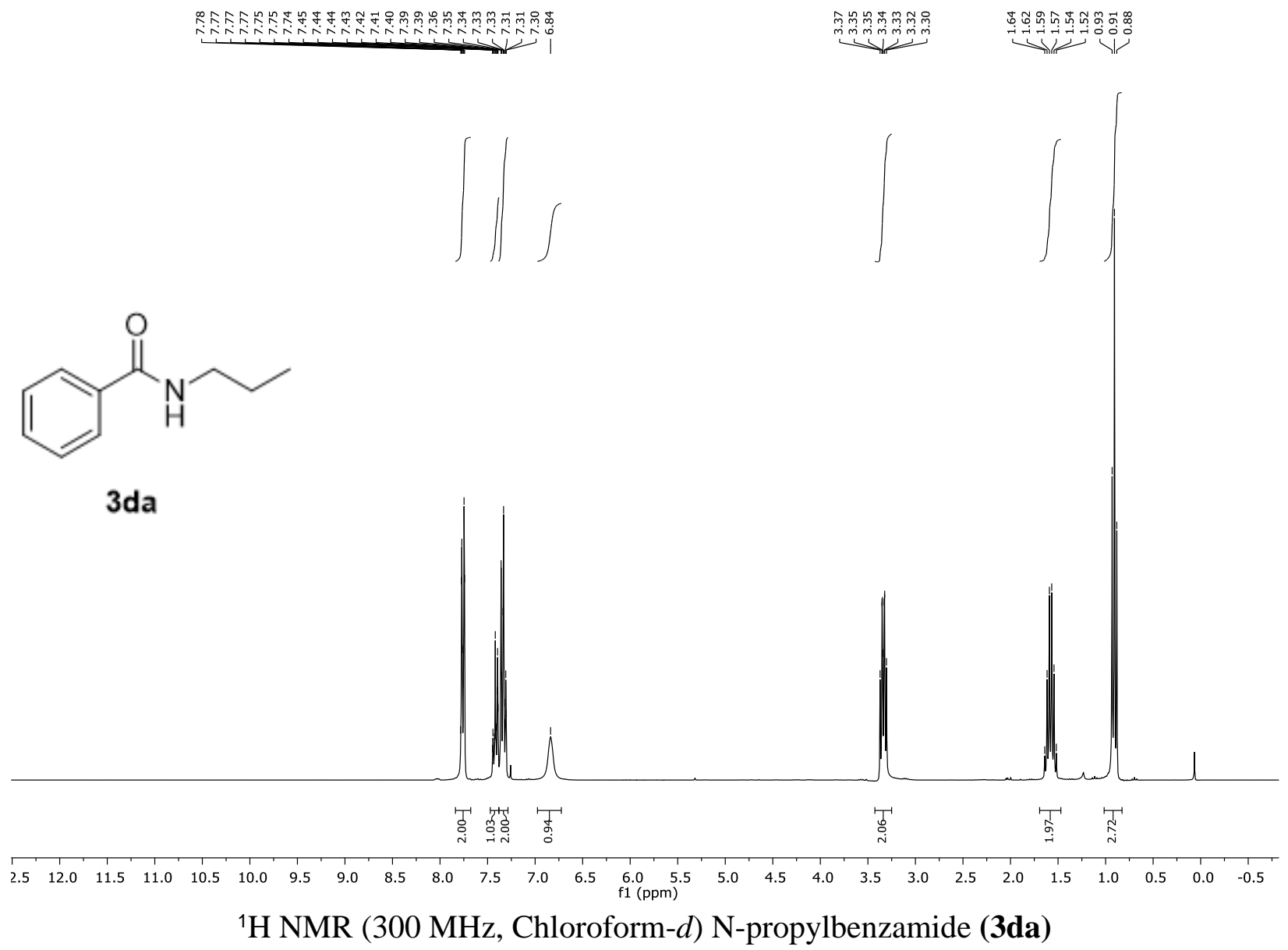

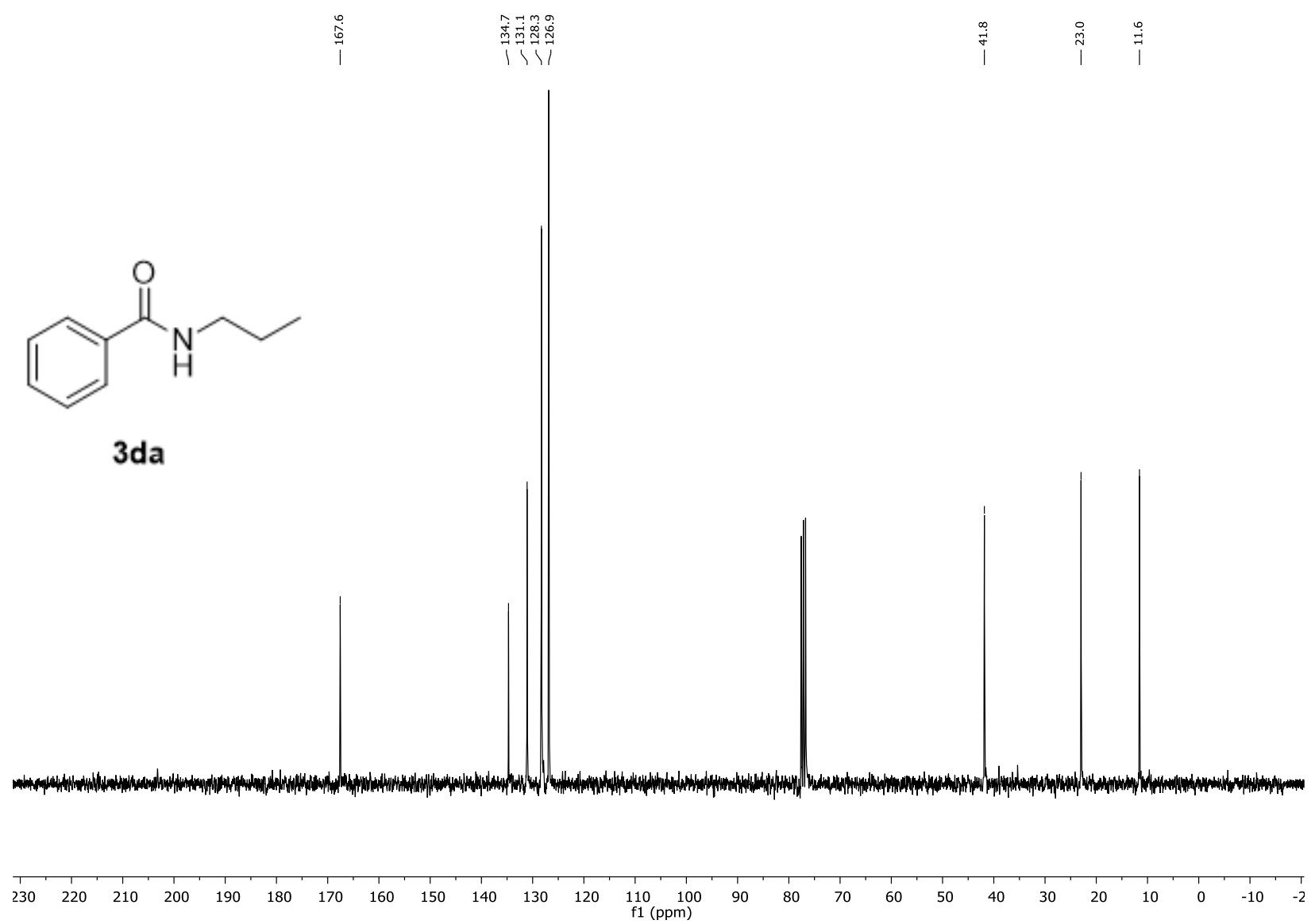

${ }^{13} \mathrm{C}$ NMR (75 MHz, Chloroform- $d$ ) N-propylbenzamide (3da)

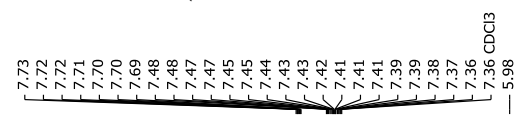<smiles>CC(C)(C)NC(=O)c1ccccc1</smiles>

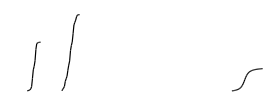

3ea

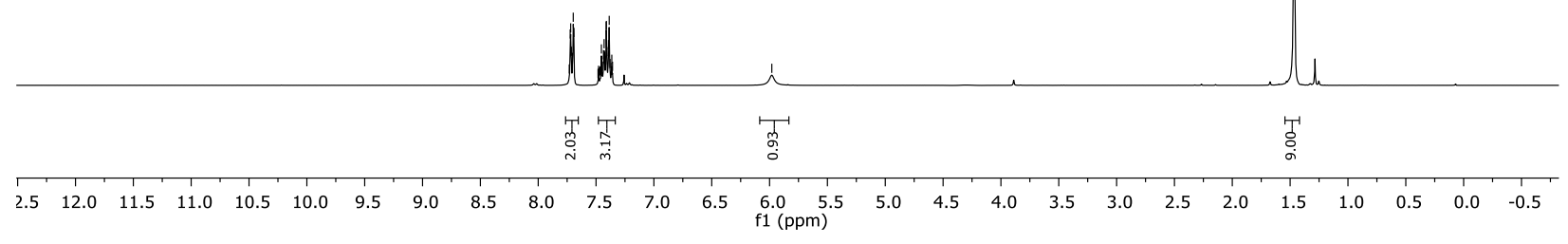

${ }^{1} \mathrm{H}$ NMR (300 MHz, Chloroform- $d$ ) N-(tert-butyl)benzamide (3ea) 


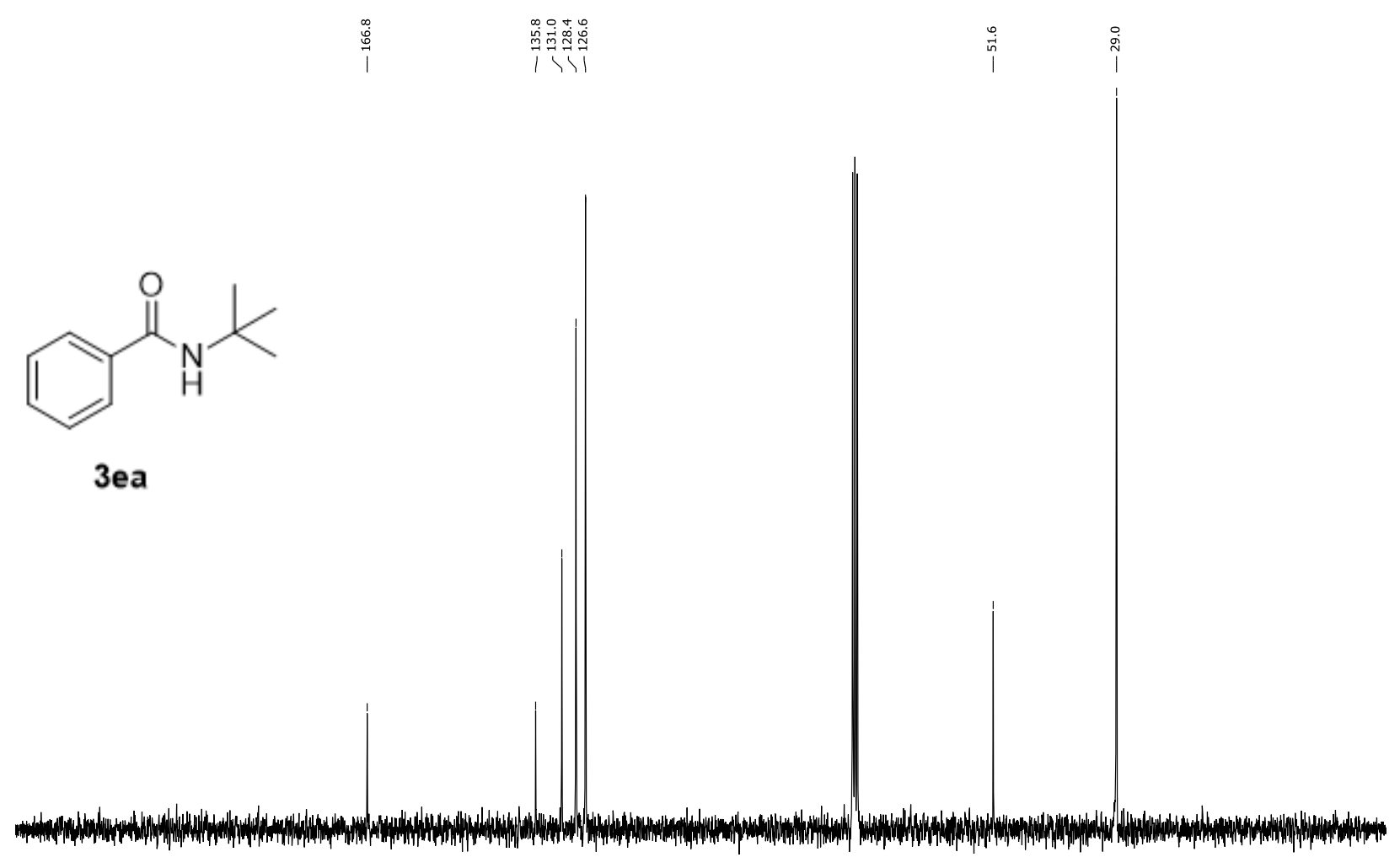

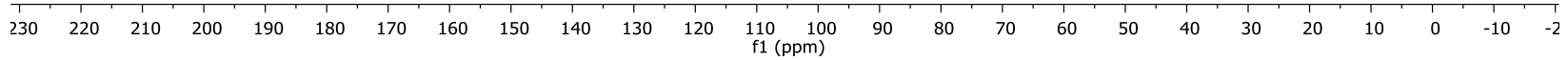

${ }^{13} \mathrm{C}$ NMR (75 MHz, Chloroform- $d$ ) N-(tert-butyl)benzamide (3ea)

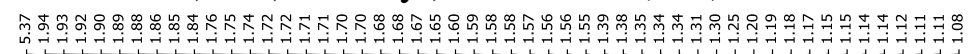

$\int$

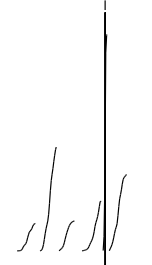

$\mathrm{C}_{\mathrm{H}}^{\mathrm{O}}$

$3 e b$

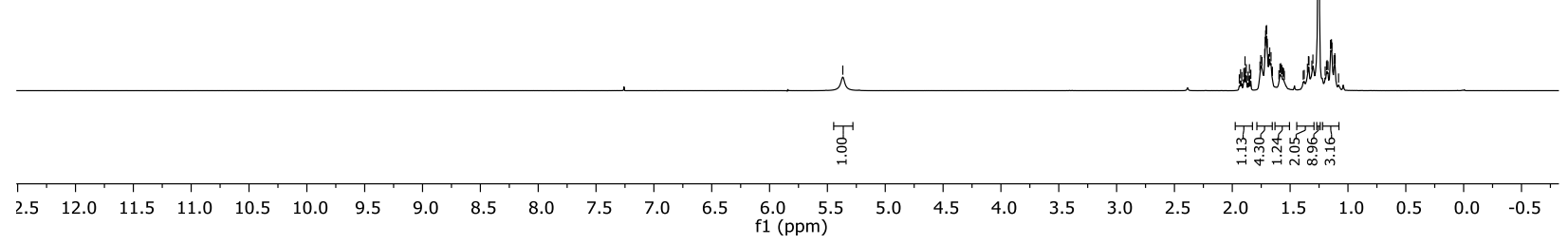

${ }^{1} \mathrm{H}$ NMR (300 MHz, Chloroform- $d$ ) N-(tert-butyl)cyclohexanecarboxamide (3eb) 


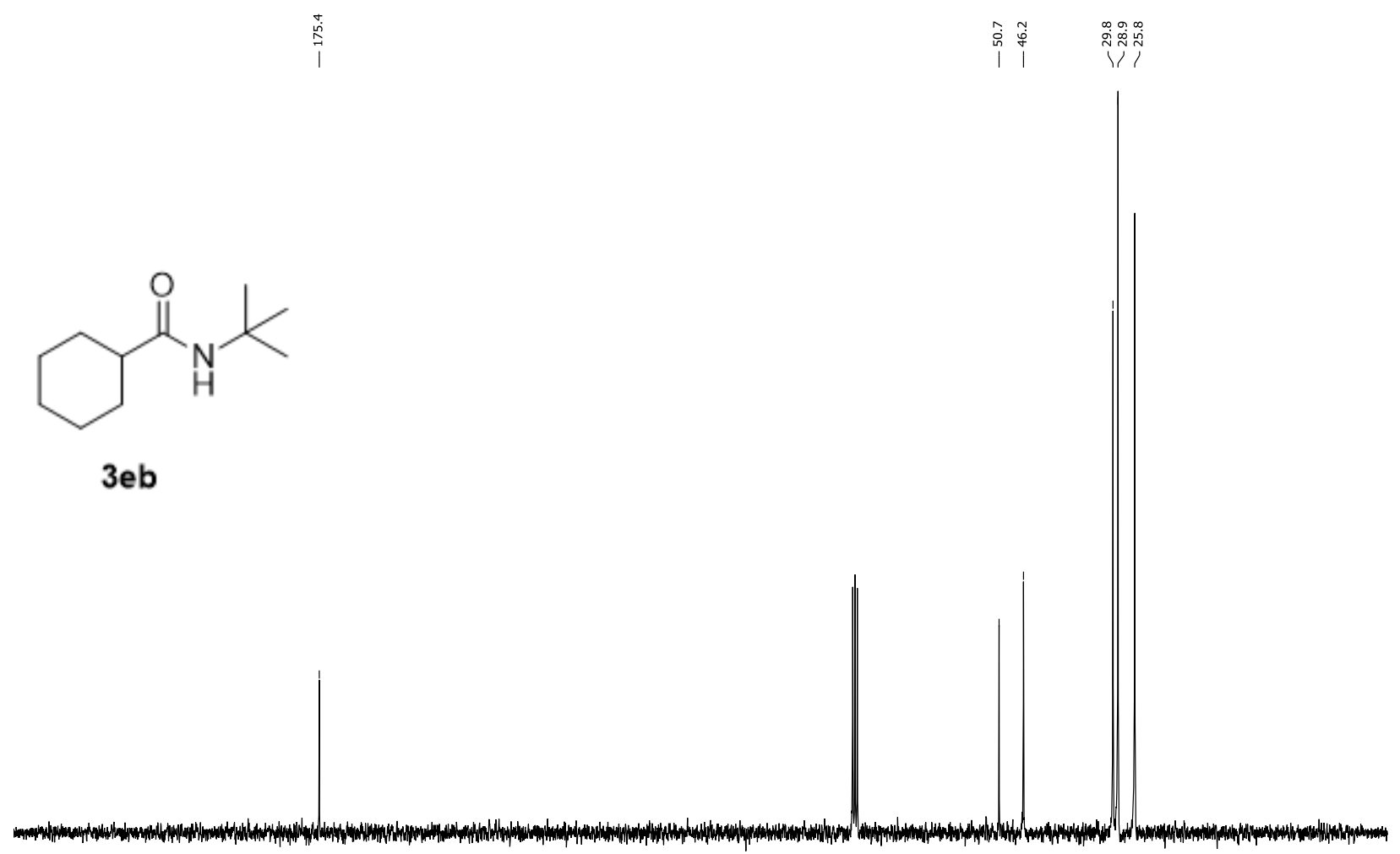

$\begin{array}{llllllllllllllllllllllllll}1 & 230 & 220 & 210 & 200 & 190 & 180 & 170 & 160 & 150 & 140 & 130 & 120 & \begin{array}{c}110 \\ \mathrm{f} 1(\mathrm{ppm})\end{array} & 100 & 80 & 70 & 60 & 50 & 40 & 30 & 20 & 10 & 0 & -10 & -2\end{array}$

${ }^{13} \mathrm{C}$ NMR (75 MHz, Chloroform- $d$ ) N-(tert-butyl)cyclohexanecarboxamide (3eb)

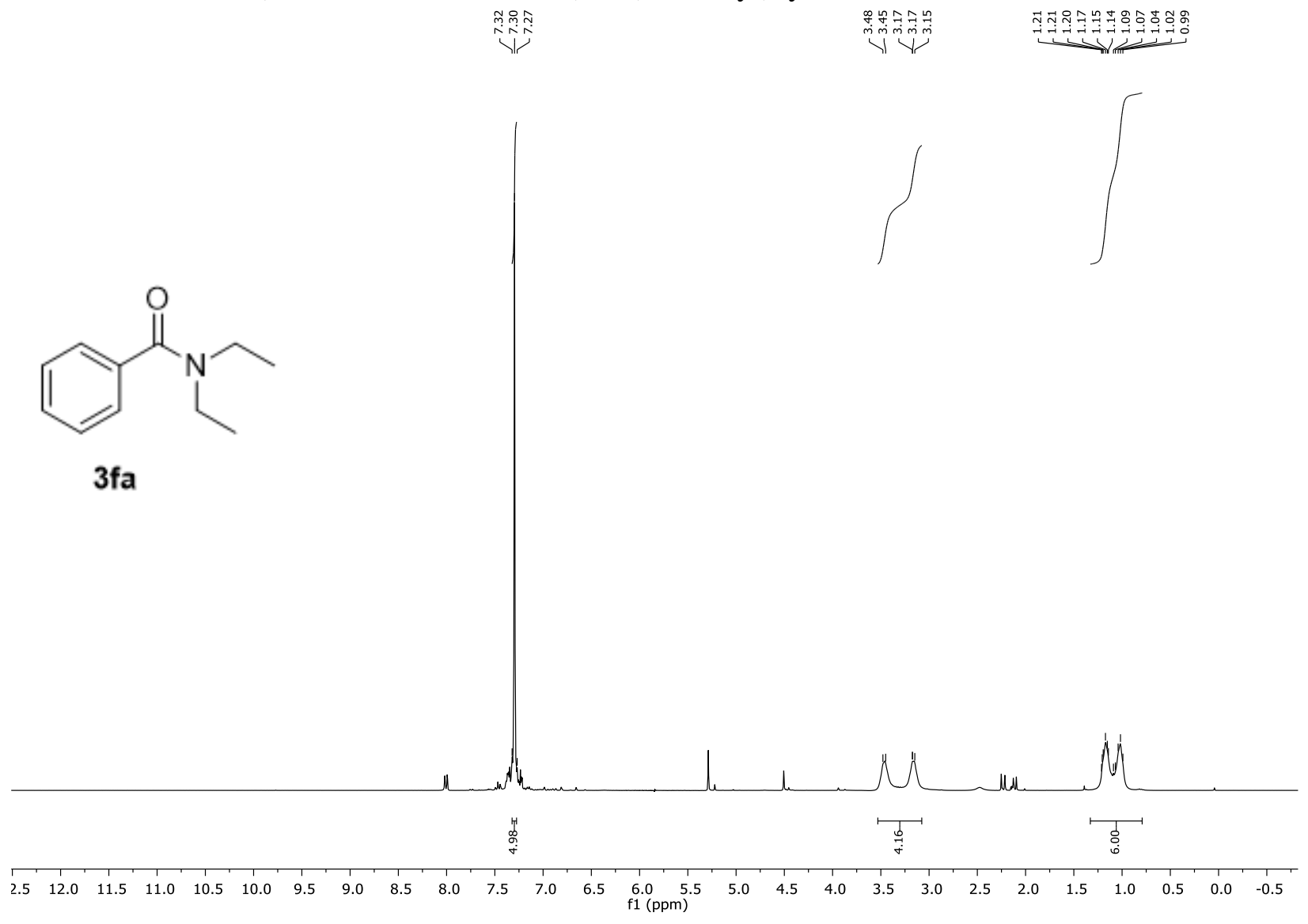

${ }^{1} \mathrm{H}$ NMR (300 MHz, Chloroform- $d$ ) N,N-diethylbenzamide (3fa) 

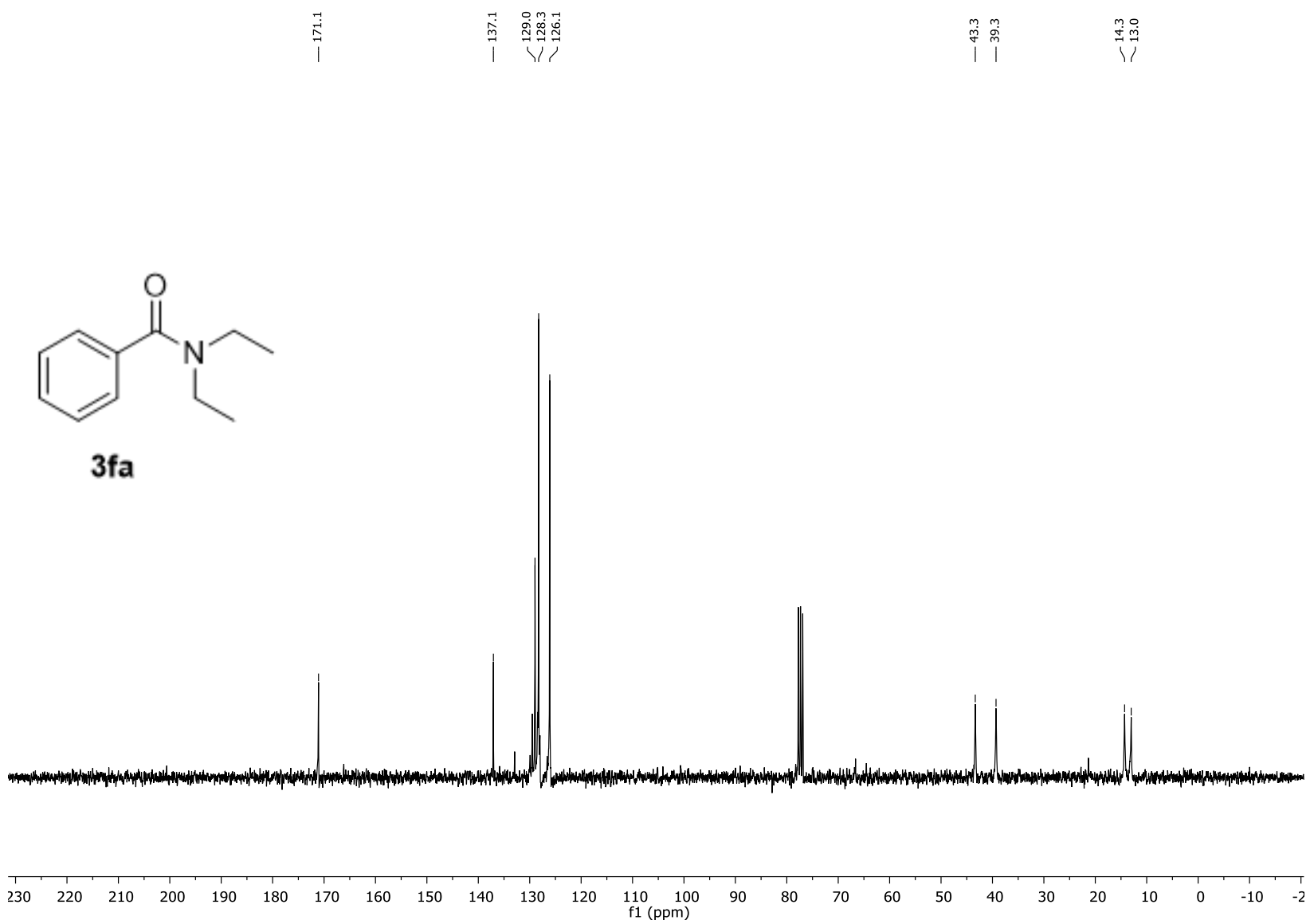

${ }^{13} \mathrm{C}$ NMR (75 MHz, Chloroform- $d$ ) N,N-diethylbenzamide (3fa)

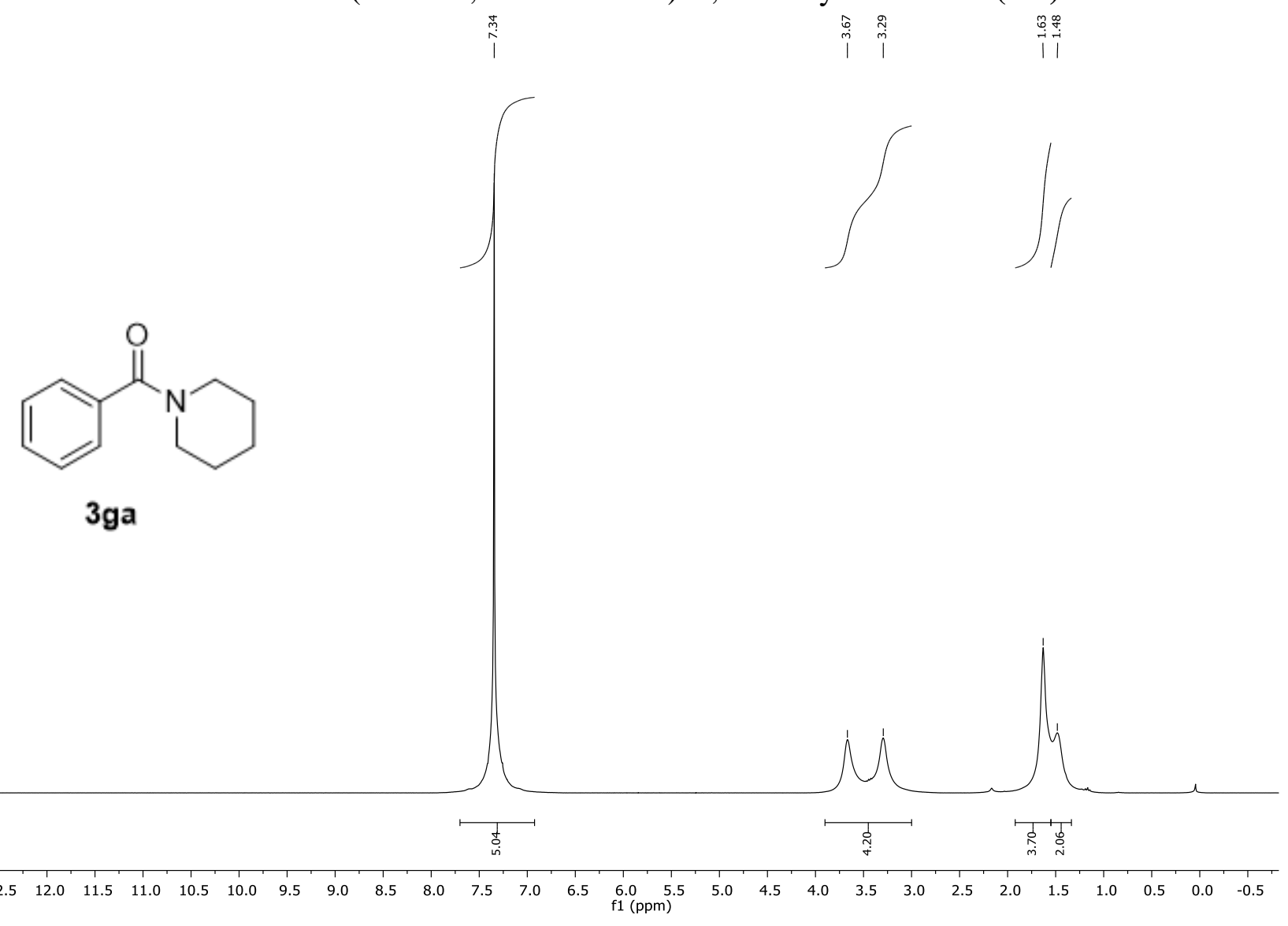

${ }^{1} \mathrm{H}$ NMR (300 MHz, Chloroform- $d$ ) phenyl(piperidin-1-yl)methanone (3ga) 


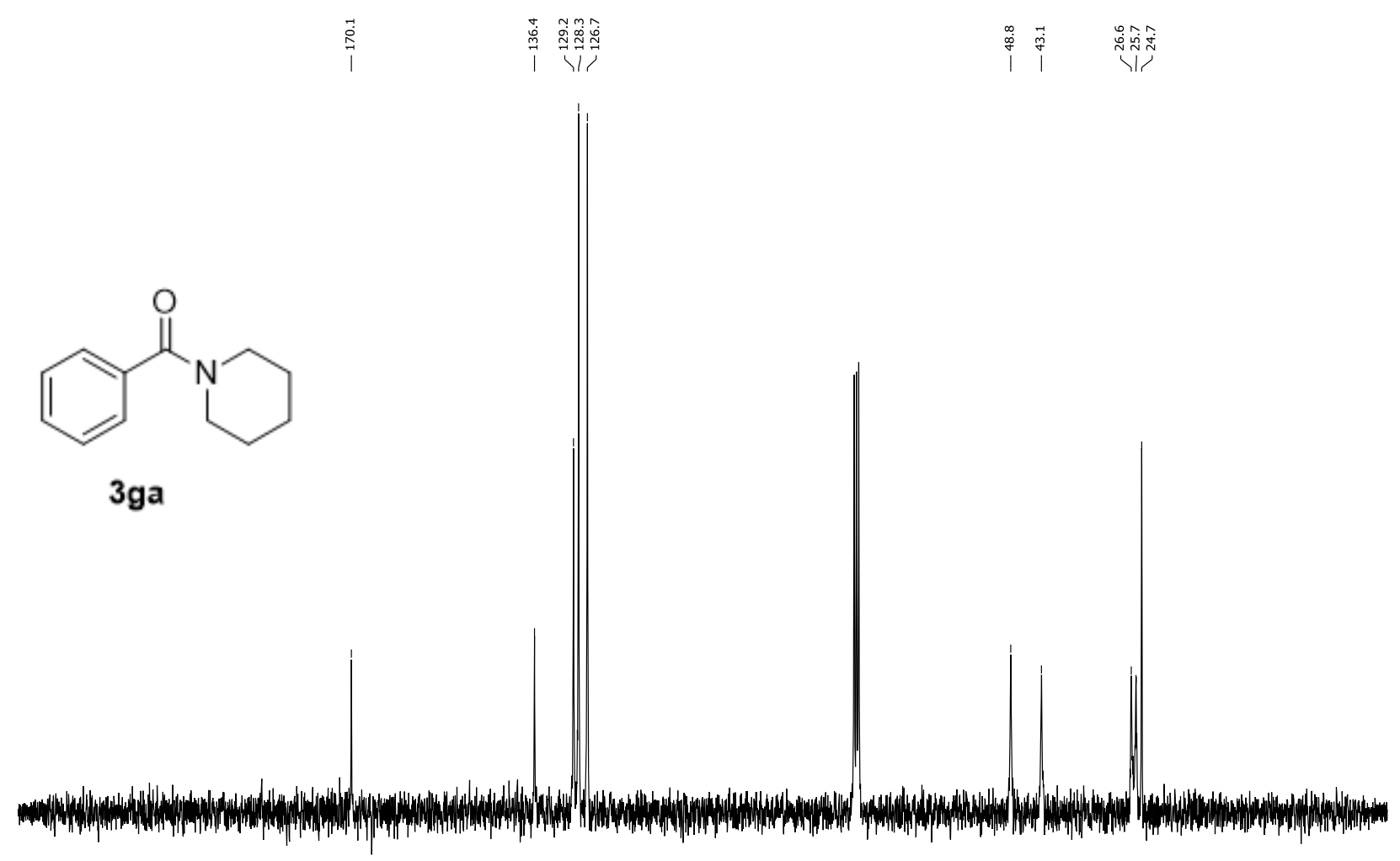

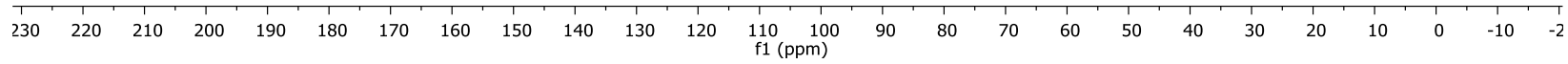

${ }^{13} \mathrm{C}$ NMR (75 MHz, Chloroform- $d$ ) phenyl(piperidin-1-yl)methanone (3ga)
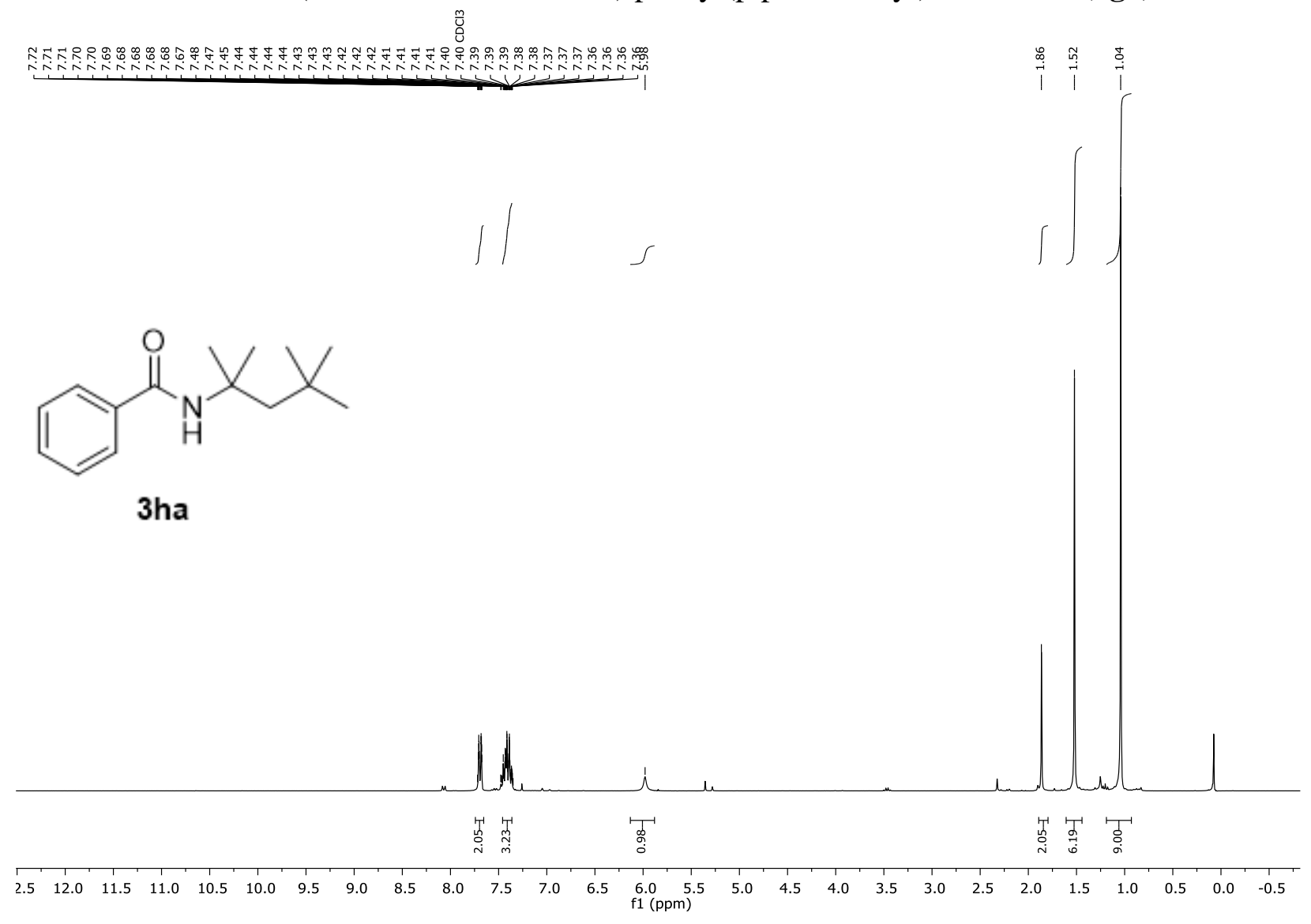

${ }^{1} \mathrm{H}$ NMR (300 MHz, Chloroform- $d$ ) N-(2,4,4-trimethylpentan-2-yl)benzamide (3ha) 


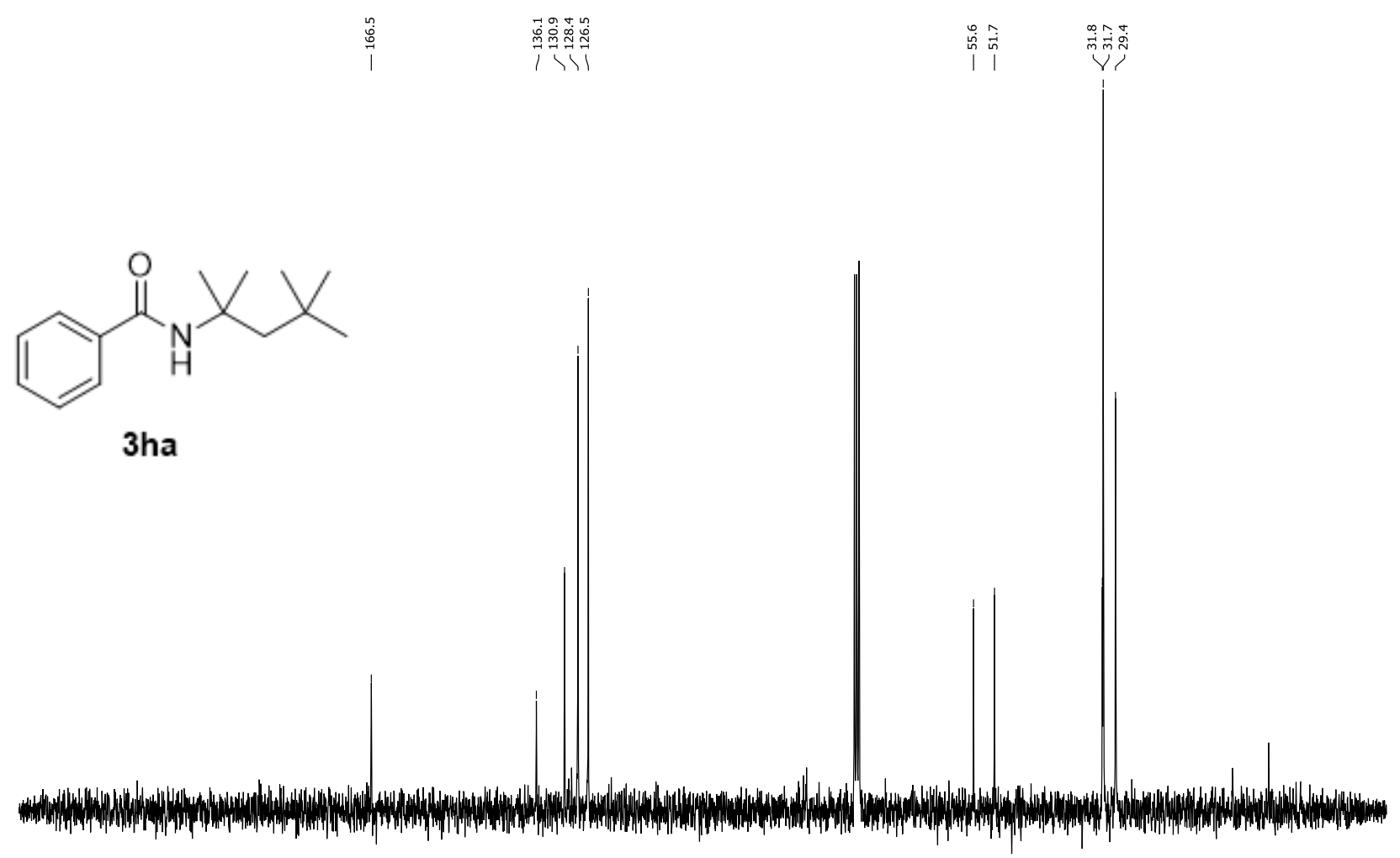

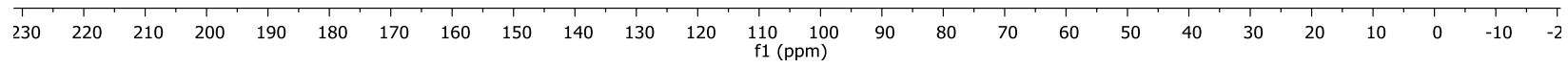

${ }^{13} \mathrm{C}$ NMR (75 MHz, Chloroform- $d$ ) N-(2,4,4-trimethylpentan-2-yl)benzamide (3ha)
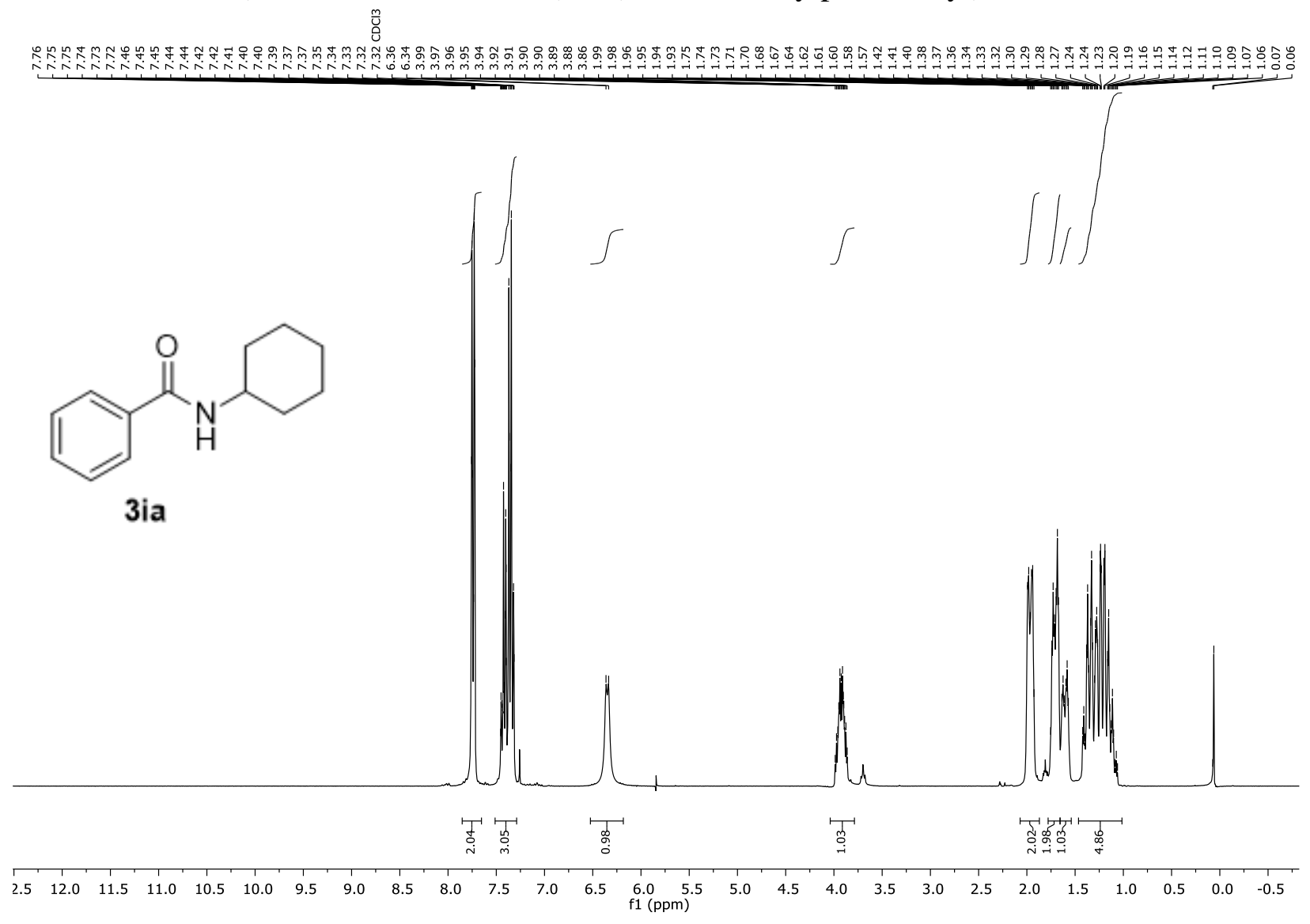

${ }^{1} \mathrm{H}$ NMR (300 MHz, Chloroform- $d$ ) N-cyclohexylbenzamide (3ia) 

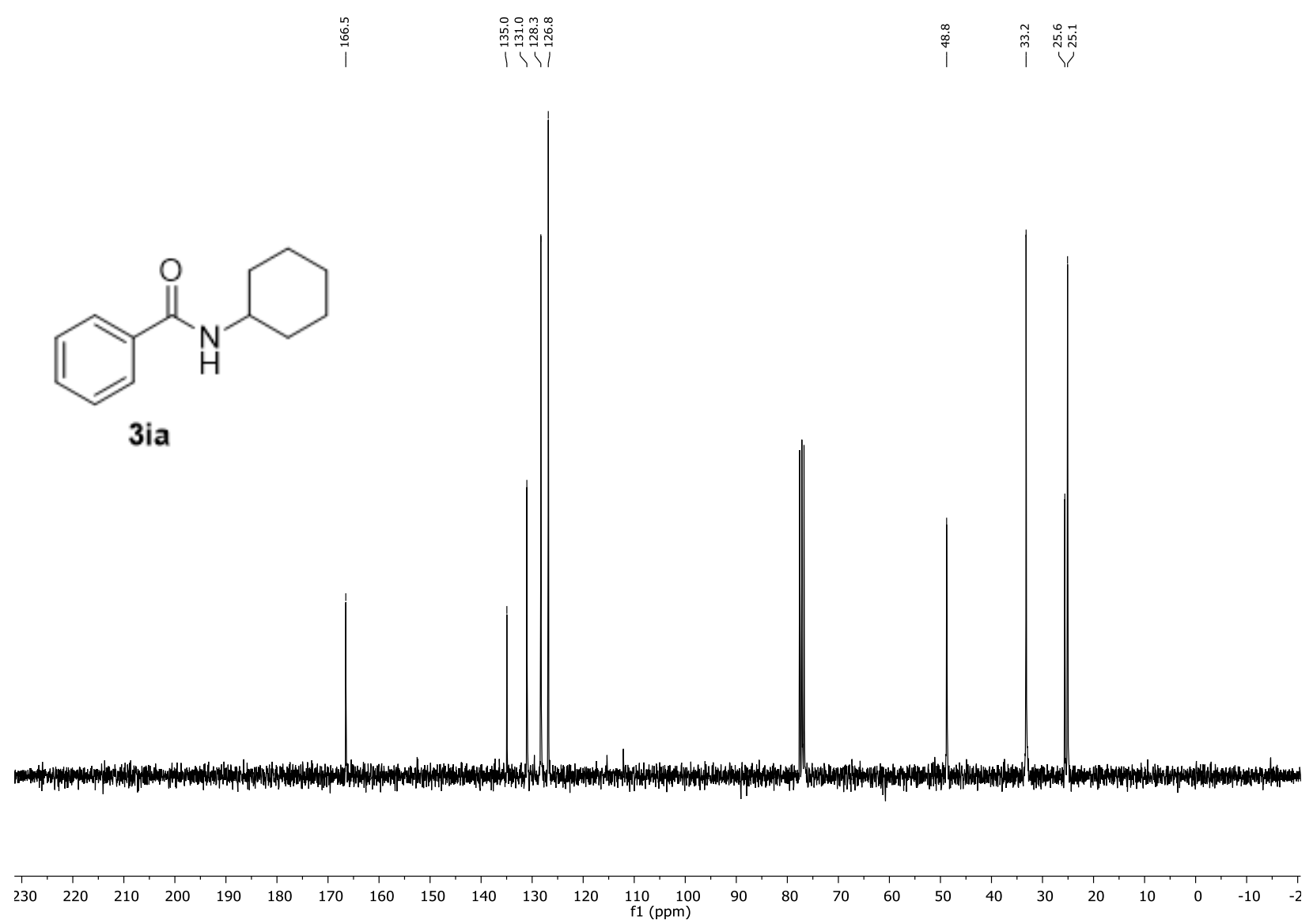

${ }^{13} \mathrm{C}$ NMR (75 MHz, Chloroform-d) N-cyclohexylbenzamide (3ia)

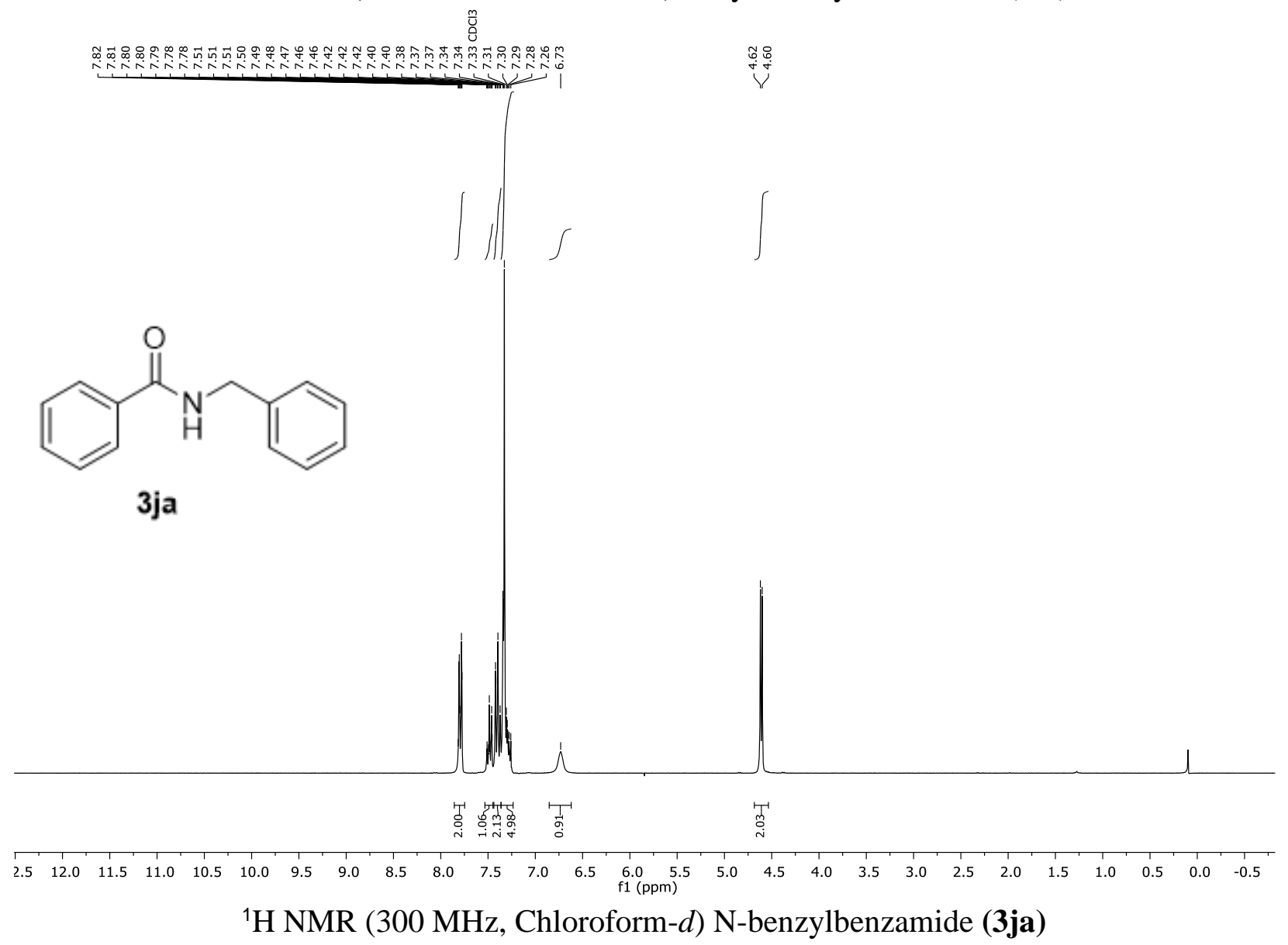



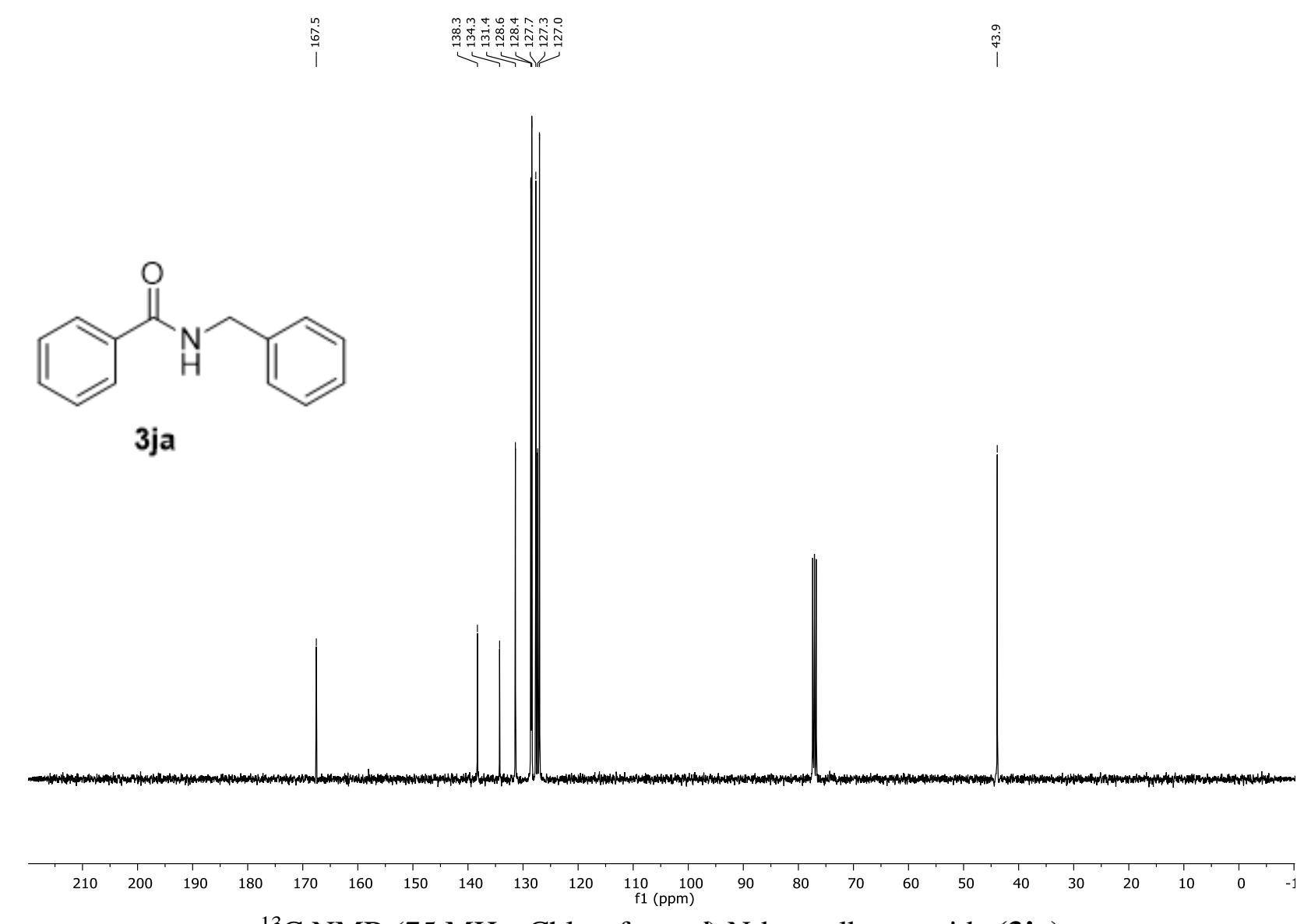

${ }^{13} \mathrm{C}$ NMR (75 MHz, Chloroform- $d$ ) N-benzylbenzamide (3ja)

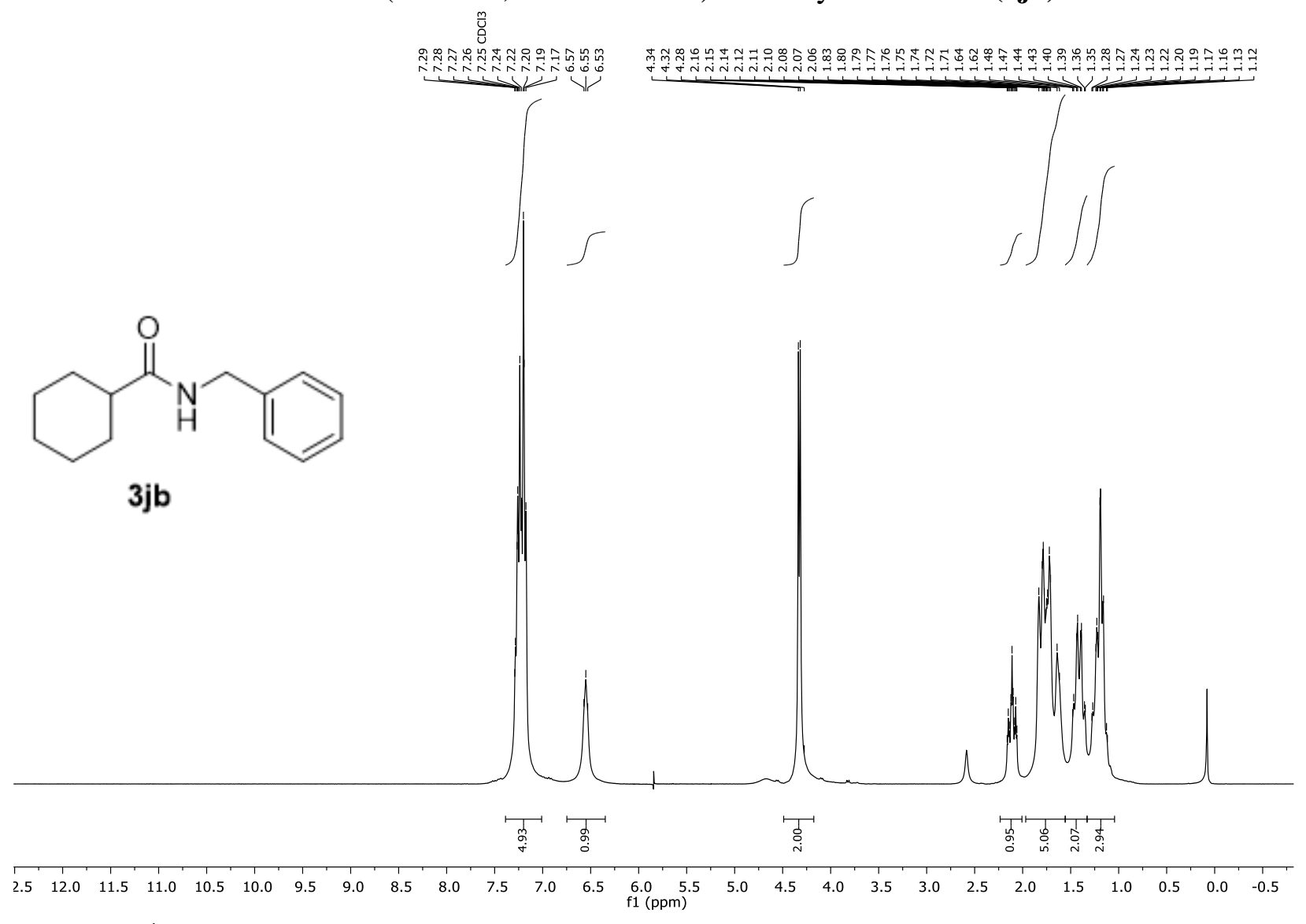

${ }^{1} \mathrm{H}$ NMR (300 MHz, Chloroform- $d$ ) N-benzylcyclohexanecarboxamide (3jb) 

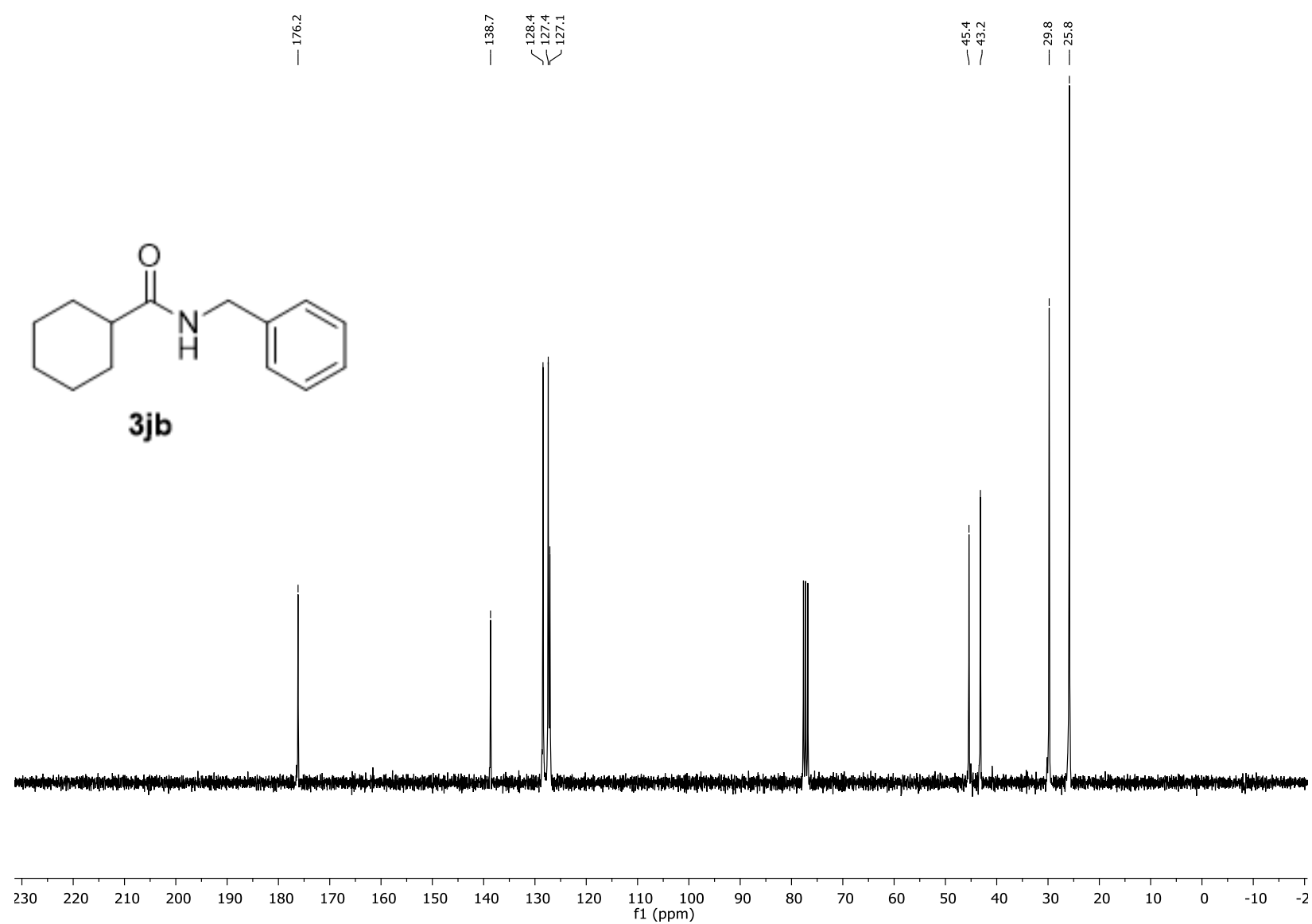

${ }^{13} \mathrm{C}$ NMR (75 MHz, Chloroform- $d$ ) N-benzylcyclohexanecarboxamide (3jb)

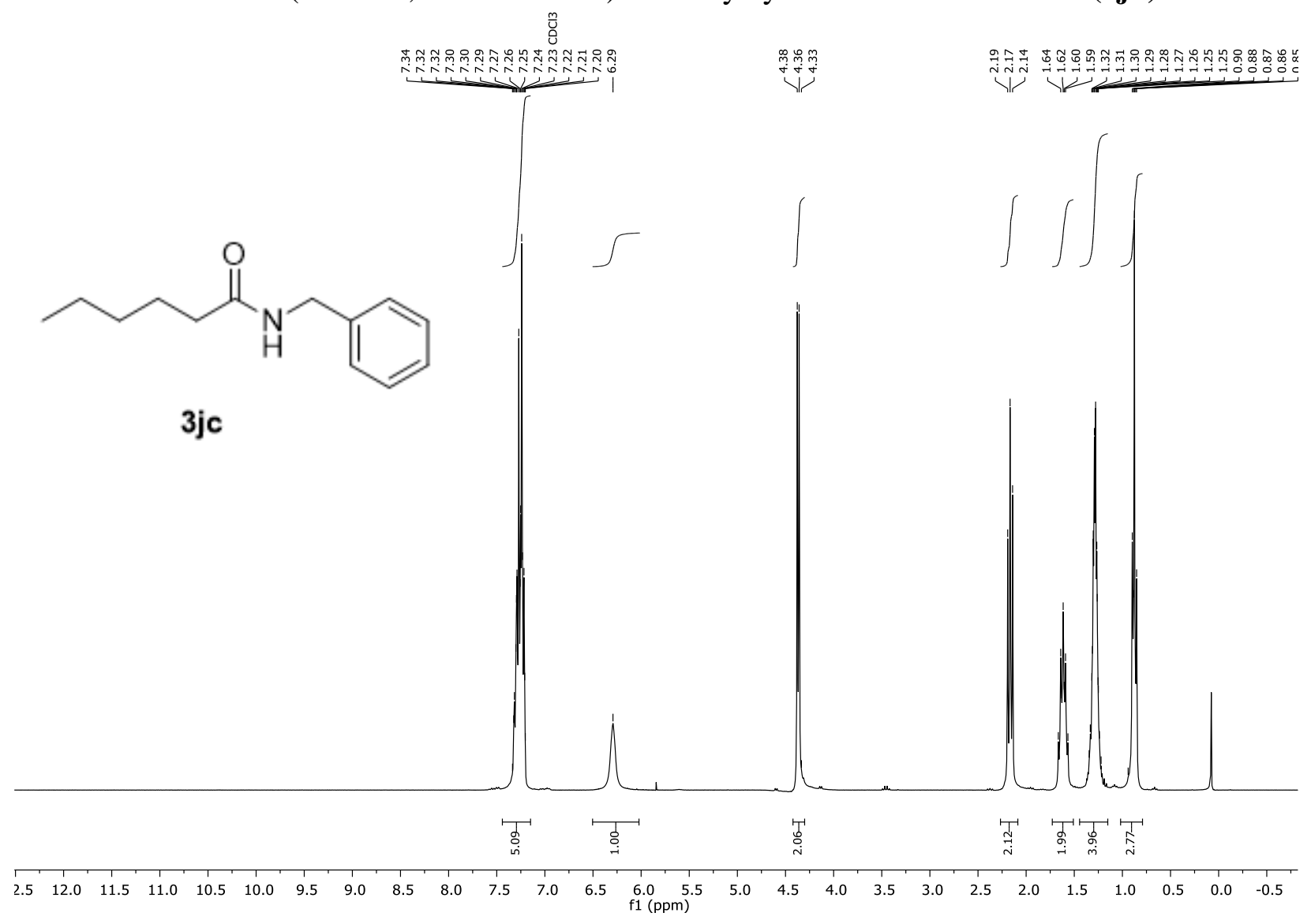

${ }^{1} \mathrm{H}$ NMR (300 MHz, Chloroform- $d$ ) N-benzylhexanamide (3jc) 

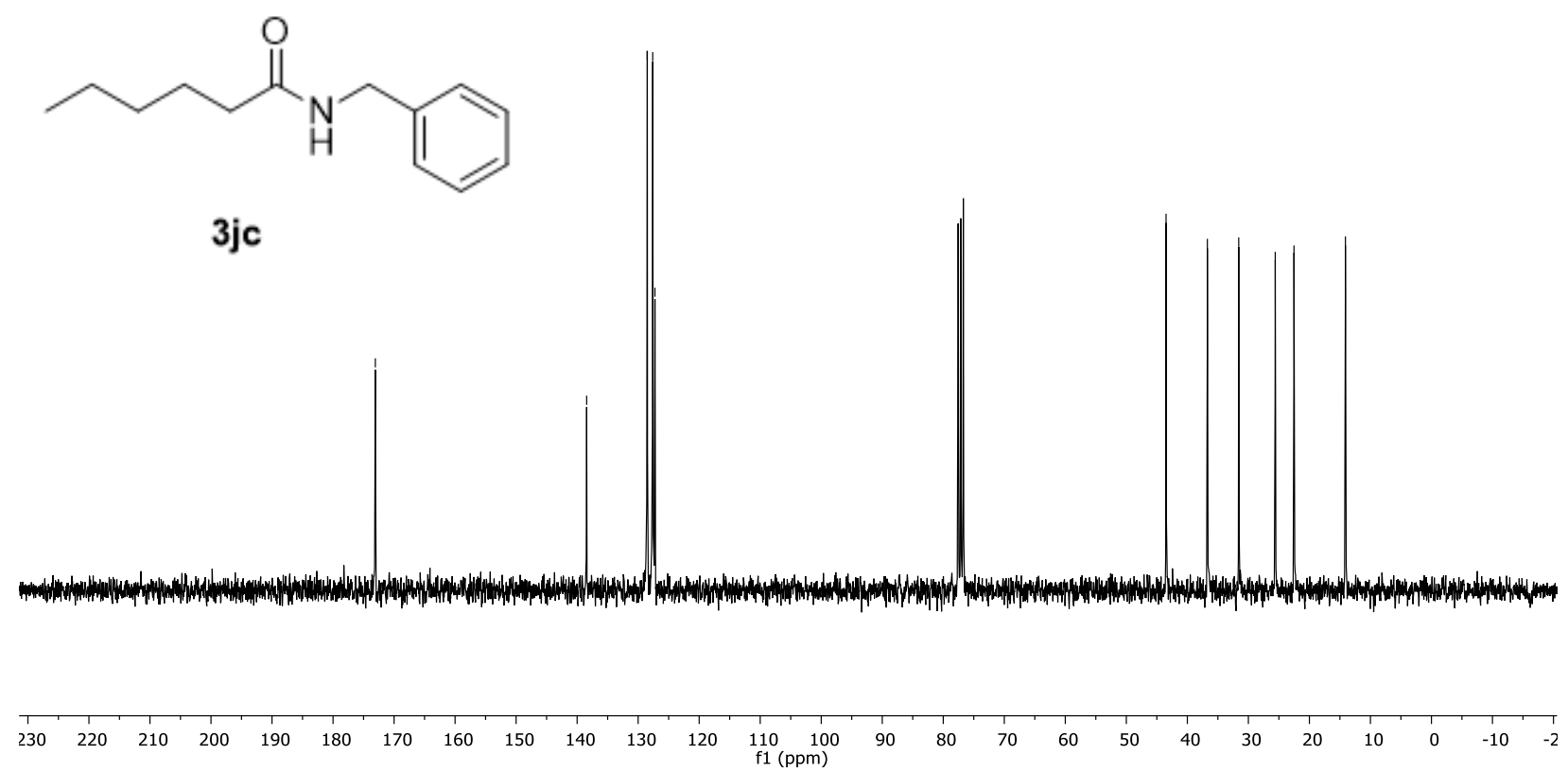

${ }^{13} \mathrm{C}$ NMR (75 MHz, Chloroform- $d$ ) N-benzylhexanamide (3jc)

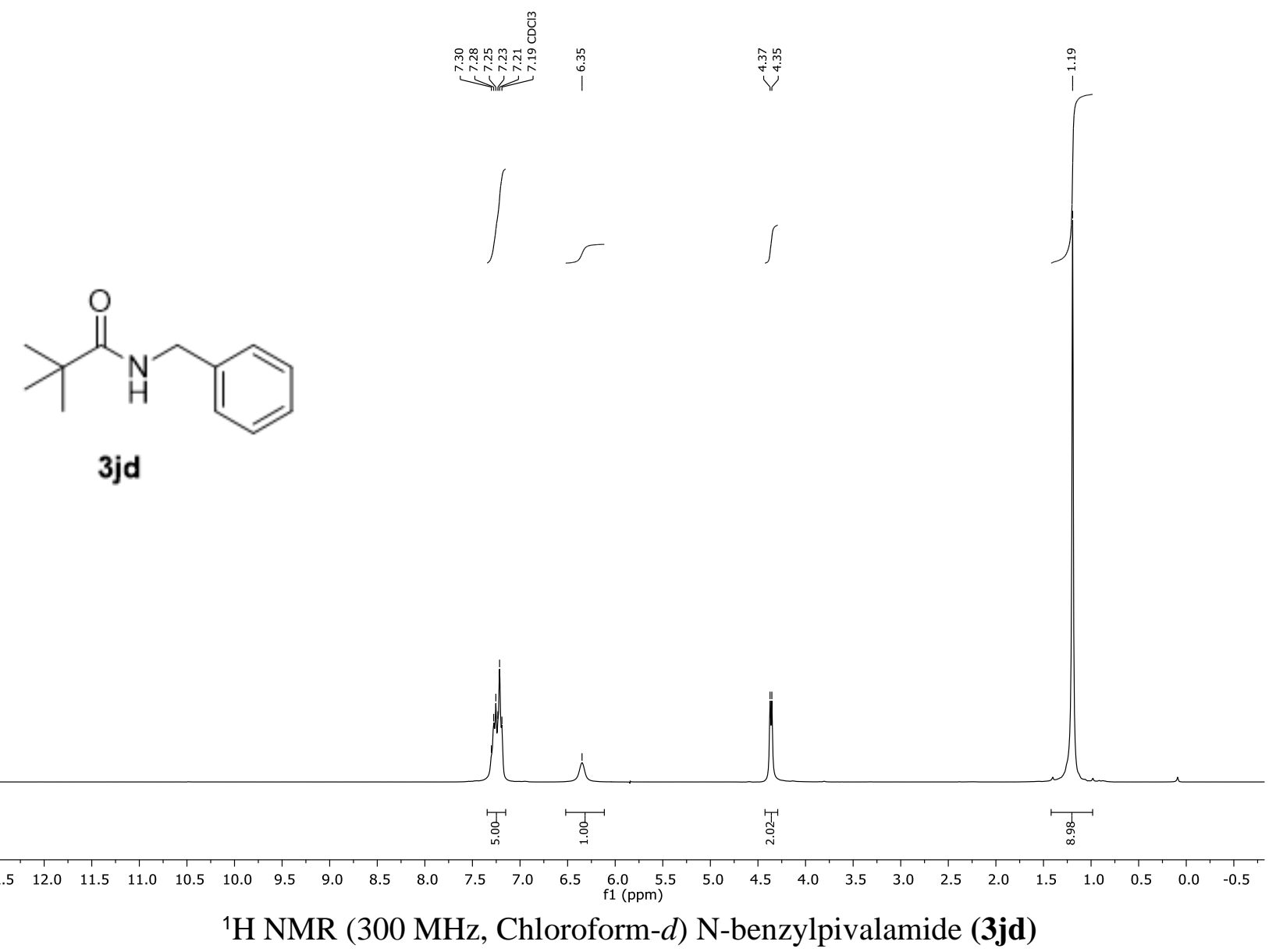



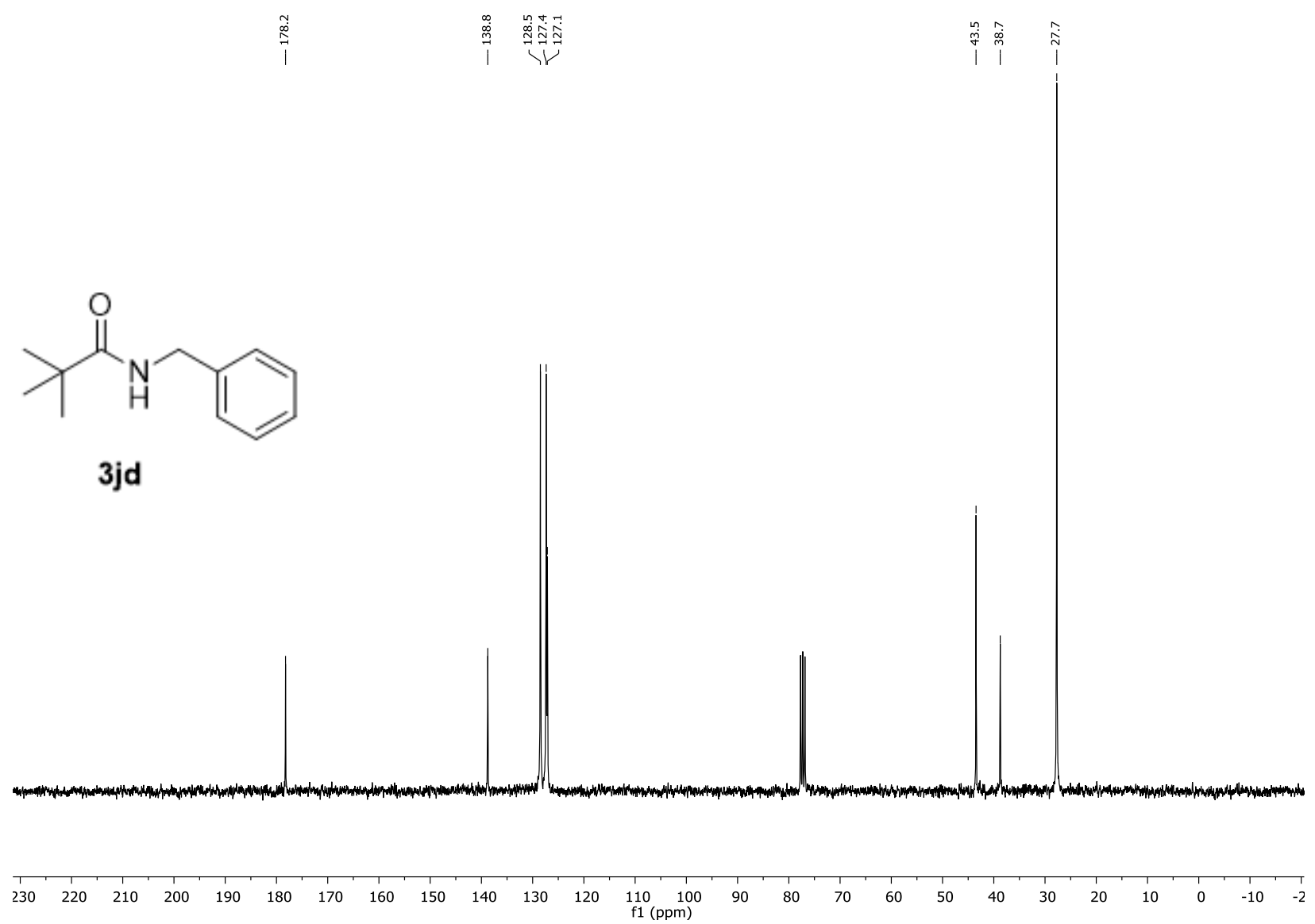

${ }^{13} \mathrm{C}$ NMR (75 MHz, Chloroform- $d$ ) N-benzylpivalamide (3jd)

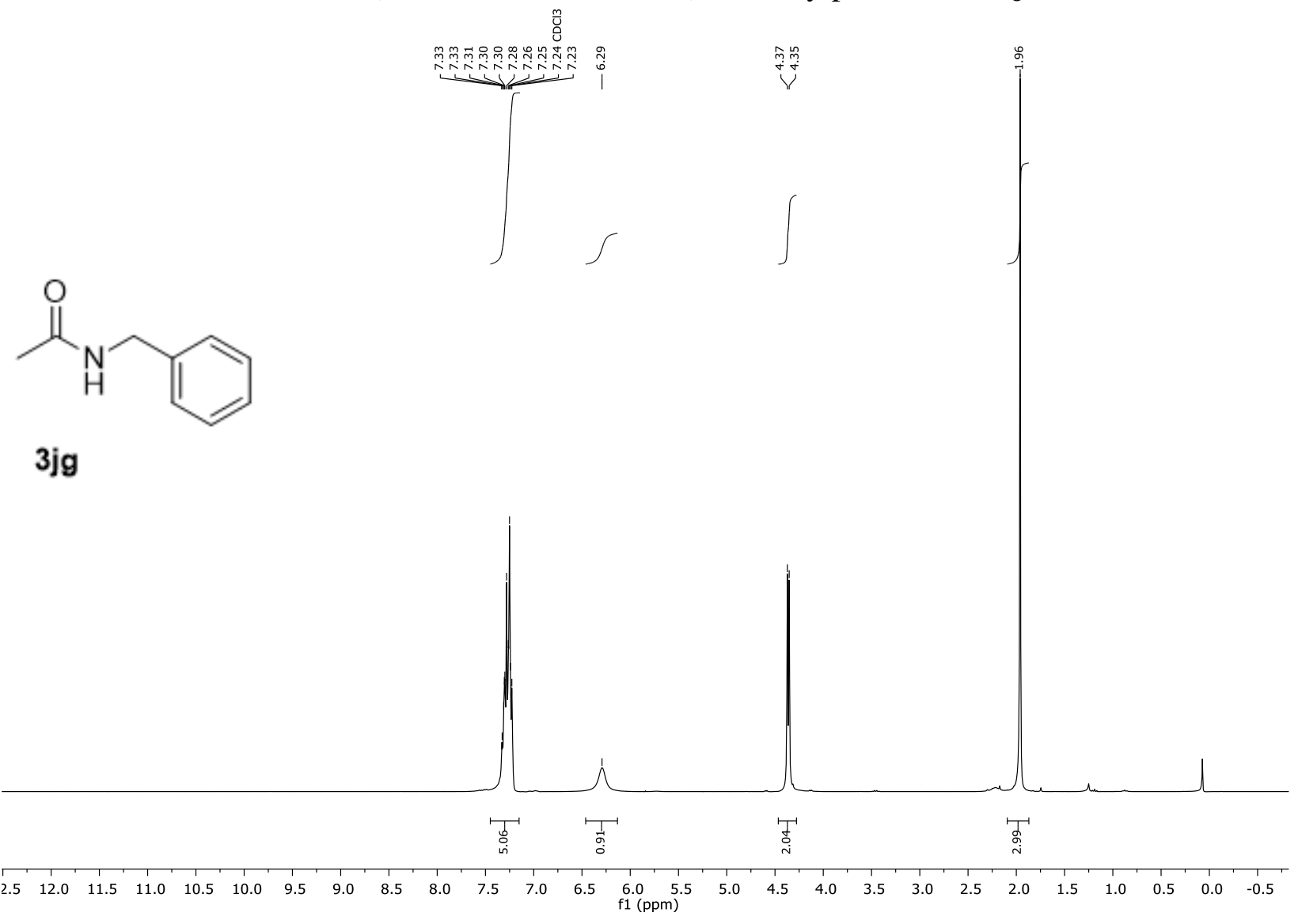

${ }^{1} \mathrm{H}$ NMR (300 MHz, Chloroform- $d$ ) N-benzylacetamide (3jg) 


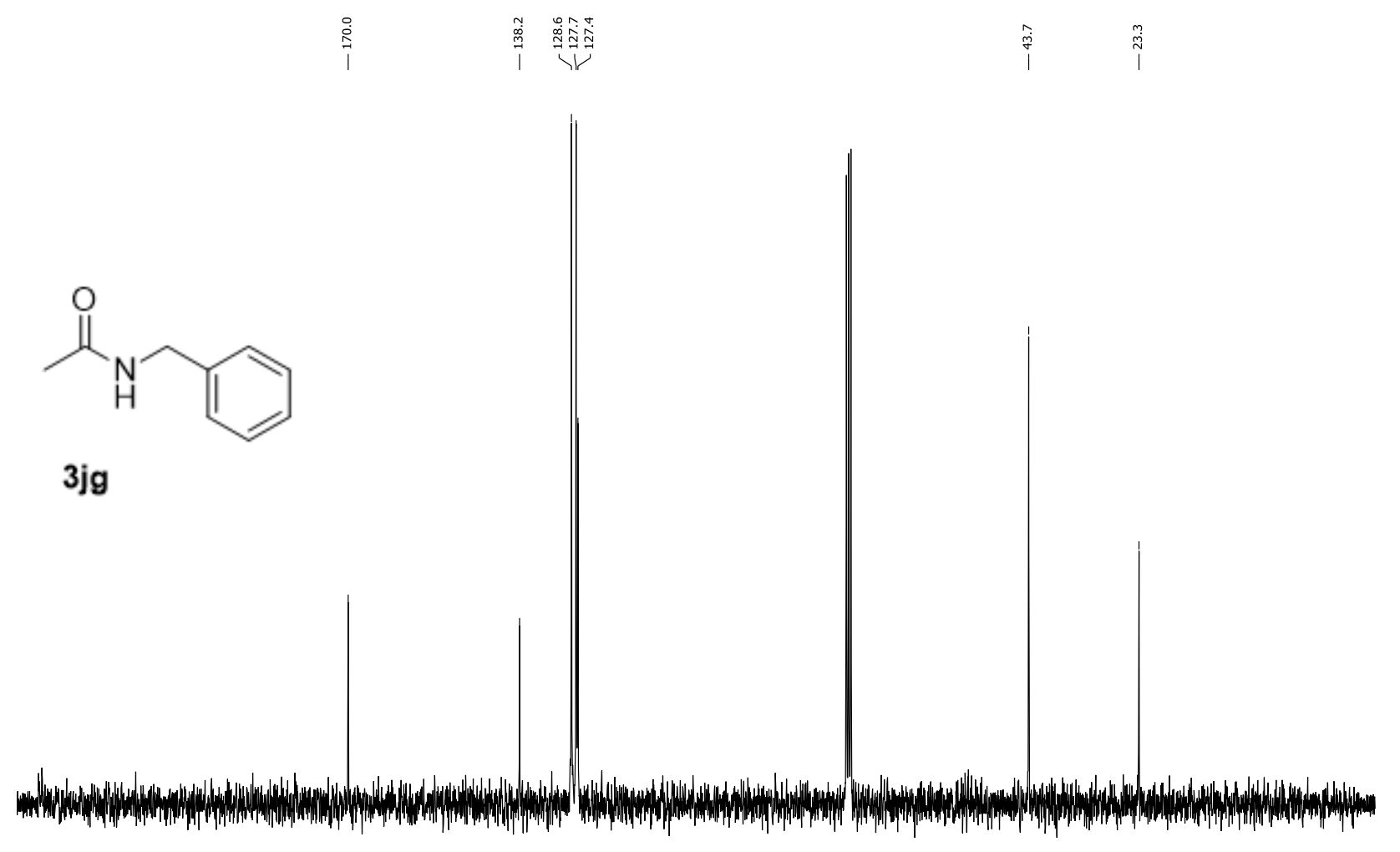

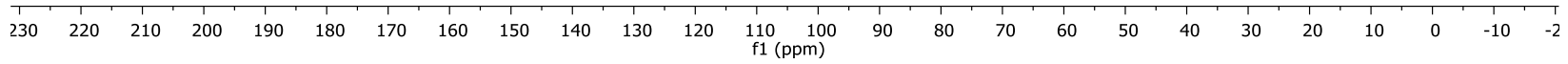

${ }^{13} \mathrm{C}$ NMR (75 MHz, Chloroform-d) N-benzylacetamide (3jg)

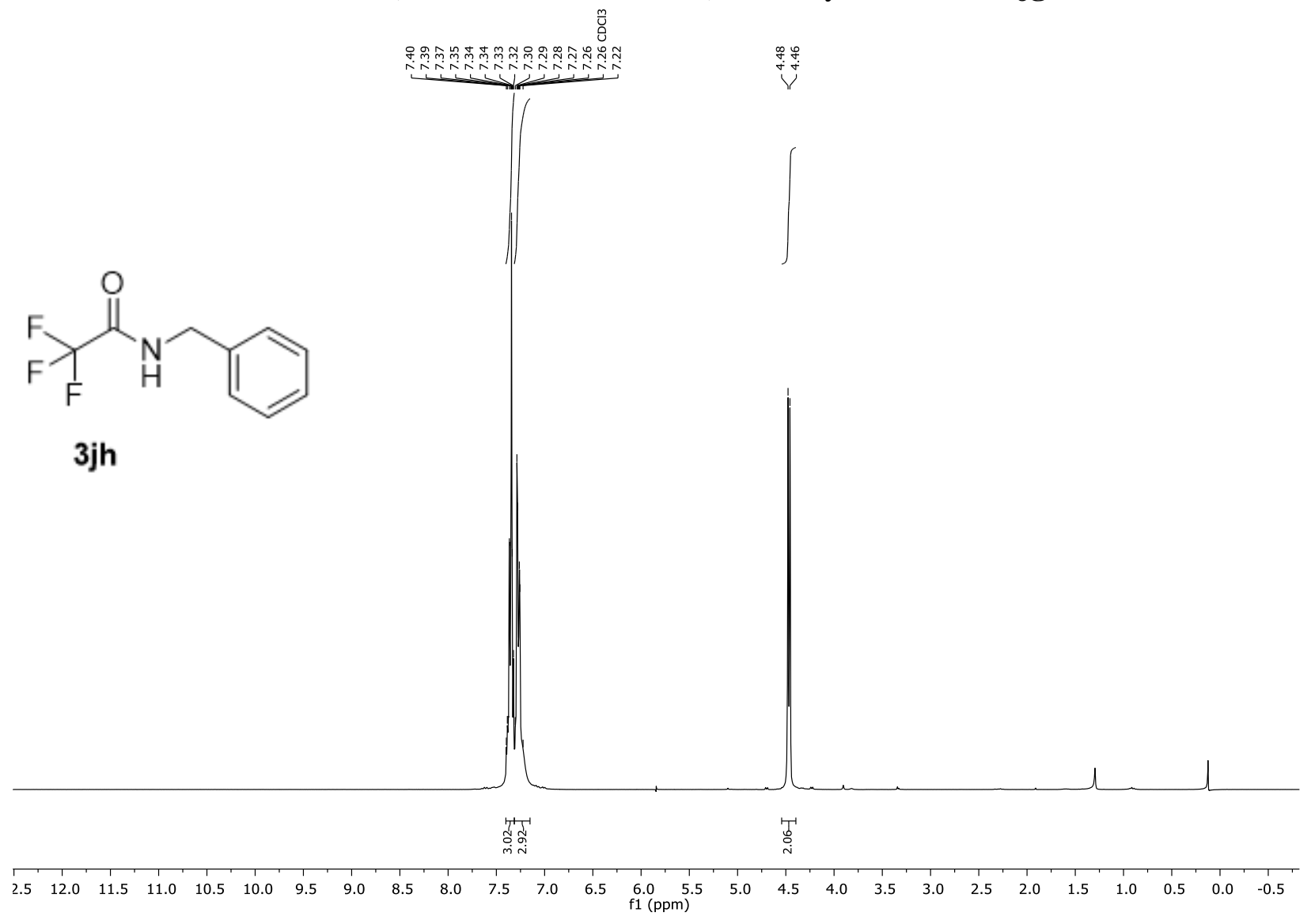

${ }^{1} \mathrm{H}$ NMR (300 MHz, Chloroform- $d$ ) N-benzyl-2,2,2-trifluoroacetamide (3jh) 

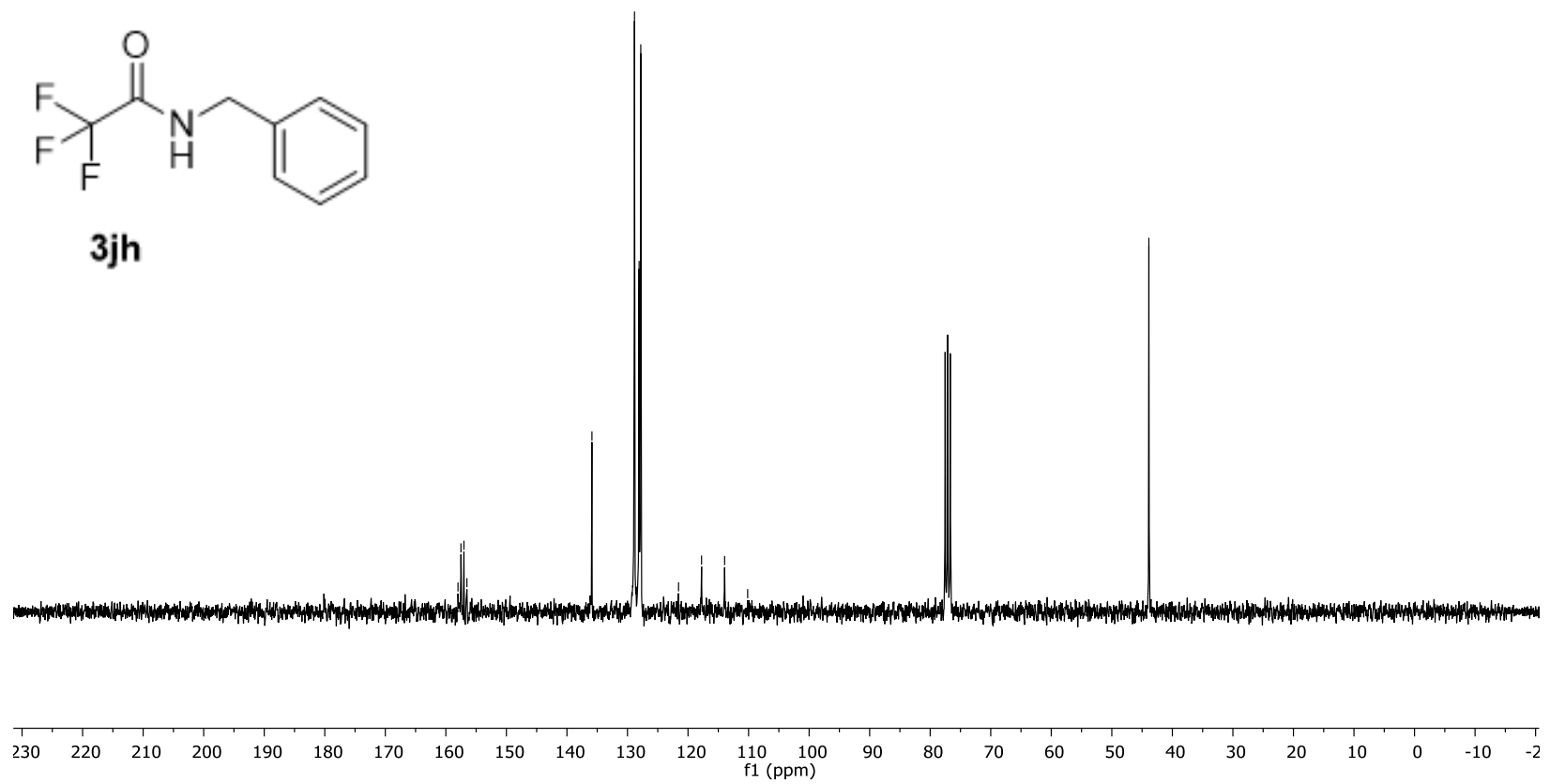

${ }^{13} \mathrm{C}$ NMR (75 MHz, Chloroform- $d$ ) N-benzyl-2,2,2-trifluoroacetamide (3jh)<smiles>O=C(NCc1ccccc1)C(F)(F)F</smiles>

$3 \mathrm{jh}$

${ }^{19} \mathrm{~F}$ NMR (282 MHz, Chloroform- $d$ ) N-benzyl-2,2,2-trifluoroacetamide (3jh) 


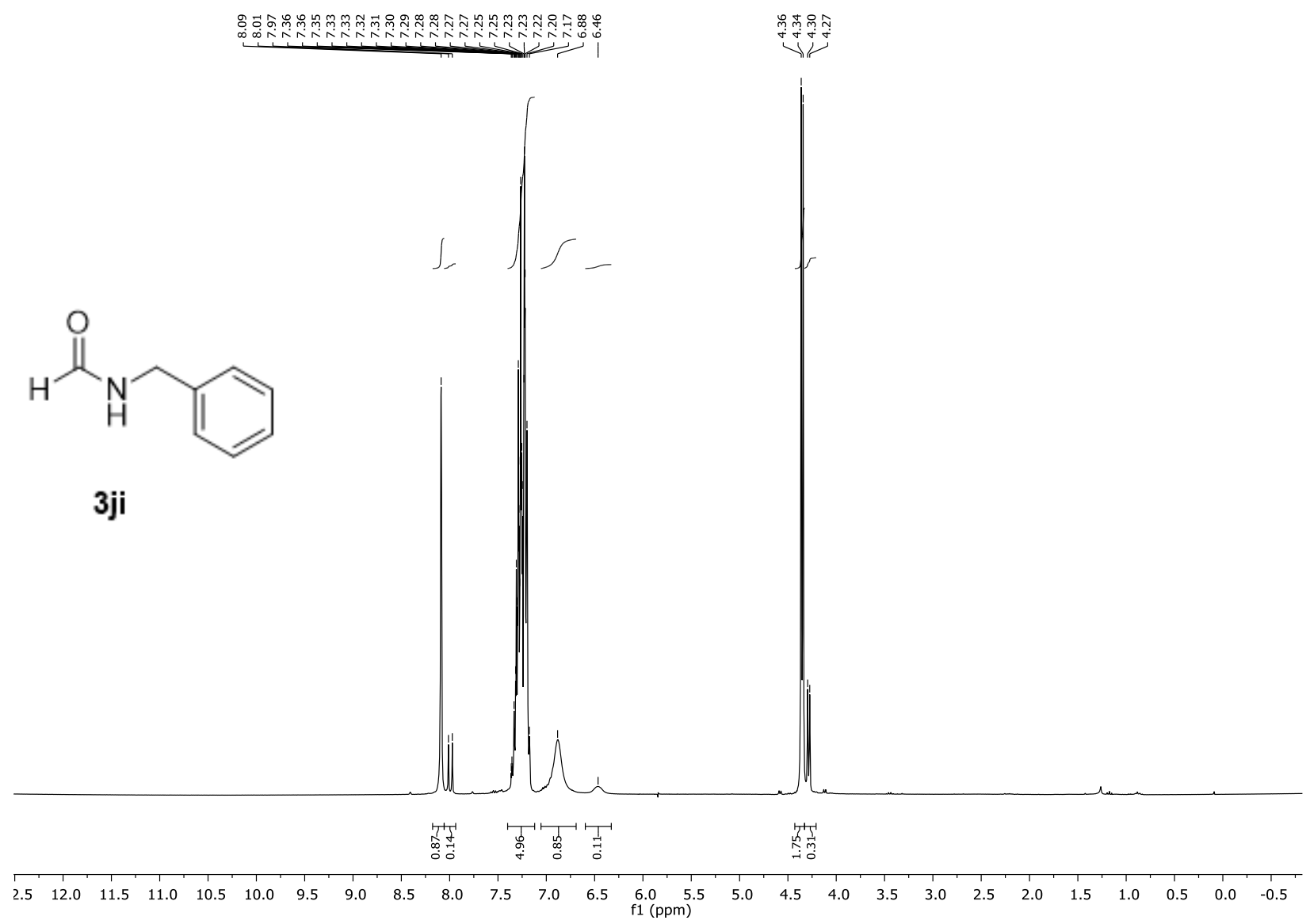

${ }^{1} \mathrm{H}$ NMR (300 MHz, Chloroform- $d$ ) N-benzylformamide (3ji)

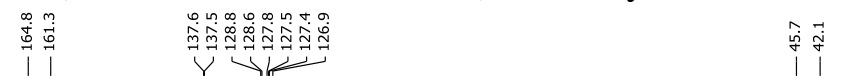<smiles>O=CNCc1ccccc1</smiles>

$3 \mathbf{j i}$

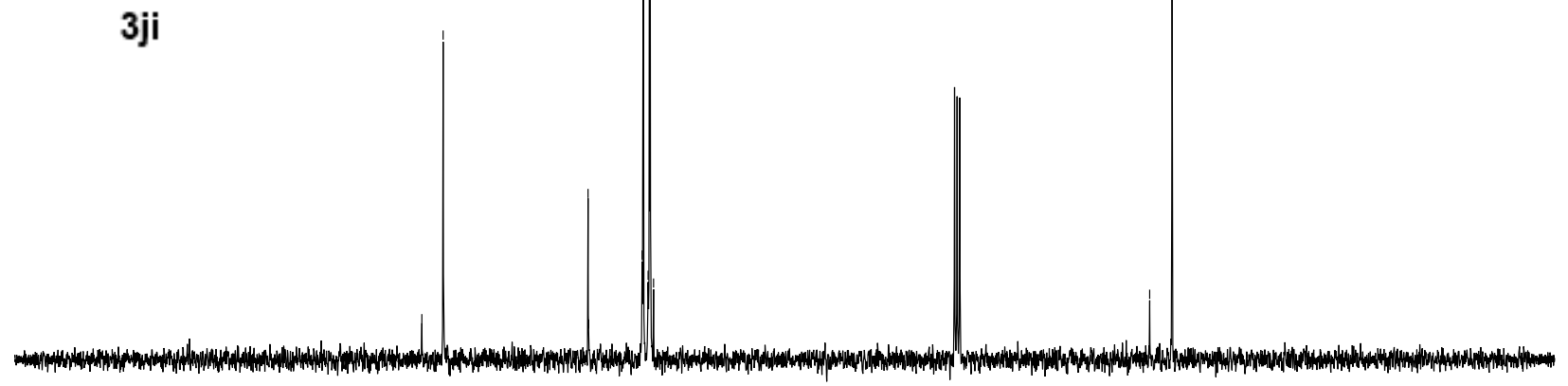

$\begin{array}{llllllllllllllllllllllllllll}1 & 230 & 220 & 210 & 200 & 190 & 180 & 170 & 160 & 150 & 140 & 130 & 120 & \begin{array}{c}110 \\ \mathrm{f} 1(\mathrm{ppm})\end{array} & 100 & 80 & 70 & 60 & 50 & 40 & 30 & 20 & 10 & 0 & -10 & -2\end{array}$

${ }^{13} \mathrm{C}$ NMR (75 MHz, Chloroform- $d$ ) N-benzylformamide (3ji) 


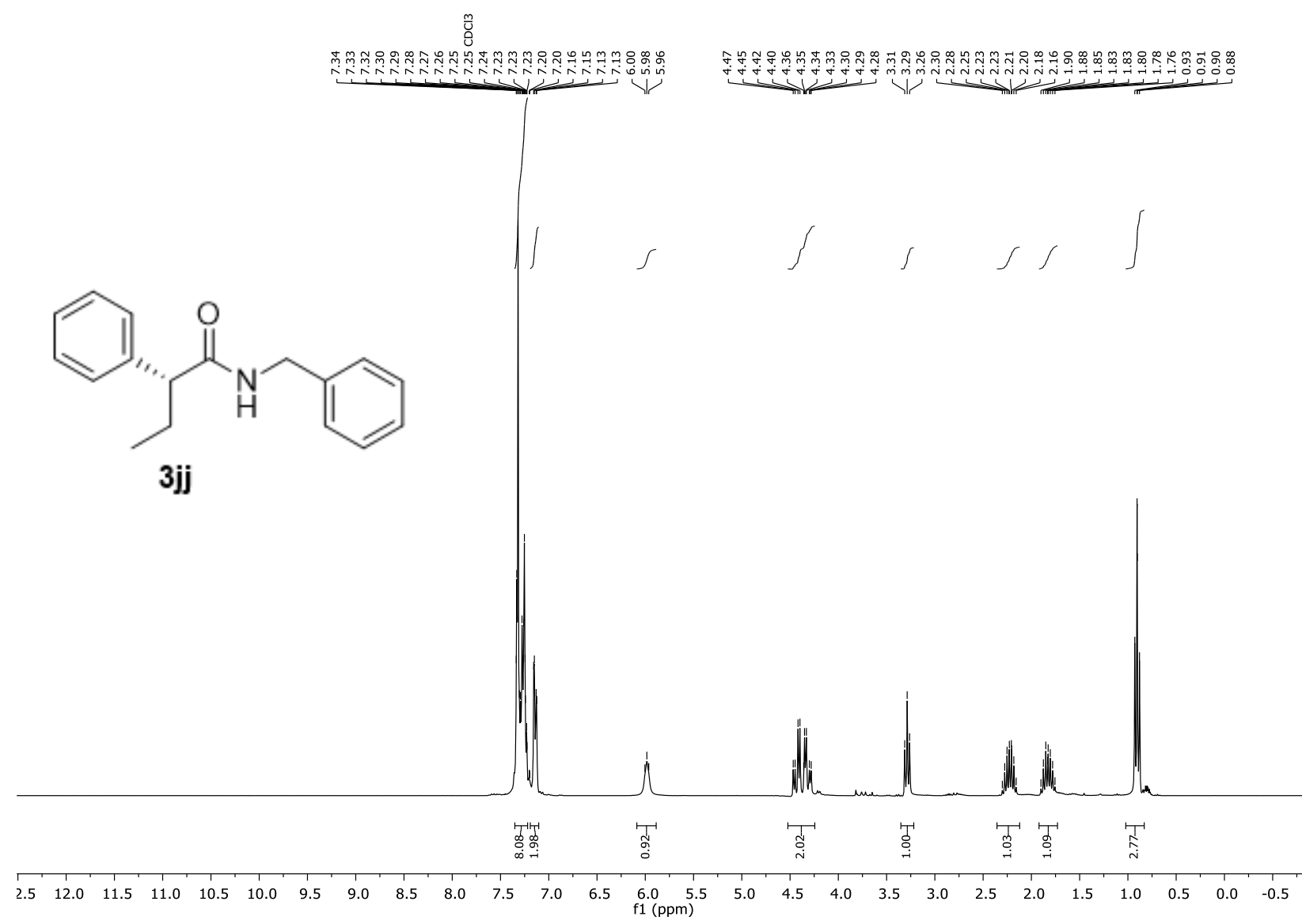

${ }^{1} \mathrm{H}$ NMR (300 MHz, Chloroform- $d$ ) (R)-N-benzyl-2-phenylbutanamide (3jj)

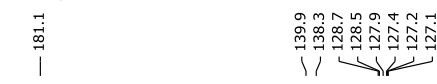

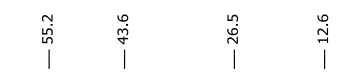<smiles>CC[C@H](C(=O)NCc1ccccc1)c1ccccc1</smiles>

$3 \mathrm{jj}$

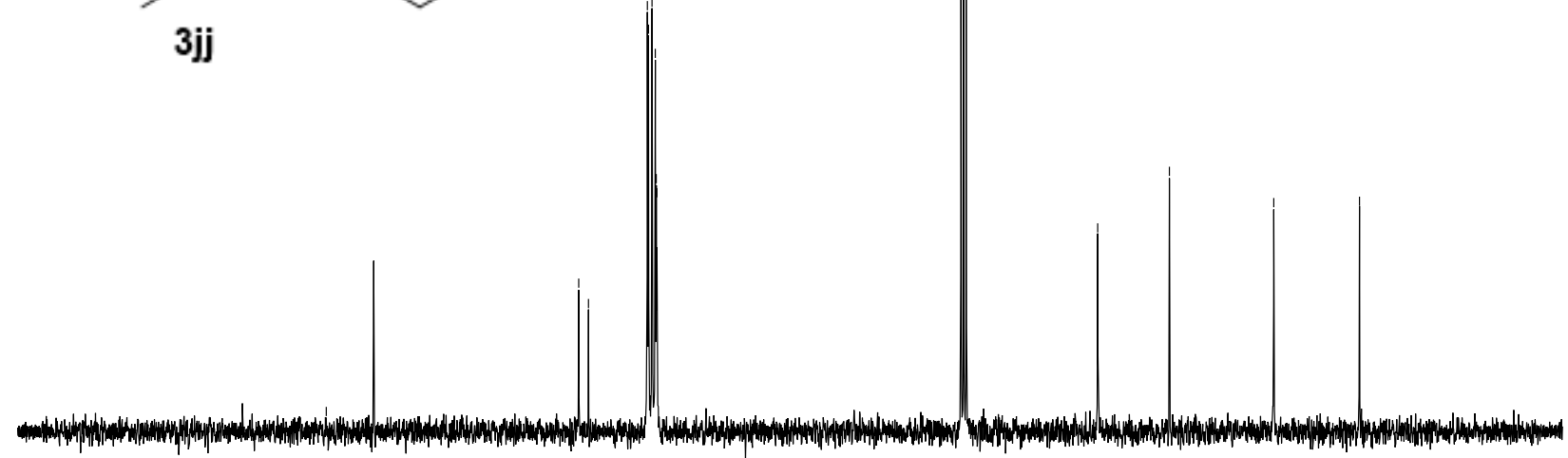

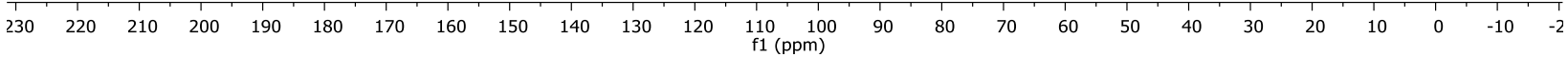

${ }^{13} \mathrm{C}$ NMR (75 MHz, Chloroform- $d$ ) (R)-N-benzyl-2-phenylbutanamide (3jj) 

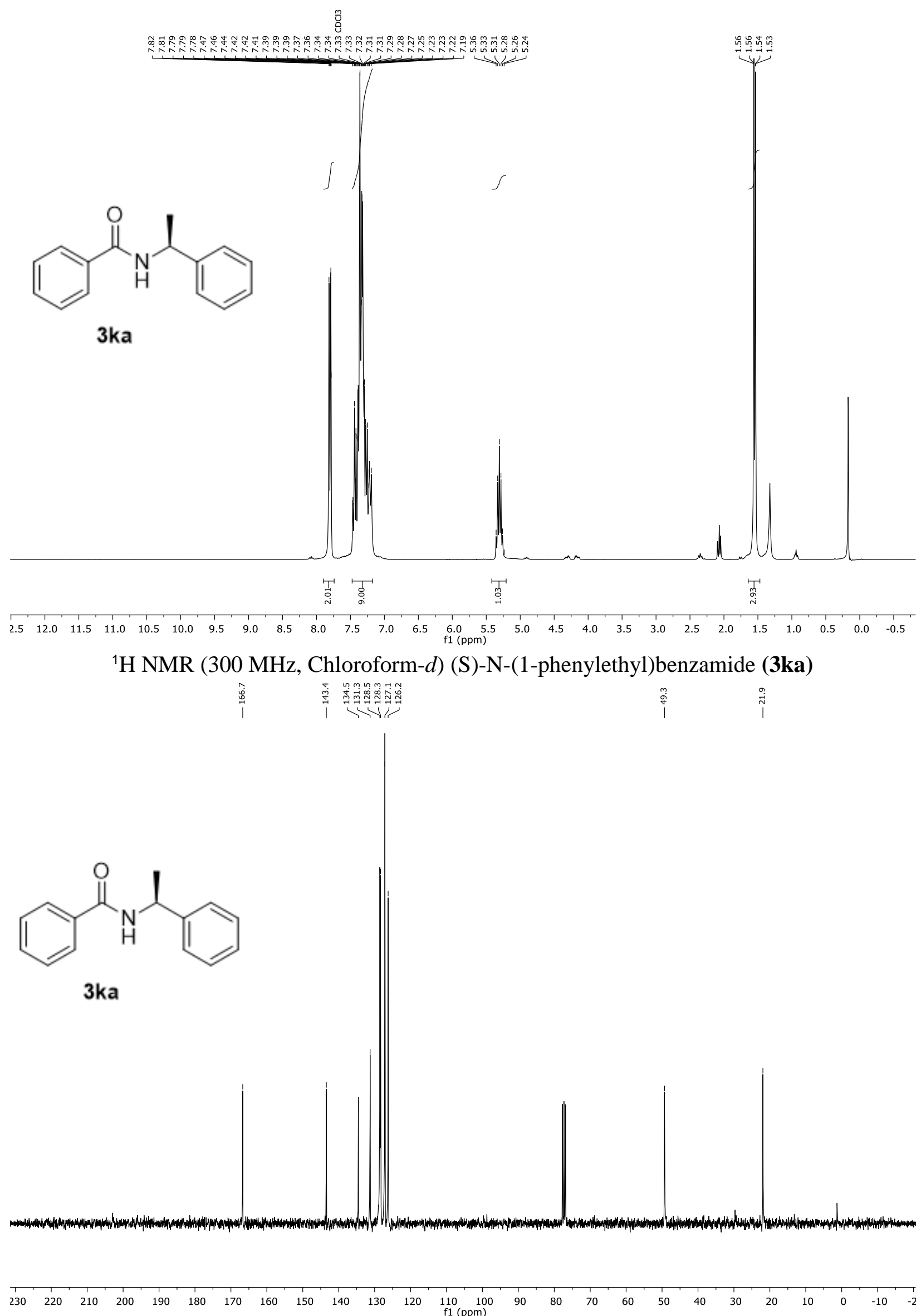

${ }^{13} \mathrm{C}$ NMR (75 MHz, Chloroform- $d$ ) (S)-N-(1-phenylethyl)benzamide (3ka) 

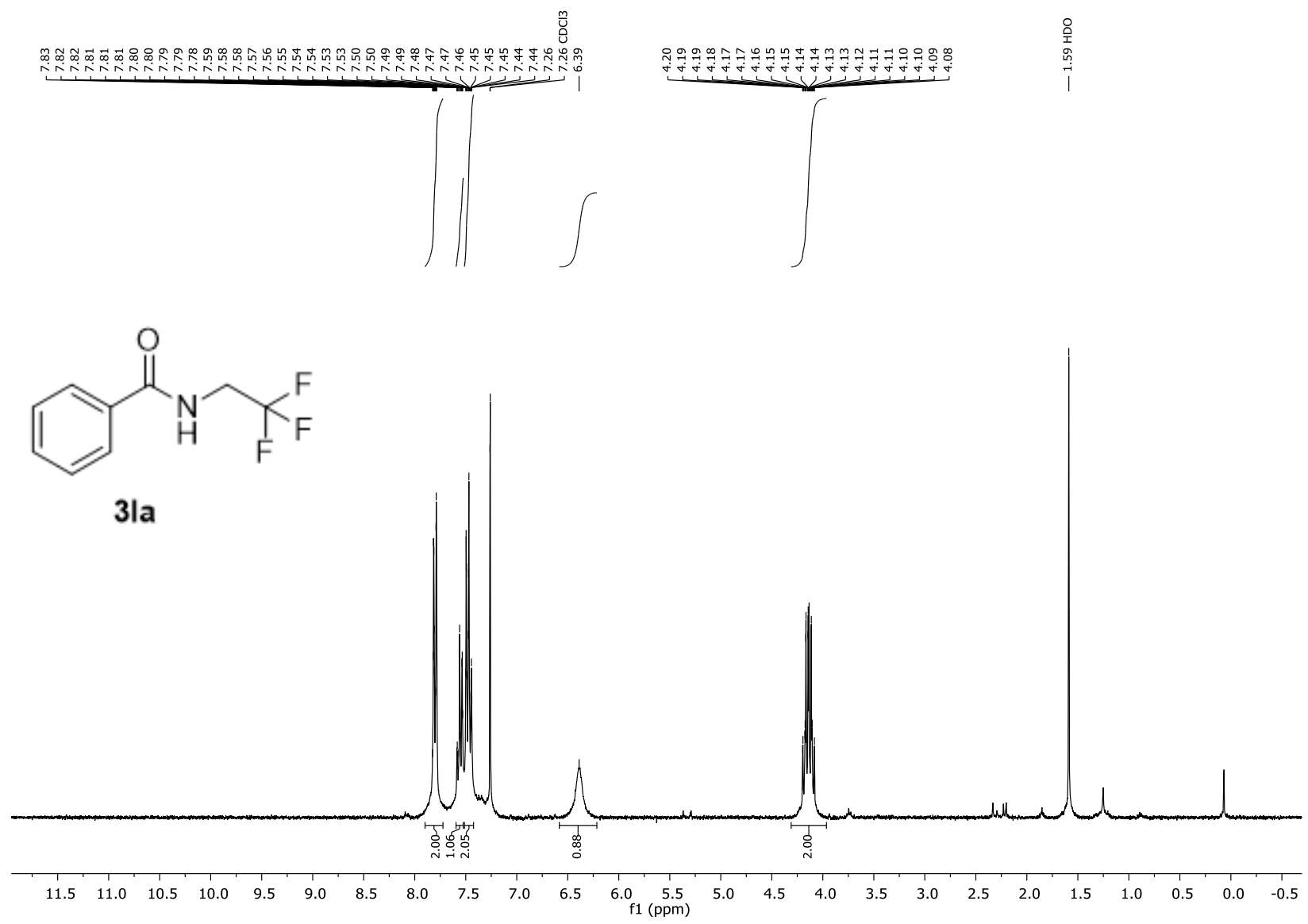

${ }^{1} \mathrm{H}$ NMR (300 MHz, Chloroform- $d$ ) N-(2,2,2-trifluoroethyl)benzamide (3la)
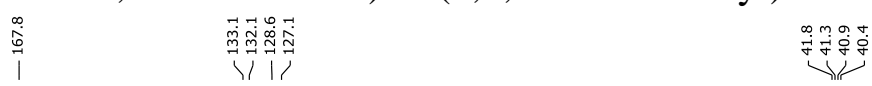<smiles>O=C(NCC(F)(F)F)c1ccccc1</smiles>

3la
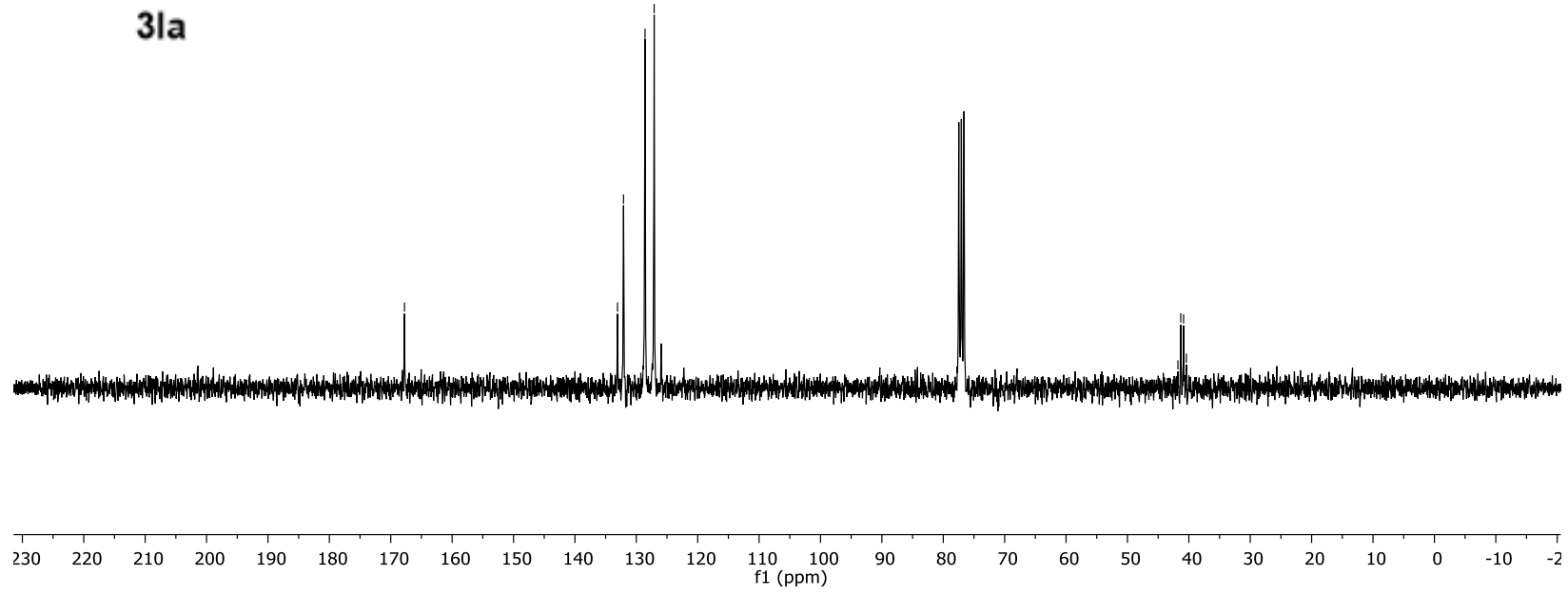

${ }^{13} \mathrm{C}$ NMR (75 MHz, Chloroform- $d$ ) N-(2,2,2-trifluoroethyl)benzamide (3la) 


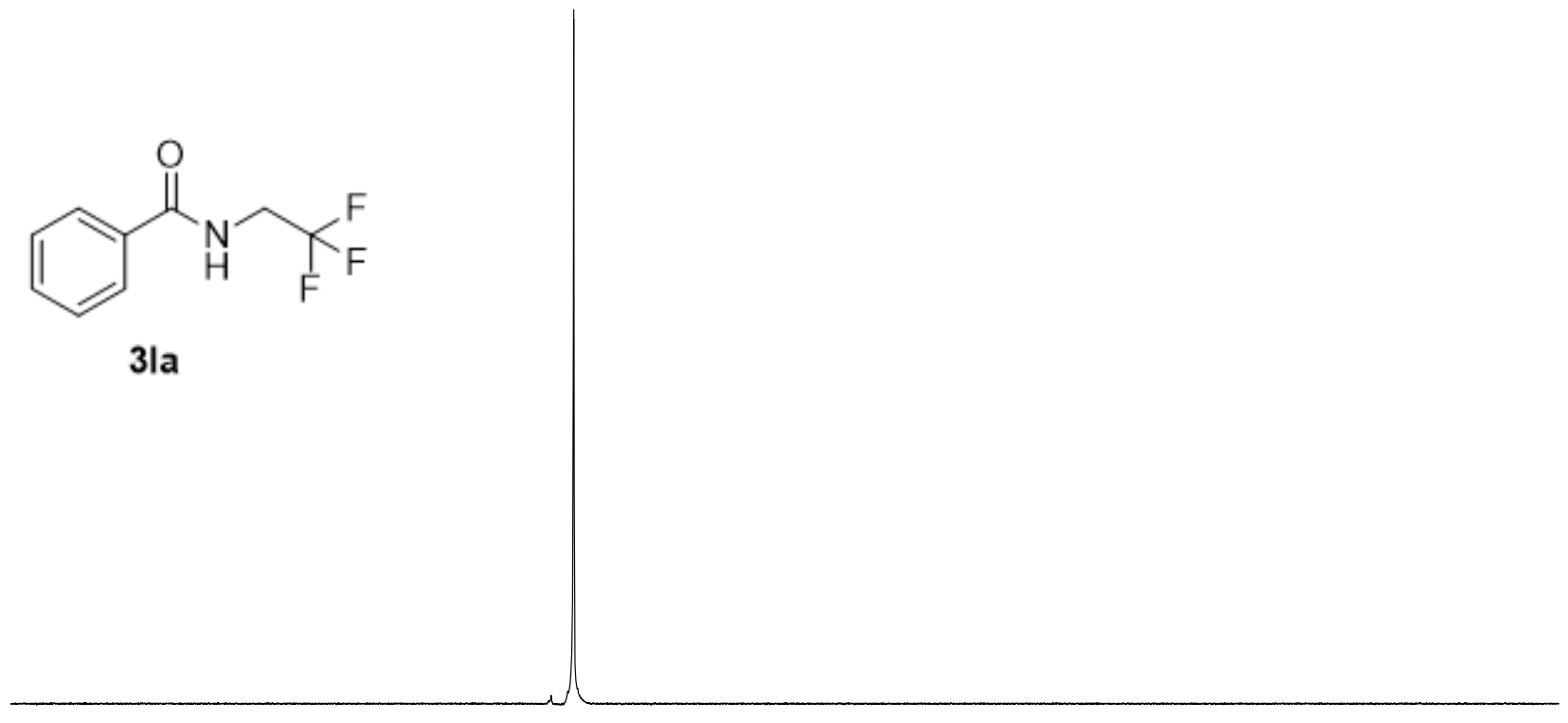

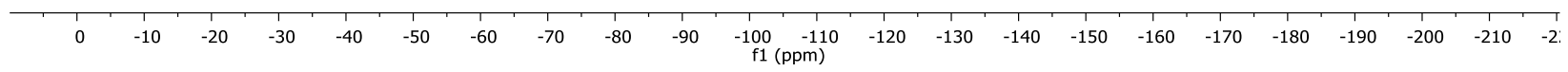

${ }^{19}$ F NMR (282 MHz, Chloroform- $d$ ) N-(2,2,2-trifluoroethyl)benzamide (3la)
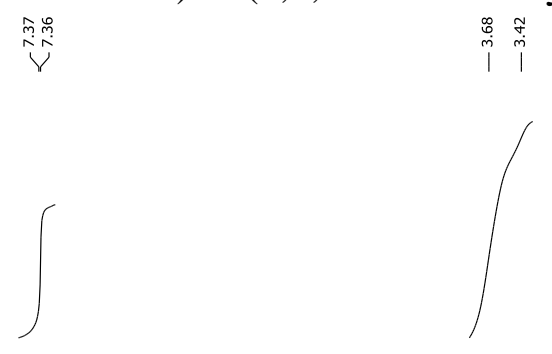<smiles>O=C(c1ccccc1)N1CCOCC1</smiles>

$3 \mathrm{ma}$

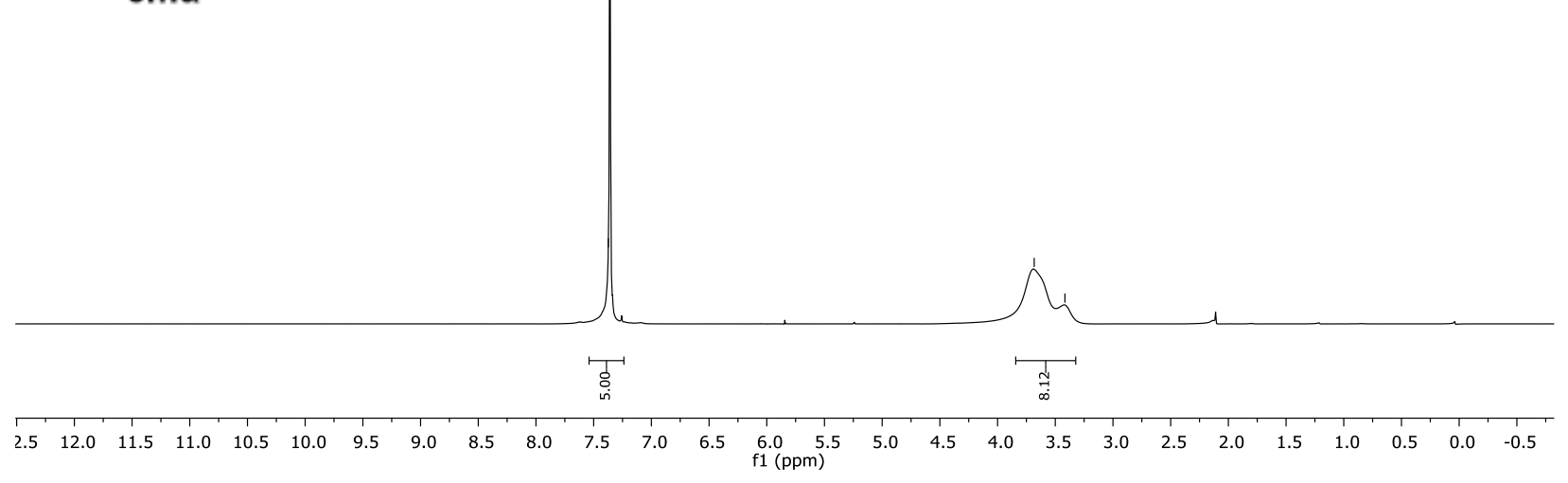

${ }^{1} \mathrm{H}$ NMR (300 MHz, Chloroform- $d$ ) morpholino(phenyl)methanone (3ma) 


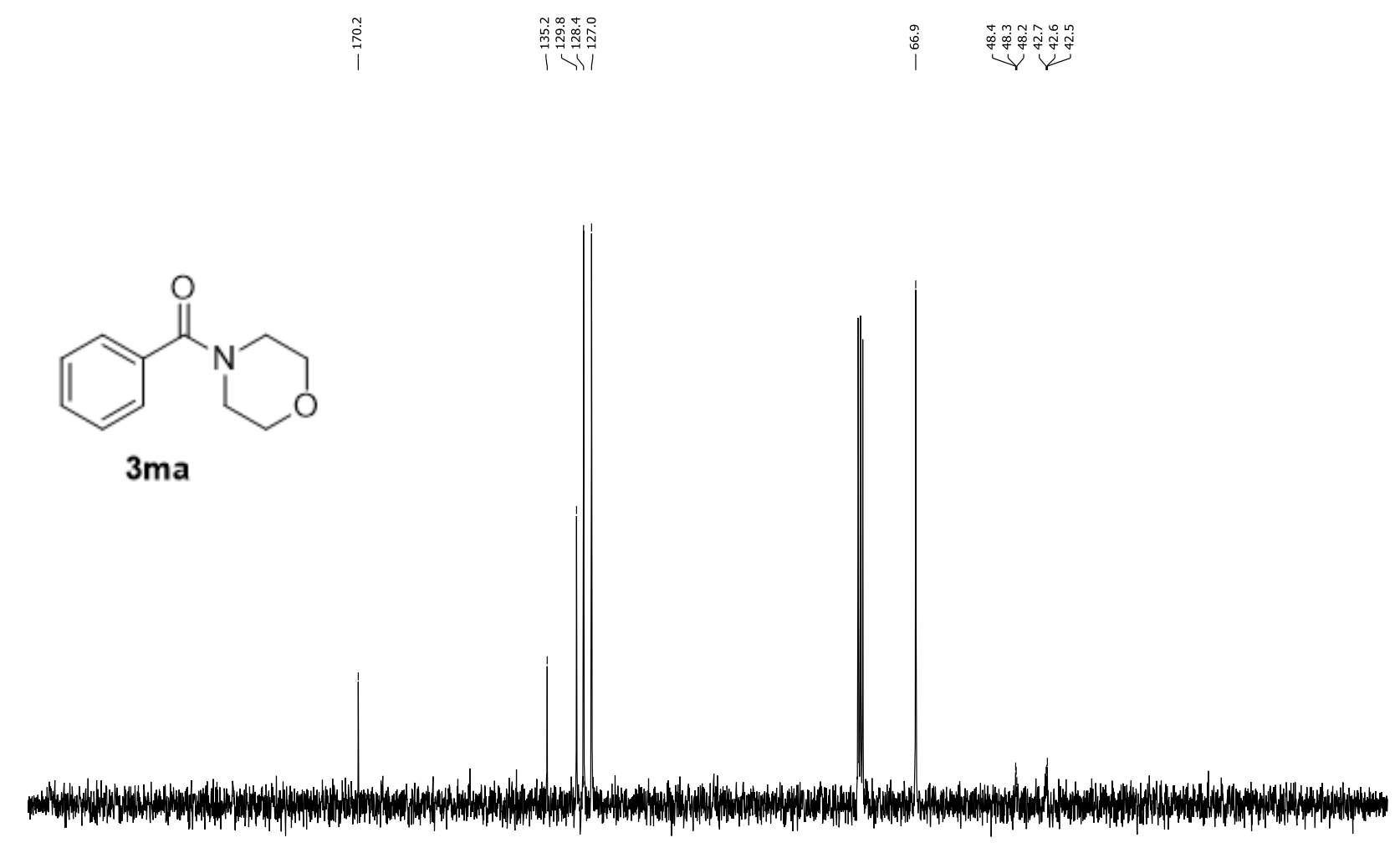

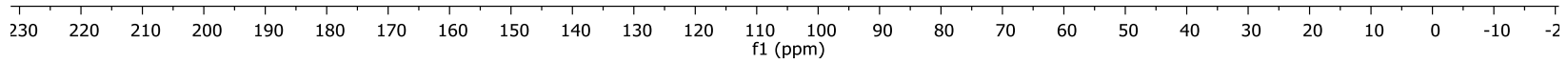

${ }^{13} \mathrm{C}$ NMR (75 MHz, Chloroform- $d$ ) morpholino(phenyl)methanone (3ma)

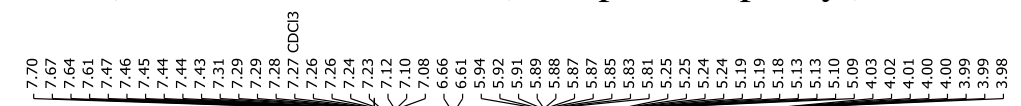

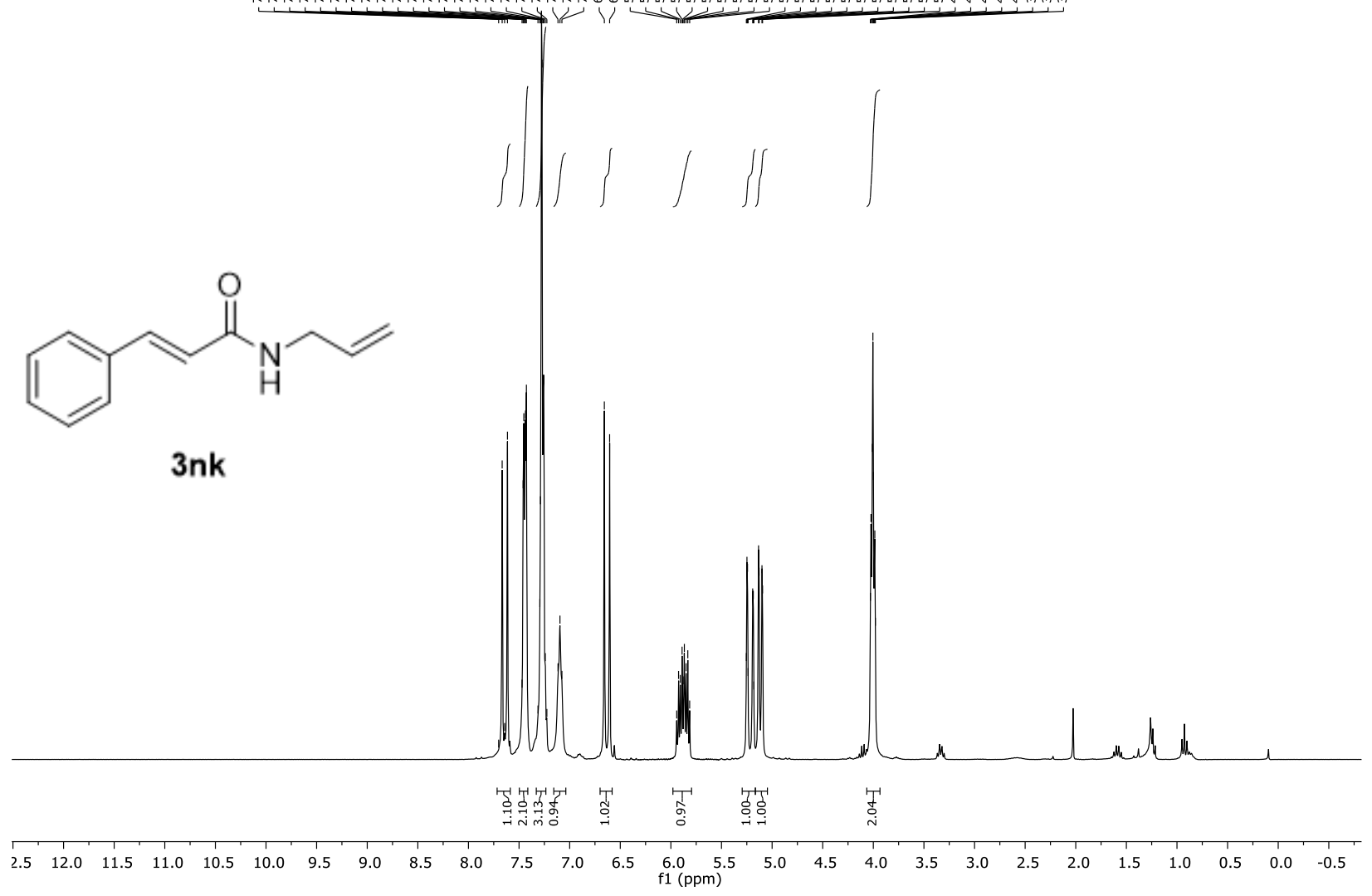

${ }^{1} \mathrm{H}$ NMR (300 MHz, Chloroform- $d$ ) N-allylcinnamamide (3nk) 

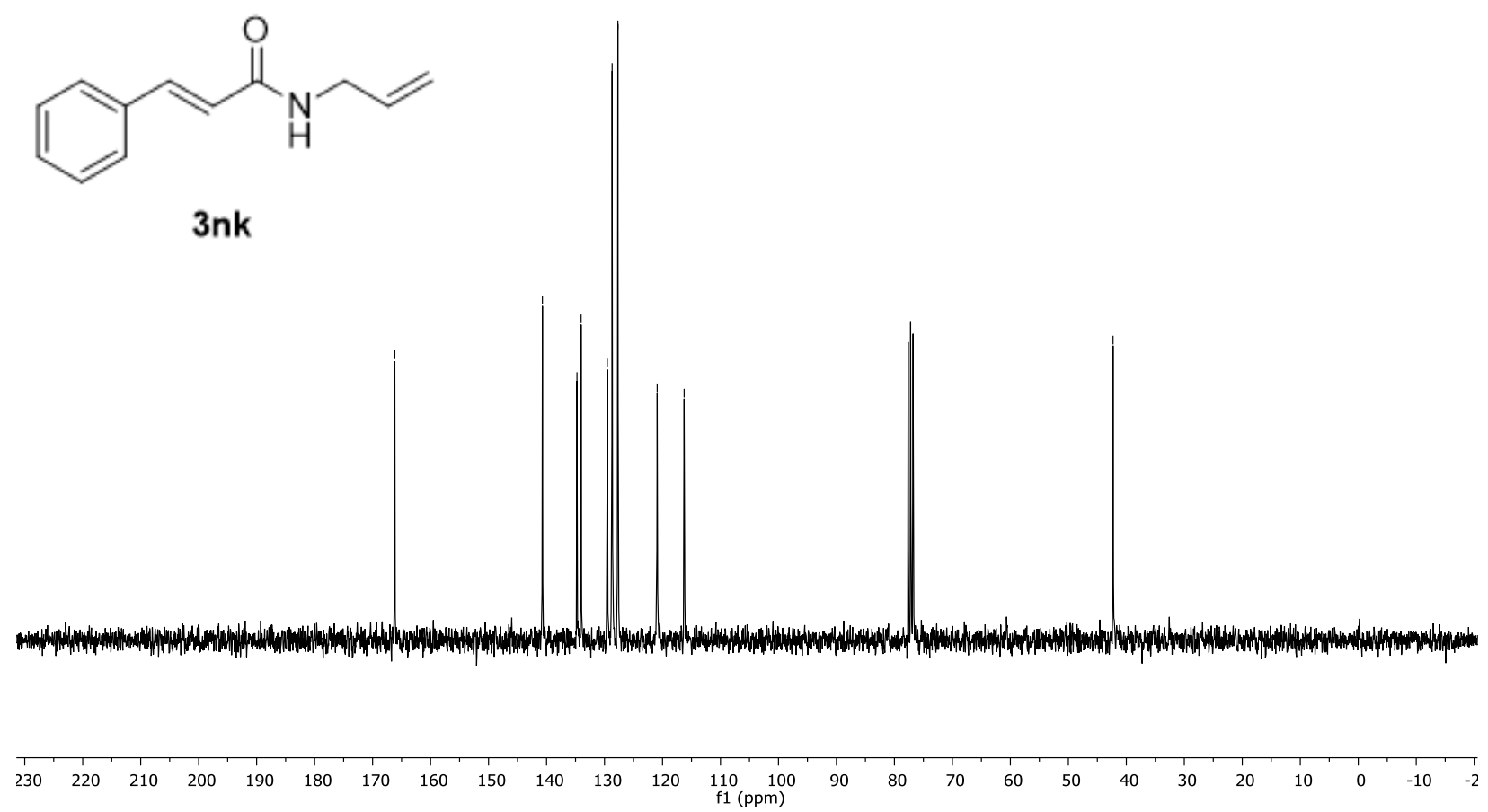

${ }^{13} \mathrm{C}$ NMR (75 MHz, Chloroform- $d$ ) N-allylcinnamamide (3nk)

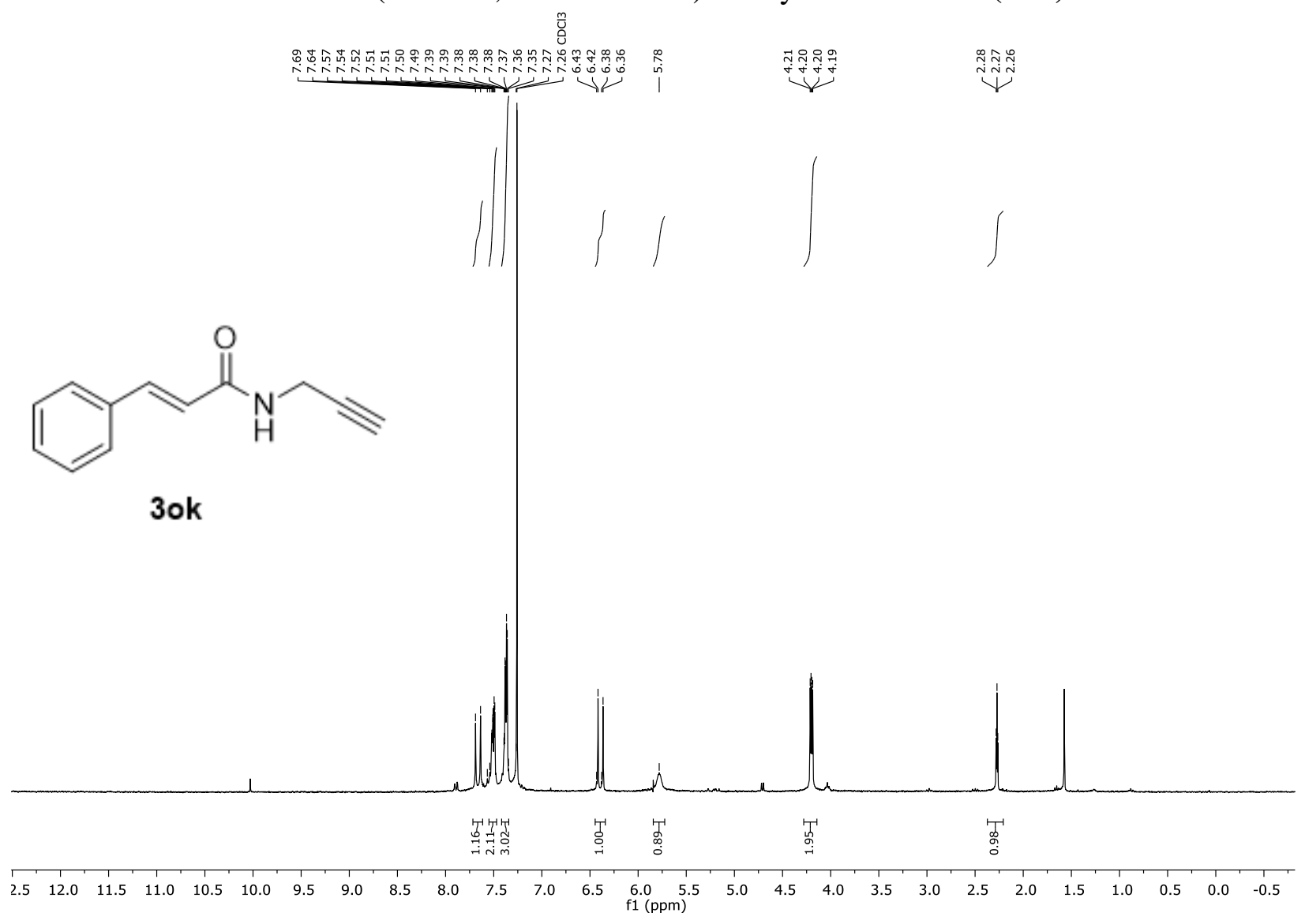

${ }^{1} \mathrm{H}$ NMR (300 MHz, Chloroform- $d$ ) N-(prop-2-yn-1-yl)cinnamamide (3ok) 


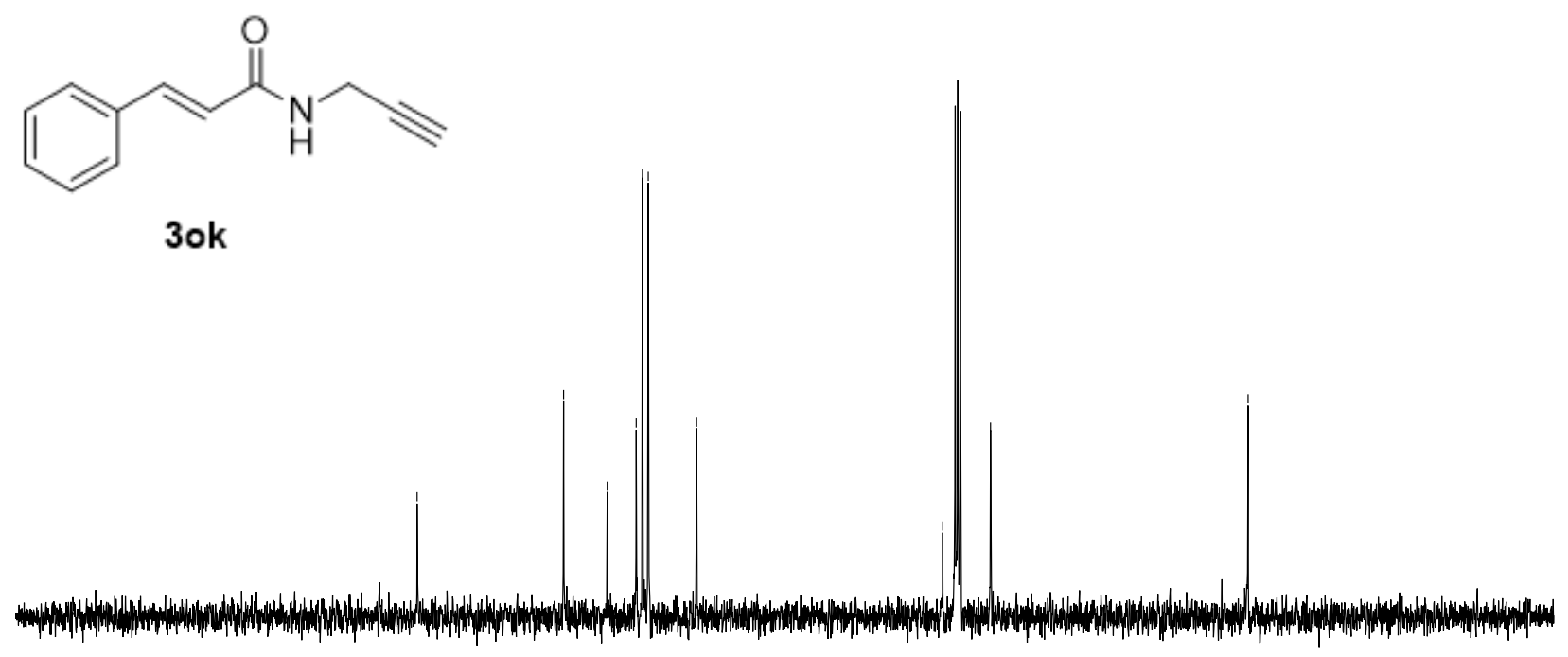

$\begin{array}{llllllllllllllllllllllllllllll}230 & 220 & 210 & 200 & 190 & 180 & 170 & 160 & 150 & 140 & 130 & 120 & \begin{array}{c}110 \\ f 1(\mathrm{ppm})\end{array} & 100 & 90 & 80 & 70 & 60 & 50 & 40 & 30 & 20 & 10 & 0 & -10 & -2\end{array}$

${ }^{13} \mathrm{C}$ NMR (75 MHz, Chloroform- $d$ ) N-(prop-2-yn-1-yl)cinnamamide (3ok)

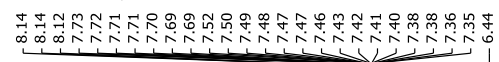

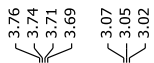

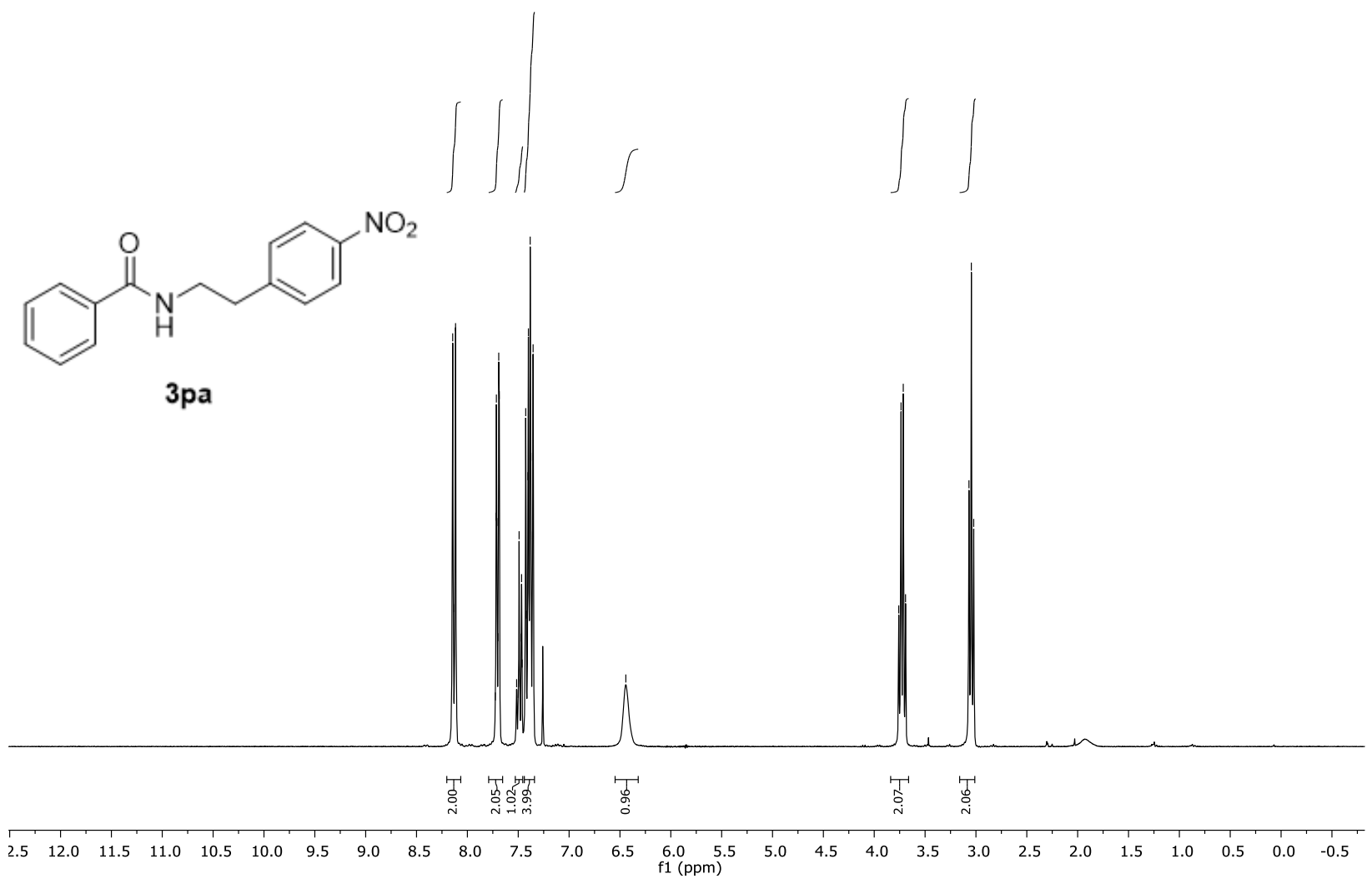

${ }^{1} \mathrm{H}$ NMR (300 MHz, Chloroform- $d$ ) N-(4-nitrophenethyl)benzamide (3pa) 


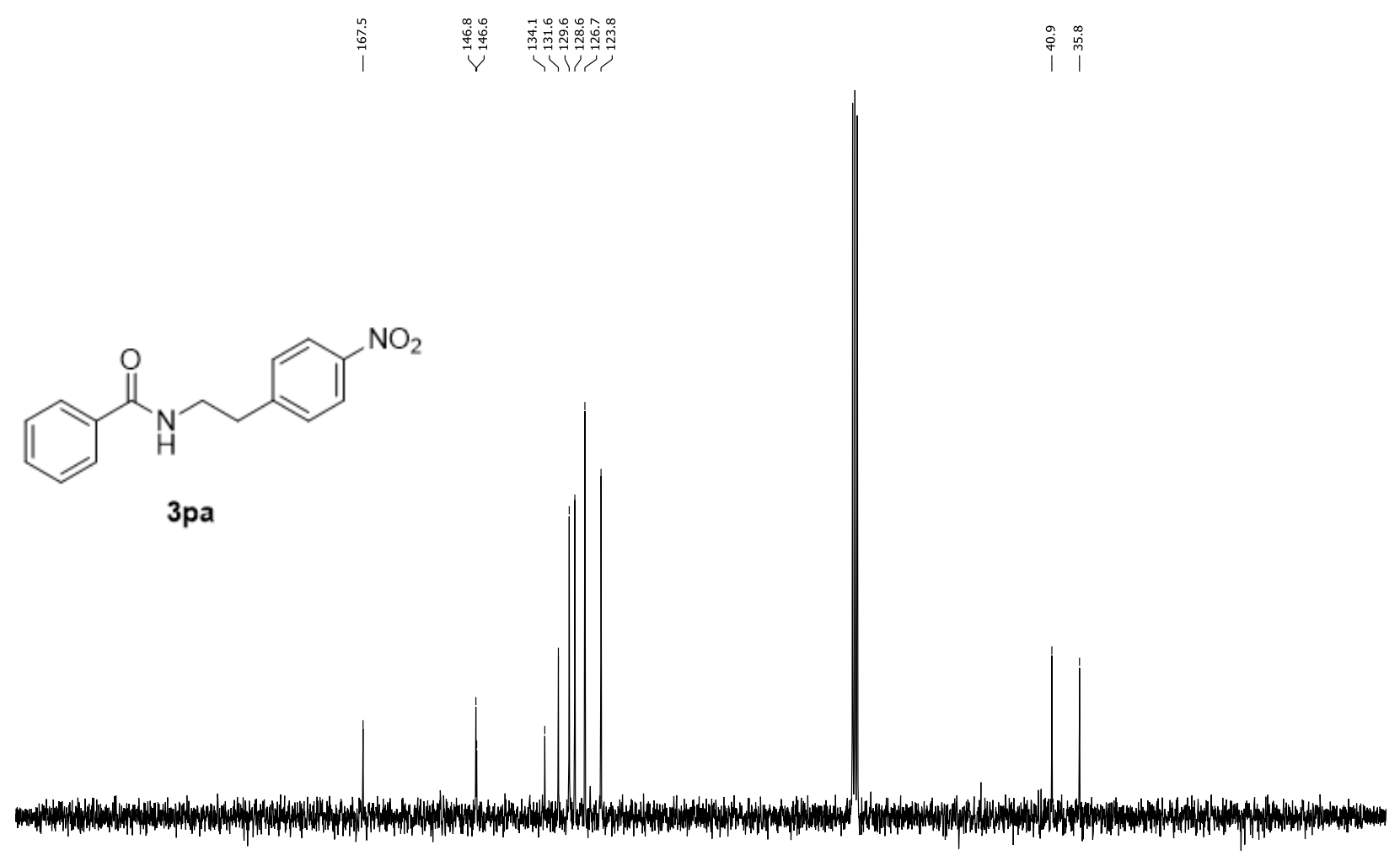

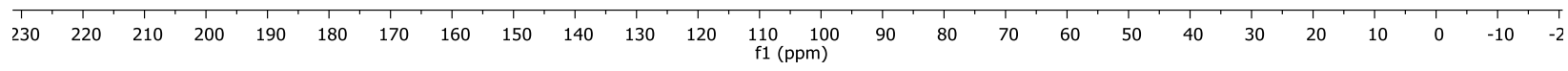

${ }^{13} \mathrm{C}$ NMR (75 MHz, Chloroform-d) N-(4-nitrophenethyl)benzamide (3pa)

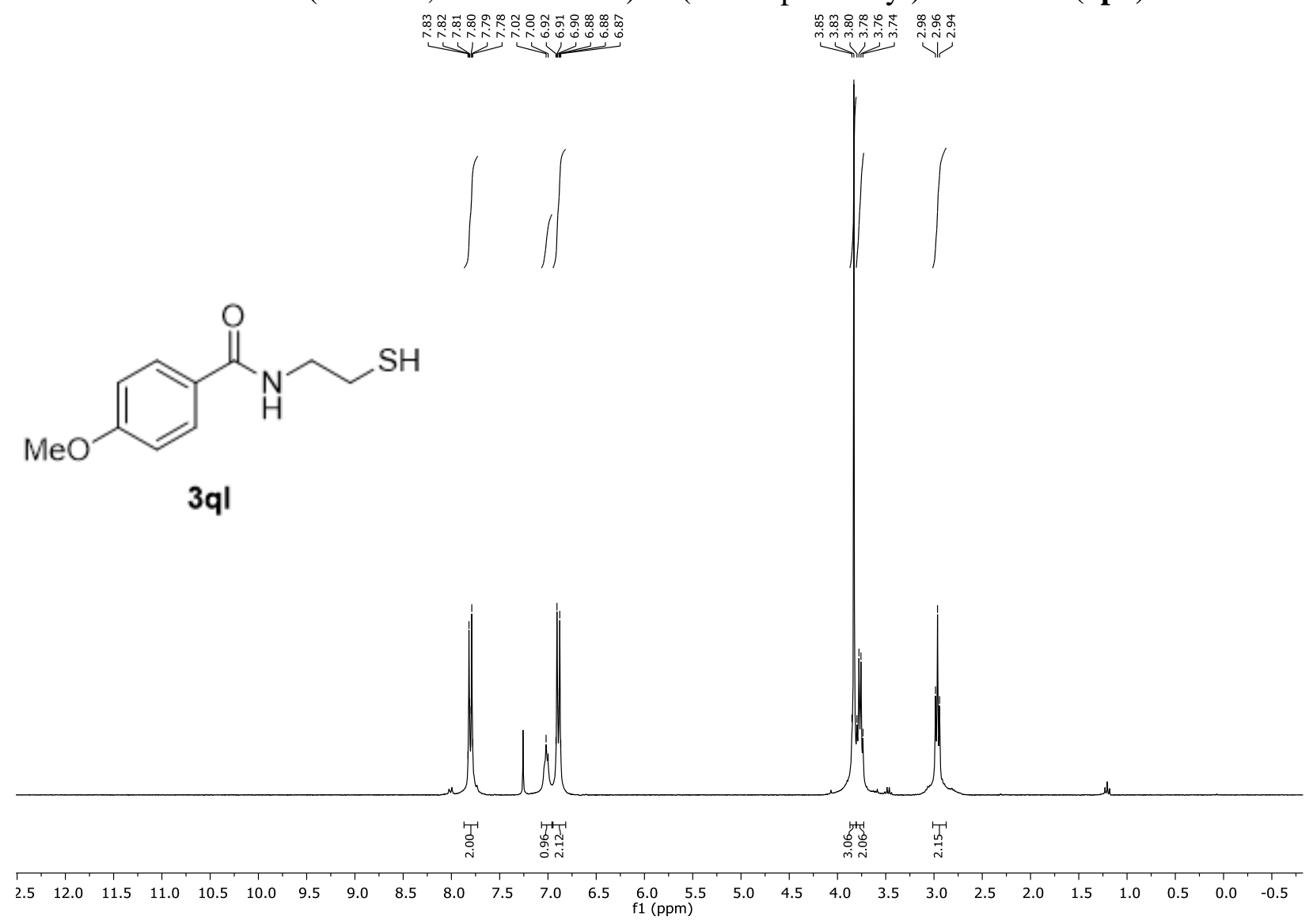

${ }^{1} \mathrm{H}$ NMR (300 MHz, Chloroform- $d$ ) N-(2-mercaptoethyl)-4-methoxybenzamide (3ql) 
<smiles>COc1ccc(C(=O)NCCS)cc1</smiles>

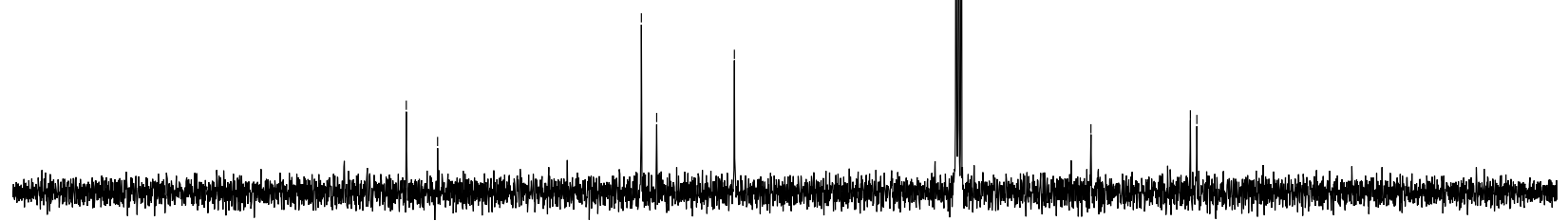

\begin{tabular}{lllllllllllllllllllllllllllllll}
\hline 230 & 220 & 210 & 200 & 190 & 180 & 170 & 160 & 150 & 140 & 130 & 120 & $\begin{array}{l}110 \\
\mathrm{f} 1(\mathrm{ppm})\end{array}$ & 90 & 80 & 70 & 60 & 50 & 40 & 30 & 20 & 10 & 0 & -10 & -2
\end{tabular}

${ }^{13} \mathrm{C}$ NMR (75 MHz, Chloroform- $d$ ) N-(2-mercaptoethyl)-4-methoxybenzamide (3ql) 年

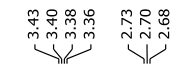

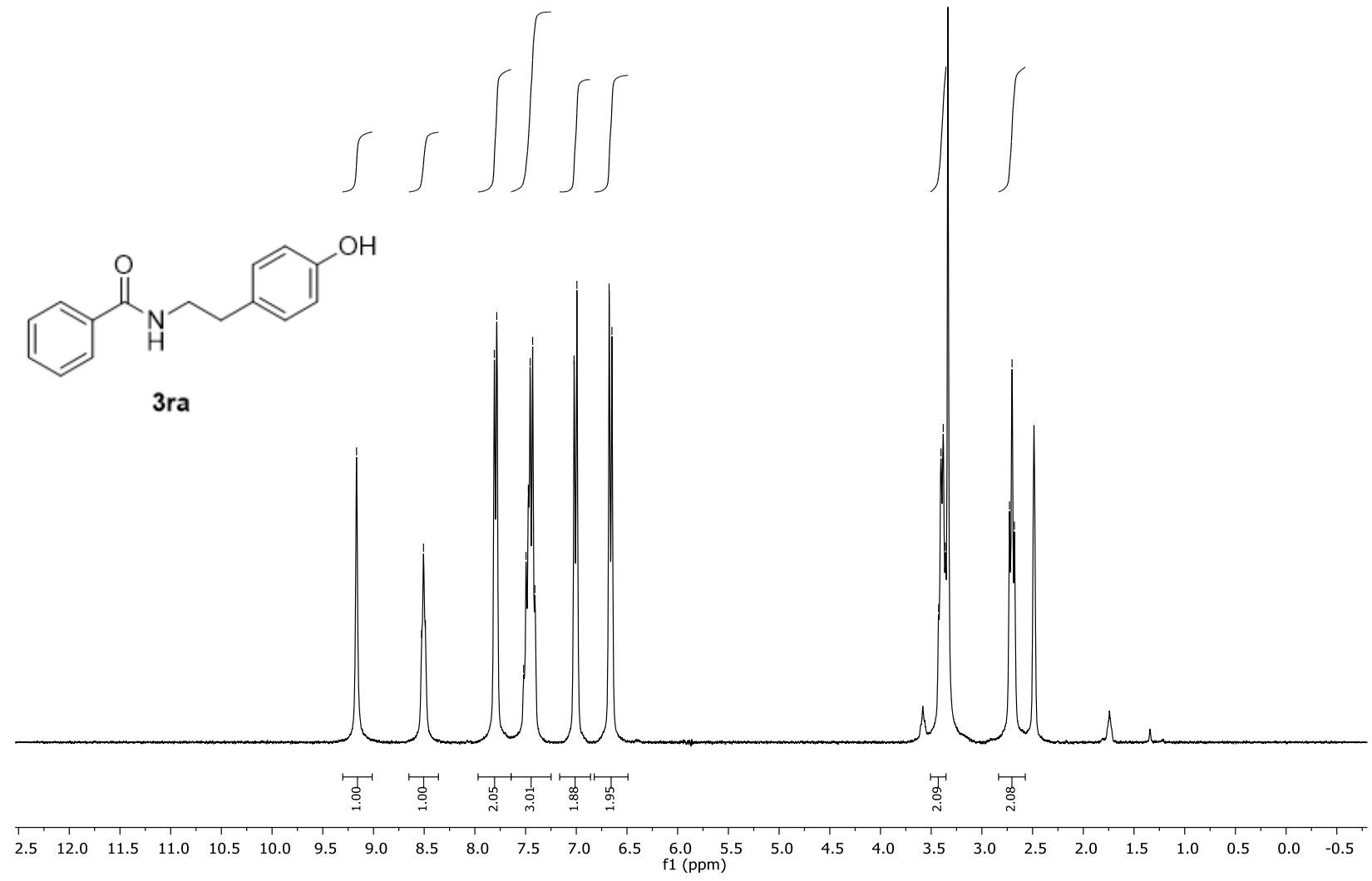

${ }^{1} \mathrm{H}$ NMR (300 MHz, DMSO-d6) N-(4-hydroxyphenethyl)benzamide (3ra) 


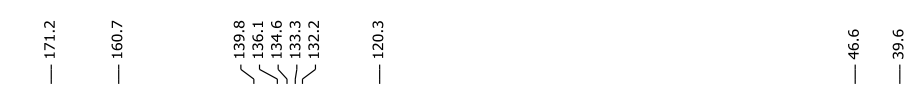<smiles>O=C(NCCc1ccc(O)cc1)c1ccccc1</smiles>

3ra

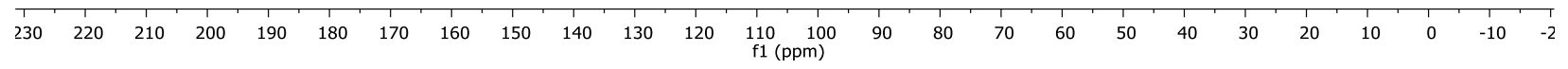

${ }^{13} \mathrm{C}$ NMR (75 MHz, DMSO-d6) N-(4-hydroxyphenethyl)benzamide (3ra)

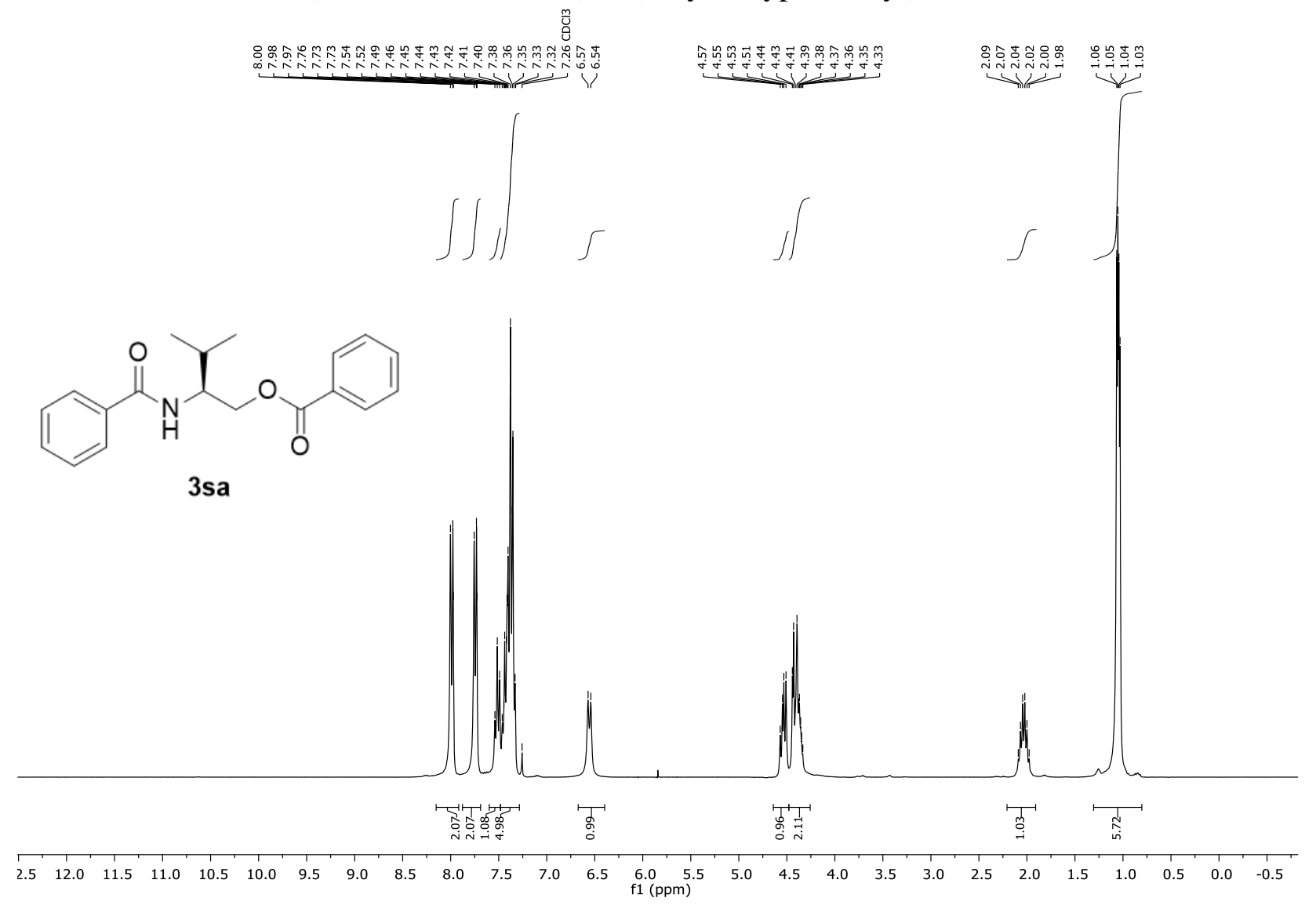

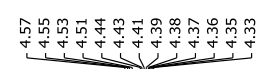

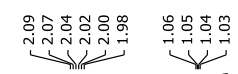

${ }^{1} \mathrm{H}$ NMR (300 MHz, Chloroform- $d$ ) (S)-2-benzamido-3-methylbutyl benzoate (3sa) 

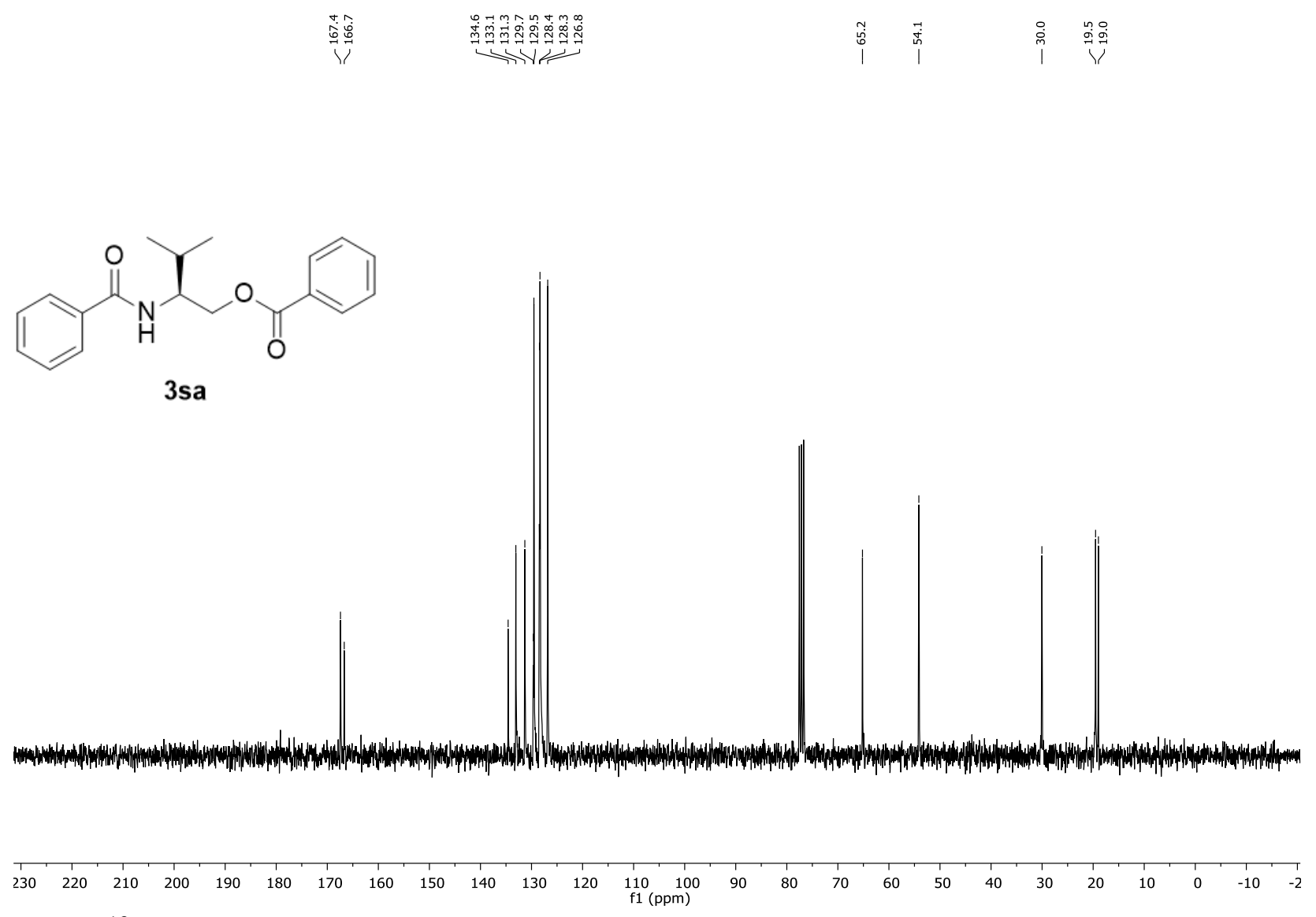

${ }^{13} \mathrm{C}$ NMR (75 MHz, Chloroform-d) (S)-2-benzamido-3-methylbutyl benzoate (3sa)

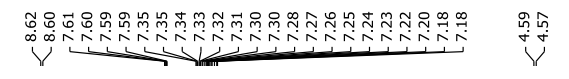

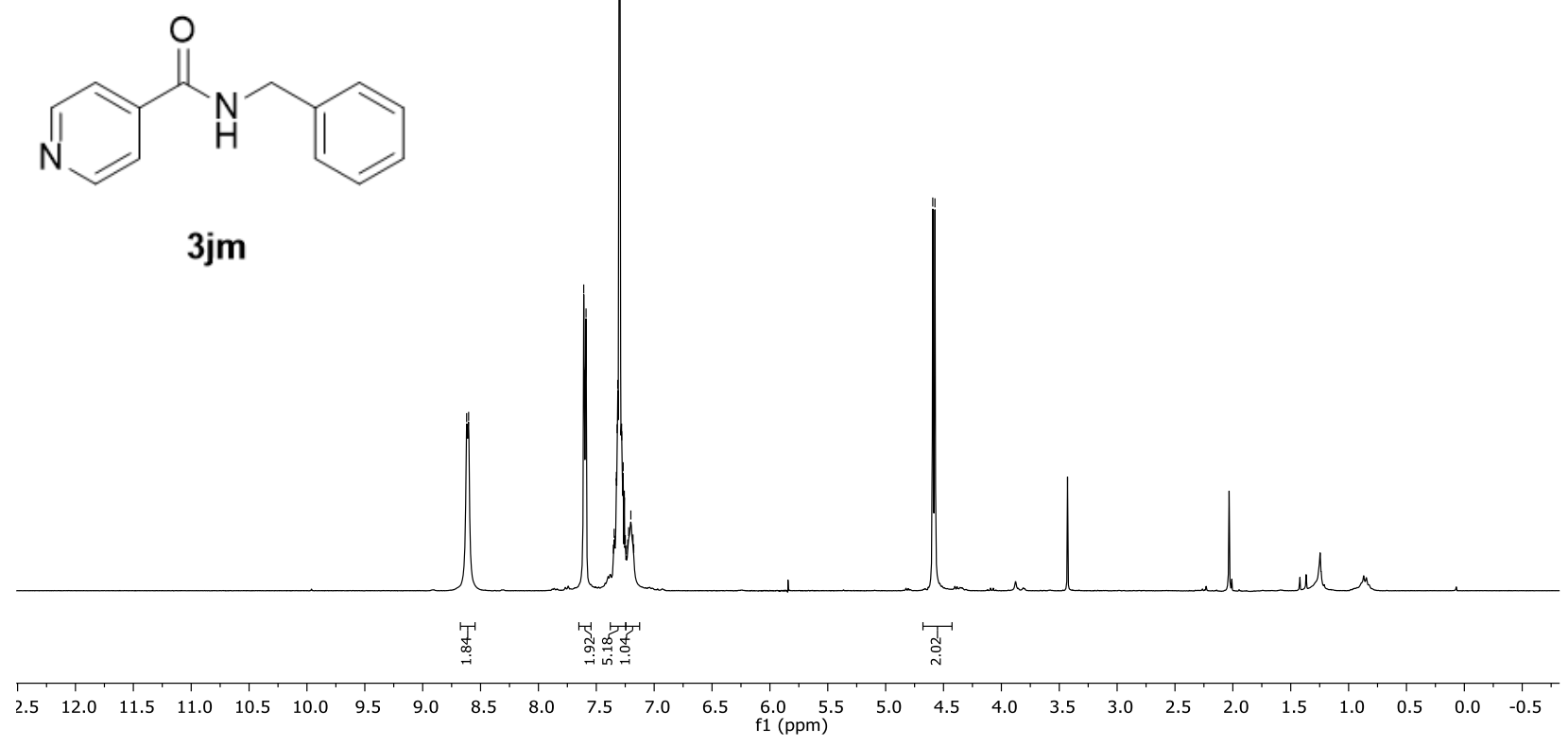

${ }^{1} \mathrm{H}$ NMR (300 MHz, Chloroform- $d$ ) N-benzylisonicotinamide (3jm) 
<smiles>O=C(NCc1ccccc1)c1ccncc1</smiles>

$3 \mathrm{jm}$
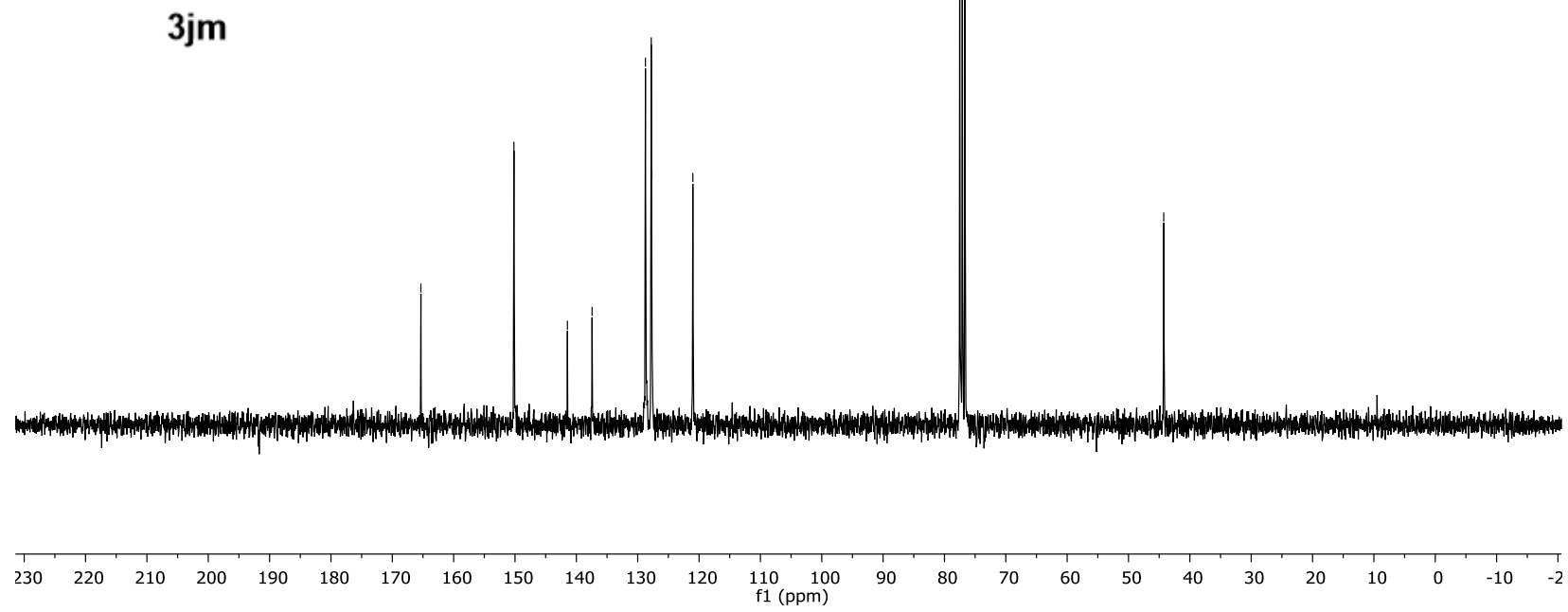

${ }^{13} \mathrm{C}$ NMR (75 MHz, Chloroform- $d$ ) N-benzylisonicotinamide (3jm)
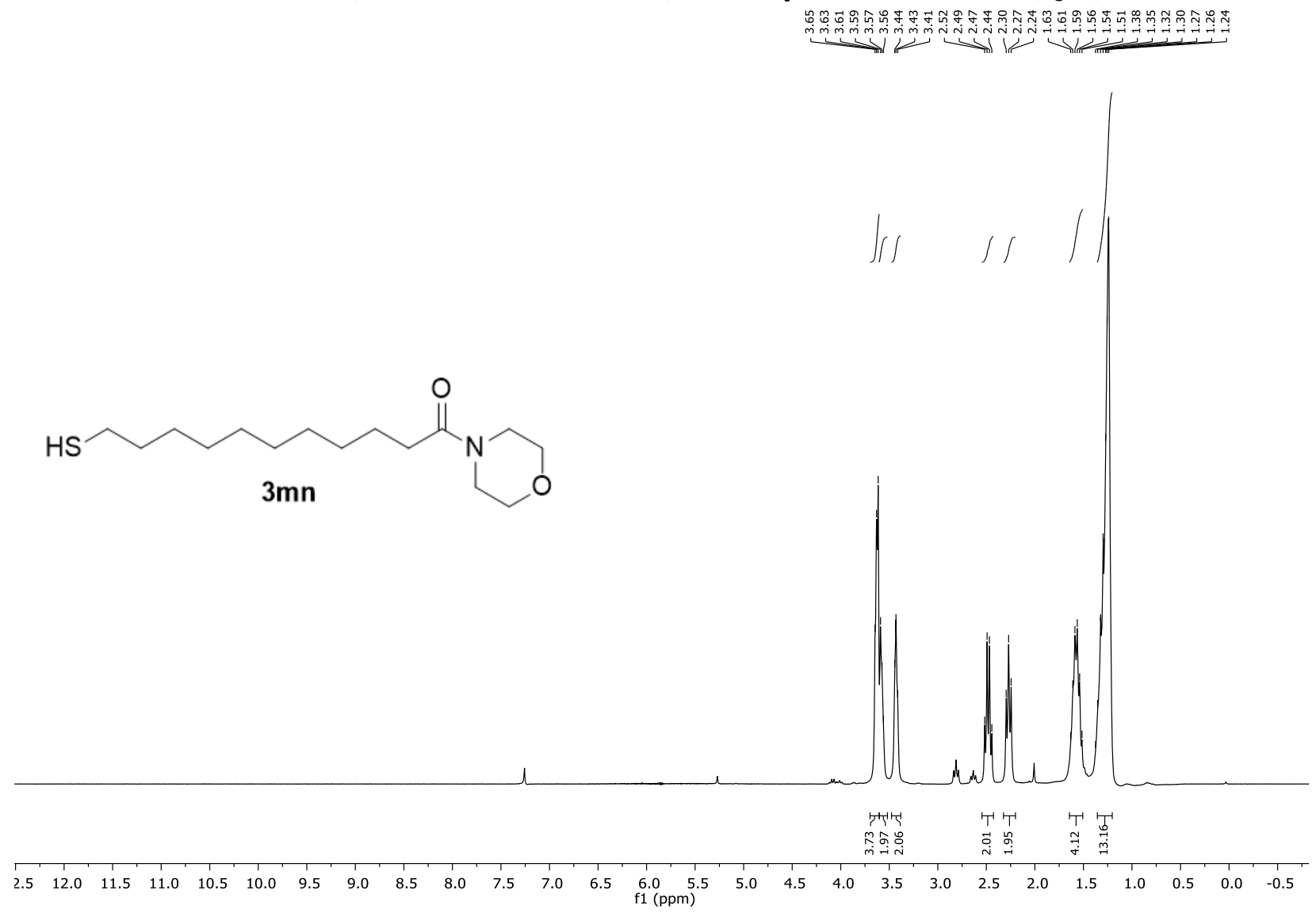

${ }^{1} \mathrm{H}$ NMR (300 MHz, Chloroform- $d$ ) 11-mercapto-1-morpholinoundecan-1-one (3mn) 

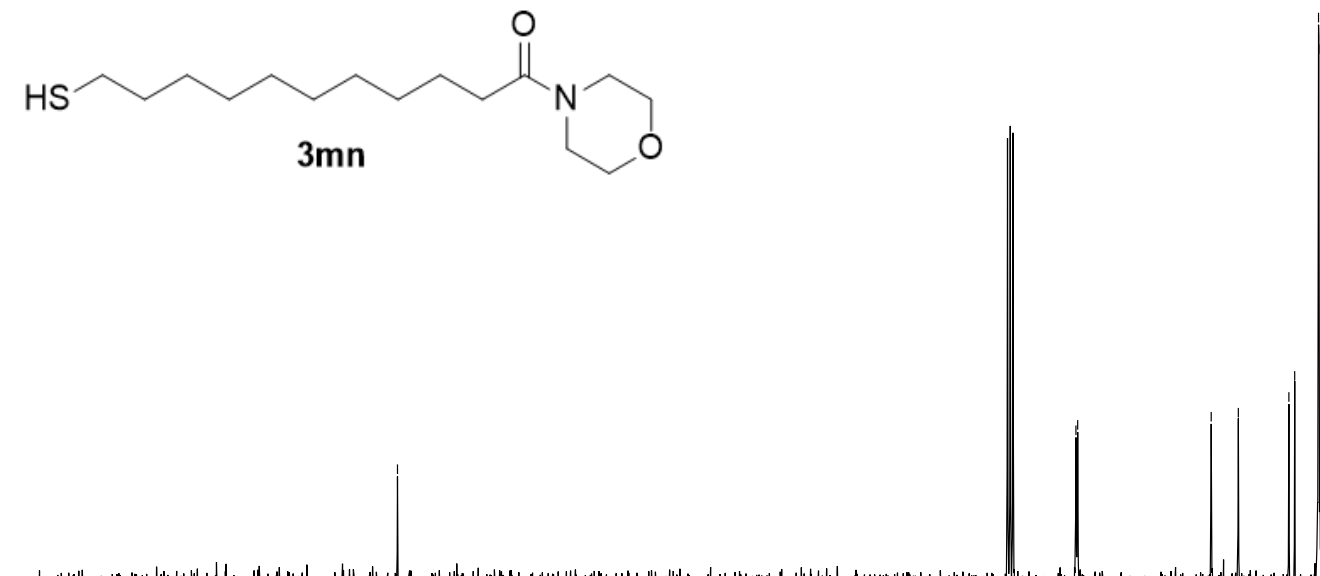

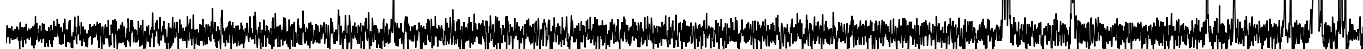
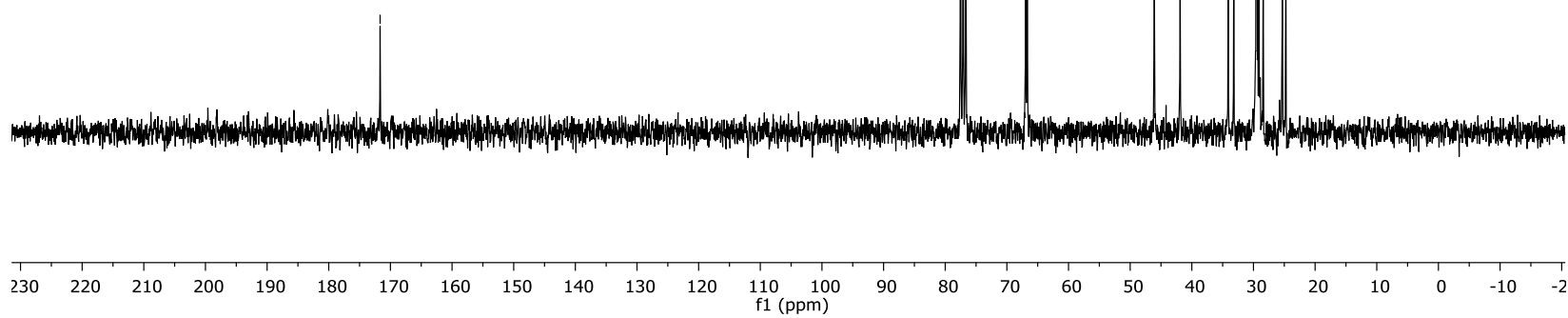

${ }^{13} \mathrm{C}$ NMR (75 MHz, Chloroform- $d$ ) 11-mercapto-1-morpholinoundecan-1-one (3mn)

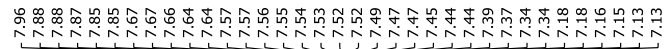

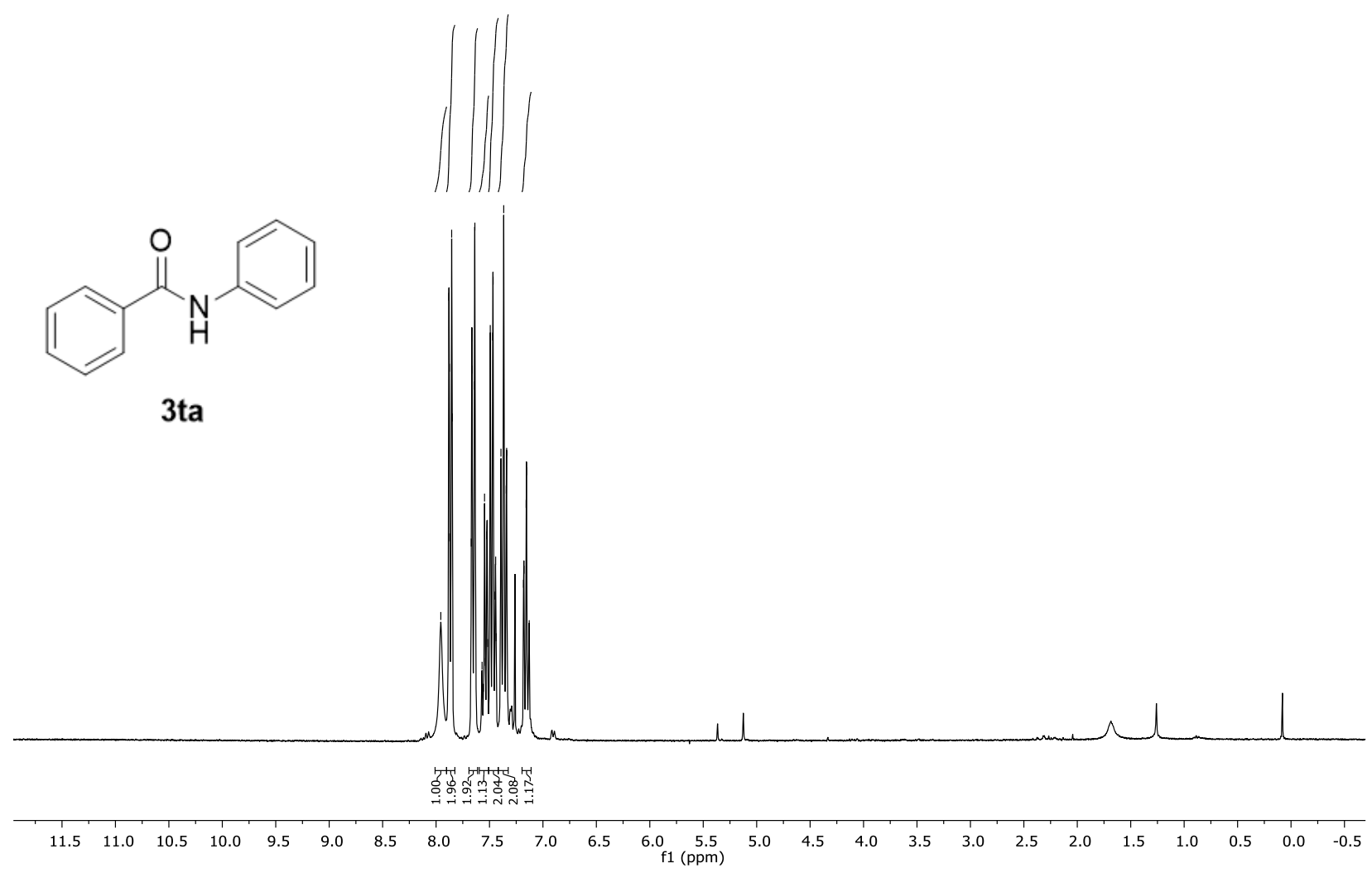

${ }^{1} \mathrm{H}$ NMR (300 MHz, Chloroform- $d$ ) N-phenylbenzamide (3ta) 
<smiles>O=C(Nc1ccccc1)c1ccccc1</smiles>

3ta

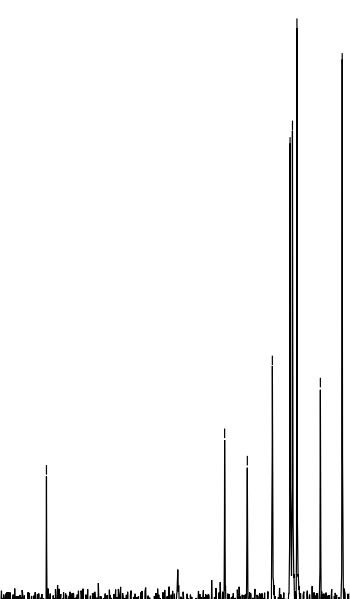

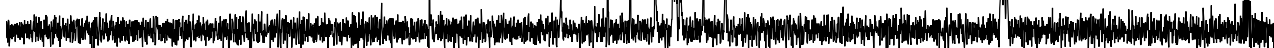
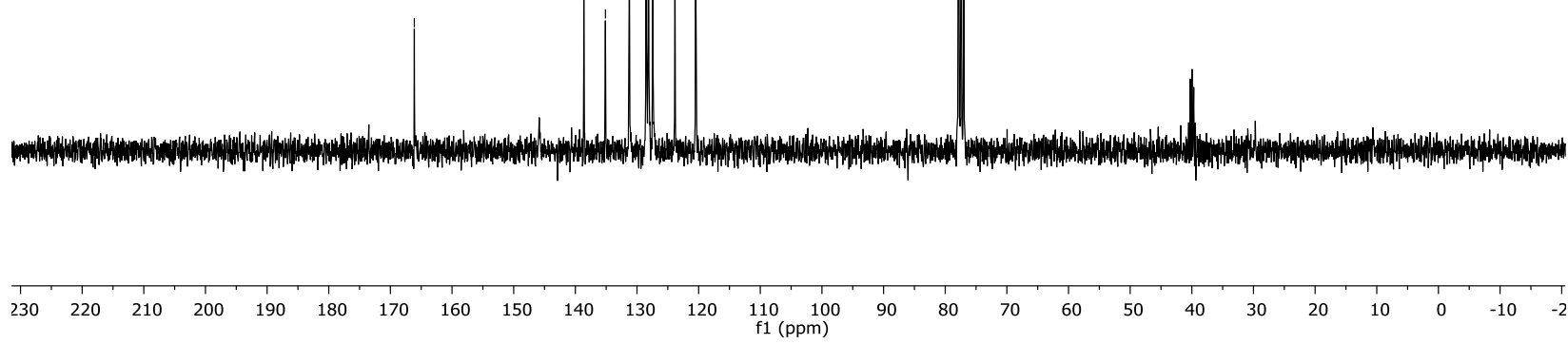

${ }^{13} \mathrm{C}$ NMR (75 MHz, Chloroform- $d$ ) N-phenylbenzamide (3ta)
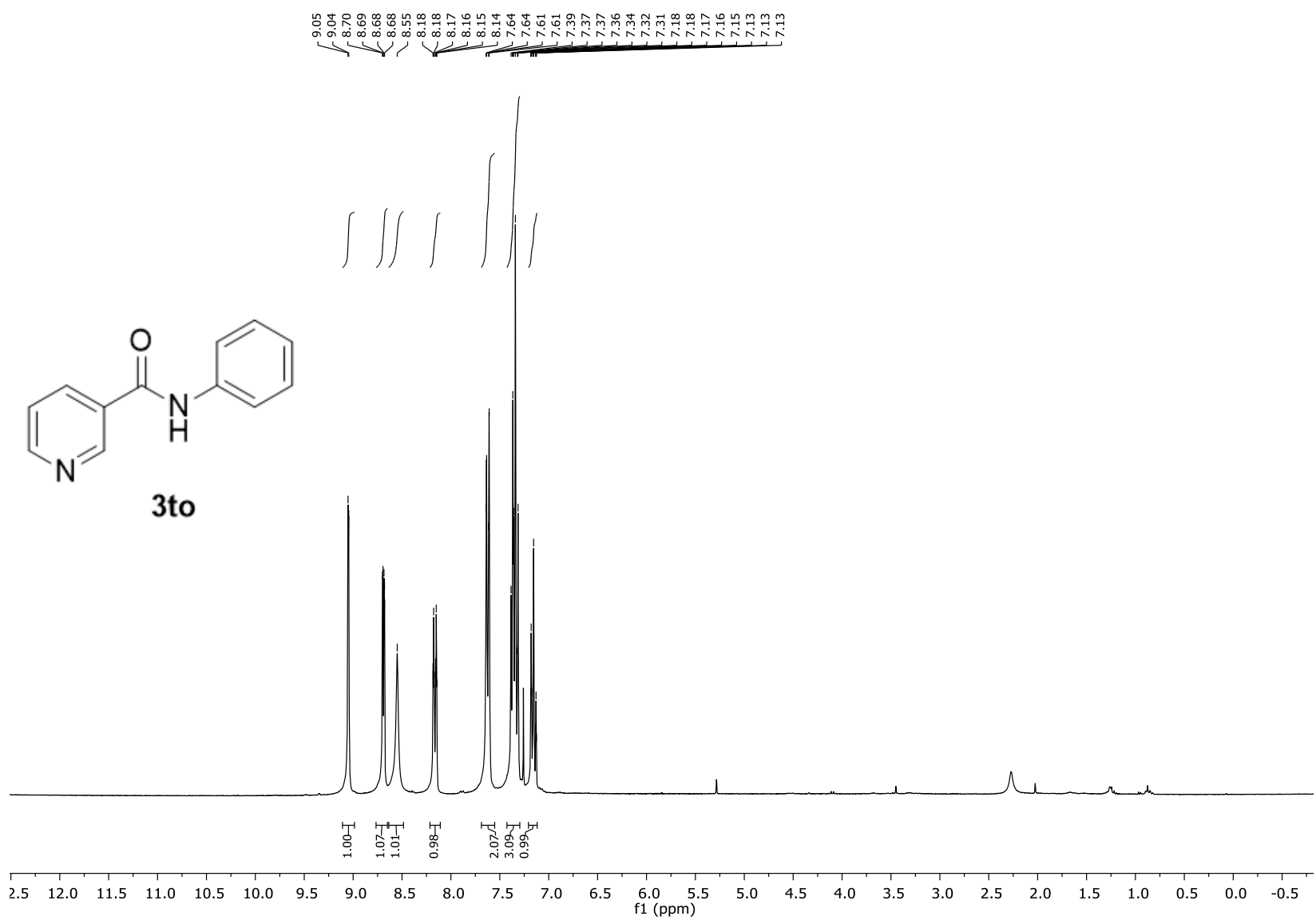

${ }^{1} \mathrm{H}$ NMR (300 MHz, Chloroform- $d$ ) N-phenylnicotinamide (3to) 
<smiles>O=C(Nc1ccccc1)c1cccnc1</smiles>
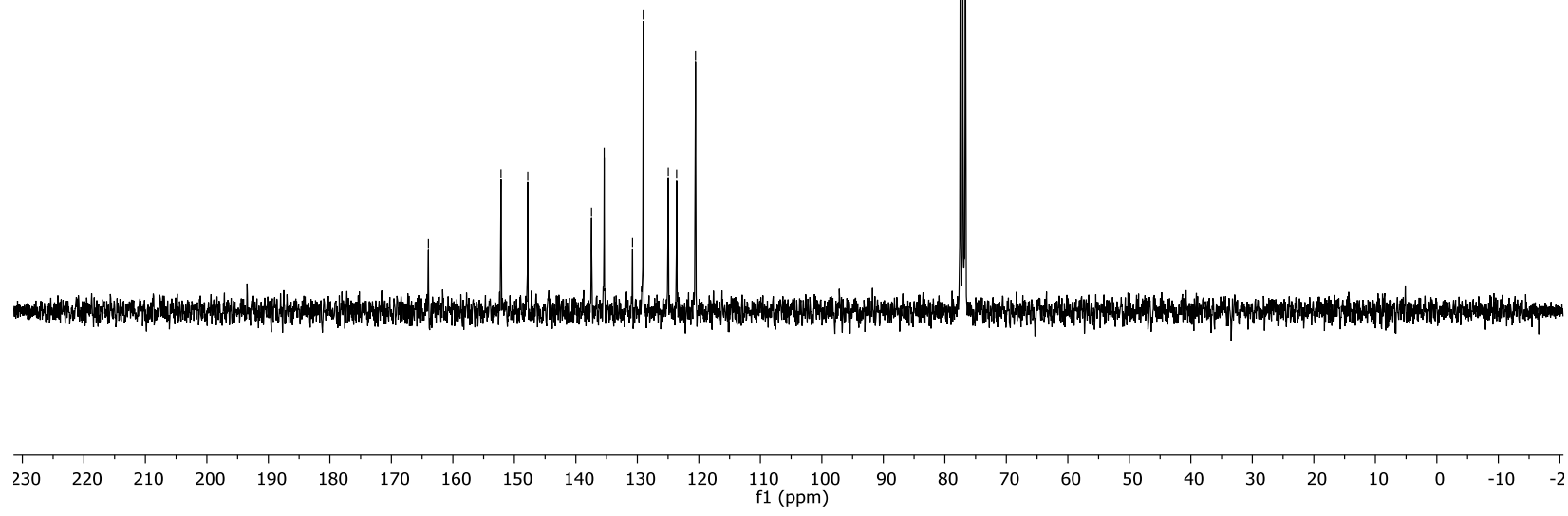

${ }^{13} \mathrm{C}$ NMR (75 MHz, Chloroform- $d$ ) N-phenylnicotinamide (3to)

Spectra from mechanistic studies:

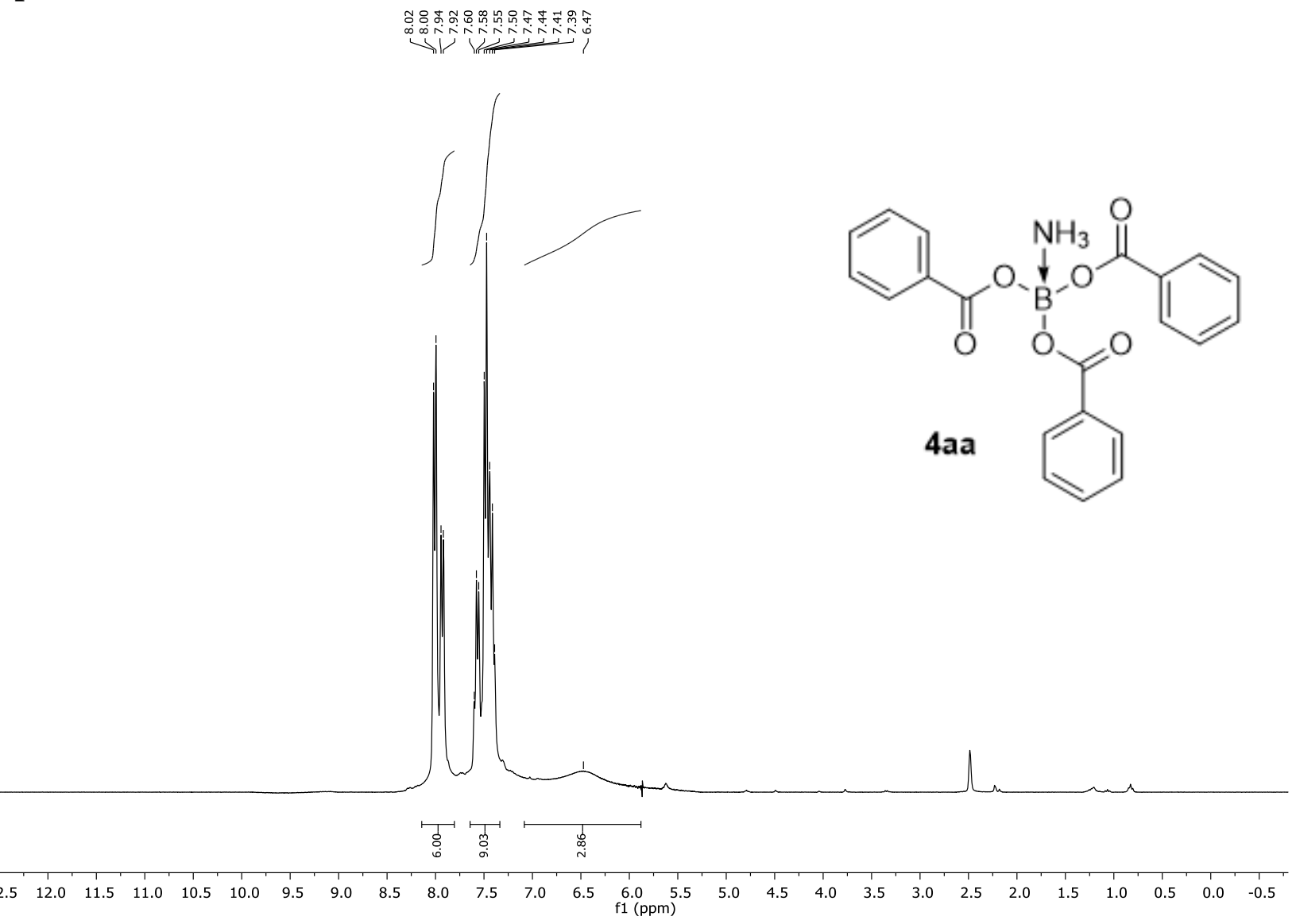

${ }^{1} \mathrm{H}$ NMR (300 MHz, DMSO-d6) Ammonia-tribenzyloxyborane (4aa) 


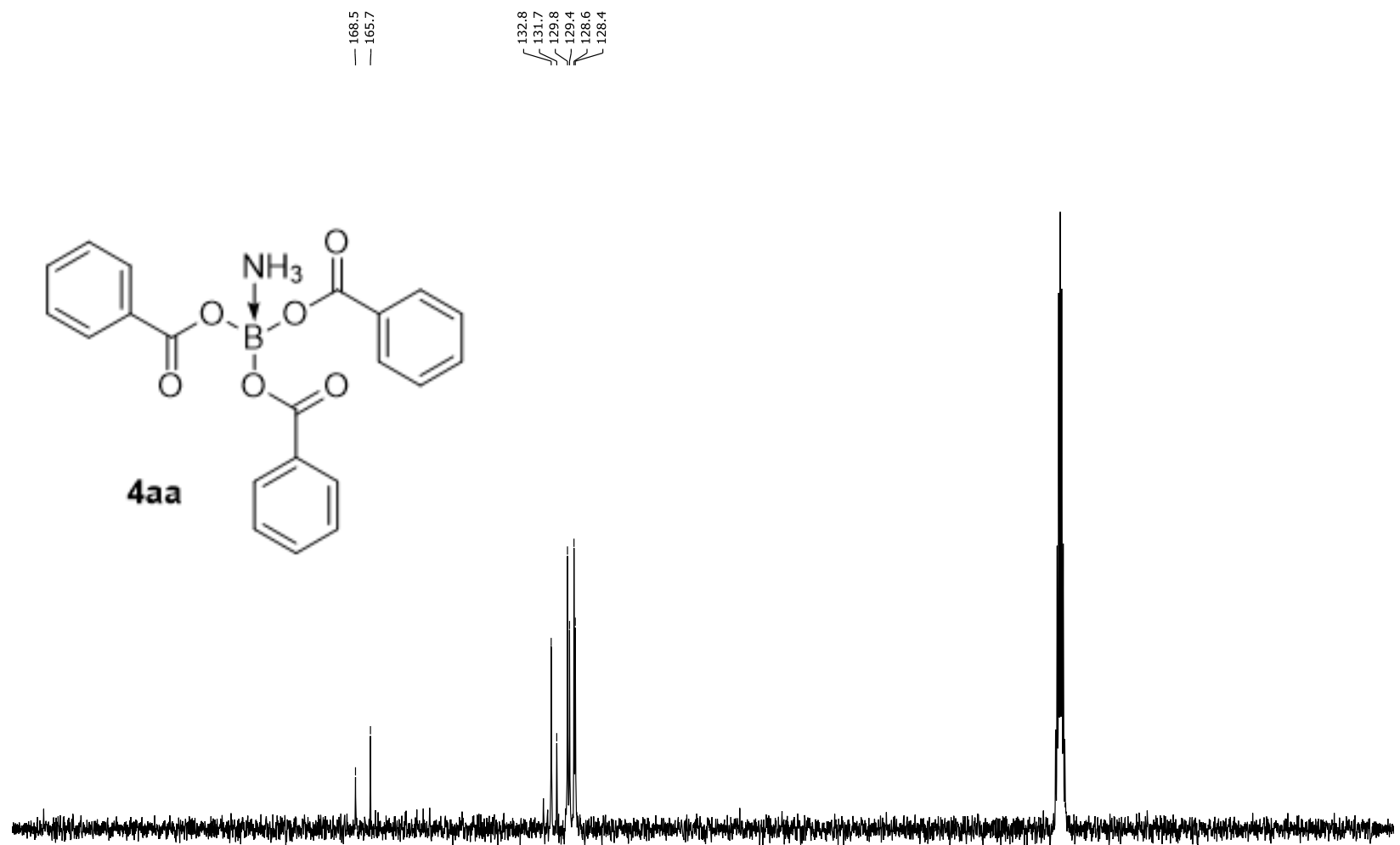

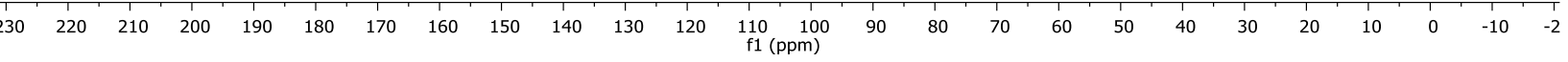

${ }^{13} \mathrm{C}$ NMR (75 MHz, DMSO-d6) Ammonia-tribenzyloxyborane (4aa)

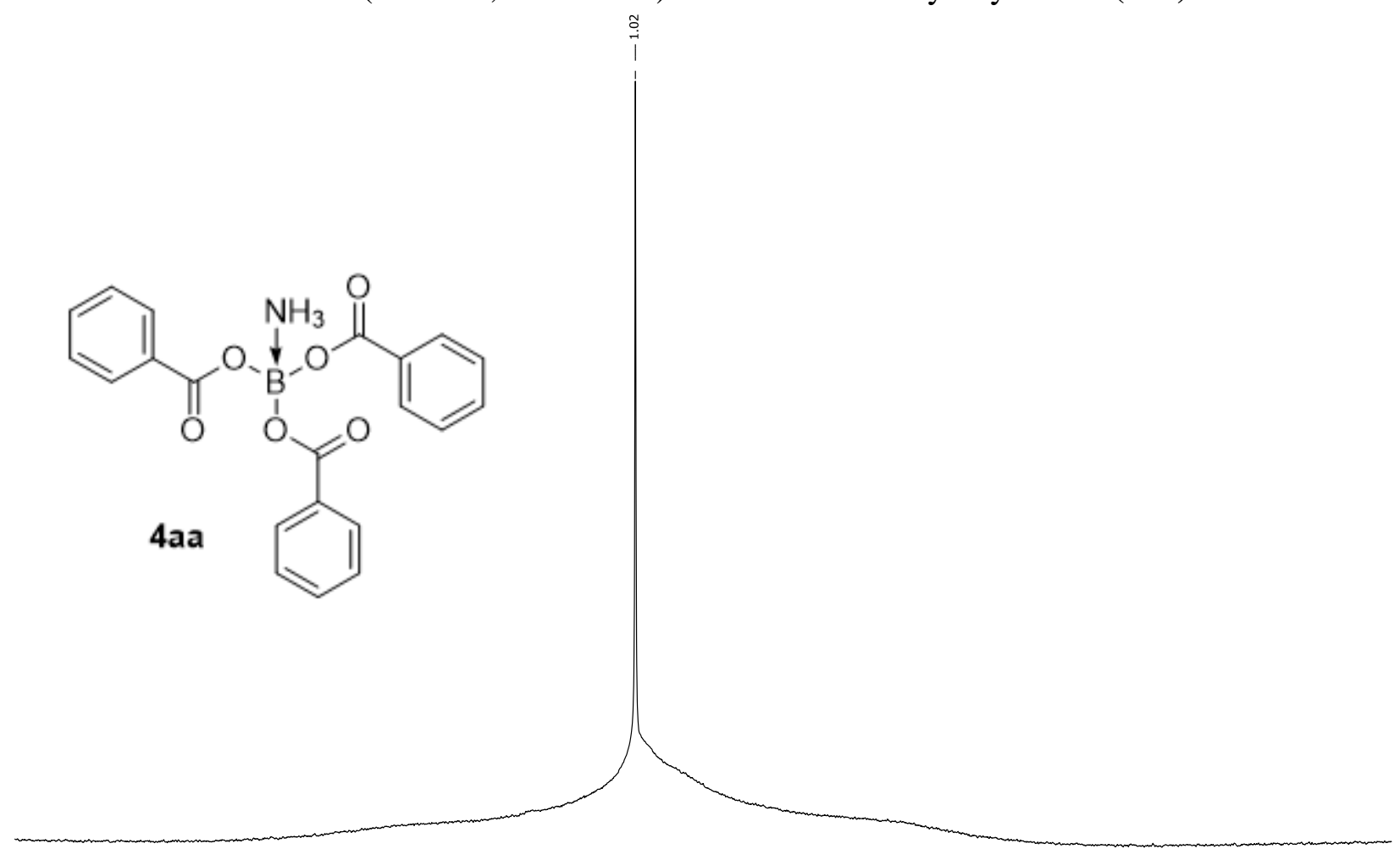

$100 \quad 90 \quad 80$

${ }^{11} \mathrm{~B}$ NMR (96 MHz, DMSO-d6) Ammonia-tribenzyloxyborane (4aa) 


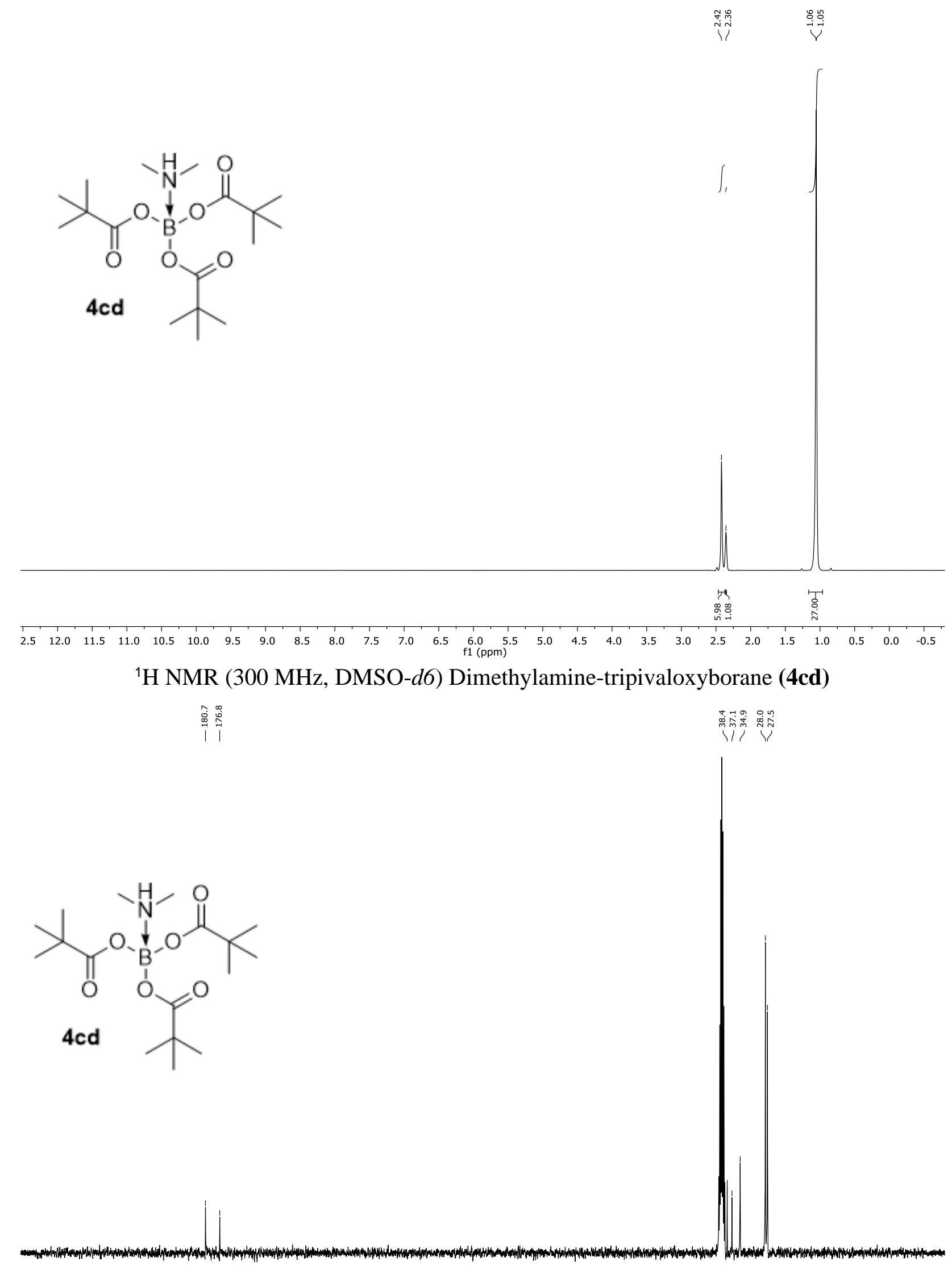

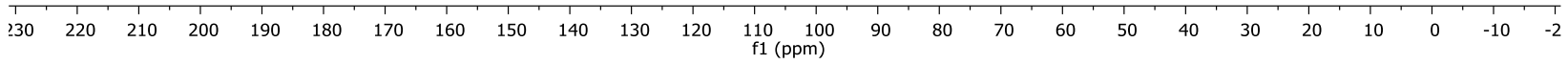

${ }^{13} \mathrm{C}$ NMR (75 MHz, DMSO-d6) Dimethylamine-tripivaloxyborane (4cd) 

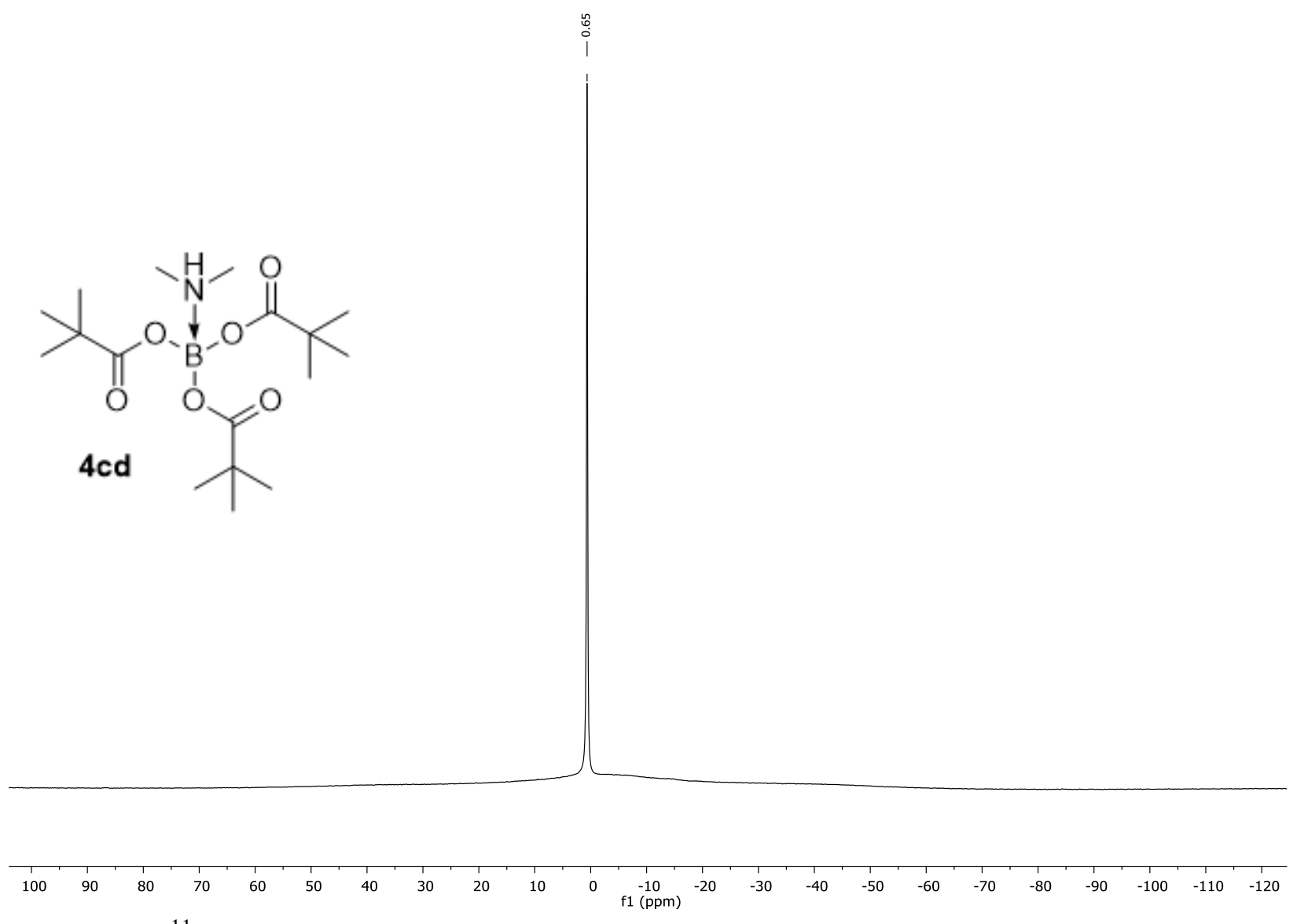

${ }^{11}$ B NMR (96 MHz, DMSO-d6) Dimethylamine-tripivaloxyborane (4cd)

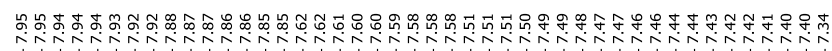

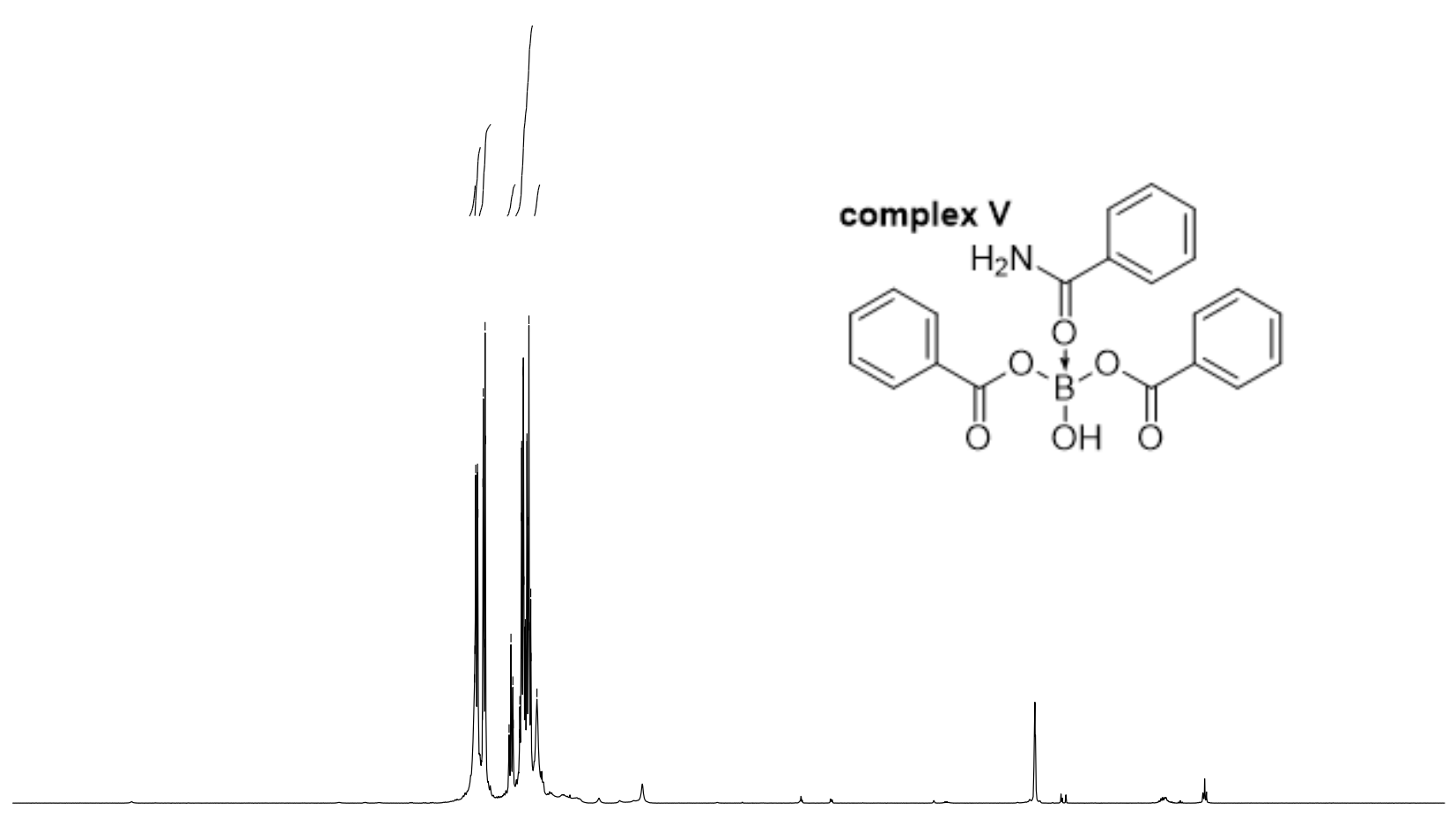

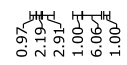

$\begin{array}{lllllllllllllllllllllllllllllllllll}12.0 & 11.5 & 11.0 & 10.5 & 10.0 & 9.5 & 9.0 & 8.5 & 8.0 & 7.5 & 7.0 & 6.5 & 6.0 & 5.5 & 5.0 & 4.5 & 4.0 & 3.5 & 3.0 & 2.5 & 2.0 & 1.5 & 1.0 & 0.5 & 0.0 & -0.5 & -1.0 & -1\end{array}$

${ }^{1} \mathrm{H}$ NMR (300 MHz, DMSO-d6) Benzamide-dibenzyloxyboric anhydride (complex V) 
<smiles>NC(=O)c1ccccc1</smiles>
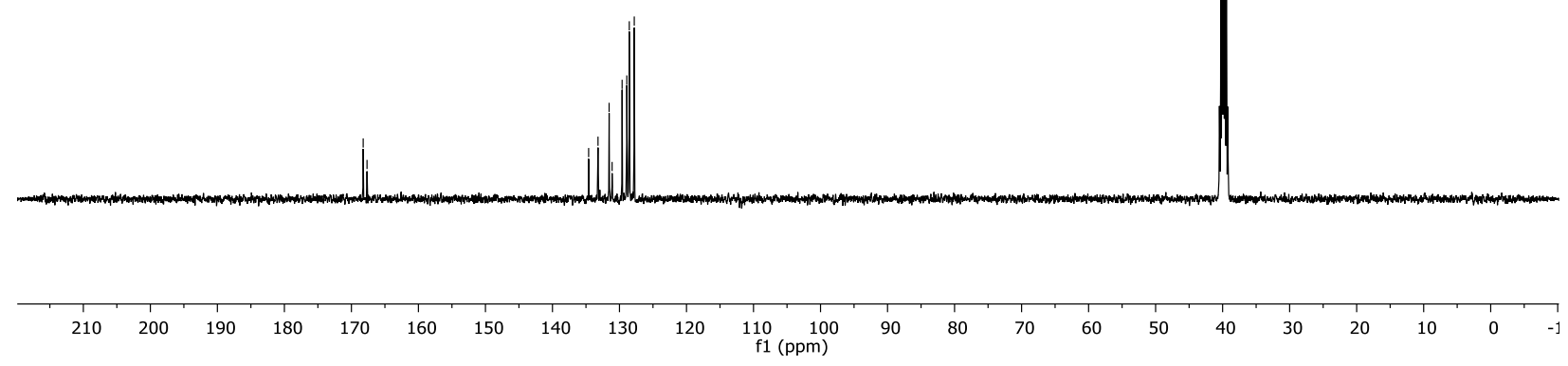

${ }^{13} \mathrm{C}$ NMR (75 MHz, DMSO-d6) Benzamide-dibenzyloxyboric anhydride (complex V)

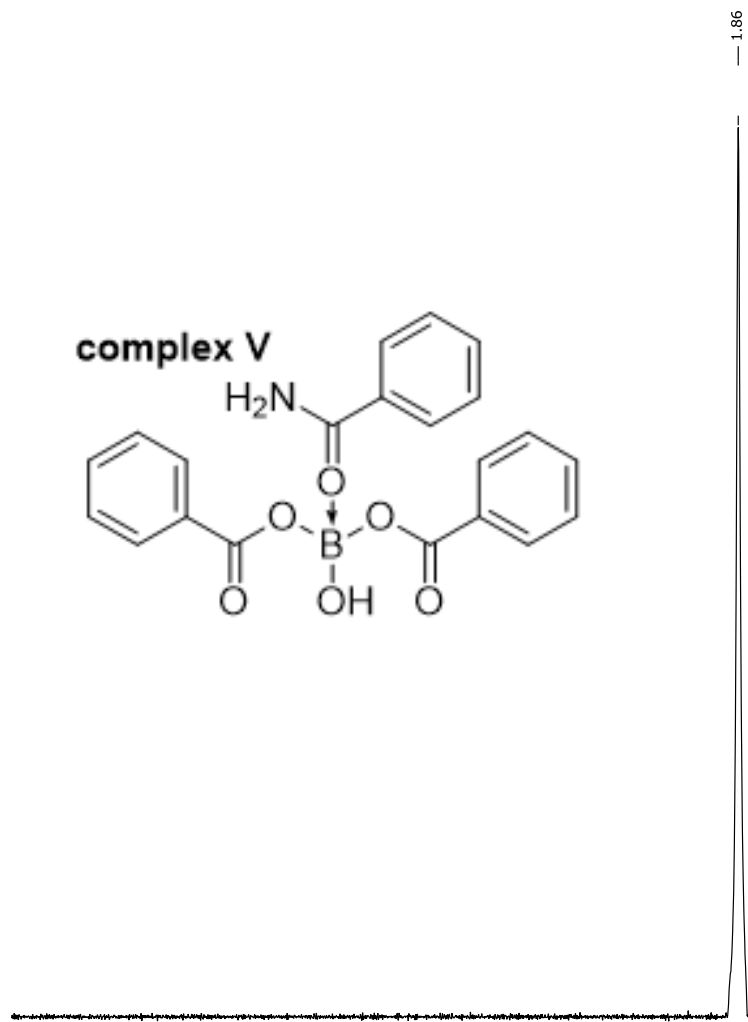

${ }^{11}$ B NMR (96 MHz, DMSO- $d 6$ ) Benzamide-dibenzyloxyboric anhydride (complex V) 